\title{
The association of smoking status with SARS-CoV-2 infection, hospitalisation and mortality from COVID-19: A living rapid evidence review with Bayesian meta-analyses (version 12)
}

\author{
David Simons ${ }^{1}$, Lion Shahab², Jamie Brown², Olga Perski \\ 1 Royal Veterinary College, RVC \\ 2 University College London, University of London \\ Funding: The author(s) received no specific funding for this work. \\ Potential competing interests: The author(s) declared that no potential competing interests exist.
}

\section{Abstract}

Aims: To estimate the association of smoking status with rates of i) infection, ii) hospitalisation, iii) disease severity in hospitalised patients, and iv) mortality from SARS-CoV-2/COVID-19 disease. Design: Living rapid review of observational and experimental studies with random-effects hierarchical Bayesian meta-analyses. Published articles and pre-prints were identified via MEDLINE and medRxiv

Setting: Community or hospital. No restrictions on location

Participants: Adults who received a SARS-CoV-2 test or a COVID-19 diagnosis.

Measurements: Outcomes were SARS-CoV-2 infection, hospitalisation, disease severity and mortality stratified by smoking status. Study quality was assessed (i.e. 'good,' 'fair' and 'poor').

Findings: v12 (searches up to 2021-07-18) included 547 studies with 87 'good' and 'fair' quality studies included in unadjusted meta-analyses. 171 studies (31.3\%) reported current, former and never smoking status with the remainder using broader categories. Recorded smoking prevalence among people with COVID-19 was generally lower than national prevalence. Current compared with never smokers were at reduced risk of SARS-CoV-2 infection $(\mathrm{RR}=0.67,95 \%$ Credible Interval $(\mathrm{Crl})=0.60-0.75, \mathrm{~T}=0.27)$. Data for former smokers were inconclusive $(\mathrm{RR}=0.99,95 \% \mathrm{Crl}=$ $0.94-1.05, T=0.12)$ but favoured there being no important association $(<1 \%$ probability of $R R \geq 1.1)$. Former compared with never smokers were at increased risk of hospitalisation ( $R R=1.27$, Crl $=1.15-1.40, \mathrm{~T}=0.20)$, greater disease severity $(\mathrm{RR}=1.69, \mathrm{Crl}=1.30-2.22, \mathrm{~T}=0.43)$ and mortality $(\mathrm{RR}=1.59, \mathrm{Crl}=1.34-1.89, \mathrm{~T}=0.37)$. Current compared with never smokers were at increased risk of greater disease severity ( $R R 1.3,95 \% \mathrm{Crl}=1.01-1.71, \mathrm{~T}=0.32)$. Data for current smokers on hospitalisation and mortality were inconclusive $(\mathrm{RR}=1.10,95 \% \mathrm{Crl}=0.97-1.24, \mathrm{~T}=0.23 ; \mathrm{RR}$ $=1.13,95 \% \mathrm{Crl}=0.90-1.40, \mathrm{~T}=0.41$, respectively) but favoured there being no important associations $(50 \%$ and $60 \%$ probability of $\mathrm{RR} \geq 1.1$, respectively).

Conclusions: Compared with never smokers, current smokers appear to be at reduced risk of SARS-CoV-2 infection and increased risk of greater in-hospital disease severity, while former smokers appear to be at increased risk of hospitalisation, greater in-hospital disease severity and mortality from COVID-19. However, it is uncertain whether these associations are causal. This version ( $v 12$ ) will be the last regular update; however, yearly updates may continue as new evidence becomes available.

v7 of this living review article has been published in $\underline{\text { Addiction }}$

Introduction

COVID-19 is a respiratory disease caused by the SARS-CoV-2 virus. Large age and gender differences in case severity and mortality have been observed in the ongoing COVID-19 pandemic (Guan, $\mathrm{Ni}$, et al., 2020); however, these differences are currently unexplained. SARS-CoV-2 enters epithelial cells through the angiotensin-converting enzyme 2 (ACE-2) receptor (Hoffmann et al., 2020). Some evidence suggests that gene expression and subsequent receptor levels are elevated in the airway and oral epithelium of current smokers (Brake et al., 2020; G. Cai, 2020), which could put smokers at higher risk of contracting SARS-CoV-2. Other studies, however, suggest that nicotine downregulates the ACE-2 receptor (Oakes et al., 2018). These uncertainties notwithstanding, both former and current smoking is known to increase the risk of respiratory viral (Abadom et al., 2016; Denholm et al., 2010) and bacterial (Almirall et al., 1999; Feldman \& Anderson, 2013) infections and is associated with worse outcomes once infected. Cigarette smoke reduces the respiratory immune defence through peri-bronchiolar inflammation and fibrosis, impaired mucociliary clearance and disruption of the respiratory epithelium (Dye \& Adler, 1994). There is also reason to believe that behavioural factors (e.g. regular hand-to-mouth movements) involved in smoking may increase SARS CoV-2 infection and transmission in current smokers. However, early data from the COVID-19 pandemic have not provided clear evidence for a negative impact of current or former smoking on SARS-CoV-2 infection or COVID-19 disease outcomes, such as hospitalisation or mortality (Vardavas \& Nikitara, 2020). It has also been hypothesised that nicotine might protect against a hyperinflammatory response to SARS-CoV-2 infection, which may lead to adverse outcomes in patients with COVID-19 disease (Farsalinos, Niaura, et al., 2020).

There are several reviews that fall within the scope of smoking and COVID-19 (Alqahtani et al., 2020; Berlin et al., 2020; Emami et al., 2020; Farsalinos, Barbouni, et al., 2020; Grundy et al., 2020; Patanavanich \& Glantz, 2020; Vardavas \& Nikitara, 2020). We aimed to produce a rapid synthesis of available evidence pertaining to the rates of infection, hospitalisation, disease severity and mortality from SARS-CoV-2/COVID-19 stratified by smoking status. Given the increasing availability of data on this topic, this was set up in March 2020 as a living review with regular updates.

\section{Methods}

Study design

This is a living evidence review which is updated as new evidence becomes available (Elliott et al., 2014). We adopted recommended best practice for rapid evidence reviews, which involved limiting the search to main databases and having one reviewer extract the data and another verify (Tricco et al., 2015). This study was not pre-registered but evolved from a report written for a UK medical society (Simons, Brown, et al., 2020). The most recent version of this living review is available here. Version 7 of this living review has been published in a peer-reviewed journal (Simons, Shahab, et al., 2020). A completed Preferred Reporting Items for Systematic Reviews and Meta-Analyses (PRISMA) checklist is included in Supplementary file 1. This version (v12) will be the last regular 
update; however, yearly updates may continue as new, high-quality studies (e.g. large, representative/random population studies or experimental studies) become available.

Eligibility criteria

Studies were included if they:

1. Were primary research studies using experimental (e.g. randomised controlled trial), quasi-experimental (e.g. pre- and post-test) or observational (e.g. case-control, retrospective cohort, prospective cohort) study designs;

2. Included adults aged $16+$ years;

3. Recorded as outcome i) results of a SARS-CoV-2 diagnostic test (including antibody assays) in community or hospitalised populations, ii) clinical diagnosis of COVID-19 in community or hospitalised populations, iii) hospitalisation with COVID-19 in community populations testing positive for SARS-CoV-2, iv) severity of COVID-19 disease in hospitalised populations or v) mortality from COVID-19 in community or hospitalised populations; Reported any of the outcomes of interest by self-reported or biochemically verified smoking status (e.g. current smoker, former smoker, never smoker) or current vaping and nicotine replacement therapy (NRT) use;

4. Were available in English;

5. Were published in a peer-reviewed journal, as a pre-print or a public health report by reputable bodies (e.g. governments, scientific societies).

Search strategy

The following terms were searched for in Ovid MEDLINE (2019-search date) as free text or Medical Subject Headings:

1. Tobacco Smoking/ or Smoking Cessation/ or Water Pipe Smoking/ or Smoking/ or Smoking Pipes/ or Cigar Smoking/or Smoking Prevention/ or Cigarette Smoking/ or smoking.mp. or Pipe Smoking/ or Smoking, Non-Tobacco Products/ or Smoking Water Pipes/

2. Nicotine/ or nicotine.mp. or Electronic Nicotine Delivery Systems/ or Nicotine Chewing Gum/

3. vaping.mp. or Vaping/

4. 1 or 2 or 3

5. Coronavirus/ or Severe Acute Respiratory Syndrome/ or Coronavirus Infections/ or covid.mp.

6. 4 and 5

The following terms were searched for in titles, abstracts and full texts in medRxiv (no time limitations):

1. covid (this term captures both covid and SARS-CoV-2) AND smoking

2. covid AND nicotine

3. covid AND vaping

Additional articles/reports of interest were identified through mailing lists, Twitter, the International Severe Acute Respiratory and Emerging Infection Consortium (ISARIC) and the US Centers for Disease Control and Prevention (CDC). Where updated versions of pre-prints or public health reports were available, old versions were superseded.

Selection of studies

One reviewer screened titles, abstracts and full texts against the inclusion criteria.

Data extraction

Data were extracted by one reviewer and verified (i.e. independently checked against pre-prints and published reports) by another on i) author (year); ii) date published; iii) country; iv) study design; v) study setting; vi) sample size; vii) sex; viii) age; ix) smoking status (e.g. current, former, never, not stated, missing) and whether it was biochemically verified; $x$ ) use of alternative nicotine products (e.g. e-cigarettes); xi) SARS-CoV-2 testing; xii) SARS-CoV-2 infection; xiii) diagnosis of COVID-19; xiv) hospitalisation with COVID-19; xv) disease severity in those hospitalised with COVID-19; xvi) mortality; xvii) adjustment of smoking specific risk estimates for relevant covariates (e.g. age, sex); and xviii) whether a representative or random sampling method was used.

Quality appraisal

The quality of included studies was assessed to determine suitability for inclusion in meta-analyses. Studies were judged as 'good' quality if they: i) had <20\% missing data on smoking status and used a reliable self-report measure that distinguished between current, former and never smoking status; AND ii) used biochemical verification of smoking status and reported results from adjusted analyses; OR reported data from a representative/random sample. Studies were rated as 'fair' if they fulfilled only criterion i) and were otherwise rated as 'poor.' The quality appraisal was conducted by one reviewer and verified by a second.

Evidence synthesis

A narrative synthesis was conducted. Data from 'good' and 'fair' quality studies were pooled in R v.3.6.3 (Team, 2013). In a living review where new data are regularly added to the analyses, it may be more appropriate to use a Bayesian (as opposed to frequentist) approach where prior knowledge is used in combination with new data to estimate a posterior risk distribution. A Bayesian approach mitigates the issue of performing multiple statistical tests, which can inflate family-wise error. A series of random-effects hierarchical Bayesian meta-analyses were performed with the brms (Bürkner, 2017) package to estimate the relative risk for each comparison with accompanying $95 \%$ credible intervals (Crls). We first defined prior distributions for the true pooled effect size ( $\mu$ ) and the between-study heterogeneity $(\mathrm{T})$, with $\mu$ specified as a normal distribution with a mean equal to the derived point estimate from each comparison of interest in the immediately preceding version of this living review, and $\mathrm{T}$ specified as a half-Cauchy distribution with a mean of 0 and standard deviation of 1 . The half-Cauchy distribution was selected to reflect prior knowledge that higher levels of between-study heterogeneity are more likely than lower levels. Markov Chain Monte Carlo methods (20,000 burn-ins followed by 80,000 iterations) were then used to generate a risk distribution for each study, in addition to a pooled effect for the posterior risk distribution. We report forest plots with the pooled effect for the posterior risk distribution displayed as the median relative risk with an accompanying $95 \%$ Crls. We used the empirical cumulative distribution function (ECDF) to estimate the probability of there being a $10 \%$ reduction or $10 \%$ increase in relative risk (RR) (i.e. RR $\geq 1.1$ or RR $\leq 0.9$ ). Due to a lack of indication as to what constitutes a clinically or epidemiologically meaningful effect (e.g. with regards to onward disease transmission or requirements for intensive care beds), we deemed a $10 \%$ change in risk as small but important. Where data were inconclusive (as indicated by Crls crossing RR $=1.0$ ), to disambiguate whether data favoured no effect or there 
being a small but important association, we estimated whether there was $\geq 75 \%$ probability of $R R \geq 1.1$ or $R R \leq 0.9$.

Two sensitivity analyses were performed. First, a minimally informative prior for $\mu$ was specified as a normal distribution with a mean of 0 and standard deviation of 1 and t as described above. Second, an informative prior as described above for $\mu$ was used with т specified as a half-Cauchy distribution with a mean of 0.3 and standard deviation of 1 to reflect greater between-study heterogeneity.

To aid in the visualisation of smoking prevalence in the included studies, the weighted mean prevalence of current and former smoking was calculated for countries with $\geq 3$ studies and plotted for comparison with national prevalence estimates. It should be noted that prevalence estimates in the included studies were not adjusted for age, sex, socioeconomic position, or geographic region within countries.

Results

In the current review version (v12) with searches up to 2021-07-18, a total of 1940 records were identified, with 547 studies included in a narrative synthesis and 87 studies included in meta-analyses (see Figure 1).

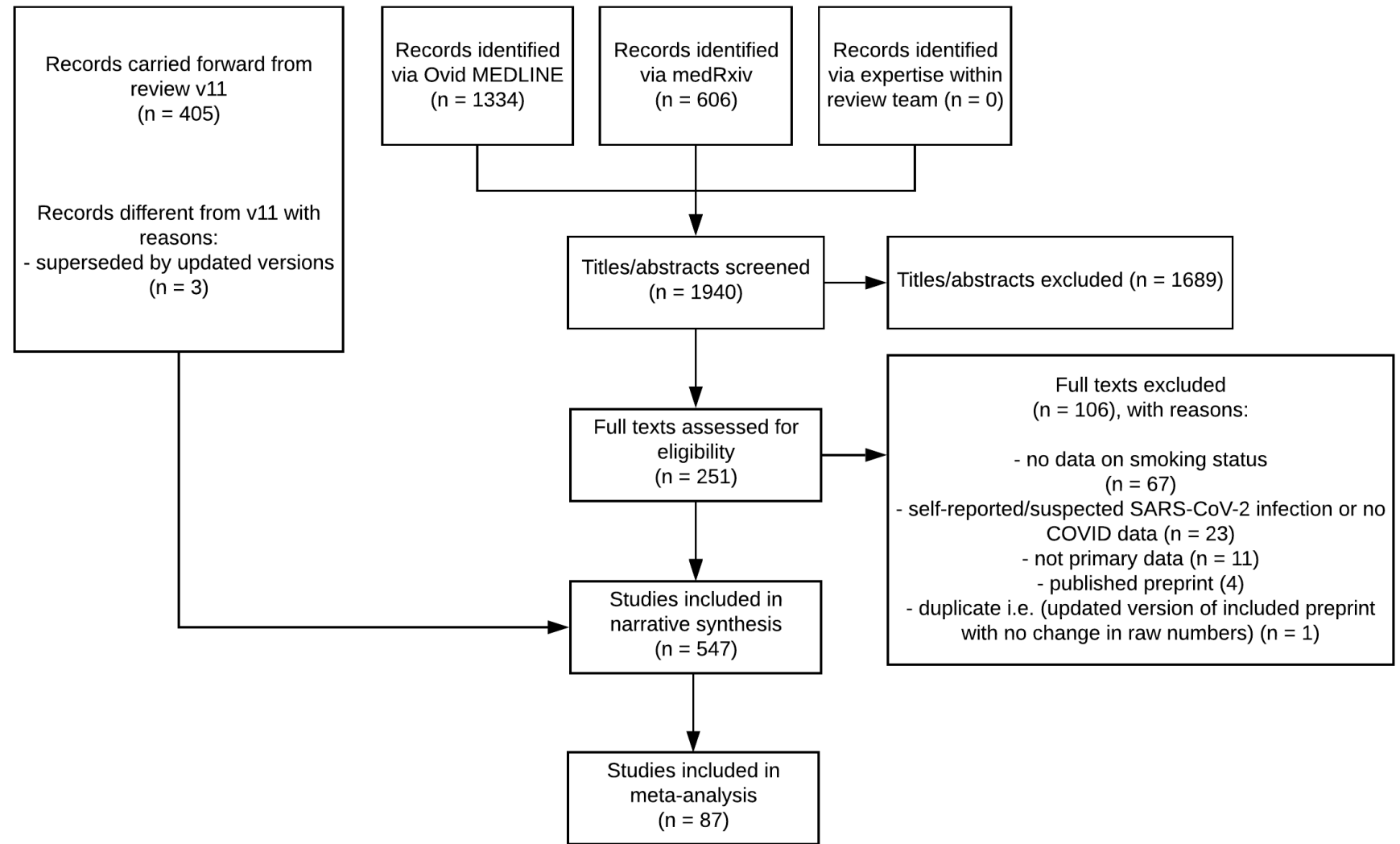

Figure 1. PRISMA flow diagram of included studies.

Characteristics of included studies are presented in Table 1. Studies were conducted across 51 countries. 139 studies were conducted in the USA, 76 in China, 63 in the UK, 32 in Spain, 26 in Italy, 25 in France, 21 in Mexico, 18 in multiple countries, 12 in Switzerland, 11 in Iran, 10 in Turkey, 9 in Brazil, with 8 in India and 97 studies from 39 further countries. The majority of studies used observational designs (see Supplementary table S1). 326 (59.6\%) were conducted in hospital settings, 129 studies (23.6\%) included individuals from community and hospital settings, 88 studies (16.1\%) were conducted exclusively in the community, with one study each conducted in a homeless shelter and a quarantine centre, and one study that did not state the study setting. Studies had a median of 576 (interquartile range $=170-2810$ ) participants. The majority of studies $(58.1 \%)$ used reverse transcriptase polymerase chain reaction (RT-PCR) for confirmation of SARS-CoV-2 infection, $9.7 \%$ used an antibody test to confirm prior infection and $4.4 \%$ of studies relied on a combination of RT-PCR, antigen or antibody assays.

\section{Smoking status}

Categorisation of smoking status was heterogeneous (see Table 1). 296 (54.1\%) studies collected data on smoking status through routine electronic health records (EHRs), 211 (38.6\%) studies used a bespoke case report form for COVID-19 and 40 (7.3\%) studies did not state the source for information on smoking status. None of the studies verified smoking status biochemically. Notably, only $171(31.3 \%)$ studies reported current, former and never smoking status (see Supplementary table S2a), with a further 26 studies reporting only ever and never smoking status (see Supplementary table S2b). The remaining 336 studies reported current, current/former or current and former smoking status but did not explicitly state whether remaining participants were never smokers or if data were missing on smoking status (see Supplementary table S2c). 188 studies explicitly reported the proportion with missing data on smoking status, which ranged from $0 \%$ to $97.6 \%$.

Use of alternative nicotine products 
Fifteen studies recorded the use of alternative nicotine products in current and/or former smokers but did not report COVID-19 outcomes stratified by alternative nicotine use (see Table 1).

Quality appraisal

Nine studies were performed in random or representative population samples and were rated as 'good' quality, and 131 studies were rated as 'fair' quality, of which 87 studies reported results stratified by smoking status for the outcomes of interest and could be included in meta-analyses. The remaining 407 studies were rated as 'poor' quality (see Table 1).

Table 1. All studies included in narrative review and meta-analysis

\begin{tabular}{|c|c|c|c|c|c|c|c|c|c|c|c|c|c|c|c|c|c|c|c|c|}
\hline ref & Lead author & $\begin{array}{l}\text { Date } \\
\text { published }\end{array}$ & Country & $\begin{array}{l}\text { Sample } \\
\text { size }\end{array}$ & $\begin{array}{l}\text { Study } \\
\text { setting }\end{array}$ & $\begin{array}{l}\text { Median } \\
\text { (IQR) }\end{array}$ & $\begin{array}{l}\text { Female } \\
\%\end{array}$ & $\begin{array}{l}\text { Current } \\
\text { smoker } \\
\%\end{array}$ & $\begin{array}{l}\text { Current } \\
\text { vaper } \\
\%\end{array}$ & $\begin{array}{l}\text { Current } \\
\text { smoker, } \\
\text { current } \\
\text { vaper \% }\end{array}$ & $\begin{array}{l}\text { Current } \\
\text { smoker, } \\
\text { former } \\
\text { vaper \% }\end{array}$ & $\begin{array}{l}\text { Former } \\
\text { smoker } \\
\%\end{array}$ & $\begin{array}{l}\text { Former } \\
\text { smoker, } \\
\text { current } \\
\text { vaper \% }\end{array}$ & $\begin{array}{l}\text { Former } \\
\text { smoker, } \\
\text { former } \\
\text { vaper \% }\end{array}$ & $\begin{array}{l}\text { Current/former } \\
\text { smokers \% }\end{array}$ & $\begin{array}{l}\begin{array}{l}\text { Never } \\
\text { smokers } \\
\%\end{array} \\
\text { \% }\end{array}$ & $\begin{array}{l}\text { Never } \\
\text { smoker, } \\
\text { current } \\
\text { vaper } \%\end{array}$ & $\begin{array}{l}\text { Never } \\
\text { smoker, } \\
\text { former } \\
\text { vaper } \%\end{array}$ & $\begin{array}{l}\text { Never/unknown } \\
\text { smokers \% }\end{array}$ & $\begin{array}{l}\text { Missin } \\
\%\end{array}$ \\
\hline $\begin{array}{l}\text { C. Huang et al. } \\
\text { (2020) }\end{array}$ & Huang, Wang & $\begin{array}{l}2020-01- \\
24\end{array}$ & China & 41 & Hospital & $\begin{array}{l}49(41- \\
58)\end{array}$ & 27.0 & 7.3 & & & - & - & & - & & & & - & - & 92.7 \\
\hline Jie Li et al. (2020) & $\mathrm{Li}$ & $\begin{array}{l}2020-02- \\
12\end{array}$ & China & 17 & Hospital & $\begin{array}{l}45(33- \\
57)\end{array}$ & 47.1 & 17.6 & - & - & - & - & - & - & - & & & - & - & 82.3 \\
\hline $\begin{array}{l}\text { Jin-jin Zhang et al. } \\
\text { (2020) }\end{array}$ & Zhang, Dong & $\begin{array}{l}2020-02- \\
19\end{array}$ & China & 140 & Hospital & $\begin{array}{l}57^{\wedge} \\
(25-87)\end{array}$ & 46.3 & 1.4 & - & & - & 5.0 & - & - & - & & & - & - & 93.6 \\
\hline Yang et al. (2020) & Yang, Yu & $\begin{array}{l}2020-02- \\
24\end{array}$ & China & 52 & Hospital & $\begin{array}{l}60(47- \\
73)\end{array}$ & 37.0 & 3.8 & & & & & & & & & & & & 96.2 \\
\hline $\begin{array}{l}\text { Guan, Ni, et al. } \\
(2020)\end{array}$ & Guan, $\mathrm{Ni}$ & $\begin{array}{l}2020-02- \\
28\end{array}$ & China & 1,099 & Hospital & $\begin{array}{l}47(35- \\
58)\end{array}$ & 41.9 & 12.5 & - & & - & 1.9 & & - & & 84.3 & - & - & - & 1.3 \\
\hline W. Liu et al. (2020) & Liu, Tao & $\begin{array}{l}2020-02- \\
28\end{array}$ & China & 78 & Hospital & $\begin{array}{l}38(33- \\
57)\end{array}$ & 50.0 & - & - & - & - & - & - & - & 6.4 & & & - & - & 93.6 \\
\hline Qi et al. (2020) & Qi & $\begin{array}{l}2020-03- \\
03\end{array}$ & China & 267 & Hospital & $\begin{array}{l}48(35- \\
65)\end{array}$ & 45.2 & 19.9 & - & - & - & - & - & - & - & - & - & - & 80.1 & 0.0 \\
\hline $\begin{array}{l}\text { Y. Huang et al. } \\
(2020)\end{array}$ & Huang, Yang & $\begin{array}{l}2020-03- \\
05\end{array}$ & China & 36 & Hospital & $\begin{array}{l}69(60- \\
78)\end{array}$ & 30.6 & & & & & & & & 11.1 & & & & & 88.9 \\
\hline Xu et al. (2020) & $\mathrm{Xu}$ & $\begin{array}{l}2020-03- \\
08\end{array}$ & China & 53 & Hospital & NA & 47.2 & 11.3 & & & - & & & & & & & & - & 88.7 \\
\hline $\begin{array}{l}\text { F. Zhou et al. } \\
(2020)\end{array}$ & Zhou, Yu & $\begin{array}{l}2020-03- \\
11\end{array}$ & China & 191 & Hospital & $\begin{array}{l}56(46- \\
67)\end{array}$ & 38.0 & 5.8 & - & - & - & - & - & - & - & & & - & - & 94.2 \\
\hline R. Liu et al. (2020) & Liu, Ming & $\begin{array}{l}2020-03- \\
12\end{array}$ & China & 41 & Hospital & $\begin{array}{l}39(30- \\
48)\end{array}$ & 58.5 & 9.8 & - & - & - & - & - & - & - & - & - & - & - & 90.2 \\
\hline Mo et al. (n.d.) & Mo & $\begin{array}{l}2020-03- \\
16\end{array}$ & China & 155 & Hospital & $\begin{array}{l}54(53- \\
66)\end{array}$ & 44.5 & 3.9 & & & & & & & & & & & & 96.1 \\
\hline Y. Shi et al. (2020) & Shi, Yu & $\begin{array}{l}2020-03- \\
18\end{array}$ & China & 487 & Hospital & $\begin{array}{l}46(27- \\
65)\end{array}$ & 46.8 & - & - & - & - & - & - & - & 8.2 & & & - & - & 91.8 \\
\hline $\begin{array}{l}\text { Xiaoli Zhang et al. } \\
\text { (2020) }\end{array}$ & Zhang, Cai & $\begin{array}{l}2020-03- \\
20\end{array}$ & China & 645 & Hospital & NA & 49.1 & 6.4 & - & - & - & - & - & - & - & & & - & - & 93.6 \\
\hline Dong et al. (2020) & Dong, Cao & $\begin{array}{l}2020-03- \\
20\end{array}$ & China & 9 & Hospital & $\begin{array}{l}44(30- \\
46)\end{array}$ & 66.7 & 11.1 & - & - & - & - & - & - & $\cdot$ & - & - & - & - & 88.9 \\
\hline $\begin{array}{l}\text { S. Wan et al. } \\
(2020)\end{array}$ & Wan & $\begin{array}{l}2020-03- \\
21\end{array}$ & China & 135 & Hospital & $\begin{array}{l}47(36- \\
55)\end{array}$ & 46.7 & 6.7 & & & & & & & & & & & & 93.3 \\
\hline X. Jin et al. (2020) & Jin & $\begin{array}{l}2020-03- \\
24\end{array}$ & China & 651 & Hospital & $\begin{array}{l}46(32- \\
60)\end{array}$ & 49.2 & 6.3 & - & - & - & - & - & - & - & & & - & - & 93.7 \\
\hline $\begin{array}{l}\text { R. Wang et al. } \\
(2020)\end{array}$ & Wang, Pan & $\begin{array}{l}2020-03- \\
24\end{array}$ & China & 125 & Hospital & $\begin{array}{l}41(26- \\
66)\end{array}$ & 43.2 & - & - & - & - & - & - & - & 12.8 & - & - & - & & 87.2 \\
\hline Lian et al. (n.d.) & Lian & $\begin{array}{l}2020-03- \\
25\end{array}$ & China & 788 & Hospital & NA & 38.5 & 6.9 & - & - & - & - & - & - & - & - & - & - & - & 93.2 \\
\hline Hu et al. (2020) & $\mathrm{Hu}$ & $\begin{array}{l}2020-03- \\
25\end{array}$ & China & 323 & Hospital & $\begin{array}{l}61^{\wedge} \\
(23-91)\end{array}$ & 48.6 & & & & & & & & 11.8 & & & & & 88.2 \\
\hline $\begin{array}{l}\text { Guan, Liang, et al. } \\
\text { (2020) }\end{array}$ & Guan, Liang & $\begin{array}{l}2020-03- \\
26\end{array}$ & China & 1,590 & Hospital & $\begin{array}{l}49(33- \\
64)\end{array}$ & 42.7 & - & - & - & - & - & - & - & 7.0 & 93.0 & & - & - & 0.0 \\
\hline $\begin{array}{l}\text { T. Chen et al. } \\
\text { (2020) }\end{array}$ & Chen & $\begin{array}{l}2020-03- \\
26\end{array}$ & China & 548 & Hospital & $\begin{array}{l}62(44- \\
70)\end{array}$ & 37.6 & 4.4 & - & - & - & 2.6 & - & - & - & - & - & - & & 93.1 \\
\hline Guo et al. (2020) & Guo & $\begin{array}{l}2020-03- \\
27\end{array}$ & China & 187 & Hospital & $\begin{array}{l}59(45- \\
73)\end{array}$ & 51.3 & 9.6 & & & & & & & & & & & - & 90.4 \\
\hline $\begin{array}{l}\text { CDCMMWR } \\
\text { (2020) }\end{array}$ & Chow (US CDC) & $\begin{array}{l}2020-03- \\
31\end{array}$ & USA & 7,162 & $\begin{array}{l}\text { Community } \\
\text { and } \\
\text { Hospital }\end{array}$ & NA & - & 1.3 & - & - & - & 2.3 & - & - & $\cdot$ & & & - & - & 96.4 \\
\hline $\begin{array}{l}\text { E. S. Kim et al. } \\
\text { (2020) }\end{array}$ & Kim & $\begin{array}{l}2020-04- \\
01\end{array}$ & $\begin{array}{l}\text { South } \\
\text { Korea }\end{array}$ & 28 & Hospital & $\begin{array}{l}43(30- \\
56)\end{array}$ & 46.4 & 17.9 & - & - & - & - & - & - & - & - & - & - & & 82.1 \\
\hline Feng et al. (2020) & Feng & $\begin{array}{l}2020-04- \\
10\end{array}$ & China & 476 & Hospital & $\begin{array}{l}53(40- \\
64)\end{array}$ & 43.1 & 9.2 & - & & & - & - & - & & - & - & - & - & 90.8 \\
\hline $\begin{array}{l}\text { Christopher T. } \\
\text { Rentsch et al. } \\
\text { (2020b) }\end{array}$ & Rentsch & $\begin{array}{l}2020-04- \\
14\end{array}$ & USA & 3,528 & $\begin{array}{l}\text { Community } \\
\text { and } \\
\text { Hospital }\end{array}$ & $\begin{array}{l}66(60- \\
70)\end{array}$ & 4.6 & 27.2 & - & - & - & 30.6 & - & - & - & 36.9 & & - & - & 5.3 \\
\hline Goyal et al. (2020) & Goyal & $\begin{array}{l}2020-04- \\
17\end{array}$ & USA & 393 & Hospital & $\begin{array}{l}62.2 \\
(49-74)\end{array}$ & 39.3 & 5.1 & - & - & - & - & - & - & - & - & - & - & - & 94.9 \\
\hline $\begin{array}{l}\text { K. I. Zheng et al. } \\
\text { (2020) }\end{array}$ & Zheng, Gao & $\begin{array}{l}2020-04- \\
19\end{array}$ & China & 66 & Hospital & $\begin{array}{l}47^{n} \\
(\mathrm{NA})\end{array}$ & 25.8 & 12.1 & & & & & & & & & & & & 87.9 \\
\hline Gold et al. (2020) & Gold (US CDC) & $\begin{array}{l}2020-04- \\
20\end{array}$ & USA & 305 & Hospital & NA & 50.5 & 5.2 & & - & & & & - & - & & & & & 94.8 \\
\hline $\begin{array}{l}\text { Argenziano et al. } \\
\text { (2020) }\end{array}$ & Argenziano & $\begin{array}{l}2020-04- \\
22\end{array}$ & USA & 1,000 & Hospital & $\begin{array}{l}63(50- \\
75)\end{array}$ & 40.4 & 4.9 & - & - & - & 17.9 & - & - & - & 77.2 & & - & - & 0.0 \\
\hline $\begin{array}{l}\text { Richardson et al. } \\
\text { (2020) }\end{array}$ & Richardson & $\begin{array}{l}2020-04- \\
22\end{array}$ & USA & 5,700 & Hospital & $\begin{array}{l}63(52- \\
75)\end{array}$ & 39.7 & - & - & - & - & - & - & - & 9.8 & 52.8 & - & - & - & 37.4 \\
\hline Fontanet et al. & $\ldots$ & 2020-04- & r..... & $m+$ & Community & $37(16-$ & - & th & & & & & & & & & & & $n n$ & $n n$ \\
\hline
\end{tabular}




\begin{tabular}{|c|c|c|c|c|c|c|c|c|c|c|c|c|c|c|c|c|c|c|c|c|}
\hline (2020) & runlianter & 23 & rranlice & 001 & $\begin{array}{l}\text { anu } \\
\text { Hospital }\end{array}$ & 47) & $0<. \cup$ & 10.4 & 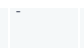 & & & & $\cdot$ & & & & $\cdot$ & & 09.0 & u.u \\
\hline P. Shi et al. (2020) & Shi, Ren & $\begin{array}{l}2020-04- \\
23\end{array}$ & China & 134 & Hospital & $\begin{array}{l}46(34- \\
58)\end{array}$ & 51.5 & & & & & & & & 10.4 & & & & & 89.5 \\
\hline $\begin{array}{l}\text { Hadjadj et al. } \\
\text { (2020) }\end{array}$ & Hadjadj & $\begin{array}{l}2020-04- \\
23\end{array}$ & France & 50 & Hospital & $\begin{array}{l}55(50- \\
63)\end{array}$ & 22.0 & 2.0 & & & & 18.0 & & & & 80.0 & & & & 0.0 \\
\hline Liao et al. (2020) & Liao, Feng & $\begin{array}{l}2020-04- \\
24\end{array}$ & China & 1,848 & Hospital & $\begin{array}{l}55(48- \\
61)\end{array}$ & 54.7 & & & & & & & & 0.4 & & & & & 99.6 \\
\hline $\begin{array}{l}\text { Gil-Agudo et al. } \\
\text { (2020) }\end{array}$ & Gil-Agudo & $\begin{array}{l}2020-04- \\
24\end{array}$ & Spain & 7 & Hospital & $\begin{array}{l}68(34- \\
75)\end{array}$ & 28.6 & & - & & - & & $\cdot$ & & 42.9 & 57.1 & & & & 0.0 \\
\hline Q. Yao et al. (n.d.) & Yao & $\begin{array}{l}2020-04- \\
24\end{array}$ & China & 108 & Hospital & $\begin{array}{l}52(37- \\
58)\end{array}$ & 60.2 & 3.7 & & & & & & & & & & & & 96.3 \\
\hline $\begin{array}{l}\text { Zuo, Yalavarthi, et } \\
\text { al. (2020) }\end{array}$ & Zuo, Yalavarthi & $\begin{array}{l}2020-04- \\
24\end{array}$ & USA & 50 & Hospital & $\begin{array}{l}61(46- \\
76)\end{array}$ & 34.0 & & & & & & & & 36.0 & & & & & 64.0 \\
\hline $\begin{array}{l}\text { Solis \& Carreño } \\
\text { (2020) }\end{array}$ & Solis & $\begin{array}{l}2020-04- \\
25\end{array}$ & Mexico & 650 & Hospital & $46(\mathrm{NA})$ & 42.1 & 9.4 & & & & & & & & & & & & 90.6 \\
\hline Yu et al. (2020) & Yu, Cai & $\begin{array}{l}2020-04- \\
27\end{array}$ & China & 95 & Hospital & NA & 44.2 & 8.4 & & & & & & & & & & & & 91.6 \\
\hline Ziehr et al. (2020) & Ziehr & $\begin{array}{l}2020-04- \\
29\end{array}$ & USA & 66 & Hospital & $\begin{array}{l}58(23- \\
87)\end{array}$ & 35.0 & & & & & & & & 33.3 & 63.6 & & & & 3.0 \\
\hline $\begin{array}{l}\text { Yi Zheng et al. } \\
\text { (2020) }\end{array}$ & Zheng, Xiong & $\begin{array}{l}2020-04- \\
30\end{array}$ & China & 73 & Hospital & $\begin{array}{l}43^{n} \\
(\mathrm{NA})\end{array}$ & 45.2 & & & & & & & & 11.0 & 89.0 & & & & 0.0 \\
\hline Kalan et al. (2020) & Kalan & $\begin{array}{l}2020-05- \\
01\end{array}$ & Iran & 193 & Hospital & $\begin{array}{l}52.6^{\wedge} \\
(37-67)\end{array}$ & 36.3 & 7.3 & & & & & & & & 85.0 & & & & 7.8 \\
\hline Kolin et al. (2020) & Kolin & $\begin{array}{l}2020-05- \\
05\end{array}$ & UK & 1,474 & $\begin{array}{l}\text { Community } \\
\text { and } \\
\text { Hospital }\end{array}$ & $\begin{array}{l}58(49- \\
67)\end{array}$ & 46.6 & 14.5 & & & & 40.2 & & & & 44.6 & & & & 0.8 \\
\hline $\begin{array}{l}\text { Borobia et al. } \\
\text { (2020) }\end{array}$ & Borobia & $\begin{array}{l}2020-05- \\
06\end{array}$ & Spain & 2,226 & Hospital & $\begin{array}{l}61(46- \\
78)\end{array}$ & 52.0 & 7.1 & & & & & & & & & & & & 93.0 \\
\hline $\begin{array}{l}\text { Giacomelli et al. } \\
\text { (2020) }\end{array}$ & Giacomelli & $\begin{array}{l}2020-05- \\
06\end{array}$ & Italy & 233 & Hospital & $\begin{array}{l}61(50- \\
72)\end{array}$ & 31.9 & & & & & & & & 30.0 & 70.0 & & & & 0.0 \\
\hline Shah et al. (2020) & Shah & $\begin{array}{l}2020-05- \\
06\end{array}$ & USA & 316 & Hospital & $\begin{array}{l}63(43- \\
72)\end{array}$ & 48.1 & 16.5 & & & & 17.7 & & & & 42.1 & & & & 23.7 \\
\hline $\begin{array}{l}\text { Williamson et al. } \\
\text { (2020) }\end{array}$ & $\begin{array}{l}\text { The OpenSAFELY } \\
\text { Collaborative }\end{array}$ & $\begin{array}{l}2020-05- \\
07\end{array}$ & UK & $17,425,445$ & $\begin{array}{l}\text { Community } \\
\text { and } \\
\text { Hospital }\end{array}$ & NA & 50.1 & 17.0 & & & & 32.9 & & & & 45.9 & & & & 4.2 \\
\hline $\begin{array}{l}\text { Allenbach et al. } \\
\text { (2020) }\end{array}$ & Allenbach & $\begin{array}{l}2020-05- \\
08\end{array}$ & France & 152 & Hospital & $\begin{array}{l}77(60- \\
83)\end{array}$ & 31.1 & & & - & - & & & & 6.6 & & & & & 93.4 \\
\hline $\begin{array}{l}\text { Robilotti et al. } \\
\text { (2020) }\end{array}$ & Robilotti & $\begin{array}{l}2020-05- \\
08\end{array}$ & USA & 423 & Hospital & NA & 50.0 & 2.1 & & - & - & 37.6 & & & & 58.6 & & & & 1.6 \\
\hline $\begin{array}{l}\text { Lubetzky et al. } \\
\text { (2020) }\end{array}$ & Lubetzky & $\begin{array}{l}2020-05- \\
08\end{array}$ & USA & 54 & Hospital & $\begin{array}{l}57(29- \\
83)\end{array}$ & 62.0 & & & & & & & & 22.2 & & & & & 77.8 \\
\hline Yin et al. (2020) & Yin, Yang & $\begin{array}{l}2020-05- \\
10\end{array}$ & China & 106 & Hospital & $\begin{array}{l}73 \text { (61- } \\
85)\end{array}$ & 39.6 & & & & & & & & 17.0 & & & & & 83.0 \\
\hline Rica et al. (2020) & de la Rica & $\begin{array}{l}2020-05- \\
11\end{array}$ & Spain & 48 & Hospital & $\begin{array}{l}66^{\wedge} \\
(33-88)\end{array}$ & 33.0 & & & & & & & & 20.8 & & & & & 79.2 \\
\hline Cho et al. (2020) & Cho & $\begin{array}{l}2020-05- \\
11\end{array}$ & UK & 1,331 & $\begin{array}{l}\text { Community } \\
\text { and } \\
\text { Hospital }\end{array}$ & NA & 49.2 & 19.0 & & & & 27.0 & & & & 54.0 & & & & 0.0 \\
\hline $\begin{array}{l}\text { Yanover et al. } \\
(2020)\end{array}$ & Yanover & $\begin{array}{l}2020-05- \\
13\end{array}$ & Israel & 4,353 & $\begin{array}{l}\text { Community } \\
\text { and } \\
\text { Hospital }\end{array}$ & $\begin{array}{l}35(22- \\
54)\end{array}$ & 44.5 & 11.8 & & & & 3.0 & & & & 85.2 & & & & 0.0 \\
\hline $\begin{array}{l}\text { Hamer et al. } \\
\text { (2020) }\end{array}$ & Hamer & $\begin{array}{l}2020-05- \\
13\end{array}$ & UK & 387,109 & Hospital & $\begin{array}{l}56.2 \\
(48-64)\end{array}$ & 55.1 & 9.7 & & & & 34.8 & & & & 55.5 & & & & 0.0 \\
\hline $\begin{array}{l}\text { Targher et al. } \\
(2020)\end{array}$ & Targher & $\begin{array}{l}2020-05- \\
13\end{array}$ & China & 339 & Hospital & $\begin{array}{l}48.4^{4} \\
\text { (NA) }\end{array}$ & 52.8 & 8.3 & & & & & & & & & & & & 91.7 \\
\hline $\begin{array}{l}\text { Carrillo-Vega et al. } \\
\text { (2020) }\end{array}$ & Carillo-Vega & $\begin{array}{l}2020-05- \\
14\end{array}$ & Mexico & 10,544 & $\begin{array}{l}\text { Community } \\
\text { and } \\
\text { Hospital }\end{array}$ & $\begin{array}{l}46.5^{\wedge} \\
(30-62)\end{array}$ & 42.3 & 8.9 & & & & & & & & & & & & 91.1 \\
\hline $\begin{array}{l}\text { Regina et al. } \\
(2020)\end{array}$ & Regina & $\begin{array}{l}2020-05- \\
14\end{array}$ & Switzerland & 200 & Hospital & $\begin{array}{l}70(55- \\
81)\end{array}$ & 40.0 & 4.5 & & & & & & & & & & & & 95.5 \\
\hline $\begin{array}{l}\text { Almazeedi et al. } \\
(2020)\end{array}$ & Almazeedi & $\begin{array}{l}2020-05- \\
15\end{array}$ & Kuwait & 1,096 & Hospital & $\begin{array}{l}41(25- \\
57)\end{array}$ & 19.0 & 4.0 & & & & & & & & & & & 96.0 & 0.0 \\
\hline $\begin{array}{l}\text { Lusignan et al. } \\
\text { (2020) }\end{array}$ & de Lusignan & $\begin{array}{l}2020-05- \\
15\end{array}$ & UK & 3,802 & Community & $\begin{array}{l}58(34- \\
73)\end{array}$ & 57.6 & 10.9 & & & & 46.1 & & & & 29.6 & & & & 13.4 \\
\hline $\begin{array}{l}\text { Palaiodimos et al. } \\
\text { (2020) }\end{array}$ & Palaiodimos & $\begin{array}{l}2020-05- \\
15\end{array}$ & USA & 200 & Hospital & $\begin{array}{l}64(50- \\
73.5)\end{array}$ & 51.0 & & & & & & & & 32.5 & 67.5 & & & & 0.0 \\
\hline $\begin{array}{l}\text { Meija-Vilet et al. } \\
\text { (2020) }\end{array}$ & Mejia-Vilet & $\begin{array}{l}2020-05- \\
16\end{array}$ & Mexico & 329 & Hospital & $\begin{array}{l}49(41- \\
60)\end{array}$ & 36.0 & & & & & & & & 7.0 & & & & & 93.0 \\
\hline $\begin{array}{l}\text { C. Chen et al. } \\
(2020)\end{array}$ & Chen, Jiang & $\begin{array}{l}2020-05- \\
16\end{array}$ & China & 135 & Hospital & NA & 42.2 & & & & & & & & 9.6 & & & & & 90.4 \\
\hline $\begin{array}{l}\text { Jiong Liet al. } \\
(2020)\end{array}$ & Li, Chen & $\begin{array}{l}2020-05- \\
16\end{array}$ & China & 1,008 & Hospital & $\begin{array}{l}55(44- \\
65)\end{array}$ & 43.6 & 5.7 & & & & & & & & & & & & 94.3 \\
\hline $\begin{array}{l}\text { Valenti et al. } \\
\text { (2020) }\end{array}$ & Valenti & $\begin{array}{l}2020-05- \\
18\end{array}$ & Italy & 789 & Community & $\begin{array}{l}40.7 n \\
(\mathrm{NA})\end{array}$ & 35.0 & 25.9 & & & & & & & & & & & & 74.1 \\
\hline Feuth et al. (2020) & Feuth & $\begin{array}{l}2020-05- \\
18\end{array}$ & Finland & 28 & Hospital & $\begin{array}{l}56(47- \\
72)\end{array}$ & 46.0 & 10.7 & & & & 28.6 & & & & 60.7 & & & & 0.0 \\
\hline Ge et al. (2020) & $\mathrm{Ge}$ & $\begin{array}{l}2020-05- \\
18\end{array}$ & China & 51 & Hospital & $\begin{array}{l}70(58- \\
79)\end{array}$ & 27.5 & 13.7 & & & & & & & & & & & & 86.3 \\
\hline $\begin{array}{l}\text { Parrotta et al. } \\
(2020)\end{array}$ & Parrotta & $\begin{array}{l}2020-05- \\
18\end{array}$ & USA & 76 & $\begin{array}{l}\text { Community } \\
\text { and } \\
\text { Hospital }\end{array}$ & $\begin{array}{l}44.9 \\
(13-71)\end{array}$ & 61.8 & 2.6 & & & & 26.3 & & & & 68.4 & & & & 2.6 \\
\hline Shekhar et a & & 2020-05- & $\because n$ & $m$ & . & 55.5 & & $\ldots$ & & & & & & & & & & & & m \\
\hline
\end{tabular}




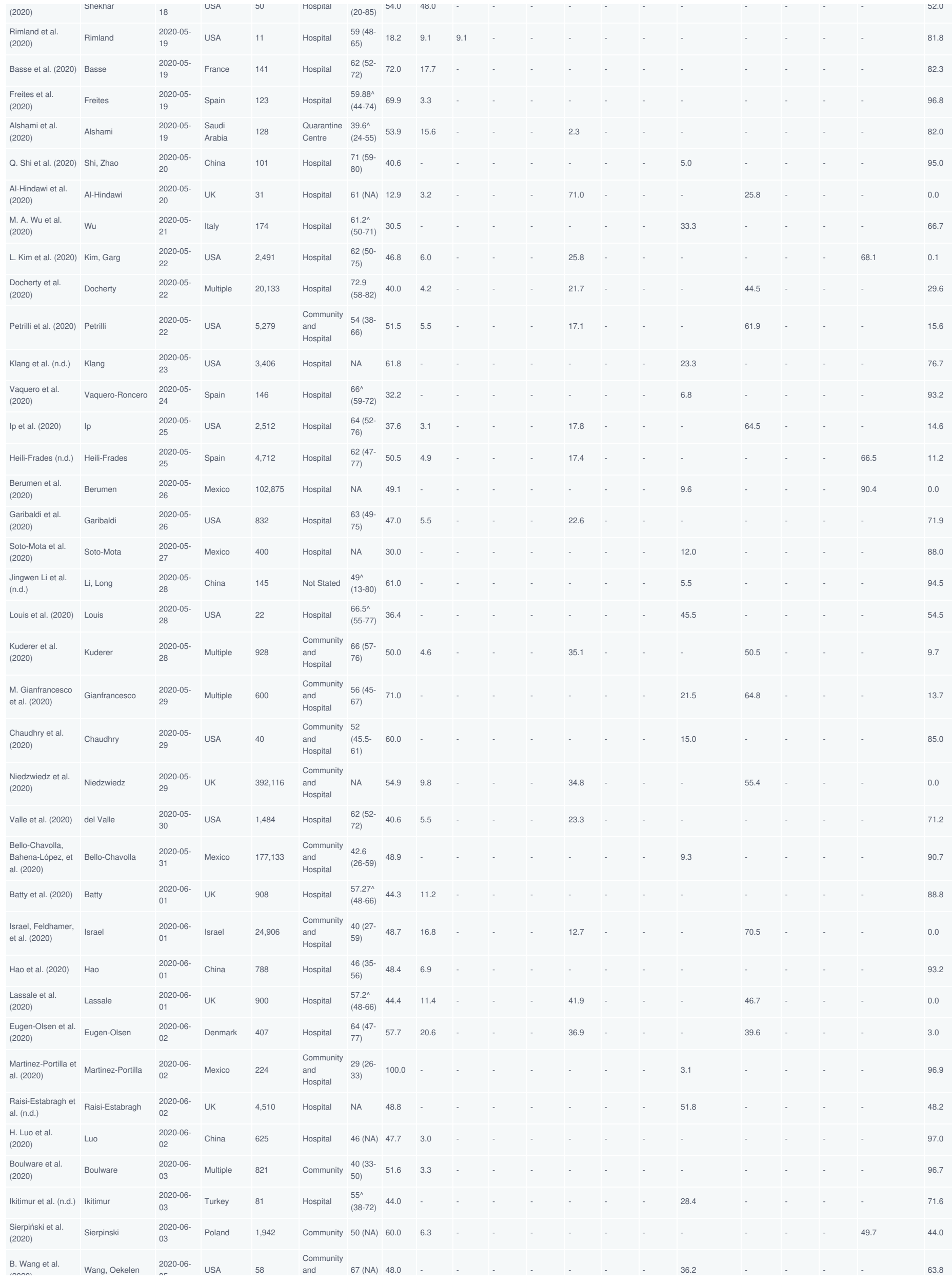




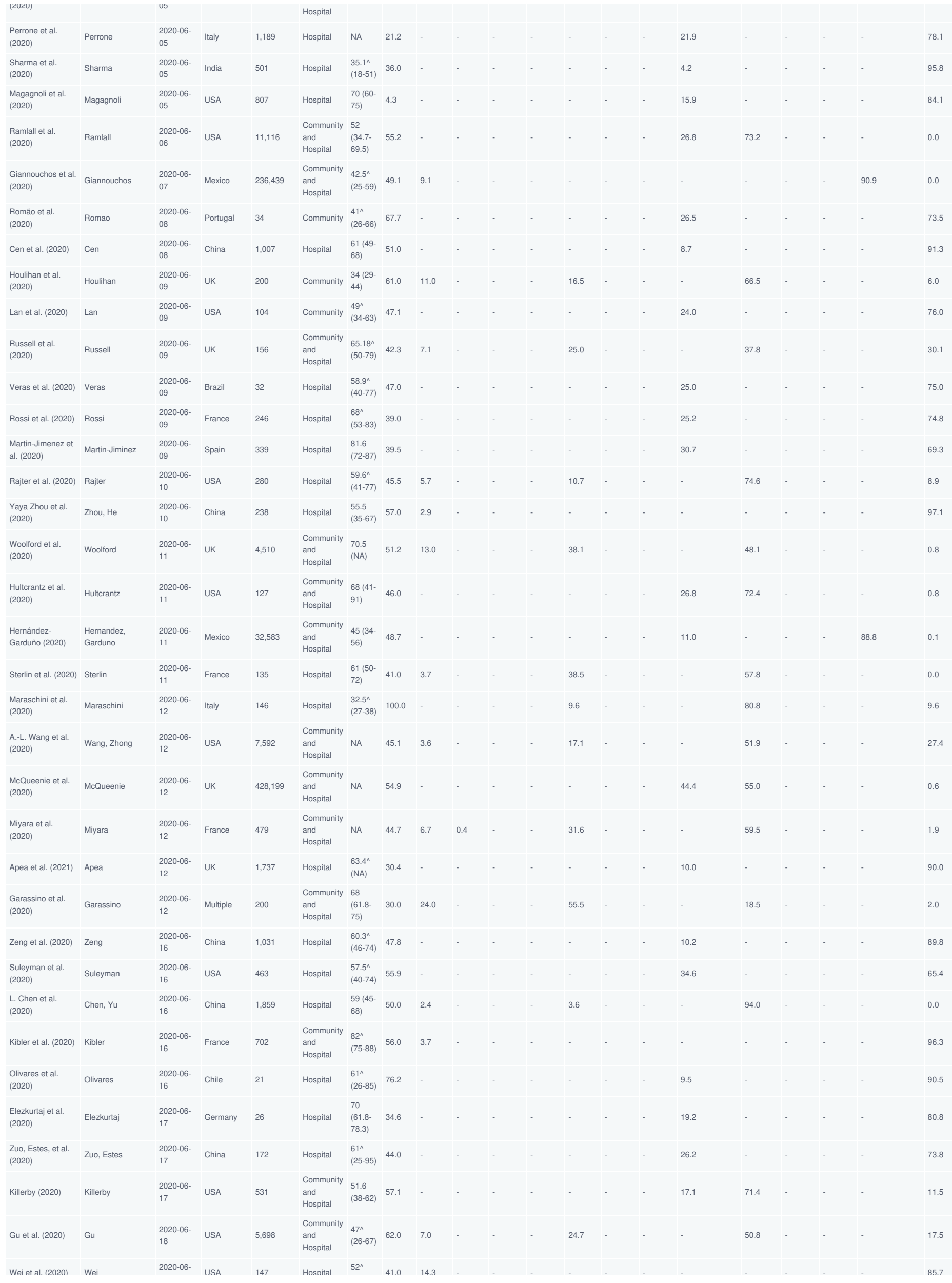




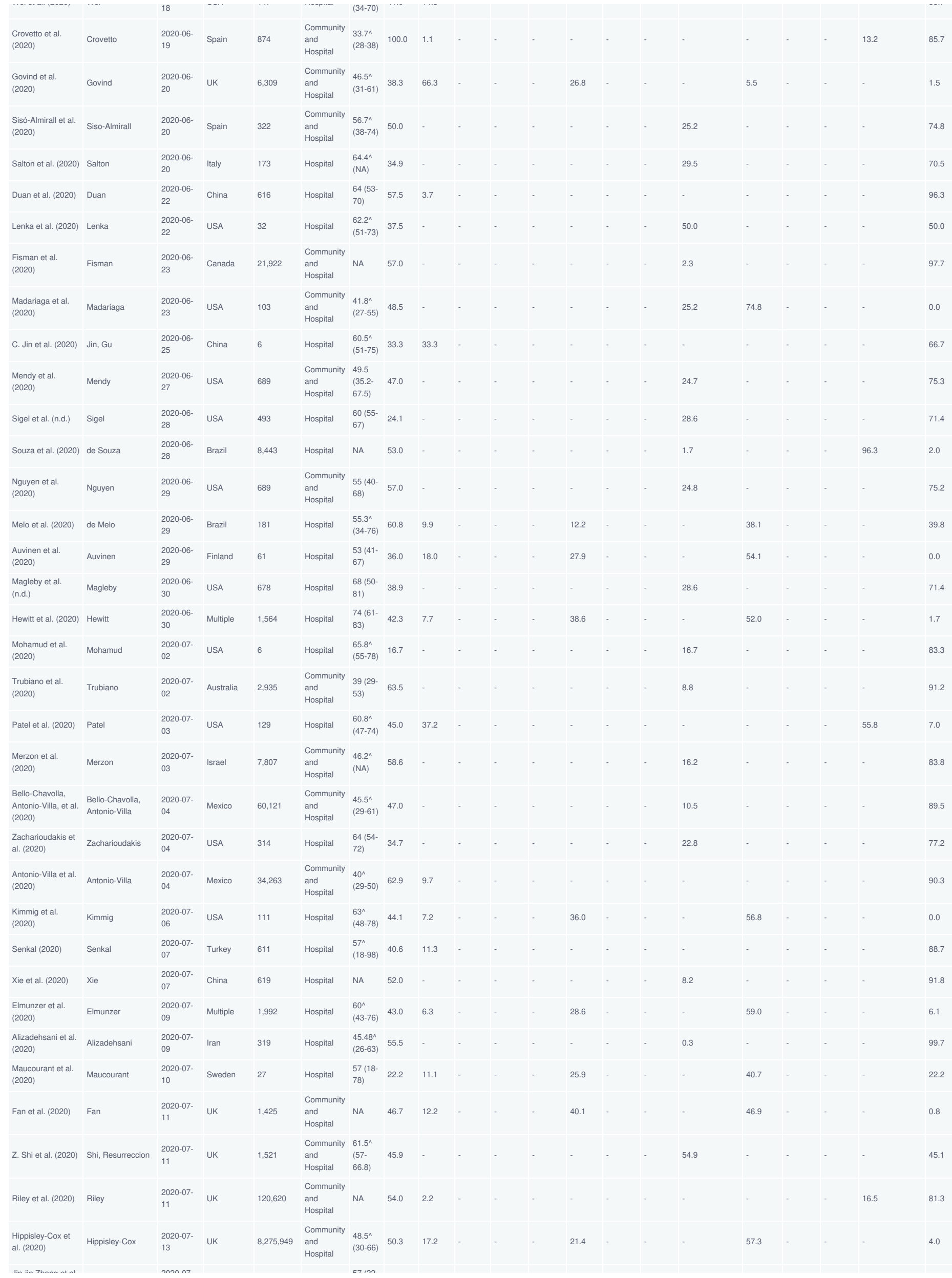




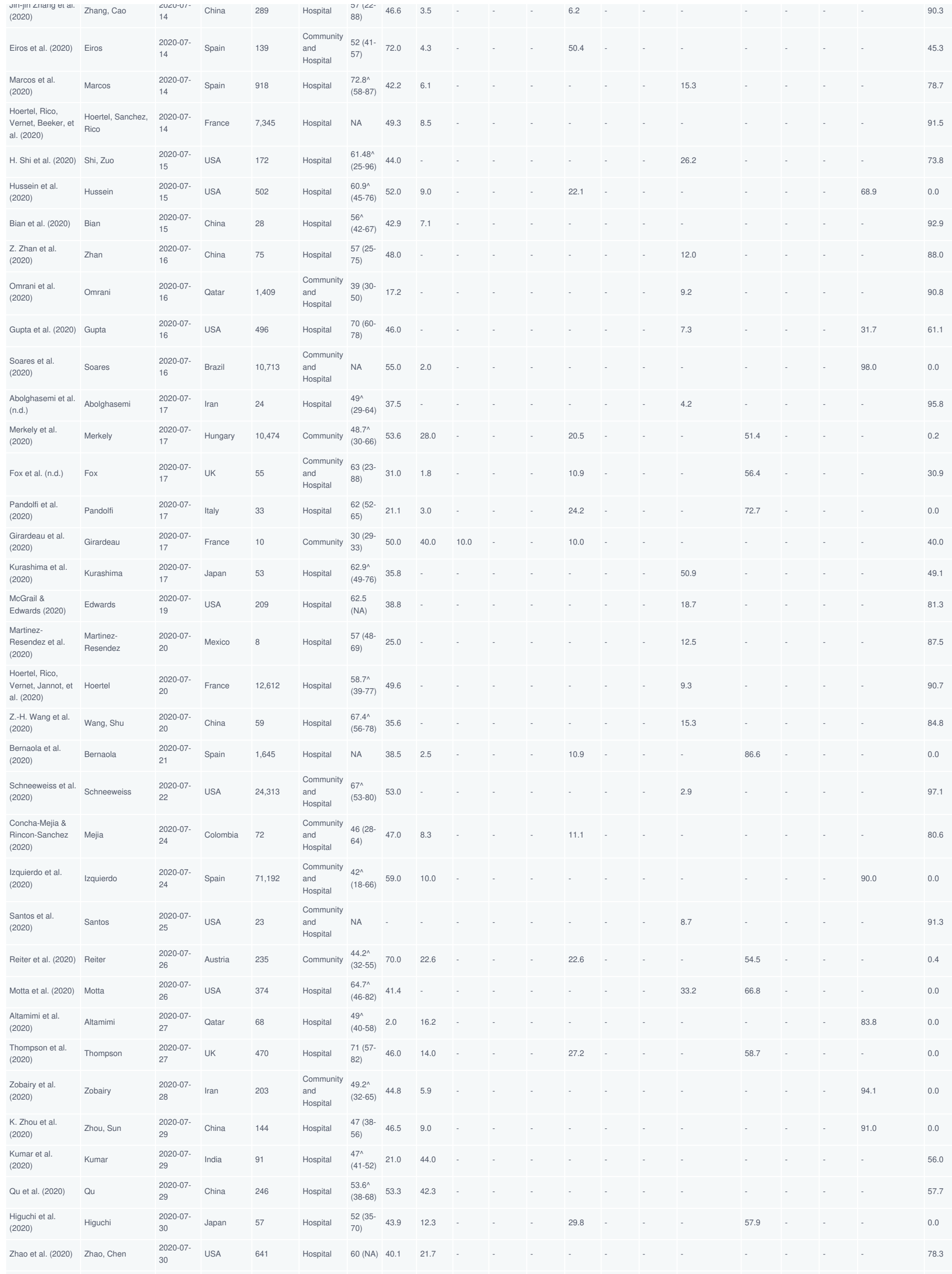




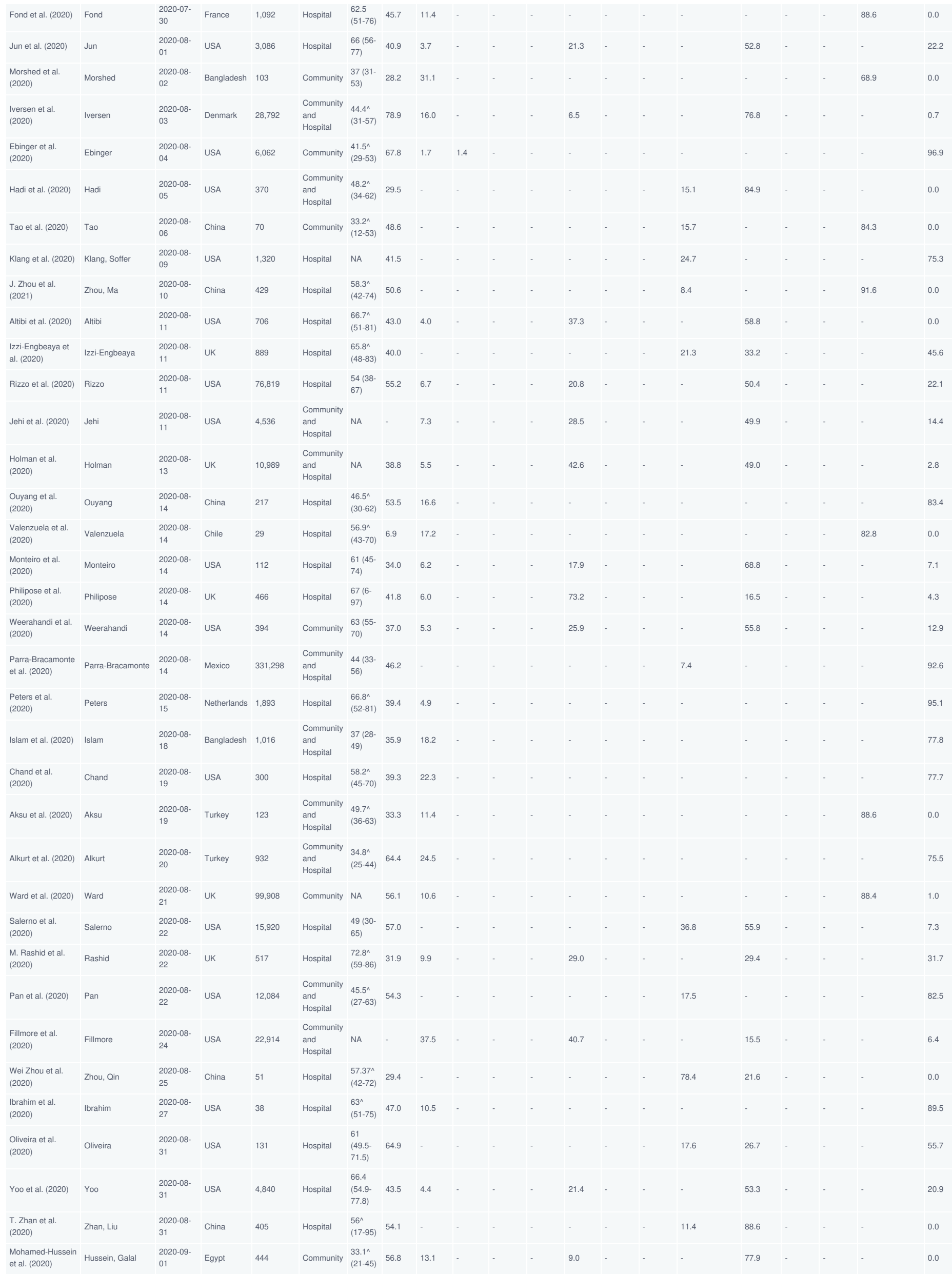




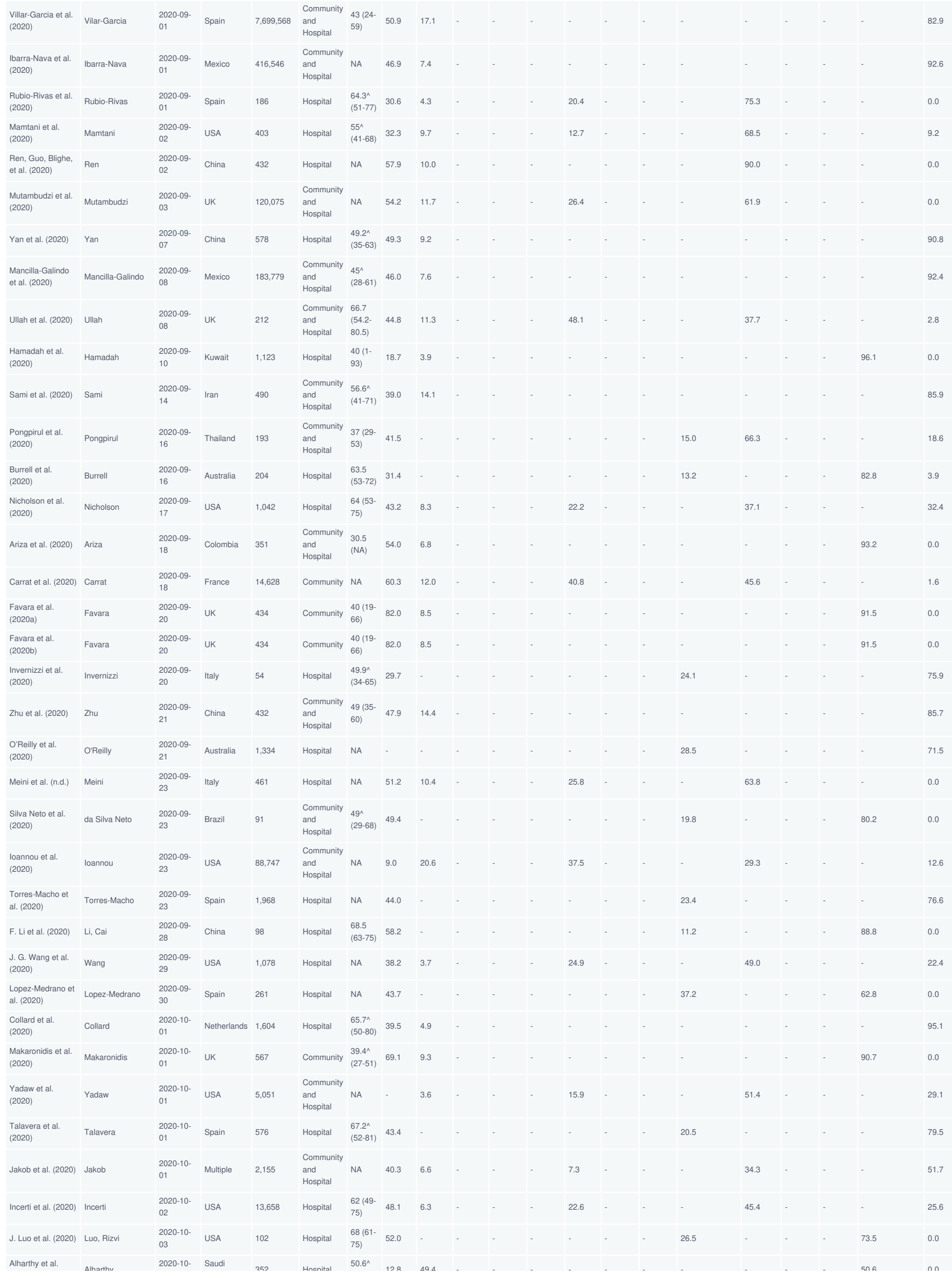




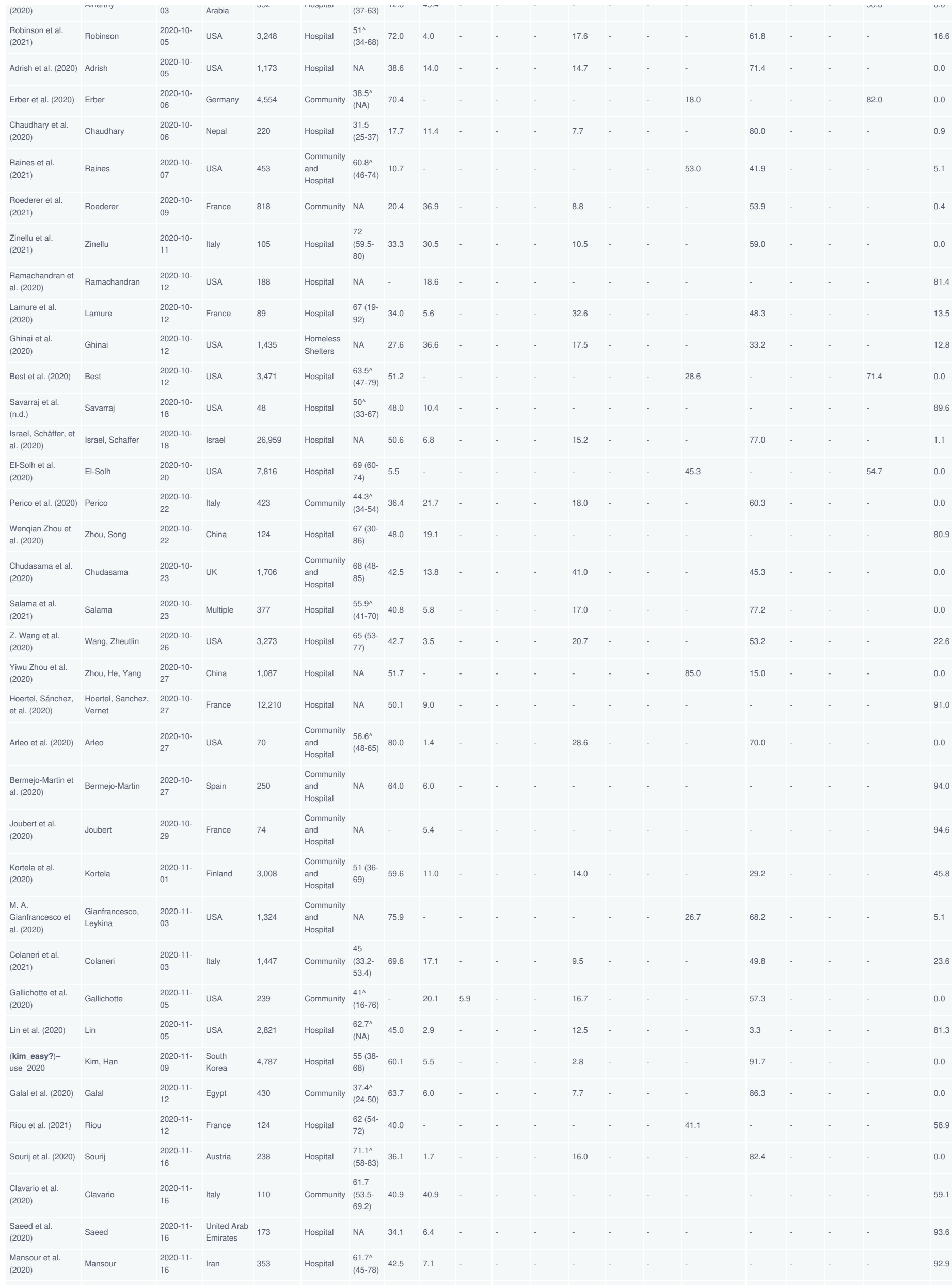




\begin{tabular}{|c|c|c|c|c|c|c|c|c|c|c|c|c|c|c|c|c|c|c|c|c|}
\hline $\begin{array}{l}\text { Cadegiani et al. } \\
\text { (2020) }\end{array}$ & Cadegiani & $\begin{array}{l}2020-11- \\
18\end{array}$ & Brazil & 130 & Community & $\begin{array}{l}42^{\wedge} \\
\text { (NA) }\end{array}$ & 0.0 & 7.7 & & & & - & - & & & & & & & 92.3 \\
\hline lic et al. (2021) & llic & $\begin{array}{l}2020-11- \\
19\end{array}$ & Serbia & 107 & $\begin{array}{l}\text { Community } \\
\text { and } \\
\text { Hospital }\end{array}$ & $\begin{array}{l}39.1^{\wedge} \\
(27-50)\end{array}$ & - & 29.9 & & & - & - & - & & & - & - & - & & 70.1 \\
\hline $\begin{array}{l}\text { Benaim et al. } \\
\text { (2020) }\end{array}$ & Benaim & $\begin{array}{l}2020-11- \\
19\end{array}$ & Israel & 693 & Hospital & $\begin{array}{l}59.8 \\
(\mathrm{NA})\end{array}$ & 47.9 & - & & & & - & - & & 5.1 & - & - & - & & 95.0 \\
\hline $\begin{array}{l}\text { F. K. Ho et al. } \\
\text { (2020) }\end{array}$ & Ho & $\begin{array}{l}2020-11- \\
19\end{array}$ & UK & 235,928 & $\begin{array}{l}\text { Community } \\
\text { and } \\
\text { Hospital }\end{array}$ & NA & & & & & & & & & 45.4 & & & & 54.6 & 0.0 \\
\hline Singh et al. (2020) & Singh & $\begin{array}{l}2020-11- \\
20\end{array}$ & UK & 930 & Hospital & $\begin{array}{l}71.4^{\wedge} \\
(54-87)\end{array}$ & 44.8 & & & & & & - & & 19.0 & & & & 81.0 & 0.0 \\
\hline $\begin{array}{l}\text { Márquez-Salinas } \\
\text { et al. (2020) }\end{array}$ & Marquez-Salinas & $\begin{array}{l}2020-11- \\
24\end{array}$ & Mexico & 1,068 & Hospital & $\begin{array}{l}53(44- \\
63)\end{array}$ & 36.8 & - & - & - & - & & & - & 15.0 & - & - & - & - & 85.0 \\
\hline $\begin{array}{l}\text { Díez-Manglano et } \\
\text { al. (2020) }\end{array}$ & Diez-Manglanas & $\begin{array}{l}2020-11- \\
24\end{array}$ & Spain & 4,393 & Hospital & $\begin{array}{l}53^{\wedge} \\
\text { (NA) }\end{array}$ & 40.8 & 6.6 & - & - & - & & & - & - & - & - & - & - & 93.4 \\
\hline Bellan et al. (2020) & Bellan & $\begin{array}{l}2020-11- \\
26\end{array}$ & Italy & 1,697 & Hospital & $\begin{array}{l}71(58- \\
80)\end{array}$ & 41.0 & 3.2 & - & - & - & - & & - & - & - & - & - & 15.2 & 81.6 \\
\hline $\begin{array}{l}\text { Woolcott \& } \\
\text { Castilla-Bancayán } \\
\text { (2021a) }\end{array}$ & Woolcott & $\begin{array}{l}2020-11- \\
26\end{array}$ & Mexico & $1,636,050$ & $\begin{array}{l}\text { Community } \\
\text { and } \\
\text { Hospital }\end{array}$ & $\begin{array}{l}42(34- \\
54)\end{array}$ & 51.9 & & & & & & & & 8.8 & & & & & 91.2 \\
\hline $\begin{array}{l}\text { Woolcott \& } \\
\text { Castilla-Bancayán } \\
\text { (2021b) }\end{array}$ & Woolcott & $\begin{array}{l}2020-11- \\
26\end{array}$ & Mexico & $1,636,050$ & $\begin{array}{l}\text { Community } \\
\text { and } \\
\text { Hospital }\end{array}$ & $\begin{array}{l}42(34- \\
54)\end{array}$ & 51.9 & - & & & & & - & & 8.8 & & & & & 91.2 \\
\hline $\begin{array}{l}\text { K. Yao et al. } \\
\text { (2021) }\end{array}$ & Yao, Hasegawa & $\begin{array}{l}2020-11- \\
26\end{array}$ & Japan & 101 & Hospital & $\begin{array}{l}60^{\wedge} \\
(17-97)\end{array}$ & 39.6 & - & - & - & - & - & & - & 28.7 & - & - & - & 71.3 & 0.0 \\
\hline $\begin{array}{l}\text { H. Chen et al. } \\
(2020)\end{array}$ & Chen, Varathraja & $\begin{array}{l}2020-11- \\
29\end{array}$ & USA & 10,123 & $\begin{array}{l}\text { Community } \\
\text { and } \\
\text { Hospital }\end{array}$ & $\begin{array}{l}40(28- \\
54)\end{array}$ & 53.6 & 4.4 & - & - & - & 9.7 & & - & - & 45.9 & - & - & - & 40.0 \\
\hline $\begin{array}{l}\text { Serling-Boyd et al. } \\
\text { (2020) }\end{array}$ & Serling-Boyd & $\begin{array}{l}2020-11- \\
30\end{array}$ & USA & 831 & Hospital & NA & 76.0 & 3.0 & & & & 23.2 & & & & 50.1 & & & & 23.7 \\
\hline $\begin{array}{l}\text { Simons et al. } \\
(2020)\end{array}$ & Simons & $\begin{array}{l}2020-11- \\
30\end{array}$ & UK & 446 & Hospital & $\begin{array}{l}64.9 \\
(52.4- \\
76.2)\end{array}$ & 35.9 & 9.4 & & & & 38.6 & - & & & 52.0 & & & & 0.0 \\
\hline $\begin{array}{l}\text { Dupraz et al. } \\
\text { (2020) }\end{array}$ & Dupraz & $\begin{array}{l}2020-11- \\
30\end{array}$ & Switzerland & 219 & Community & NA & 54.8 & 11.0 & & & & & - & & & & & & & 89.0 \\
\hline $\begin{array}{l}\text { Barasa et al. } \\
\text { (2020) }\end{array}$ & Barasa & $\begin{array}{l}2020-11- \\
30\end{array}$ & USA & 394 & Hospital & NA & 47.7 & 14.7 & & & & 36.0 & - & & & 41.6 & & - & & 7.6 \\
\hline $\begin{array}{l}\text { Ren, Guo, Tu, et } \\
\text { al. (2020) }\end{array}$ & Ren, Guo & $\begin{array}{l}2020-11- \\
30\end{array}$ & China & 481 & Hospital & NA & 45.7 & 7.7 & - & - & - & 0.6 & & - & - & 91.7 & - & - & - & 0.0 \\
\hline J. Li et al. (2020) & Li, Long, Zhang & $\begin{array}{l}2020-12- \\
03\end{array}$ & China & 954 & Hospital & NA & - & - & - & - & - & - & & - & 5.9 & 94.1 & - & - & - & 0.0 \\
\hline $\begin{array}{l}\text { Martini et al. } \\
(2020)\end{array}$ & Martini & $\begin{array}{l}\text { 2020-12- } \\
04\end{array}$ & Italy & 146 & Hospital & NA & 49.0 & - & - & & - & - & & & 46.6 & 53.4 & - & - & & 0.0 \\
\hline $\begin{array}{l}\text { O'Gallagher et al. } \\
\text { (2020) }\end{array}$ & O'Gallagher & $\begin{array}{l}2020-12- \\
04\end{array}$ & UK & 1,721 & Hospital & $\begin{array}{l}71(56- \\
83)\end{array}$ & 43.4 & 6.6 & & & & 18.5 & & & & 74.9 & & & & 0.0 \\
\hline $\begin{array}{l}\text { Alguwaihes et al. } \\
\text { (2020) }\end{array}$ & Alguwaihes & $\begin{array}{l}\text { 2020-12- } \\
05\end{array}$ & $\begin{array}{l}\text { Saudi } \\
\text { Arabia }\end{array}$ & 439 & Hospital & $\begin{array}{l}55(19- \\
101)\end{array}$ & 31.7 & 2.1 & & & & & & & & & & & 97.9 & 0.0 \\
\hline $\begin{array}{l}\text { Zuo, Warnock, et } \\
\text { al. (2020) }\end{array}$ & Zuo, Warnock & $\begin{array}{l}2020-12- \\
05\end{array}$ & USA & 118 & Hospital & $\begin{array}{l}61^{\wedge} \\
(44-78)\end{array}$ & 46.0 & & & & & & & & 23.7 & & & & & 76.3 \\
\hline $\begin{array}{l}\text { Xiaomeng Zhang } \\
\text { et al. (2020) }\end{array}$ & Zhang, Li & $\begin{array}{l}2020-12- \\
06\end{array}$ & UK & 1,746 & $\begin{array}{l}\text { Community } \\
\text { and } \\
\text { Hospital }\end{array}$ & $\begin{array}{l}68.8^{\wedge} \\
(59-78)\end{array}$ & 47.1 & 10.1 & & & & 35.1 & - & & & 44.2 & & & & 10.5 \\
\hline Dai et al. (2020) & Dai & $\begin{array}{l}\text { 2020-12- } \\
09\end{array}$ & China & 1,574 & Hospital & $\begin{array}{l}57.3^{\wedge} \\
(41-73)\end{array}$ & 48.2 & - & & & & - & - & & 9.2 & 90.8 & - & & & 0.0 \\
\hline $\begin{array}{l}\text { Vila-Córcoles et al. } \\
(2020)\end{array}$ & Vila-Corcoles & $\begin{array}{l}2020-12- \\
10\end{array}$ & Spain & 79,083 & Community & NA & 52.4 & - & - & - & - & - & & - & 16.1 & - & - & - & - & 83.9 \\
\hline Bisso et al. (2020) & Bisso & $\begin{array}{l}2020-12- \\
11\end{array}$ & Argentina & 168 & Hospital & $\begin{array}{l}67(58- \\
75)\end{array}$ & 34.0 & 10.7 & & - & - & - & & - & & - & - & - & & 89.3 \\
\hline $\begin{array}{l}\text { Christopher T. } \\
\text { Rentsch et al. } \\
\text { (2020a) }\end{array}$ & Rentsch, Beckman & $\begin{array}{l}2020-12- \\
11\end{array}$ & USA & 4,297 & Hospital & $\begin{array}{l}68(58- \\
75)\end{array}$ & 6.6 & 36.8 & & & & 39.3 & - & & & 1.9 & & & & 22.1 \\
\hline $\begin{array}{l}\text { Thiabaud et al. } \\
\text { (2020) }\end{array}$ & Thiabaud & $\begin{array}{l}2020-12- \\
11\end{array}$ & Switzerland & 3,582 & Hospital & $\begin{array}{l}68(54- \\
79)\end{array}$ & 40.5 & & & & & - & - & & 6.6 & & & & 50.5 & 42.9 \\
\hline $\begin{array}{l}\text { Vila-Corcoles et al. } \\
\text { (2020) }\end{array}$ & $\begin{array}{l}\text { Vila-Corcoles, } \\
\text { Satue-Gracia }\end{array}$ & $\begin{array}{l}2020-12- \\
11\end{array}$ & Spain & 282 & $\begin{array}{l}\text { Community } \\
\text { and } \\
\text { Hospital }\end{array}$ & $\begin{array}{l}65.9^{\wedge} \\
(53-78)\end{array}$ & 50.3 & 8.9 & - & - & - & - & & - & - & - & - & - & - & 91.1 \\
\hline Lévy et al. (2020) & Levy & $\begin{array}{l}2020-12- \\
12\end{array}$ & France & 61 & Hospital & $\begin{array}{l}60(50- \\
69)\end{array}$ & 20.0 & 8.2 & - & - & - & - & & - & - & - & - & - & - & 91.8 \\
\hline $\begin{array}{l}\text { Kantele et al. } \\
(2020)\end{array}$ & Kantele & $\begin{array}{l}2020-12- \\
13\end{array}$ & Finland & 1,095 & $\begin{array}{l}\text { Community } \\
\text { and } \\
\text { Hospital }\end{array}$ & $\begin{array}{l}38(31- \\
48)\end{array}$ & 82.7 & 16.0 & 0.2 & & & 25.3 & & & & 55.3 & & & & 0.0 \\
\hline Iftime et al. (2020) & Iftimie & $\begin{array}{l}2020-12- \\
14\end{array}$ & Spain & 468 & Hospital & NA & 44.9 & & & & & & - & & 7.9 & & & & & 92.1 \\
\hline $\begin{array}{l}\text { K. S. Ho et al. } \\
(2020)\end{array}$ & Ho, Narasimhan & $\begin{array}{l}2020-12- \\
15\end{array}$ & USA & 9,991 & $\begin{array}{l}\text { Community } \\
\text { and } \\
\text { Hospital }\end{array}$ & $\begin{array}{l}58^{\wedge} \\
(39-76)\end{array}$ & 45.9 & 4.1 & & & & 18.1 & - & & & 77.9 & & & & 0.0 \\
\hline $\begin{array}{l}\text { Caliskan \& Saylan } \\
(2020)\end{array}$ & Caliskan & $\begin{array}{l}2020-12- \\
16\end{array}$ & Turkey & 565 & Hospital & $\begin{array}{l}48(38- \\
58)\end{array}$ & - & 20.9 & & & & 14.0 & - & & & 65.1 & - & - & & 0.0 \\
\hline $\begin{array}{l}\text { Muñoz et al. } \\
\text { (2020) }\end{array}$ & Munoz & $\begin{array}{l}2020-12- \\
16\end{array}$ & Spain & 314 & Community & $\begin{array}{l}45(40- \\
53)\end{array}$ & 52.5 & 6.7 & & & & - & - & - & & - & - & - & & 93.3 \\
\hline $\begin{array}{l}\text { Crooks et al. } \\
(2020)\end{array}$ & Crooks & $\begin{array}{l}2020-12- \\
16\end{array}$ & UK & 2,964 & Hospital & NA & 52.6 & 11.7 & 5.1 & & & - & - & & & - & - & - & & 83.2 \\
\hline Nunez-Gil et al. & $\ldots$ & 2020-12- & & $\ldots$ & & 67 (53- & & -. & & & & & & & & & & & & ... \\
\hline
\end{tabular}




\begin{tabular}{|c|c|c|c|c|c|c|c|c|c|c|c|c|c|c|c|c|c|c|c|c|}
\hline (2020) & Nunez-Gill & 17 & Muttiple & 2,798 & Hospital & 78) & 40.0 & 6.2 & - & 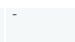 & $\cdot$ & & $\cdot$ & & & & & & & 93.8 \\
\hline $\begin{array}{l}\text { Núñez-Gil et al. } \\
\text { (2021) }\end{array}$ & Nunez-Gil & $\begin{array}{l}2020-12- \\
17\end{array}$ & Multiple & 2,798 & Hospital & $\begin{array}{l}67(53- \\
78)\end{array}$ & 40.0 & 6.2 & & & & & & & & & & & & 93.8 \\
\hline Gori et al. (2020) & Gori & $\begin{array}{l}2020-12- \\
17\end{array}$ & Italy & 1,352 & Hospital & $\begin{array}{l}68(58- \\
77)\end{array}$ & 28.4 & 3.6 & & & & 18.4 & & & & 66.6 & & & & 11.4 \\
\hline $\begin{array}{l}\text { Rowlands et al. } \\
\text { (2020) }\end{array}$ & Rowlands & $\begin{array}{l}2020-12- \\
18\end{array}$ & UK & 580 & Community & $\begin{array}{l}63.8^{\wedge} \\
(56-70)\end{array}$ & 52.2 & 10.2 & & & & 38.1 & & & & 51.7 & & & & 0.0 \\
\hline 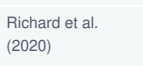 & Richard & $\begin{array}{l}2020-12- \\
18\end{array}$ & Switzerland & 8,344 & Community & $\begin{array}{l}46.99 \\
\text { (NA) }\end{array}$ & 53.5 & 15.2 & - & - & - & 17.1 & - & & & 66.2 & & & & 1.5 \\
\hline $\begin{array}{l}\text { Schubl et al. } \\
\text { (2020) }\end{array}$ & Schuble ${ }^{*}$ & $\begin{array}{l}2020-12- \\
19\end{array}$ & USA & 1,557 & Community & NA & 68.9 & 2.4 & & & & & & & & & & & & 97.6 \\
\hline $\begin{array}{l}\text { Ugur Chousein et } \\
\text { al. (2020) }\end{array}$ & Chousein & $\begin{array}{l}2020-12- \\
21\end{array}$ & Turkey & 114 & Hospital & $\begin{array}{l}51.1^{\wedge} \\
(36-66)\end{array}$ & 32.5 & 16.7 & & & & 20.2 & & & & 63.2 & & & & 0.0 \\
\hline $\begin{array}{l}\text { Modrák et al. } \\
(2020)\end{array}$ & Modrak & $\begin{array}{l}2020-12- \\
22\end{array}$ & Czechia & 213 & Hospital & $\begin{array}{l}69(58- \\
80)\end{array}$ & 51.0 & & - & & - & & - & - & 13.1 & & & & & 86.8 \\
\hline $\begin{array}{l}\text { Kara Polat et al. } \\
(2020)\end{array}$ & Polat & $\begin{array}{l}2020-12- \\
22\end{array}$ & Turkey & 1,322 & Community & NA & 47.6 & 35.6 & - & & - & 15.6 & - & - & & 48.8 & & & & 0.0 \\
\hline $\begin{array}{l}\text { Kjettand et al. } \\
(2020)\end{array}$ & Kjettand & $\begin{array}{l}2020-12- \\
24\end{array}$ & Norway & 7,839 & $\begin{array}{l}\text { Community } \\
\text { and } \\
\text { Hospital }\end{array}$ & $\begin{array}{l}45.33^{1} \\
(33-56)\end{array}$ & 77.0 & & & & & & & & 41.0 & & & & & 59.0 \\
\hline $\begin{array}{l}\text { Lewnard et al. } \\
\text { (2021) }\end{array}$ & Lewnard & $\begin{array}{l}2021-01- \\
02\end{array}$ & USA & 1,115 & Community & NA & 52.6 & 4.4 & & & & 14.2 & & & & 81.3 & & & & 0.1 \\
\hline $\begin{array}{l}\text { Nezzhadmoghadam } \\
\text { \& Tamez-Peña } \\
\text { (2021) }\end{array}$ & Nezhadmoghadam & $\begin{array}{l}\text { 2021-01- } \\
04\end{array}$ & Mexico & 33,325 & Hospital & NA & & 10.2 & & & & & & & & & & & & 89.8 \\
\hline $\begin{array}{l}\text { Covid-19 in } \\
\text { pregnancy et al. } \\
(2021)\end{array}$ & Vousden & $\begin{array}{l}\text { 2021-01- } \\
05\end{array}$ & UK & 1,148 & $\begin{array}{l}\text { Community } \\
\text { and } \\
\text { Hospital }\end{array}$ & NA & 100.0 & 8.6 & & & & & & & & & & & & 91.4 \\
\hline $\begin{array}{l}\text { Boyd \& Martin- } \\
\text { Loeches (2021) }\end{array}$ & Boyd & $\begin{array}{l}2021-01- \\
07\end{array}$ & Ireland & 38 & Hospital & NA & 26.3 & & & & & & & & 5.3 & & & & & 94.7 \\
\hline $\begin{array}{l}\text { Paleiron et al. } \\
\text { (2021) }\end{array}$ & Paleiron & $\begin{array}{l}2021-01- \\
09\end{array}$ & France & 1,688 & Community & $\begin{array}{l}28(23- \\
35)\end{array}$ & 13.0 & 42.1 & 12.9 & - & - & 19.9 & - & & & 25.1 & & & & 0.0 \\
\hline Ader et al. (2021) & Ader & $\begin{array}{l}2021-01- \\
09\end{array}$ & Multiple & 583 & Hospital & $\begin{array}{l}63(54- \\
71)\end{array}$ & 28.3 & 3.1 & & & & & & & & & & & & 96.9 \\
\hline $\begin{array}{l}\text { Giannini et al. } \\
(2021)\end{array}$ & Giannini & $\begin{array}{l}2021-01- \\
10\end{array}$ & Italy & 91 & Hospital & $\begin{array}{l}74 \wedge \\
(61-87)\end{array}$ & 45.0 & 23.1 & & & & & & & & & & & & 76.9 \\
\hline $\begin{array}{l}\text { Shade et al. } \\
\text { (2021) }\end{array}$ & Shade & $\begin{array}{l}2021-01- \\
10\end{array}$ & USA & 3,779 & $\begin{array}{l}\text { Community } \\
\text { and } \\
\text { Hospital }\end{array}$ & NA & 47.2 & 3.3 & - & - & - & 15.7 & - & & & & & & & 80.9 \\
\hline Park et al. (2021) & Park & $\begin{array}{l}2021-01- \\
11\end{array}$ & $\begin{array}{l}\text { South } \\
\text { Korea }\end{array}$ & 2,269 & Hospital & $\begin{array}{l}55.5^{\wedge} \\
(35-75)\end{array}$ & 64.1 & 4.1 & & & & & & & & & & & & 95.9 \\
\hline $\begin{array}{l}\text { Ferrari et al. } \\
\text { (2021) }\end{array}$ & Ferrari & $\begin{array}{l}2021-01- \\
12\end{array}$ & Brazil & 198 & Community & $\begin{array}{l}61(19- \\
91)\end{array}$ & 65.0 & & & & & & & & 20.7 & 79.3 & & & & 0.0 \\
\hline $\begin{array}{l}\text { Y. Zhang et al. } \\
\text { (2021) }\end{array}$ & Zhang, Yang & $\begin{array}{l}2021-01- \\
12\end{array}$ & UK & 1,485 & $\begin{array}{l}\text { Community } \\
\text { and } \\
\text { Hospital }\end{array}$ & $\begin{array}{l}68.2^{\wedge} \\
(59-77)\end{array}$ & 47.2 & 48.5 & & & & 39.2 & & & & 11.3 & & & & 1.0 \\
\hline $\begin{array}{l}\text { Ebrahimian et al. } \\
(2021)\end{array}$ & Ebrahimian & $\begin{array}{l}2021-01- \\
13\end{array}$ & USA & 226 & Hospital & NA & & & & & & & & & 10.6 & & & & 89.4 & 0.0 \\
\hline $\begin{array}{l}\text { Vahidy et al. } \\
\text { (2021) }\end{array}$ & Vahidy & $\begin{array}{l}2021-01- \\
13\end{array}$ & USA & 96,473 & Hospital & $\begin{array}{l}51.2^{\wedge} \\
(32-69)\end{array}$ & 59.6 & & & & & & & & 26.2 & 73.8 & & & & 0.0 \\
\hline $\begin{array}{l}\text { Mendes et al. } \\
(2021)\end{array}$ & Mendes & $\begin{array}{l}2021-01- \\
14\end{array}$ & Switzerland & 265 & Hospital & $\begin{array}{l}85.9^{1} \\
(79-92)\end{array}$ & 57.0 & 5.7 & & & & & & & & & & & & 94.3 \\
\hline $\begin{array}{l}\text { Saurabh et al. } \\
\text { (2021) }\end{array}$ & Saurabh & $\begin{array}{l}2021-01- \\
14\end{array}$ & India & 911 & Community & $\begin{array}{l}\begin{array}{l}43.1^{\wedge} \\
(23-62)\end{array} \\
(25)\end{array}$ & 35.2 & 7.0 & & & & 0.6 & & & & 79.1 & & & & 0.0 \\
\hline $\begin{array}{l}\text { Ayoubkhani et al. } \\
\text { (2021) }\end{array}$ & Ayoubkhani & $\begin{array}{l}2021-01- \\
15\end{array}$ & UK & 47,780 & Hospital & NA & 45.1 & 8.4 & & & & 40.9 & & & & 42.5 & & & & 8.2 \\
\hline $\begin{array}{l}\text { Thakur et al. } \\
\text { (2021) }\end{array}$ & Thakar & $\begin{array}{l}2021-01- \\
18\end{array}$ & India & 250 & Hospital & NA & 42.4 & & & & & & & & 49.2 & & & & 50.8 & 0.0 \\
\hline Zhong et al. (2020) & Zhong & $\begin{array}{l}2021-01- \\
18\end{array}$ & China & 91 & Hospital & $\begin{array}{l}47.3^{n} \\
(30-64)\end{array}$ & 49.5 & & & & & & & & 18.7 & & & & & 81.3 \\
\hline $\begin{array}{l}\text { Cummins et al. } \\
\text { (2021) }\end{array}$ & Cummins & $\begin{array}{l}2021-01- \\
20\end{array}$ & UK & 1,781 & $\begin{array}{l}\text { Community } \\
\text { and } \\
\text { Hospital }\end{array}$ & $\mathrm{NA}$ & 44.8 & 10.2 & & & & & & & & & & & & 89.8 \\
\hline Sun et al. (2021) & Sun & $\begin{array}{l}\text { 2021-01- } \\
21\end{array}$ & USA & 323 & $\begin{array}{l}\text { Community } \\
\text { and } \\
\text { Hospital }\end{array}$ & NA & 57.6 & & & & & & & & 39.3 & & & & 60.7 & 0.0 \\
\hline Lowe et al. (2021) & Lowe & $\begin{array}{l}2021-01- \\
25\end{array}$ & USA & 7,102 & Hospital & $\begin{array}{l}503^{1} \\
(\mathrm{NA})\end{array}$ & 61.2 & 2.4 & & & & 12.8 & & & & 84.8 & & & & 0.0 \\
\hline $\begin{array}{l}\text { Caglar \& Kacer } \\
\text { (2021) }\end{array}$ & Caglar & $\begin{array}{l}2021-01- \\
25\end{array}$ & Turkey & 120 & Hospital & $\begin{array}{l}57(47- \\
67)\end{array}$ & 51.7 & & & & & & & & 37.5 & & & & 62.5 & 0.0 \\
\hline $\begin{array}{l}\text { De Santi et al. } \\
\text { (2021) }\end{array}$ & De, Santi & $\begin{array}{l}2021-01- \\
25\end{array}$ & Italy & 341 & Community & $\begin{array}{l}47(20- \\
82)\end{array}$ & 51.6 & 25.8 & & & & & & & & & & & & 74.2 \\
\hline Quan et al. (2021) & Quan & $\begin{array}{l}2021-01- \\
27\end{array}$ & USA & 2,038 & Hospital & $\begin{array}{l}64 \wedge \\
(47-80)\end{array}$ & 50.4 & & & & & & & & 39.7 & & & & & 60.3 \\
\hline $\begin{array}{l}\text { Strangfeld et al. } \\
\text { (2021) }\end{array}$ & Strangfeld & $\begin{array}{l}2021-01- \\
27\end{array}$ & Multiple & 3,729 & $\begin{array}{l}\text { Community } \\
\text { and } \\
\text { Hospital }\end{array}$ & $\begin{array}{l}57 \wedge \\
(41-72)\end{array}$ & 68.0 & & & & & & & & 20.8 & 64.0 & & & & 15.2 \\
\hline Tardif et al. (2021) & Tardif & $\begin{array}{l}2021-01- \\
27\end{array}$ & Canada & 4,488 & $\begin{array}{l}\text { Community } \\
\text { and } \\
\text { Hospital }\end{array}$ & NA & 53.9 & 9.6 & - & - & - & 33.6 & - & & & 56.8 & & & & 0.0 \\
\hline Jafari et al. (2021) & Jafari & $\begin{array}{l}2021-01- \\
28\end{array}$ & USA & 1,979 & Hospital & $\begin{array}{l}66(57- \\
74)\end{array}$ & 32.0 & 12.1 & & & & & & & & & & & & 87.9 \\
\hline
\end{tabular}




\begin{tabular}{|c|c|c|c|c|c|c|c|c|}
\hline $\begin{array}{l}\text { Tobed, et al. } \\
\text { (2021) }\end{array}$ & Prats-Uribe & $\begin{array}{l}20<1-U 1- \\
30\end{array}$ & Spain & 696 & Hospital & $\begin{array}{l}63^{n} \\
(52-73)\end{array}$ & 30.9 & - \\
\hline Nuño et al. (2021) & Nuno & $\begin{array}{l}2021-02- \\
01\end{array}$ & USA & 4,730 & Hospital & $\begin{array}{l}61(46- \\
73)\end{array}$ & 43.6 & 10 \\
\hline Abajo et al. (2021) & de Abajo & $\begin{array}{l}2021-02- \\
03\end{array}$ & Spain & 625 & Hospital & NA & 39.4 & 4.6 \\
\hline Mora et al. (2021) & Mora & $\begin{array}{l}2021-02- \\
03\end{array}$ & USA & 1,058 & Community & $\begin{array}{l}39.7^{\wedge} \\
(27-52)\end{array}$ & 52.5 & 4.5 \\
\hline $\begin{array}{l}\text { Molenaar et al. } \\
\text { (2021) }\end{array}$ & Molenaar & $\begin{array}{l}2021-02- \\
03\end{array}$ & USA & 696 & $\begin{array}{l}\text { Community } \\
\text { and } \\
\text { Hospital }\end{array}$ & $\begin{array}{l}33.1^{\wedge} \\
(\mathrm{NA})\end{array}$ & 100.0 & 4.5 \\
\hline $\begin{array}{l}\text { Leister et al. } \\
\text { (2021) }\end{array}$ & Leister & $\begin{array}{l}2021-02- \\
03\end{array}$ & Austria & 3,301 & $\begin{array}{l}\text { Community } \\
\text { and } \\
\text { Hospital }\end{array}$ & $\begin{array}{l}43.6^{\wedge} \\
(33-54)\end{array}$ & 68.0 & 23 \\
\hline $\begin{array}{l}\text { Didikoglu et al. } \\
\text { (2021) }\end{array}$ & Didikoglu & $\begin{array}{l}2021-02- \\
04\end{array}$ & UK & 384,816 & $\begin{array}{l}\text { Community } \\
\text { and } \\
\text { Hospital }\end{array}$ & $\begin{array}{l}68.3^{\wedge} \\
(60-76)\end{array}$ & 54.9 & 9.8 \\
\hline Estiri et al. (2021) & Estiri & $\begin{array}{l}2021-02- \\
04\end{array}$ & USA & 16,709 & Hospital & NA & 57.2 & \\
\hline $\begin{array}{l}\text { Tavakol et al. } \\
\text { (2021) }\end{array}$ & Tavakol & $\begin{array}{l}2021-02- \\
04\end{array}$ & Iran & 206 & $\begin{array}{l}\text { Community } \\
\text { and } \\
\text { Hospital }\end{array}$ & $\begin{array}{l}40.9^{\wedge} \\
(29-52)\end{array}$ & 57.8 & \\
\hline Cai et al. (2021) & Cai, Yang & $\begin{array}{l}2021-02- \\
05\end{array}$ & China & 455 & Hospital & NA & 52.6 & 7.5 \\
\hline Lohia et al. (2021) & Lohia & $\begin{array}{l}2021-02- \\
05\end{array}$ & USA & 1,871 & Hospital & $\begin{array}{l}64.1^{\wedge} \\
(48-80)\end{array}$ & 48.4 & \\
\hline $\begin{array}{l}\text { Vila-Corcoles et al. } \\
\text { (2021) }\end{array}$ & $\begin{array}{l}\text { Vila-Corcoles, } \\
\text { Satue-Gracia, Vila- } \\
\text { Rovira }\end{array}$ & $\begin{array}{l}2021-02- \\
05\end{array}$ & Spain & 79,083 & Community & $\begin{array}{l}65.8^{\wedge} \\
(54-77)\end{array}$ & 52.4 & 16. \\
\hline $\begin{array}{l}\text { Gégout petit et al. } \\
\text { (2021) }\end{array}$ & Gegout Petit & $\begin{array}{l}2021-02- \\
12\end{array}$ & France & 2,006 & Community & NA & 55.0 & 16.8 \\
\hline $\begin{array}{l}\text { Q. Zhang et al. } \\
\text { (2021) }\end{array}$ & Zhang, Wang & $\begin{array}{l}2021-02- \\
13\end{array}$ & China & 172 & Hospital & $\begin{array}{l}47.9^{\wedge} \\
(29-66)\end{array}$ & 46.5 & 7.0 \\
\hline $\begin{array}{l}\text { Dambha-Miller et } \\
\text { al. (2021) }\end{array}$ & Dambha, Miller & $\begin{array}{l}2021-02- \\
19\end{array}$ & UK & 5,451 & $\begin{array}{l}\text { Community } \\
\text { and } \\
\text { Hospital }\end{array}$ & $\begin{array}{l}59^{\wedge} \\
(37-80)\end{array}$ & 100.0 & 8.9 \\
\hline Badr et al. (2021a) & Ibrahim, Badr & $\begin{array}{l}2021-02- \\
19\end{array}$ & $\begin{array}{l}\text { Saudi } \\
\text { Arabia }\end{array}$ & 159 & Hospital & NA & 30.0 & \\
\hline Badr et al. (2021b) & Ibrahim, Badr & $\begin{array}{l}2021-02- \\
19\end{array}$ & $\begin{array}{l}\text { Saudi } \\
\text { Arabia }\end{array}$ & 159 & Hospital & NA & 30.0 & \\
\hline $\begin{array}{l}\text { Hoertel et al. } \\
\text { (2021) }\end{array}$ & Hoertel, A & $\begin{array}{l}2021-02- \\
20\end{array}$ & France & 545 & $\begin{array}{l}\text { Community } \\
\text { and } \\
\text { Hospital }\end{array}$ & NA & 46.0 & 21 \\
\hline $\begin{array}{l}\text { Lombardi et al. } \\
\text { (2021) }\end{array}$ & Lombardi & $\begin{array}{l}2021-02- \\
22\end{array}$ & Italy & 4,055 & Community & $\begin{array}{l}44.8^{\wedge} \\
(\mathrm{NA})\end{array}$ & 69.6 & 2 \\
\hline $\begin{array}{l}\text { Gharebaghi et al. } \\
\text { (2021) }\end{array}$ & Gharebaghi & $\begin{array}{l}2021-02- \\
24\end{array}$ & Iran & 215 & Hospital & $\begin{array}{l}50.9^{\wedge} \\
(33-68)\end{array}$ & 42.3 & 20.5 \\
\hline $\begin{array}{l}\text { Shields et al. } \\
\text { (2021) }\end{array}$ & Shields & $\begin{array}{l}2021-02- \\
26\end{array}$ & UK & 1,507 & Community & $\begin{array}{l}37(29- \\
47)\end{array}$ & 75.4 & 1( \\
\hline Voruz et al. (2021) & Voruz & $\begin{array}{l}2021-02- \\
26\end{array}$ & Switzerland & 45 & $\begin{array}{l}\text { Community } \\
\text { and } \\
\text { Hospital }\end{array}$ & NA & 42.0 & \\
\hline $\begin{array}{l}\text { The OpenSAFELY } \\
\text { Collaborative et al. } \\
\text { (2021) }\end{array}$ & $\begin{array}{l}\text { Williamson, } \\
\text { Tazare }\end{array}$ & $\begin{array}{l}2021-03- \\
01\end{array}$ & UK & $11,972,947$ & Community & NA & 52.1 & $1 \xi$ \\
\hline $\begin{array}{l}\text { González, Vielot, } \\
\text { et al. (2021) }\end{array}$ & Gonzalez, Vielot & $\begin{array}{l}2021-03- \\
01\end{array}$ & Nicaragua & 1,351 & Community & NA & 59.0 & \\
\hline Lucar et al. (2021) & Lucar & $\begin{array}{l}2021-03- \\
01\end{array}$ & USA & 100 & Hospital & $\begin{array}{l}59(44- \\
70)\end{array}$ & 53.0 & \\
\hline Drozd et al. (2021) & Drozd & $\begin{array}{l}2021-03- \\
01\end{array}$ & UK & 493,295 & Community & NA & 54.5 & 10.6 \\
\hline $\begin{array}{l}\text { Printza et al. } \\
\text { (2021) }\end{array}$ & Printza & $\begin{array}{l}2021-03- \\
02\end{array}$ & Greece & 150 & Hospital & $\begin{array}{l}51.6^{\wedge} \\
(34-68)\end{array}$ & 38.0 & 1 \\
\hline Dashti et al. (2021) & Dashti & $\begin{array}{l}2021-03- \\
02\end{array}$ & USA & 12,347 & Hospital & $\begin{array}{l}47(32- \\
62)\end{array}$ & 53.3 & 4.6 \\
\hline $\begin{array}{l}\text { Nielsen et al. } \\
\text { (2021) }\end{array}$ & ielsen & $\begin{array}{l}2021-03- \\
03\end{array}$ & Denmark & 840 & Community & NA & 84.0 & 4.6 \\
\hline Peng et al. (2021) & Peng, Lei & $\begin{array}{l}2021-03- \\
03\end{array}$ & China & 622 & Hospital & NA & 48.9 & 1 \\
\hline $\begin{array}{l}\text { Dayem Ullah et al. } \\
\text { (2021) }\end{array}$ & Dayem, Ullah & $\begin{array}{l}2021-03- \\
04\end{array}$ & UK & 15,440 & Community & $\begin{array}{l}57.2 \\
(44.9- \\
69.4)\end{array}$ & 56.2 & 21 \\
\hline $\begin{array}{l}\text { Velasco- } \\
\text { Rodríguez et al. } \\
\text { (2021) }\end{array}$ & $\begin{array}{l}\text { Velasco, } \\
\text { Rodriguez }\end{array}$ & $\begin{array}{l}2021-03- \\
04\end{array}$ & Spain & 2,070 & Hospital & $\begin{array}{l}67(54- \\
79)\end{array}$ & 42.7 & 4.1 \\
\hline $\begin{array}{l}\text { Action to beat } \\
\text { coronavirus/Action } \\
\text { pour battre le } \\
\text { coronavirus (Ab-C) } \\
\text { Study } \\
\text { Investigators \& } \\
\text { Jha (2021) }\end{array}$ & Jha & $\begin{array}{l}2021-03- \\
05\end{array}$ & Canada & 8,967 & Community & NA & 57.0 & - \\
\hline $\begin{array}{l}\text { Guzmán et al. } \\
\text { (2021) }\end{array}$ & Macias, Guzman & $\begin{array}{l}2021-03- \\
08\end{array}$ & Mexico & 196 & Hospital & $\begin{array}{l}58.1^{\wedge} \\
(42-73)\end{array}$ & 62.0 & \\
\hline & & 20 & & & & & & \\
\hline
\end{tabular}




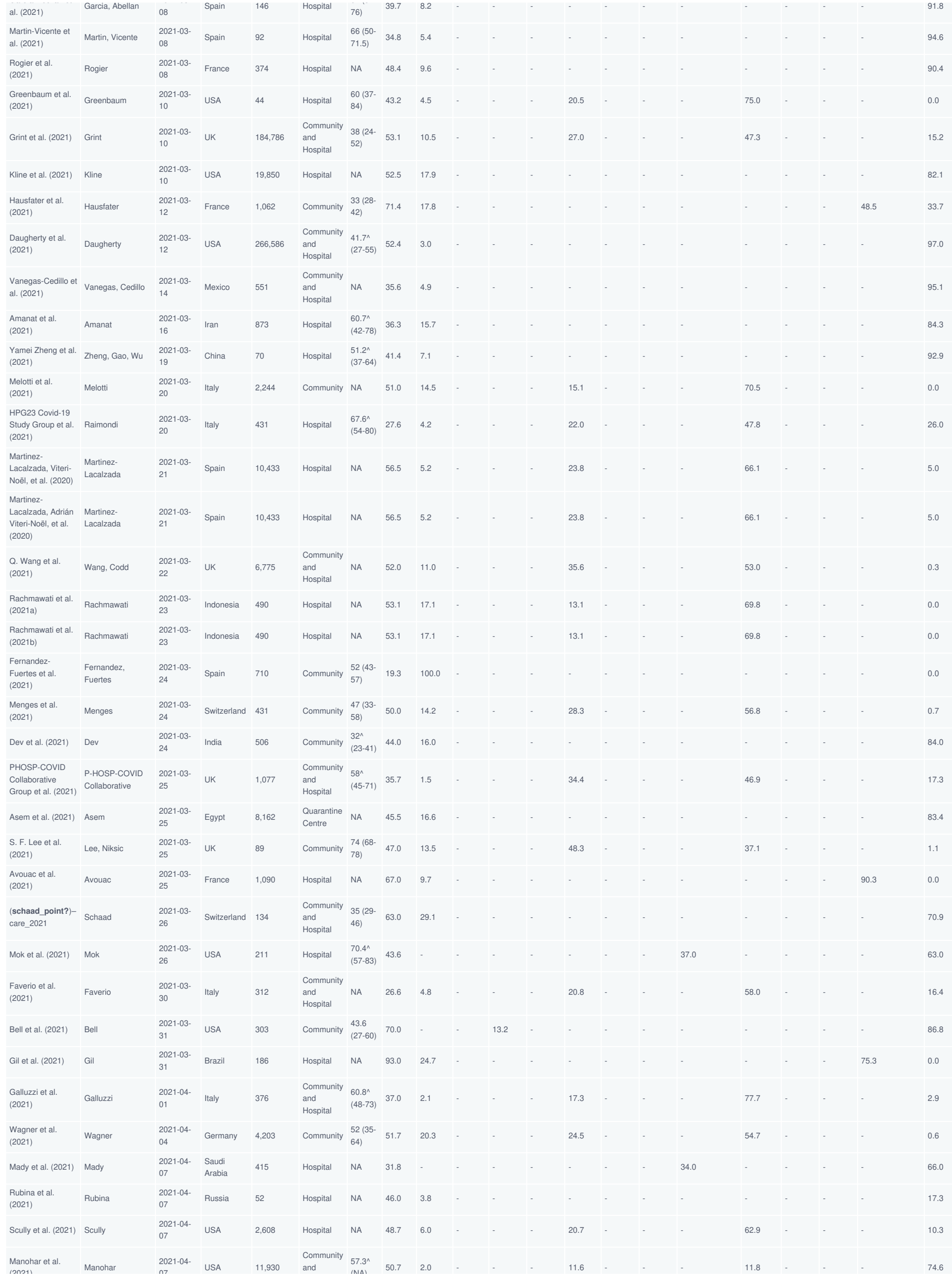




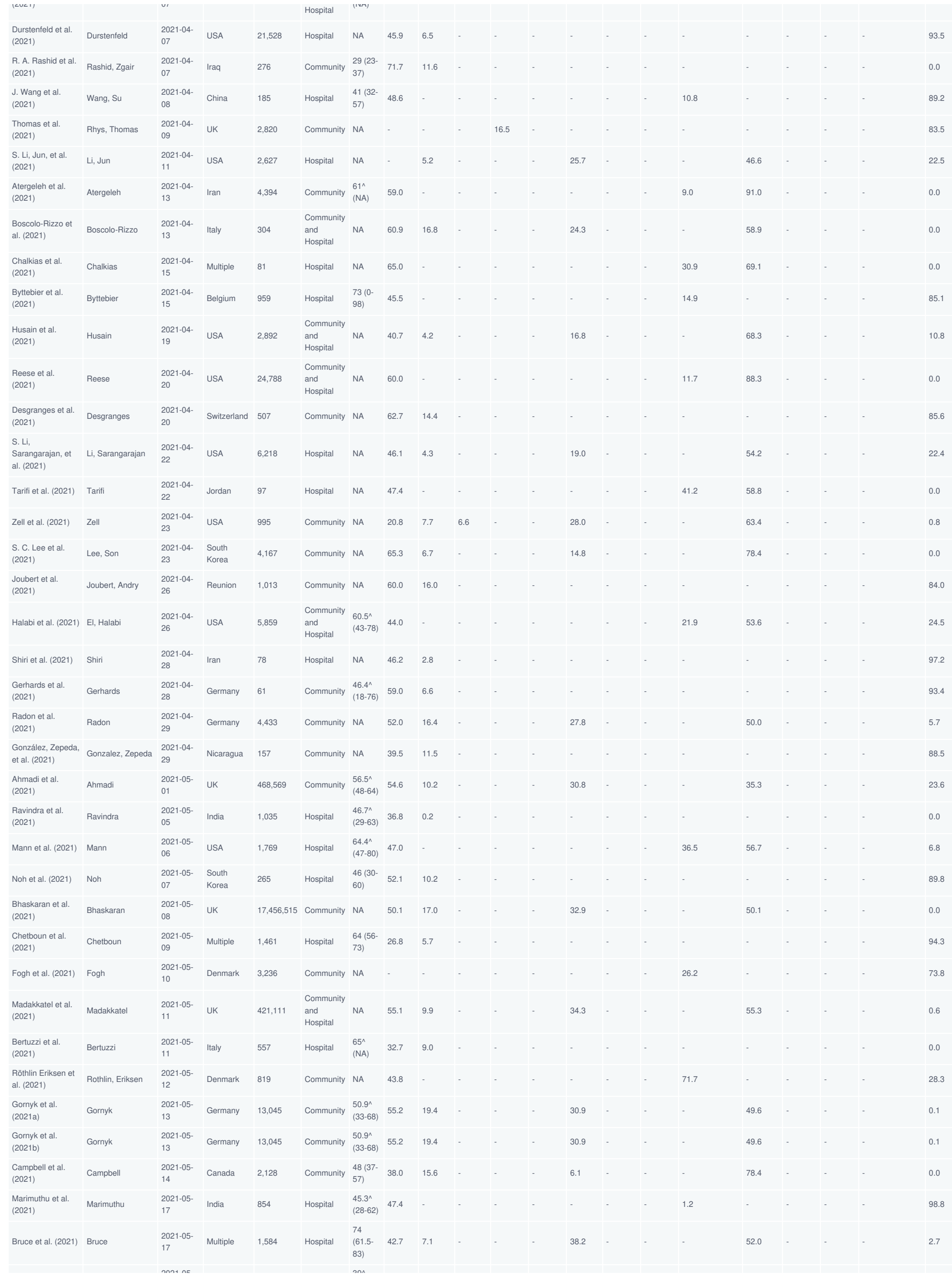




\begin{tabular}{|c|c|c|c|c|c|c|c|c|c|c|c|c|c|c|c|c|c|c|c|c|}
\hline Bark et al. (2021) & Bark & $\begin{array}{l}\text { cu<i-uj- } \\
18\end{array}$ & Canada & 157 & Community & $\begin{array}{l}(18-73) \\
\text { (18) }\end{array}$ & 66.0 & - & & 17.2 & - & - & - & - & - & - & - & & - & 82.8 \\
\hline Jha et al. (2021) & Jha, Shrestha & $\begin{array}{l}2021-05- \\
18\end{array}$ & Nepal & 198 & Hospital & $\begin{array}{l}40.8 \\
(29.8- \\
51.3)\end{array}$ & 26.3 & 11.6 & & & - & 12.6 & - & - & - & 75.8 & - & & - & 0.0 \\
\hline $\begin{array}{l}\text { Lekoubou et al. } \\
\text { (2021) }\end{array}$ & Lekoubou & $\begin{array}{l}2021-05- \\
18\end{array}$ & USA & 8,815 & Hospital & $\begin{array}{l}51^{\wedge} \\
(33-69)\end{array}$ & 57.0 & 2.9 & - & - & - & 6.6 & - & - & - & 18.1 & - & & - & 72.3 \\
\hline $\begin{array}{l}\text { Kahlert et al. } \\
\text { (2021) }\end{array}$ & Kahlert & $\begin{array}{l}2021-05- \\
18\end{array}$ & Switzerland & 4,664 & Community & $\begin{array}{l}38.3 \\
(29.7- \\
49.5)\end{array}$ & 78.3 & 17.6 & - & & & 20.4 & & & & 62.0 & & - & & 0.0 \\
\hline $\begin{array}{l}\text { Hirschtick et al. } \\
\text { (2021) }\end{array}$ & Hirschtick & $\begin{array}{l}2021-05- \\
19\end{array}$ & USA & 593 & Community & $\begin{array}{l}51.5^{\wedge} \\
(35-67)\end{array}$ & 56.0 & 6.2 & - & & & & & & & & & - & & 93.8 \\
\hline Y. Li et al. (2021) & Li, Tong & $\begin{array}{l}2021-05- \\
19\end{array}$ & USA & 18,148 & Community & $\begin{array}{l}47(37- \\
56)\end{array}$ & 67.1 & 12.3 & - & & & & & & & & & - & & 0.1 \\
\hline $\begin{array}{l}\text { AlSafar et al. } \\
\text { (2021) }\end{array}$ & Alsatar & $\begin{array}{l}2021-05- \\
19\end{array}$ & $\begin{array}{l}\text { United Arab } \\
\text { Emirates }\end{array}$ & 464 & $\begin{array}{l}\text { Community } \\
\text { and } \\
\text { Hospital }\end{array}$ & $\begin{array}{l}47^{\wedge} \\
(32-62)\end{array}$ & 40.0 & 10.8 & - & & & & & - & & & & - & 89.2 & 0.0 \\
\hline Wallis et al. (2021) & Wallis & $\begin{array}{l}2021-05- \\
21\end{array}$ & UK & 101 & Hospital & $\begin{array}{l}53(45- \\
63)\end{array}$ & 46.5 & - & - & - & - & & & - & 34.7 & & & - & & 65.3 \\
\hline Ayhan et al. (2021) & Ayhan & $\begin{array}{l}2021-05- \\
21\end{array}$ & Turkey & 92 & Hospital & $\begin{array}{l}67(55- \\
73)\end{array}$ & 44.6 & 17.4 & - & - & - & & & - & & & & - & 43.5 & 39.1 \\
\hline $\begin{array}{l}\text { Nanda et al. } \\
\text { (2021) }\end{array}$ & Nanda & $\begin{array}{l}2021-05- \\
24\end{array}$ & USA & 1,169 & Hospital & $\begin{array}{l}28.6 \\
\text { (NA) }\end{array}$ & 50.7 & 12.8 & - & - & - & 14.9 & & - & & 72.3 & & - & & 0.0 \\
\hline $\begin{array}{l}\text { Prats-Uribe, Xie, et } \\
\text { al. (2021) }\end{array}$ & Prats-Uribe, Xie & $\begin{array}{l}2021-05- \\
25\end{array}$ & UK & 1,591 & $\begin{array}{l}\text { Community } \\
\text { and } \\
\text { Hospital }\end{array}$ & NA & 47.5 & 12.1 & - & - & - & 39.5 & & - & - & 48.5 & & - & & 0.0 \\
\hline $\begin{array}{l}\text { Egede et al. } \\
\text { (2021) }\end{array}$ & Egede & $\begin{array}{l}2021-05- \\
26\end{array}$ & USA & 30,976 & $\begin{array}{l}\text { Community } \\
\text { and } \\
\text { Hospital }\end{array}$ & $\begin{array}{l}52.8^{\wedge} \\
(33-71)\end{array}$ & 60.0 & 13.9 & & & & 32.3 & & & & & & - & 53.8 & 0.0 \\
\hline $\begin{array}{l}\text { Kleynhans et al. } \\
\text { (2021) }\end{array}$ & Kleynhans & $\begin{array}{l}2021-05- \\
29\end{array}$ & South Africa & 350 & Community & NA & 65.0 & 10.3 & - & & & & & & & & & - & & 35.4 \\
\hline $\begin{array}{l}\text { Puebla Neira et al. } \\
\text { (2021) }\end{array}$ & Puebla, Neira & $\begin{array}{l}2021-06- \\
01\end{array}$ & USA & 10,216 & $\begin{array}{l}\text { Community } \\
\text { and } \\
\text { Hospital }\end{array}$ & $\begin{array}{l}45.8^{\wedge} \\
(27-63)\end{array}$ & 64.9 & 3.9 & - & - & & 9.0 & & - & & 87.1 & & - & & 0.0 \\
\hline $\begin{array}{l}\text { Lassen et al. } \\
\text { (2021) }\end{array}$ & Hojbjerg, Lassen & $\begin{array}{l}2021-06- \\
01\end{array}$ & Denmark & 171 & Hospital & NA & 55.0 & 5.8 & - & - & - & 53.8 & & - & & 38.6 & & - & & 1.8 \\
\hline $\begin{array}{l}\text { S. Chen et al. } \\
\text { (2021) }\end{array}$ & Chen, Zheng & $\begin{array}{l}2021-06- \\
06\end{array}$ & China & 1,030 & Hospital & $\begin{array}{l}55(44- \\
65)\end{array}$ & 44.1 & - & - & - & - & - & & - & 10.5 & 89.5 & & - & & 0.0 \\
\hline Fisher et al. (2021) & Fisher & $\begin{array}{l}2021-06- \\
09\end{array}$ & UK & 146 & Hospital & NA & 24.0 & - & - & & & & & - & 41.1 & & & - & & 58.9 \\
\hline Ghani et al. (2021) & Ghani & $\begin{array}{l}2021-06- \\
09\end{array}$ & UK & 2,366 & Hospital & NA & & & & & & & & & 33.1 & & & & & 66.9 \\
\hline $\begin{array}{l}\text { Andrade Barreto et } \\
\text { al. (2021) }\end{array}$ & Andrade, Barreto & $\begin{array}{l}2021-06- \\
09\end{array}$ & Brazil & 602 & Community & $\begin{array}{l}51.8^{\wedge} \\
(37-66)\end{array}$ & 59.0 & & & & & & & & 25.6 & & & & & 74.4 \\
\hline $\begin{array}{l}\text { Aabakke et al. } \\
\text { (2021) }\end{array}$ & Aabakke & $\begin{array}{l}2021-06- \\
10\end{array}$ & Denmark & 420 & Community & $\begin{array}{l}29(27- \\
33)\end{array}$ & 100.0 & 2.4 & & - & & & & & & & & & & 51.0 \\
\hline $\begin{array}{l}\text { Yozgat et al. } \\
\text { (2021) }\end{array}$ & Yozgat & $\begin{array}{l}2021-06- \\
10\end{array}$ & Turkey & 382 & Hospital & NA & 37.4 & 29.6 & & & & & & & & & & & & 0.0 \\
\hline $\begin{array}{l}\text { Sandri et al. } \\
\text { (2021) }\end{array}$ & Sandri & $\begin{array}{l}2021-06- \\
10\end{array}$ & Italy & 3,985 & Community & NA & 66.8 & 23.9 & & & & & & & & & & - & & 76.1 \\
\hline Jose et al. (2021) & Jose & $\begin{array}{l}2021-06- \\
10\end{array}$ & USA & 69,264 & $\begin{array}{l}\text { Community } \\
\text { and } \\
\text { Hospital }\end{array}$ & NA & 62.1 & 8.8 & & 1.5 & 0.7 & 25.8 & 0.9 & 1.3 & - & 60.3 & 0.3 & 0.3 & - & 0.0 \\
\hline $\begin{array}{l}\text { Herzberg et al. } \\
\text { (2021) }\end{array}$ & Herzberg & $\begin{array}{l}2021-06- \\
13\end{array}$ & Germany & 52 & Community & $\begin{array}{l}39.7^{\wedge} \\
(27-52)\end{array}$ & 75.0 & - & & - & - & - & - & & 19.2 & - & - & & - & 80.8 \\
\hline Han et al. (2021) & Han, Peng & $\begin{array}{l}2021-06- \\
15\end{array}$ & China & 160 & Hospital & $\begin{array}{l}62.3^{\wedge} \\
(51-73)\end{array}$ & 42.8 & - & & & - & - & - & - & 16.9 & - & - & & - & 83.1 \\
\hline $\begin{array}{l}\text { Abdulamir et al. } \\
\text { (2021) }\end{array}$ & Abdulamir & $\begin{array}{l}2021-06- \\
17\end{array}$ & Iraq & 150 & Hospital & $\begin{array}{l}49.3^{\wedge} \\
(33-65)\end{array}$ & 46.7 & 27.3 & & & - & - & - & - & - & - & - & & - & 0.0 \\
\hline $\begin{array}{l}\text { L. Chauhan et al. } \\
\text { (2021) }\end{array}$ & Chauhan & $\begin{array}{l}2021-06- \\
18\end{array}$ & USA & 376 & Hospital & $\begin{array}{l}58.9^{\wedge} \\
(42-75)\end{array}$ & 43.1 & 6.1 & & - & - & - & - & - & - & - & - & & - & 93.9 \\
\hline $\begin{array}{l}\text { Barchuk et al. } \\
\text { (2021) }\end{array}$ & Barchuk & $\begin{array}{l}2021-06- \\
21\end{array}$ & Russia & 962 & Community & NA & 62.8 & 20.3 & & - & - & 27.8 & - & - & - & 48.5 & - & & - & 3.4 \\
\hline $\begin{array}{l}\text { Patone et al. } \\
\text { (2021) }\end{array}$ & Patone & $\begin{array}{l}2021-06- \\
22\end{array}$ & UK & 429,926 & $\begin{array}{l}\text { Community } \\
\text { and } \\
\text { Hospital }\end{array}$ & $\begin{array}{l}37.7^{\wedge} \\
(19-55)\end{array}$ & 52.7 & 11.2 & & & & 17.6 & & - & & 56.3 & & - & & 15.0 \\
\hline $\begin{array}{l}\text { Mollan et al. } \\
\text { (2021) }\end{array}$ & Mollan & $\begin{array}{l}2021-06- \\
25\end{array}$ & USA & 204 & Community & $\begin{array}{l}40(27- \\
52)\end{array}$ & 51.0 & & & 14.7 & & & 16.7 & & & & 68.1 & - & & 0.5 \\
\hline $\begin{array}{l}\text { Sonnweber et al. } \\
\text { (2021) }\end{array}$ & Sonnweber & $\begin{array}{l}2021-06- \\
25\end{array}$ & Austria & 108 & Hospital & $\begin{array}{l}57^{\wedge} \\
(43-71)\end{array}$ & 45.4 & 3.7 & - & & & 42.6 & & & & & & - & & 53.7 \\
\hline $\begin{array}{l}\text { Nassar et al. } \\
\text { (2021) }\end{array}$ & Nassar & $\begin{array}{l}2021-06- \\
25\end{array}$ & Egypt & 160 & Hospital & $\begin{array}{l}60^{\wedge} \\
(46-74)\end{array}$ & 32.5 & 10.6 & & - & - & - & & - & - & - & - & & - & 89.4 \\
\hline Ali et al. (2021) & Ali, Hasan & $\begin{array}{l}2021-06- \\
25\end{array}$ & Bangladesh & 326 & Hospital & NA & 39.9 & 20.9 & & - & - & - & - & - & - & - & - & & 79.1 & 0.0 \\
\hline $\begin{array}{l}\text { Demichev et al. } \\
\text { (2021) }\end{array}$ & Demichev & $\begin{array}{l}2021-06- \\
26\end{array}$ & Multiple & 50 & Hospital & $\begin{array}{l}62(54- \\
73)\end{array}$ & 30.0 & 2.0 & & - & - & 16.0 & - & - & - & 82.0 & - & & - & 0.0 \\
\hline $\begin{array}{l}\text { Mostafa et al. } \\
\text { (2021) }\end{array}$ & Mostafa & $\begin{array}{l}2021-06- \\
28\end{array}$ & Egypt & 4,040 & Community & NA & 61.5 & 11.9 & & - & - & 2.0 & - & - & - & 86.2 & - & & - & 0.0 \\
\hline $\begin{array}{l}\text { Tehrani et al. } \\
\text { (2021a) }\end{array}$ & Tehrani & $\begin{array}{l}2021-06- \\
30\end{array}$ & USA & 8,222 & Hospital & NA & 44.0 & - & & & - & - & - & - & 6.3 & - & - & & - & 93.7 \\
\hline $\begin{array}{l}\text { Tehrani et al. } \\
\text { (2021b) }\end{array}$ & Tehrani & $\begin{array}{l}2021-06- \\
30\end{array}$ & USA & 8,222 & Hospital & NA & 44.0 & - & - & - & - & & & - & 6.3 & & & - & & 93.7 \\
\hline Amer et al. (2021) & Amer & $\begin{array}{l}2021-07- \\
n+1-\end{array}$ & Multiple & 860 & Hospital & NA & 34.0 & 4.9 & 0.1 & - & - & 20.1 & & - & & & & - & & 74.9 \\
\hline
\end{tabular}




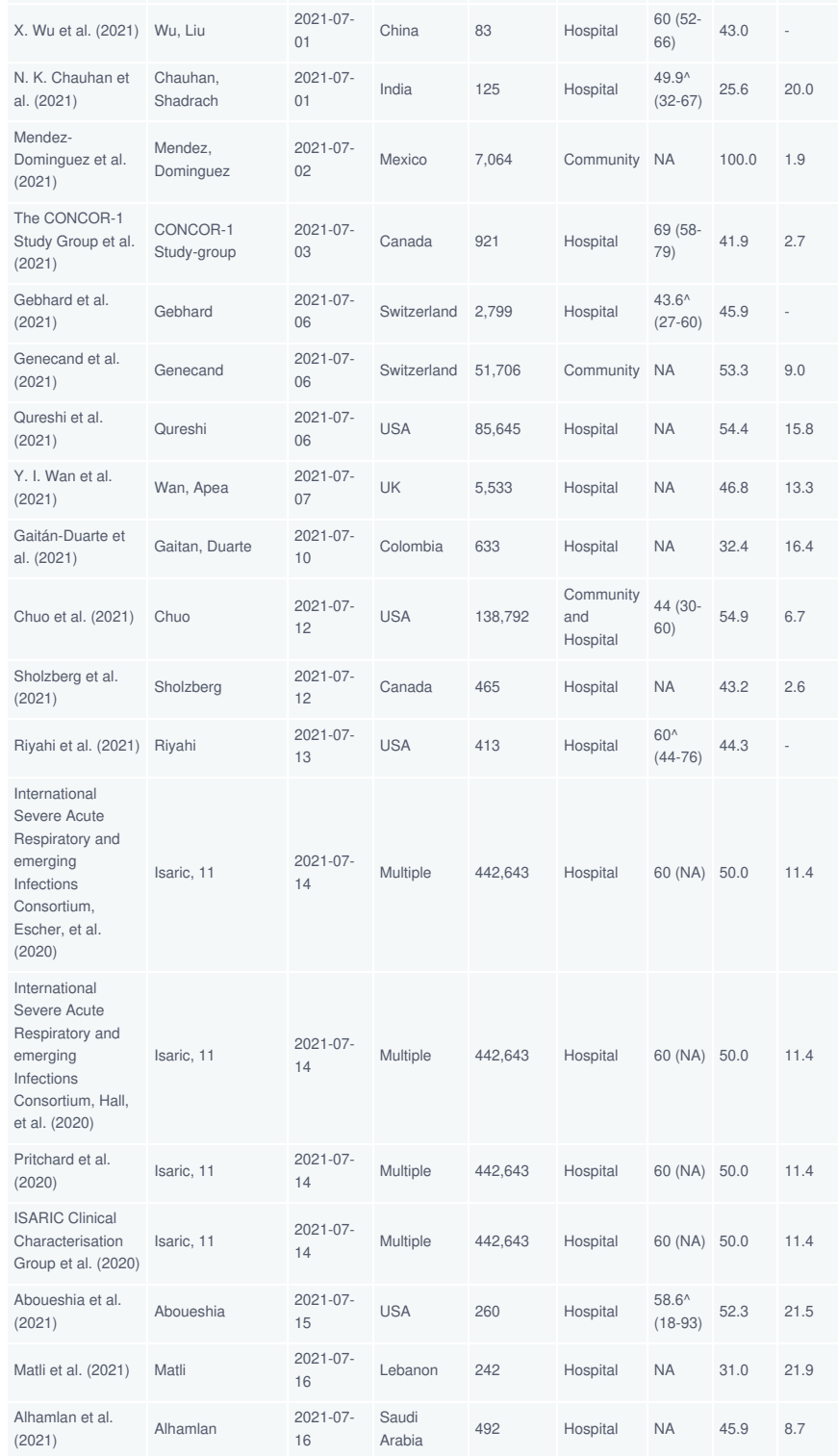

Smoking prevalence by country

Unadjusted smoking prevalence compared with overall estimates for national adult smoking prevalence split by country and study setting is presented in Figure 2. Lower than expected current smoking prevalence was generally observed, especially in studies with hospitalised samples. Former smoking prevalence was more similar to expected prevalence when reported; however, studybased prevalence was typically higher than national estimates. National smoking prevalence estimates used for comparison are presented in Supplementary table 3.

Hospital - current

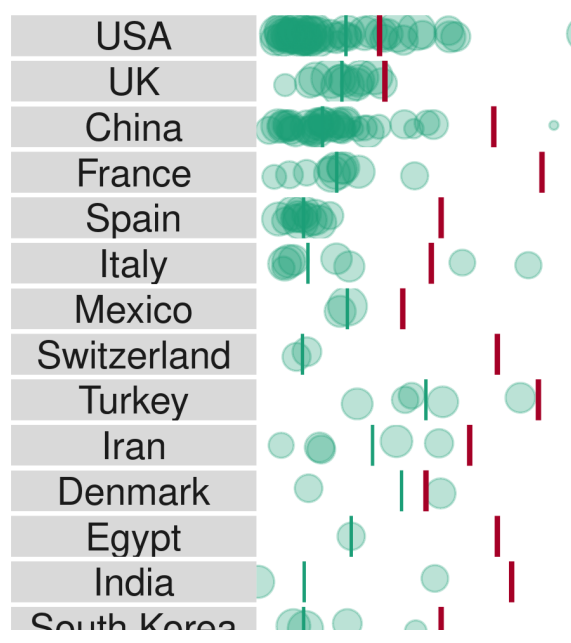

Hospital - former

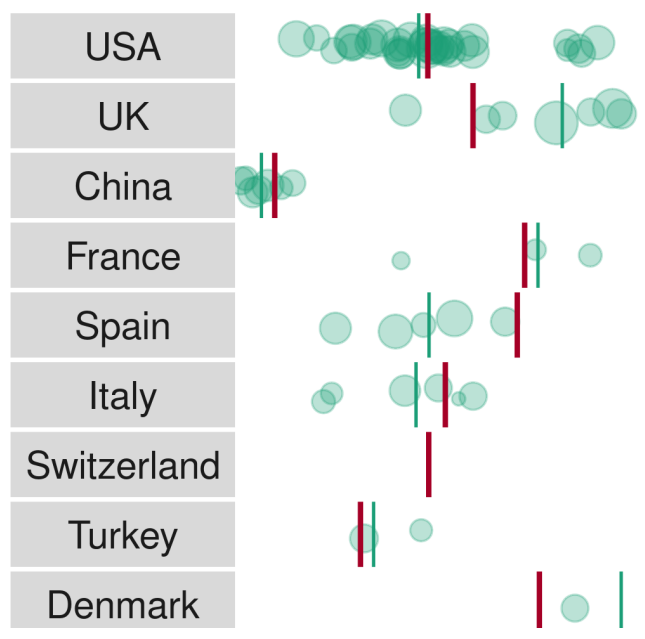




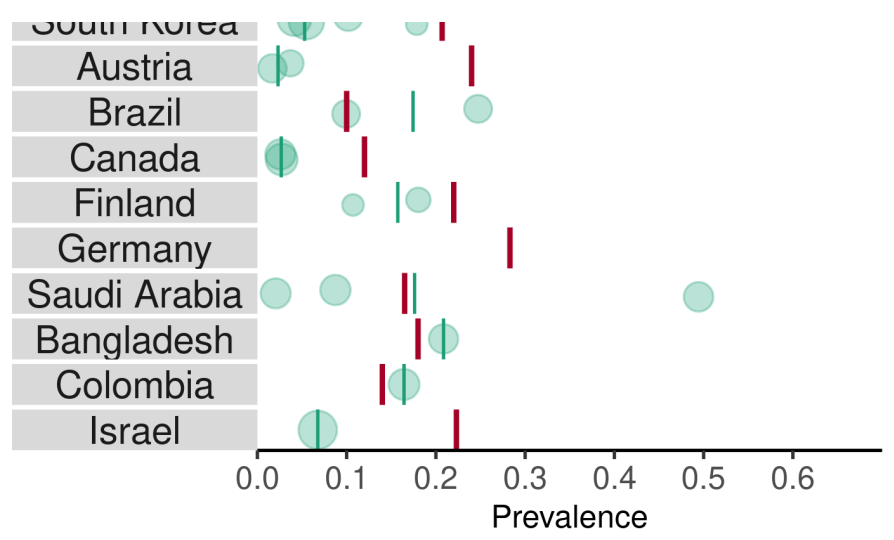

Community and hospital - current

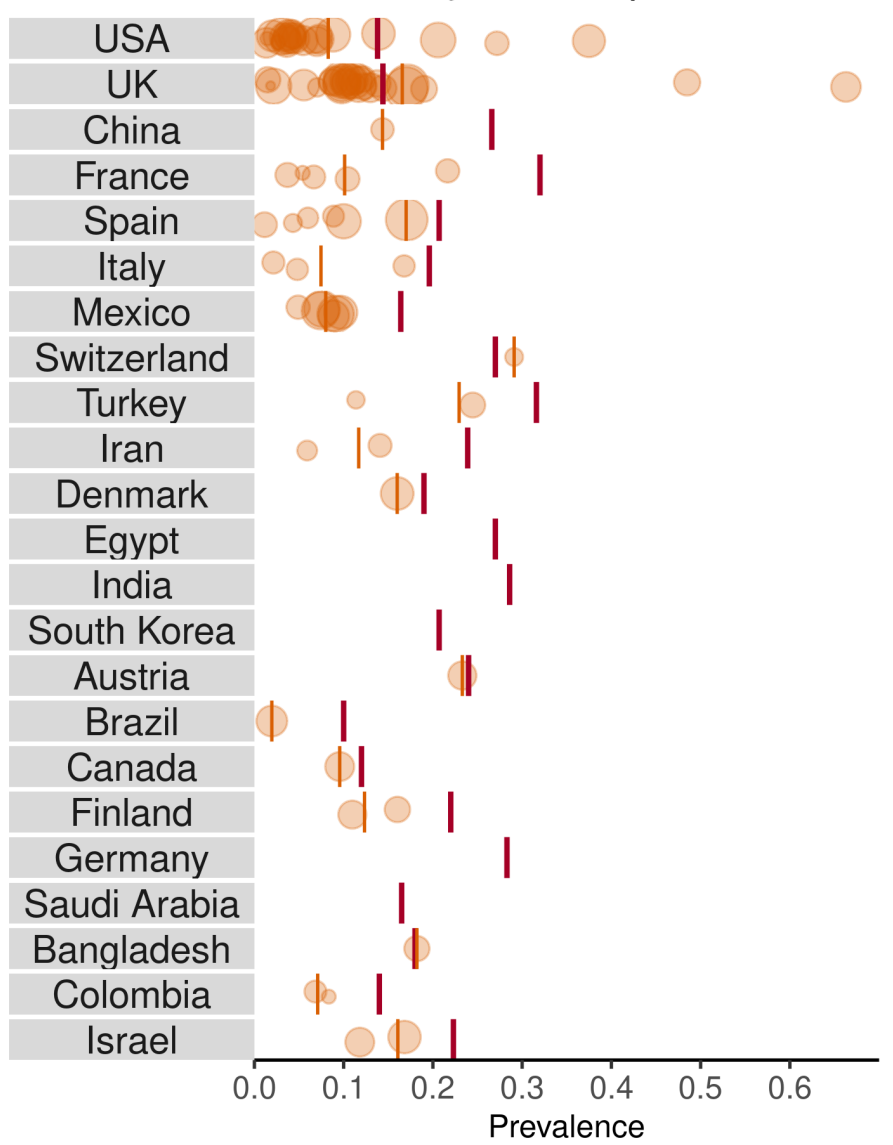

Community - current

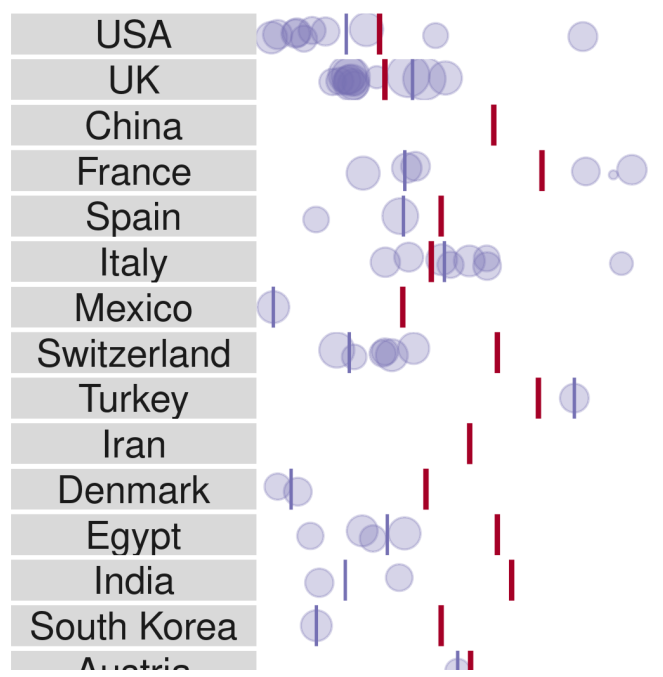

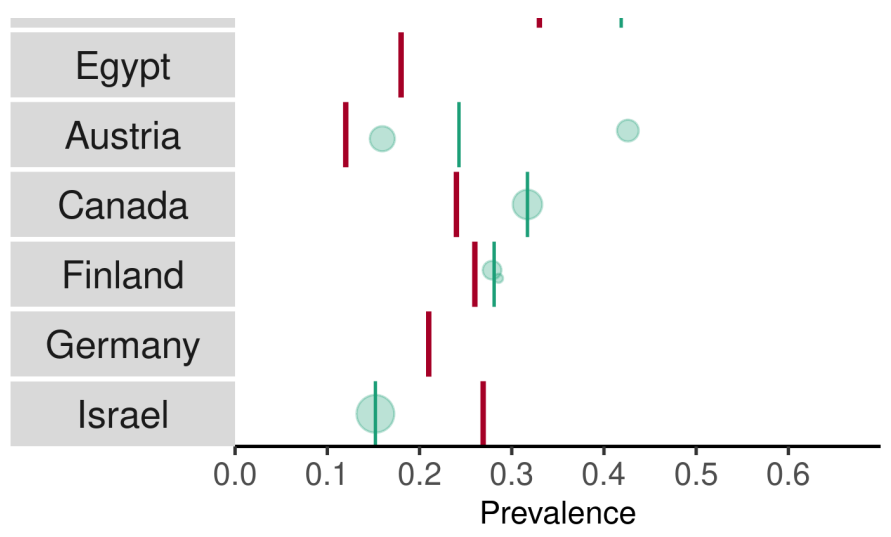

Community and hospital - former

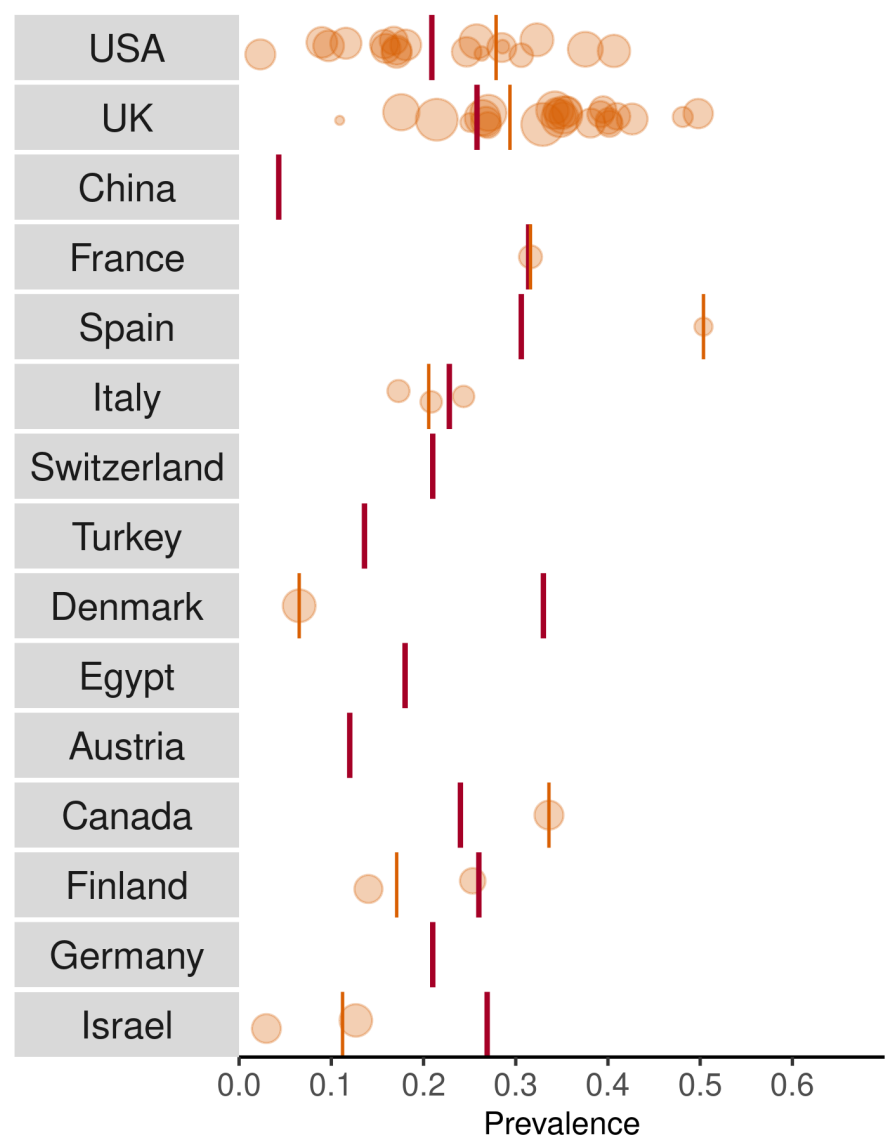

Community - former

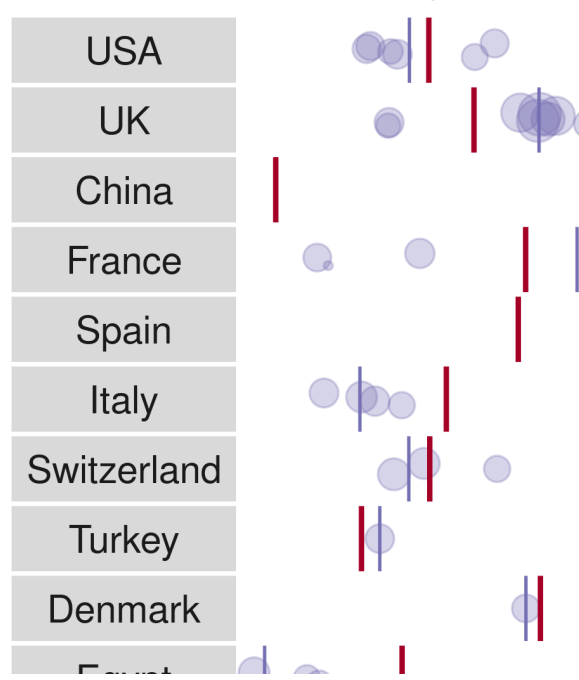



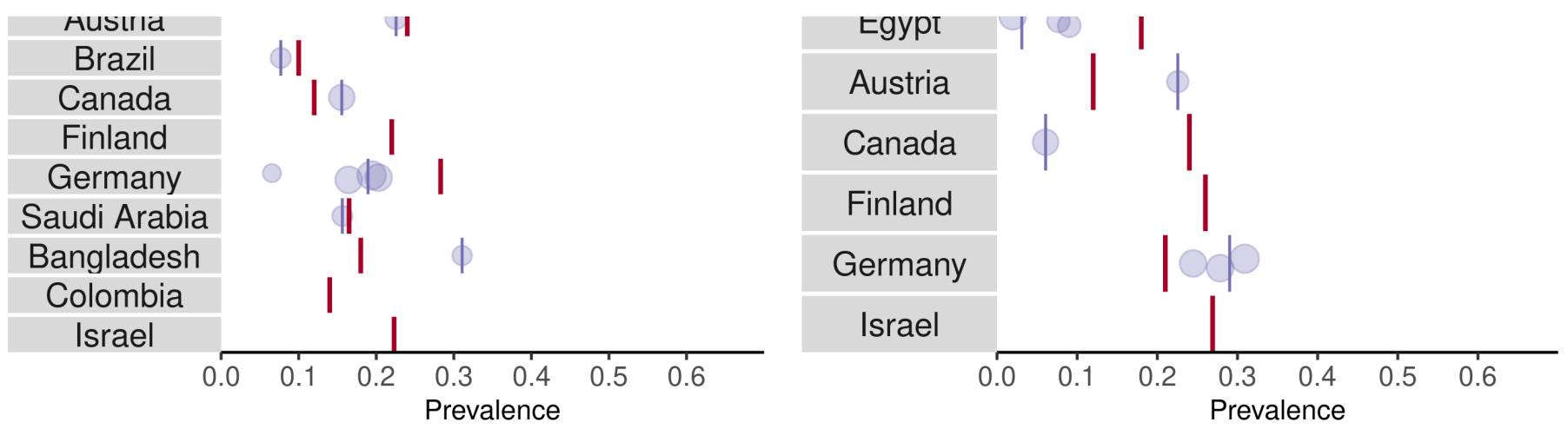

Figure 2. Weighted mean prevalence of current (left panel) and former (right panel) smoking in included studies, split by country. The circles represent individual studies, with colour corresponding to study setting (i.e. hospital, community and hospital, community) and size corresponding to relative study sample size. For comparison, national current and former smoking prevalence are indicated by the red lines. Countries with three or more eligible studies are shown.

SARS-CoV-2 testing by smoking status

Four studies provided data on access to SARS-CoV-2 diagnostic testing for those meeting local testing criteria by smoking status. In a cohort study of US military veterans aged 54-75 (Rentsch et al., 2020b), current smokers were more likely to receive a test: $42.3 \%(1,603 / 3,789)$ of the sample were current smokers compared with $23.8 \%$ of all veterans aged $50+$ years using any tobacco product between 2010-2015 (Odani, 2018). In the UK Biobank cohort (Niedzwiedz et al., 2020), a multivariable analysis showed former $(\mathrm{RR}=1.29,95 \% \mathrm{Cl}=1.14-1.45, p<.001)$ and current $(\mathrm{RR}=$ $1.44,95 \% \mathrm{Cl}=1.20-1.71, p<.001$ ) compared with never smokers to be more likely to receive a test. In an Australian rapid assessment screening clinic for COVID-19 (Trubiano et al., 2020 ), $9.4 \%$ $(397 / 4,226)$ of the self-referred sample (subsequently assessed by a healthcare professional to decide on testing) were current smokers. Of these self-referrals, healthcare professionals decided that current compared with former or never smokers were less likely to require a test ( $\mathrm{RR}=0.93,95 \% \mathrm{Cl}=0.86-1.0, p=0.045)$. In a further study using the UK Biobank cohort (Didikoglu et al., 2021), current $(\mathrm{RR}=1.23,95 \% \mathrm{Cl}=1.19-1.26, p<0.001)$ and former smokers $(\mathrm{RR}=1.20,95 \% \mathrm{Cl}=1.18-1.23 p<0.001)$ were more likely to receive a test compared with never smokers.

\section{SARS-CoV-2 infection by smoking status}

108 studies provided data on SARS-CoV-2 infection for people meeting local testing criteria by smoking status (see Table 2). Meta-analyses were performed for 6 'good' and 33 'fair' quality studies (see Figure 3 and 4). Current smokers were at reduced risk of testing positive for SARS-CoV-2 compared with never smokers $(\mathrm{RR}=0.67,95 \% \mathrm{Credible}$ Interval $(\mathrm{Crl})=0.6-0.75, \mathrm{~T}=0.27)$. The six good quality studies each reported point estimates less than 1 , although the $\mathrm{Crl}$ for two of the six studies crossed 1 for current smokers (see starred studies in Figure 3 ). The probability of current smokers being at reduced risk of infection compared with never smokers ( $R R \leq 0.9)$ was $>99 \%$. Former compared with never smokers were had an equivalent risk of testing positive, with data being inconclusive ( $R R=0.99,95 \% \mathrm{Crl}=0.94-1.05, \mathrm{~T}=0.12)$ ), favouring there being no important association. The probability of former smokers being at increased risk of infection ( $R R \geq 1.1)$ compared with never smokers was $<1 \%$. Results were materially unchanged in sensitivity analyses.

Table 2 SARS-CoV-2 infection by smoking status

\begin{tabular}{|c|c|c|c|c|c|c|c|c|c|c|c|c|c|c|c|c|c|}
\hline \multirow[b]{2}{*}{ Author } & \multirow[b]{2}{*}{$\begin{array}{l}\text { Total } \\
\text { population } \\
\text { tested }\end{array}$} & \multicolumn{8}{|c|}{ SARS-CoV-2 negative } & \multicolumn{8}{|c|}{ SARS-CoV-2 positive } \\
\hline & & $\mathrm{N}(\%)$ & $\begin{array}{l}\text { Current } \\
\text { smoker } \\
(\%)\end{array}$ & $\begin{array}{l}\text { Former } \\
\text { smoker } \\
(\%)\end{array}$ & $\begin{array}{l}\text { Current/former } \\
\text { smoker }(\%)\end{array}$ & $\begin{array}{l}\text { Never } \\
\text { smoker } \\
\text { (\%) }\end{array}$ & $\begin{array}{l}\text { Current } \\
\text { vaper } \\
(\%)\end{array}$ & $\begin{array}{l}\text { Current vaper } \\
\text { and smoker (\%) }\end{array}$ & $\begin{array}{l}\text { Not } \\
\text { stated } \\
(\%)\end{array}$ & $\mathrm{N}(\%)$ & $\begin{array}{l}\text { Current } \\
\text { smoker } \\
(\%)\end{array}$ & $\begin{array}{l}\text { Former } \\
\text { smoker } \\
(\%)\end{array}$ & $\begin{array}{l}\text { Current/former } \\
\text { smoker }(\%)\end{array}$ & $\begin{array}{l}\text { Never } \\
\text { smoker } \\
(\%)\end{array}$ & $\begin{array}{l}\text { Current } \\
\text { vaper } \\
(\%)\end{array}$ & $\begin{array}{l}\text { Current vaper } \\
\text { and smoker (\%) }\end{array}$ & $\begin{array}{l}\text { Not } \\
\text { stated } \\
(\%)\end{array}$ \\
\hline Rentsch & 3,528 & $\begin{array}{l}2974 \\
(84.30 \%)\end{array}$ & $\begin{array}{l}1444 \\
(48.55 \%)\end{array}$ & $\begin{array}{l}704 \\
(23.67 \%)\end{array}$ & & $\begin{array}{l}826 \\
(27.77 \%)\end{array}$ & & & . & $\begin{array}{l}554 \\
(15.70 \%)\end{array}$ & $\begin{array}{l}159 \\
(28.70 \%)\end{array}$ & $\begin{array}{l}179 \\
(32.31 \%)\end{array}$ & & $\begin{array}{l}216 \\
(38.99 \%)\end{array}$ & & & \\
\hline Fontanet & 661 & $\begin{array}{l}490 \\
(74.13 \%)\end{array}$ & $\begin{array}{l}64 \\
(13.06 \%)\end{array}$ & - & & $\begin{array}{l}426 \\
(86.94 \%)\end{array}$ & & & - & $\begin{array}{l}171 \\
(25.87 \%)\end{array}$ & $5(2.92 \%)$ & & - & $\begin{array}{l}166 \\
(97.08 \%)\end{array}$ & - & - & - \\
\hline Cho & 1,331 & $\begin{array}{l}793 \\
(59.58 \%)\end{array}$ & $\begin{array}{l}142 \\
(17.91 \%)\end{array}$ & $\begin{array}{l}214 \\
(26.99 \%)\end{array}$ & - & $\begin{array}{l}437 \\
(55.11 \%)\end{array}$ & - & - & & $\begin{array}{l}538 \\
(40.42 \%)\end{array}$ & $\begin{array}{l}111 \\
(20.63 \%)\end{array}$ & $\begin{array}{l}145 \\
(26.95 \%)\end{array}$ & - & $\begin{array}{l}282 \\
(52.42 \%)\end{array}$ & - & - & \\
\hline Shah & 243 & $\begin{array}{l}212 \\
(87.24 \%)\end{array}$ & $\begin{array}{l}52 \\
(24.53 \%)\end{array}$ & $\begin{array}{l}47 \\
(22.17 \%)\end{array}$ & & $\begin{array}{l}113 \\
(53.30 \%)\end{array}$ & & & - & $\begin{array}{l}29 \\
(11.93 \%)\end{array}$ & $0(0.00 \%)$ & $\begin{array}{l}9 \\
(31.03 \%)\end{array}$ & & $\begin{array}{l}20 \\
(68.97 \%)\end{array}$ & & & \\
\hline Kolin & 1,474 & $\begin{array}{l}805 \\
(54.61 \%)\end{array}$ & $\begin{array}{l}141 \\
(17.52 \%)\end{array}$ & $\begin{array}{l}307 \\
(38.14 \%)\end{array}$ & & $\begin{array}{l}354 \\
(43.98 \%)\end{array}$ & & & $3(0.37 \%)$ & $\begin{array}{l}669 \\
(45.39 \%)\end{array}$ & $\begin{array}{l}72 \\
(10.76 \%)\end{array}$ & $\begin{array}{l}285 \\
(42.60 \%)\end{array}$ & - & $\begin{array}{l}303 \\
(45.29 \%)\end{array}$ & - & - & $\begin{array}{l}9 \\
(1.35 \%)\end{array}$ \\
\hline de Lusignan & 3,291 & $\begin{array}{l}2740 \\
(83.26 \%)\end{array}$ & $\begin{array}{l}366 \\
(13.36 \%)\end{array}$ & $\begin{array}{l}1450 \\
(52.92 \%)\end{array}$ & & $\begin{array}{l}924 \\
(33.72 \%)\end{array}$ & & & - & $\begin{array}{l}551 \\
(16.74 \%)\end{array}$ & $47(8.53 \%)$ & $\begin{array}{l}303 \\
(54.99 \%)\end{array}$ & - & $\begin{array}{l}201 \\
(36.48 \%)\end{array}$ & - & - & - \\
\hline Valenti & 789 & $\begin{array}{l}689 \\
(87.33 \%)\end{array}$ & $\begin{array}{l}197 \\
(28.59 \%)\end{array}$ & - & - & & - & - & $\begin{array}{l}492 \\
(71.41 \%)\end{array}$ & $\begin{array}{l}40 \\
(5.07 \%)\end{array}$ & $7(17.50 \%)$ & - & - & - & - & - & $\begin{array}{l}33 \\
(82.50 \%)\end{array}$ \\
\hline Parrotta & 76 & $\begin{array}{l}39 \\
(51.32 \%)\end{array}$ & $1(2.56 \%)$ & $\begin{array}{l}10 \\
(25.64 \%)\end{array}$ & & $\begin{array}{l}27 \\
(69.23 \%)\end{array}$ & & & $1(2.56 \%)$ & $\begin{array}{l}37 \\
(48.68 \%)\end{array}$ & $1(2.70 \%)$ & $\begin{array}{l}10 \\
(27.03 \%)\end{array}$ & & $\begin{array}{l}25 \\
(67.57 \%)\end{array}$ & & & $\begin{array}{l}1 \\
(2.70 \%)\end{array}$ \\
\hline Berumen & 102,875 & $\begin{array}{l}71353 \\
(69.36 \%)\end{array}$ & & - & $7173(10.05 \%)$ & $\begin{array}{l}64180 \\
(89.95 \%)\end{array}$ & & & - & $\begin{array}{l}31522 \\
(30.64 \%)\end{array}$ & - & & $2748(8.72 \%)$ & $\begin{array}{l}28774 \\
(91.28 \%)\end{array}$ & - & - & - \\
\hline Israel & 24,906 & $\begin{array}{l}20755 \\
(83.33 \%)\end{array}$ & $\begin{array}{l}3783 \\
(18.23 \%)\end{array}$ & $\begin{array}{l}2671 \\
(12.87 \%)\end{array}$ & - & $\begin{array}{l}14301 \\
(68.90 \%)\end{array}$ & - & - & & $\begin{array}{l}4151 \\
(16.67 \%)\end{array}$ & $\begin{array}{l}406 \\
(9.78 \%)\end{array}$ & $\begin{array}{l}483 \\
(11.64 \%)\end{array}$ & - & $\begin{array}{l}3262 \\
(78.58 \%)\end{array}$ & - & - & - \\
\hline del Valle & 1,108 & $\begin{array}{l}143 \\
(12.91 \%)\end{array}$ & $\begin{array}{l}27 \\
(18.88 \%)\end{array}$ & $\begin{array}{l}53 \\
(37.06 \%)\end{array}$ & - & & - & - & $\begin{array}{l}63 \\
(44.06 \%)\end{array}$ & $\begin{array}{l}965 \\
(87.09 \%)\end{array}$ & $55(5.70 \%)$ & $\begin{array}{l}293 \\
(30.36 \%)\end{array}$ & - & - & - & - & $\begin{array}{l}617 \\
(63.94 \%)\end{array}$ \\
\hline Romao & 34 & $\begin{array}{l}20 \\
(58.82 \%)\end{array}$ & & & $5(25.00 \%)$ & - & & & $\begin{array}{l}15 \\
(75.00 \%)\end{array}$ & $\begin{array}{l}14 \\
(41.18 \%)\end{array}$ & & & $4(28.57 \%)$ & & & & $\begin{array}{l}10 \\
(71.43 \%)\end{array}$ \\
\hline Ramlall & 11,116 & $\begin{array}{l}4723 \\
(42.49 \%)\end{array}$ & . & - & & $\cdot$ & & & - & $\begin{array}{l}6393 \\
(57.51 \%)\end{array}$ & - & & $\begin{array}{l}1643.001 \\
(25.70 \%)\end{array}$ & $\begin{array}{l}4749.999 \\
(74.30 \%)\end{array}$ & - & - & - \\
\hline Sharma & 501 & $\begin{array}{l}267 \\
(53.29 \%)\end{array}$ & - & - & $1(0.37 \%)$ & & - & - & $\begin{array}{l}266 \\
(99.63 \%)\end{array}$ & $\begin{array}{l}234 \\
(46.71 \%)\end{array}$ & - & - & $20(8.55 \%)$ & - & - & - & $\begin{array}{l}214 \\
(91.45 \%)\end{array}$ \\
\hline Eugen-Olsen & 407 & $\begin{array}{l}290 \\
(71.25 \%)\end{array}$ & $\begin{array}{l}76 \\
(26.21 \%)\end{array}$ & $\begin{array}{l}104 \\
(35.86 \%)\end{array}$ & & $\begin{array}{l}102 \\
(35.17 \%)\end{array}$ & & & - & $\begin{array}{l}117 \\
(28.75 \%)\end{array}$ & $8(6.84 \%)$ & $\begin{array}{l}46 \\
(39.32 \%)\end{array}$ & & $\begin{array}{l}59 \\
(50.43 \%)\end{array}$ & - & & \\
\hline & & 3184 & & & & & & & 1531 & 1326 & & & & & & & 64.3 \\
\hline
\end{tabular}




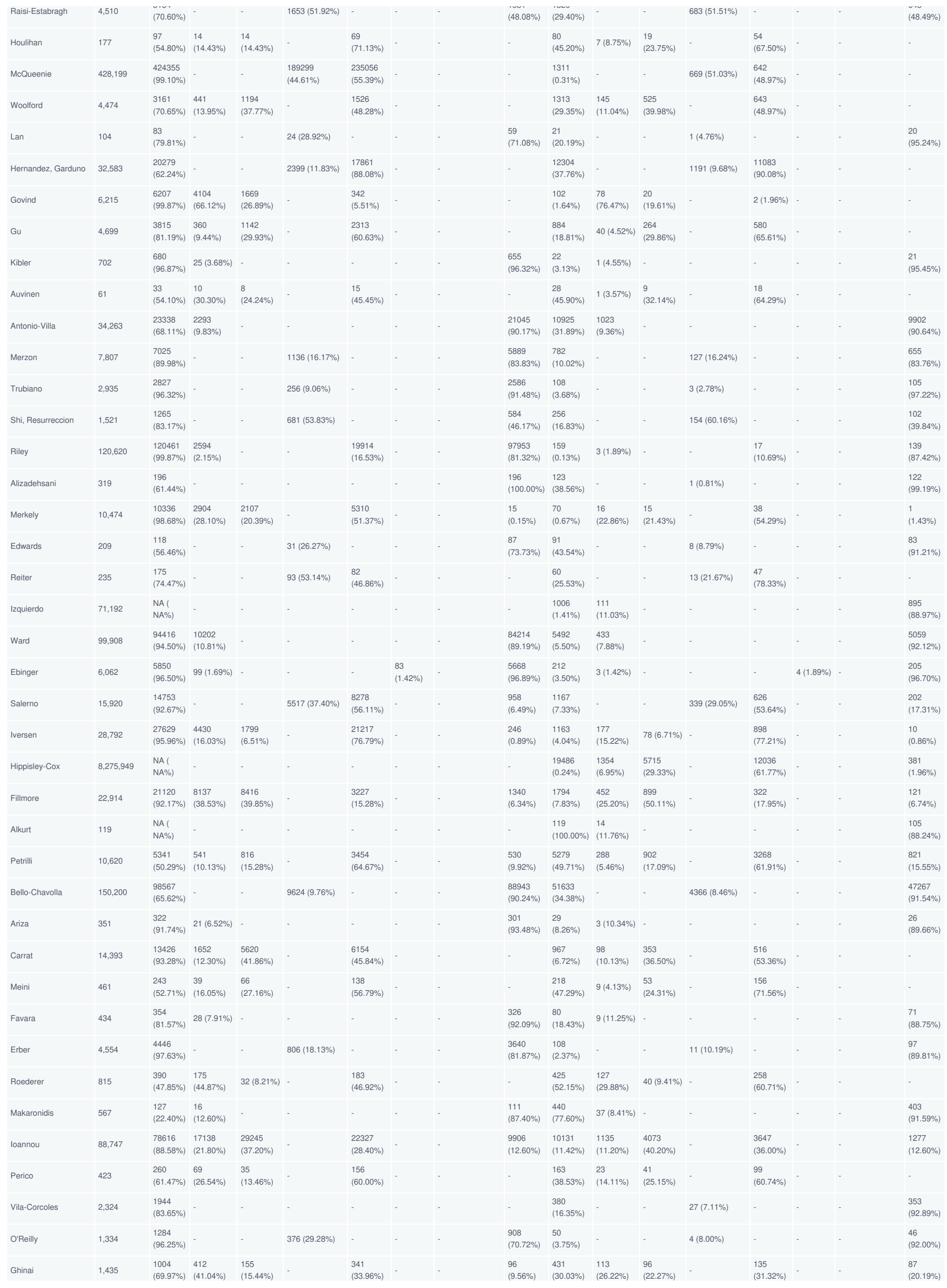




\begin{tabular}{|c|c|c|c|c|c|c|c|c|c|c|c|c|c|c|c|c|c|}
\hline Kortela & 2,993 & $\begin{array}{l}2419 \\
(80.82 \%)\end{array}$ & $\begin{array}{l}300 \\
(12.40 \%)\end{array}$ & $\begin{array}{l}340 \\
(14.06 \%)\end{array}$ & & $\begin{array}{l}636 \\
(26.29 \%)\end{array}$ & - & & $\begin{array}{l}1143 \\
(47.25 \%)\end{array}$ & $\begin{array}{l}574 \\
(19.18 \%)\end{array}$ & $26(4.53 \%)$ & $\begin{array}{l}80 \\
(13.94 \%)\end{array}$ & - & $\begin{array}{l}232 \\
(40.42 \%)\end{array}$ & & & $\begin{array}{l}236 \\
(41.11 \%)\end{array}$ \\
\hline Gallichotte & 239 & $\begin{array}{l}190 \\
(79.50 \%)\end{array}$ & $\begin{array}{l}40 \\
(21.05 \%)\end{array}$ & $\begin{array}{l}28 \\
(14.74 \%)\end{array}$ & & $\begin{array}{l}110 \\
(57.89 \%)\end{array}$ & $\begin{array}{l}12 \\
(6.32 \%)\end{array}$ & & & $\begin{array}{l}49 \\
(20.50 \%)\end{array}$ & $8(16.33 \%)$ & $\begin{array}{l}12 \\
(24.49 \%)\end{array}$ & - & $\begin{array}{l}27 \\
(55.10 \%)\end{array}$ & $2(4.08 \%)$ & & \\
\hline Saeed & 173 & $\begin{array}{l}69 \\
(39.88 \%)\end{array}$ & $2(2.90 \%)$ & & & & & & $\begin{array}{l}67 \\
(97.10 \%)\end{array}$ & $\begin{array}{l}104 \\
(60.12 \%)\end{array}$ & $9(8.65 \%)$ & - & & & & & $\begin{array}{l}95 \\
(91.35 \%)\end{array}$ \\
\hline Woolcott & $1,636,050$ & $\begin{array}{l}878840 \\
(53.72 \%)\end{array}$ & . & - & $85816(9.76 \%)$ & - & - & - & $\begin{array}{l}793024 \\
(90.24 \%)\end{array}$ & $\begin{array}{l}757210 \\
(46.28 \%)\end{array}$ & - & & $57451(7.59 \%)$ & - & - & - & $\begin{array}{l}699759 \\
(92.41 \%)\end{array}$ \\
\hline Barasa & 394 & $\begin{array}{l}277 \\
(70.30 \%)\end{array}$ & $\begin{array}{l}49 \\
(17.69 \%)\end{array}$ & $\begin{array}{l}105 \\
(37.91 \%)\end{array}$ & - & $\begin{array}{l}110 \\
(39.71 \%)\end{array}$ & - & - & $\begin{array}{l}13 \\
(4.69 \%)\end{array}$ & $\begin{array}{l}117 \\
(29.70 \%)\end{array}$ & $9(7.69 \%)$ & $\begin{array}{l}37 \\
(31.62 \%)\end{array}$ & & $\begin{array}{l}54 \\
(46.15 \%)\end{array}$ & - & - & $\begin{array}{l}17 \\
(14.53 \%)\end{array}$ \\
\hline Paleiron & 1,688 & $\begin{array}{l}409 \\
(24.23 \%)\end{array}$ & $\begin{array}{l}236 \\
(57.70 \%)\end{array}$ & $\begin{array}{l}77 \\
(18.83 \%)\end{array}$ & & $\begin{array}{l}96 \\
(23.47 \%)\end{array}$ & - & & & $\begin{array}{l}1279 \\
(75.77 \%)\end{array}$ & $\begin{array}{l}579 \\
(45.27 \%)\end{array}$ & $\begin{array}{l}309 \\
(24.16 \%)\end{array}$ & - & $\begin{array}{l}391 \\
(30.57 \%)\end{array}$ & & & \\
\hline Didikoglu & 43,428 & $\begin{array}{l}35695 \\
(82.19 \%)\end{array}$ & $\begin{array}{l}3919 \\
(10.98 \%)\end{array}$ & $\begin{array}{l}13841 \\
(38.78 \%)\end{array}$ & & $\begin{array}{l}17939 \\
(50.26 \%)\end{array}$ & & & & $\begin{array}{l}7733 \\
(17.81 \%)\end{array}$ & $\begin{array}{l}867 \\
(11.21 \%)\end{array}$ & $\begin{array}{l}2966 \\
(38.36 \%)\end{array}$ & & $\begin{array}{l}3901 \\
(50.45 \%)\end{array}$ & & & \\
\hline Kantele & 1,095 & $\begin{array}{l}1059 \\
(96.71 \%)\end{array}$ & $\begin{array}{l}176 \\
(16.62 \%)\end{array}$ & $\begin{array}{l}272 \\
(25.68 \%)\end{array}$ & - & $\begin{array}{l}611 \\
(57.70 \%)\end{array}$ & - & - & - & $\begin{array}{l}36 \\
(3.29 \%)\end{array}$ & $3(8.33 \%)$ & $\begin{array}{l}11 \\
(30.56 \%)\end{array}$ & - & $\begin{array}{l}21 \\
(58.33 \%)\end{array}$ & - & - & $\begin{array}{l}1 \\
(2.78 \%)\end{array}$ \\
\hline Polat & 1,322 & $\begin{array}{l}\text { NA ( } \\
\text { NA\%) }\end{array}$ & - & - & - & - & & - & - & $\begin{array}{l}23 \\
(1.74 \%)\end{array}$ & $4(17.39 \%)$ & $2(8.70 \%)$ & & $\begin{array}{l}17 \\
(73.91 \%)\end{array}$ & - & - & - \\
\hline Richard & 8,344 & $\begin{array}{l}6798 \\
(81.47 \%)\end{array}$ & $\begin{array}{l}1065 \\
(15.67 \%)\end{array}$ & $\begin{array}{l}1171 \\
(17.23 \%)\end{array}$ & & $\begin{array}{l}4456 \\
(65.55 \%)\end{array}$ & - & & $\begin{array}{l}106 \\
(1.56 \%)\end{array}$ & $\begin{array}{l}531 \\
(6.36 \%)\end{array}$ & $47(8.85 \%)$ & $\begin{array}{l}83 \\
(15.63 \%)\end{array}$ & - & $\begin{array}{l}396 \\
(74.58 \%)\end{array}$ & & & $\begin{array}{l}5 \\
(0.94 \%)\end{array}$ \\
\hline Schubl & 1,557 & $\begin{array}{l}1392 \\
(89.40 \%)\end{array}$ & $33(2.37 \%)$ & & & & & & $\begin{array}{l}1359 \\
(97.63 \%)\end{array}$ & $\begin{array}{l}165 \\
(10.60 \%)\end{array}$ & $4(2.42 \%)$ & - & & & & & $\begin{array}{l}161 \\
(97.58 \%)\end{array}$ \\
\hline Nezhadmoghadam & 33,325 & $\begin{array}{l}19958 \\
(59.89 \%)\end{array}$ & $\begin{array}{l}2151 \\
(10.78 \%)\end{array}$ & - & - & & - & & $\begin{array}{l}17807 \\
(89.22 \%)\end{array}$ & $\begin{array}{l}13367 \\
(40.11 \%)\end{array}$ & $\begin{array}{l}1251 \\
(9.36 \%)\end{array}$ & - & - & & & & $\begin{array}{l}12116 \\
(90.64 \%)\end{array}$ \\
\hline Mora & 1,058 & $\begin{array}{l}857 \\
(81.00 \%)\end{array}$ & $40(4.67 \%)$ & $\begin{array}{l}118 \\
(13.77 \%)\end{array}$ & - & $\begin{array}{l}698 \\
(81.45 \%)\end{array}$ & & - & $1(0.12 \%)$ & $\begin{array}{l}201 \\
(19.00 \%)\end{array}$ & $8(3.98 \%)$ & $\begin{array}{l}36 \\
(17.91 \%)\end{array}$ & & $\begin{array}{l}157 \\
(78.11 \%)\end{array}$ & - & - & - \\
\hline Molenaar & 696 & $\begin{array}{l}591 \\
(84.91 \%)\end{array}$ & $29(4.91 \%)$ & - & - & - & - & - & $\begin{array}{l}562 \\
(95.09 \%)\end{array}$ & $\begin{array}{l}105 \\
(15.09 \%)\end{array}$ & $2(1.90 \%)$ & - & - & - & - & - & $\begin{array}{l}103 \\
(98.10 \%)\end{array}$ \\
\hline $\begin{array}{l}\text { Vila-Corcoles, Satue- } \\
\text { Gracia, Vila-Rovira }\end{array}$ & 4,113 & $\begin{array}{l}3577 \\
(86.97 \%)\end{array}$ & $\begin{array}{l}591 \\
(16.52 \%)\end{array}$ & - & & - & - & & $\begin{array}{l}2986 \\
(83.48 \%)\end{array}$ & $\begin{array}{l}536 \\
(13.03 \%)\end{array}$ & $41(7.65 \%)$ & - & - & & - & & $\begin{array}{l}495 \\
(92.35 \%)\end{array}$ \\
\hline Gegout Petit & 2,006 & $\begin{array}{l}1964 \\
(97.91 \%)\end{array}$ & $\begin{array}{l}334 \\
(17.01 \%)\end{array}$ & & & $\begin{array}{l}1545 \\
(78.67 \%)\end{array}$ & & & $\begin{array}{l}85 \\
(4.33 \%)\end{array}$ & $\begin{array}{l}42 \\
(2.09 \%)\end{array}$ & $4(9.52 \%)$ & & & $\begin{array}{l}38 \\
(90.48 \%)\end{array}$ & & & \\
\hline Leister & 3,301 & $\begin{array}{l}3269 \\
(99.03 \%)\end{array}$ & $\begin{array}{l}764 \\
(23.37 \%)\end{array}$ & - & - & - & - & - & $\begin{array}{l}2505 \\
(76.63 \%)\end{array}$ & $\begin{array}{l}32 \\
(0.97 \%)\end{array}$ & $5(15.62 \%)$ & & & - & - & - & $\begin{array}{l}27 \\
(84.38 \%)\end{array}$ \\
\hline Bell & 303 & $\begin{array}{l}\text { NA ( } \\
\text { NA\%) }\end{array}$ & - & - & - & - & & - & - & $\begin{array}{l}303 \\
(100.00 \%)\end{array}$ & - & & & - & - & $40(13.20 \%)$ & $\begin{array}{l}263 \\
(86.80 \%)\end{array}$ \\
\hline Rhys, Thomas & 2,820 & $\begin{array}{l}2621 \\
(92.94 \%)\end{array}$ & & & & & & $422(16.10 \%)$ & $\begin{array}{l}2199 \\
(83.90 \%)\end{array}$ & $\begin{array}{l}199 \\
(7.06 \%)\end{array}$ & & - & - & & & $44(22.11 \%)$ & $\begin{array}{l}155 \\
(77.89 \%)\end{array}$ \\
\hline Radon & 4,433 & $\begin{array}{l}4292 \\
(96.82 \%)\end{array}$ & $\begin{array}{l}711 \\
(16.57 \%)\end{array}$ & $\begin{array}{l}1194 \\
(27.82 \%)\end{array}$ & - & $\begin{array}{l}2144 \\
(49.95 \%)\end{array}$ & - & - & $\begin{array}{l}243 \\
(5.66 \%)\end{array}$ & $\begin{array}{l}141 \\
(3.18 \%)\end{array}$ & $\begin{array}{l}18 \\
(12.77 \%)\end{array}$ & $\begin{array}{l}40 \\
(28.37 \%)\end{array}$ & - & $\begin{array}{l}73 \\
(51.77 \%)\end{array}$ & - & & $\begin{array}{l}10 \\
(7.09 \%)\end{array}$ \\
\hline Hausfater & 1,062 & $\begin{array}{l}999 \\
(94.07 \%)\end{array}$ & $\begin{array}{l}246 \\
(24.62 \%)\end{array}$ & - & - & - & - & - & $\begin{array}{l}753 \\
(75.38 \%)\end{array}$ & $\begin{array}{l}61 \\
(5.74 \%)\end{array}$ & $4(6.56 \%)$ & & & - & - & - & $\begin{array}{l}57 \\
(93.44 \%)\end{array}$ \\
\hline Fernandez, Fuertes & 710 & $\begin{array}{l}664 \\
(93.52 \%)\end{array}$ & $\begin{array}{l}358 \\
(53.92 \%)\end{array}$ & - & - & - & & - & $\begin{array}{l}306 \\
(46.08 \%)\end{array}$ & $\begin{array}{l}46 \\
(6.48 \%)\end{array}$ & $\begin{array}{l}12 \\
(26.09 \%)\end{array}$ & & & - & - & - & $\begin{array}{l}34 \\
(73.91 \%)\end{array}$ \\
\hline Shields & 1,507 & $\begin{array}{l}1261 \\
(83.68 \%)\end{array}$ & $\begin{array}{l}145 \\
(11.50 \%)\end{array}$ & $\begin{array}{l}206 \\
(16.34 \%)\end{array}$ & & $\begin{array}{l}907 \\
(71.93 \%)\end{array}$ & - & & & $\begin{array}{l}246 \\
(16.32 \%)\end{array}$ & $12(4.88 \%)$ & $\begin{array}{l}41 \\
(16.67 \%)\end{array}$ & & $\begin{array}{l}193 \\
(78.46 \%)\end{array}$ & & & \\
\hline Gonzalez, Vielot & 782 & $\begin{array}{l}540 \\
(69.05 \%)\end{array}$ & & - & 75 (13.89\%) & $\begin{array}{l}465 \\
(86.11 \%)\end{array}$ & - & - & & $\begin{array}{l}242 \\
(30.95 \%)\end{array}$ & & - & $28(11.57 \%)$ & $\begin{array}{l}214 \\
(88.43 \%)\end{array}$ & - & & - \\
\hline Nielsen & 840 & $\begin{array}{l}630 \\
(75.00 \%)\end{array}$ & $29(4.60 \%)$ & $\begin{array}{l}204 \\
(32.38 \%)\end{array}$ & - & $\begin{array}{l}397 \\
(63.02 \%)\end{array}$ & - & - & & $\begin{array}{l}210 \\
(25.00 \%)\end{array}$ & $10(4.76 \%)$ & $\begin{array}{l}60 \\
(28.57 \%)\end{array}$ & - & $\begin{array}{l}140 \\
(66.67 \%)\end{array}$ & - & & - \\
\hline Jha & 8,967 & $\begin{array}{l}8799 \\
(98.13 \%)\end{array}$ & & - & $4120(46.82 \%)$ & $\begin{array}{l}4563 \\
(51.86 \%)\end{array}$ & - & & $\begin{array}{l}116 \\
(1.32 \%)\end{array}$ & $\begin{array}{l}168 \\
(1.87 \%)\end{array}$ & & - & $71(42.26 \%)$ & $\begin{array}{l}95 \\
(56.55 \%)\end{array}$ & & & $\begin{array}{l}2 \\
(1.19 \%)\end{array}$ \\
\hline Joubert, Andry & 1,013 & $\begin{array}{l}933 \\
(92.10 \%)\end{array}$ & $\begin{array}{l}158 \\
(16.93 \%)\end{array}$ & - & - & - & - & - & $\begin{array}{l}775 \\
(83.07 \%)\end{array}$ & $\begin{array}{l}80 \\
(7.90 \%)\end{array}$ & $4(5.00 \%)$ & & - & - & - & - & $\begin{array}{l}76 \\
(95.00 \%)\end{array}$ \\
\hline Daugherty & 266,586 & $\begin{array}{l}\text { NA( } \\
\text { NA\%) }\end{array}$ & & - & & - & - & & & $\begin{array}{l}266586 \\
(100.00 \%)\end{array}$ & $\begin{array}{l}8113 \\
(3.04 \%)\end{array}$ & - & - & & - & & $\begin{array}{l}258473 \\
(96.96 \%)\end{array}$ \\
\hline Vanegas, Cedillo & 551 & $\begin{array}{l}\text { NA ( } \\
\text { NA\%) }\end{array}$ & & - & - & - & - & - & - & $\begin{array}{l}551 \\
(100.00 \%)\end{array}$ & $27(4.90 \%)$ & - & - & & - & & $\begin{array}{l}524 \\
(95.10 \%)\end{array}$ \\
\hline Schaad & 134 & $\begin{array}{l}103 \\
(76.87 \%)\end{array}$ & $\begin{array}{l}32 \\
(31.07 \%)\end{array}$ & - & & - & - & & $\begin{array}{l}71 \\
(68.93 \%)\end{array}$ & $\begin{array}{l}31 \\
(23.13 \%)\end{array}$ & $7(22.58 \%)$ & - & - & & - & & $\begin{array}{l}24 \\
(77.42 \%)\end{array}$ \\
\hline Mok & 211 & $\begin{array}{l}\text { NA ( } \\
\text { NA\%) }\end{array}$ & & & & & & & & $\begin{array}{l}211 \\
(100.00 \%)\end{array}$ & & - & $78(36.97 \%)$ & & & & $\begin{array}{l}133 \\
(63.03 \%)\end{array}$ \\
\hline Desgranges & 507 & $\begin{array}{l}89 \\
(17.55 \%)\end{array}$ & $\begin{array}{l}27 \\
(30.34 \%)\end{array}$ & - & & - & - & & $\begin{array}{l}62 \\
(69.66 \%)\end{array}$ & $\begin{array}{l}418 \\
(82.45 \%)\end{array}$ & $\begin{array}{l}46 \\
(11.00 \%)\end{array}$ & - & - & & & & $\begin{array}{l}372 \\
(89.00 \%)\end{array}$ \\
\hline Gonzalez, Zepeda & 157 & $\begin{array}{l}97 \\
(61.78 \%)\end{array}$ & $\begin{array}{l}13 \\
(13.40 \%)\end{array}$ & - & & - & - & - & $\begin{array}{l}84 \\
(86.60 \%)\end{array}$ & $\begin{array}{l}60 \\
(38.22 \%)\end{array}$ & $5(8.33 \%)$ & - & - & & - & & $\begin{array}{l}55 \\
(91.67 \%)\end{array}$ \\
\hline Fogh & 3,236 & $\begin{array}{l}2632 \\
(81.33 \%)\end{array}$ & & - & $727(27.62 \%)$ & - & - & & $\begin{array}{l}1905 \\
(72.38 \%)\end{array}$ & $\begin{array}{l}604 \\
(18.67 \%)\end{array}$ & & - & $121(20.03 \%)$ & & - & & $\begin{array}{l}483 \\
(79.97 \%)\end{array}$ \\
\hline Rothlin, Eriksen & 819 & $\begin{array}{l}764 \\
(93.28 \%)\end{array}$ & & & $554(72.51 \%)$ & - & & & $\begin{array}{l}210 \\
(27.49 \%)\end{array}$ & $\begin{array}{l}55 \\
(6.72 \%)\end{array}$ & & - & $33(60.00 \%)$ & & & & $\begin{array}{l}22 \\
(40.00 \%)\end{array}$ \\
\hline Gornyk & 13,045 & $\begin{array}{l}12791 \\
(98.05 \%)\end{array}$ & $\begin{array}{l}2496 \\
(19.51 \%)\end{array}$ & $\begin{array}{l}3947 \\
(30.86 \%)\end{array}$ & & $\begin{array}{l}6335 \\
(49.53 \%)\end{array}$ & & & $\begin{array}{l}13 \\
(0.10 \%)\end{array}$ & $\begin{array}{l}254 \\
(1.95 \%)\end{array}$ & $\begin{array}{l}35 \\
(13.78 \%)\end{array}$ & $\begin{array}{l}84 \\
(33.07 \%)\end{array}$ & & $\begin{array}{l}135 \\
(53.15 \%)\end{array}$ & & & \\
\hline Campbell & 2,128 & $\begin{array}{l}2075 \\
(97.51 \%)\end{array}$ & $\begin{array}{l}326 \\
(15.71 \%)\end{array}$ & $\begin{array}{l}127 \\
(6.12 \%)\end{array}$ & - & $\begin{array}{l}1622 \\
(78.17 \%)\end{array}$ & - & - & & $\begin{array}{l}53 \\
(2.49 \%)\end{array}$ & $5(9.43 \%)$ & $2(3.77 \%)$ & - & $\begin{array}{l}46 \\
(86.79 \%)\end{array}$ & - & & - \\
\hline Kleynhans & 226 & $\begin{array}{l}129 \\
(57.08 \%)\end{array}$ & $\begin{array}{l}20 \\
(15.50 \%)\end{array}$ & & & - & - & & $\begin{array}{l}109 \\
(84.50 \%)\end{array}$ & $\begin{array}{l}97 \\
(42.92 \%)\end{array}$ & $\begin{array}{l}16 \\
(16.49 \%)\end{array}$ & - & - & & - & & $\begin{array}{l}81 \\
(83.51 \%)\end{array}$ \\
\hline Rogier & 374 & $\begin{array}{l}152 \\
(40.64 \%)\end{array}$ & $\begin{array}{l}28 \\
(18.42 \%)\end{array}$ & - & & - & - & & $\begin{array}{l}124 \\
(81.58 \%)\end{array}$ & $\begin{array}{l}222 \\
(59.36 \%)\end{array}$ & $8(3.60 \%)$ & - & - & & & & $\begin{array}{l}214 \\
(96.40 \%)\end{array}$ \\
\hline \multirow[t]{2}{*}{ Mostafa } & 4,040 & $\begin{array}{l}3770 \\
(93.32 \%)\end{array}$ & $\begin{array}{l}456 \\
(12.10 \%)\end{array}$ & 75 (1.99\%) & & $\begin{array}{l}3239 \\
(85.92 \%)\end{array}$ & & & & $\begin{array}{l}270 \\
(6.68 \%)\end{array}$ & $23(8.52 \%)$ & $4(1.48 \%)$ & & $\begin{array}{l}243 \\
(90.00 \%)\end{array}$ & & & \\
\hline & & & & & & & & & & & & & & & & & \\
\hline
\end{tabular}



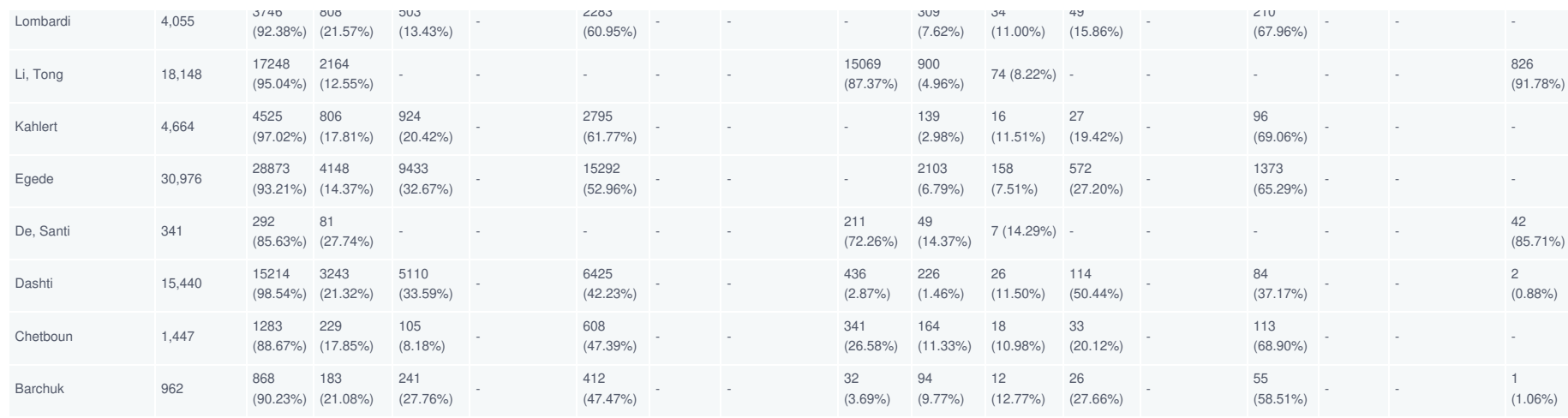

Forest plot of current smokers and risk of testing positive

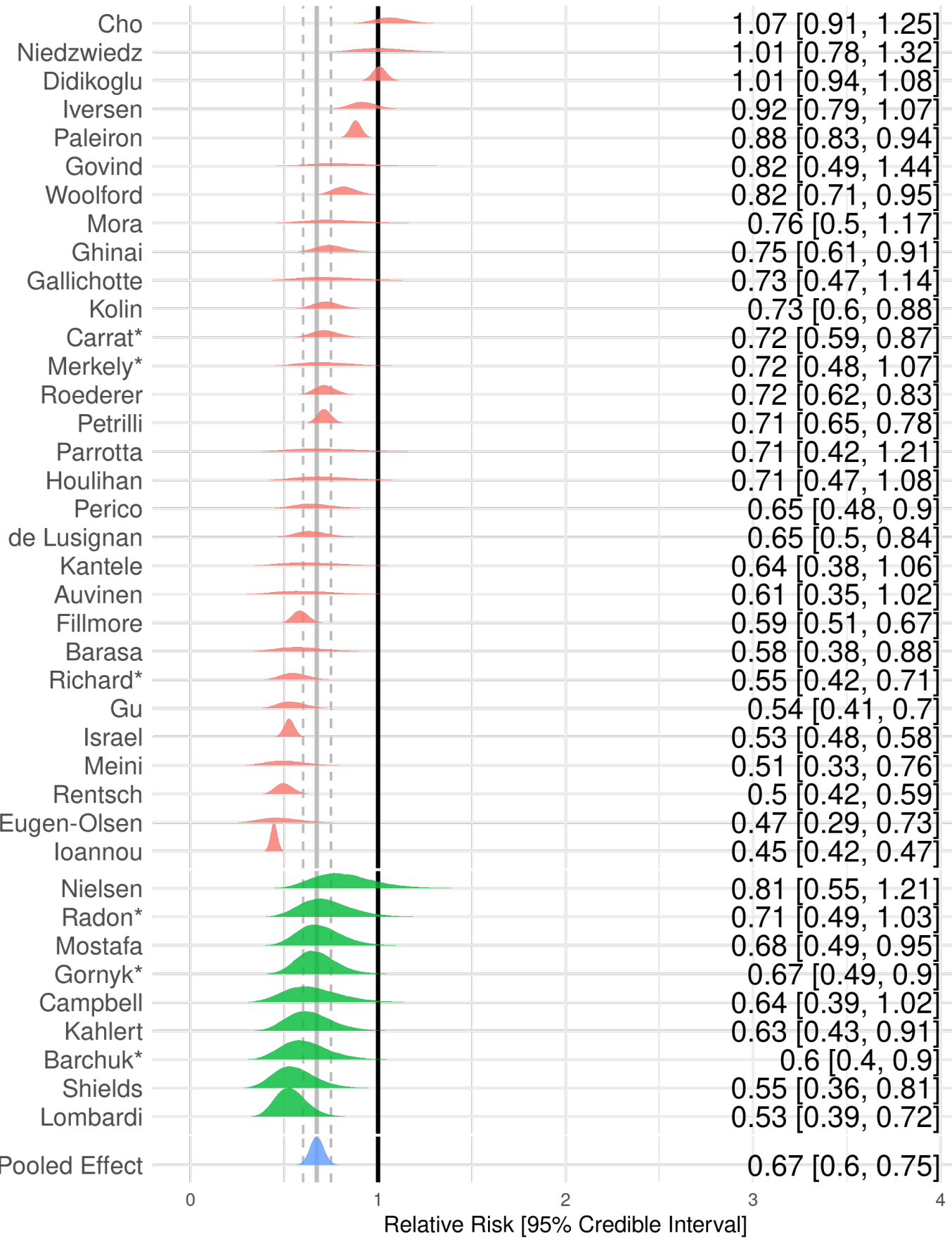

Review version

Previous version (v11)

Current version (v12)

Pooled effect 


\section{Forest plot of former smokers and risk of testing positive}

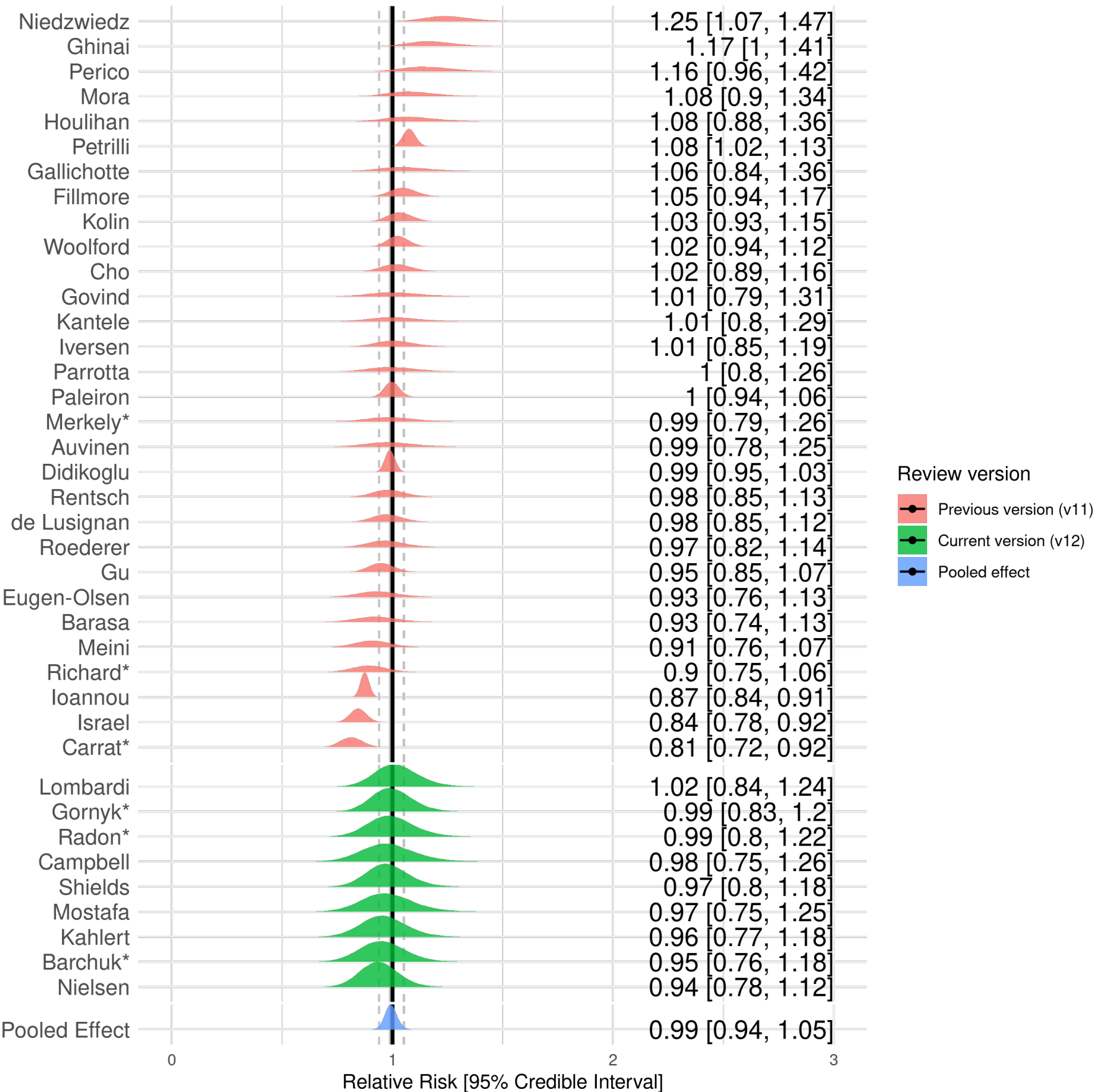

v11 testing prior

Table 3 COVID-19 hospitalisation by smoking status

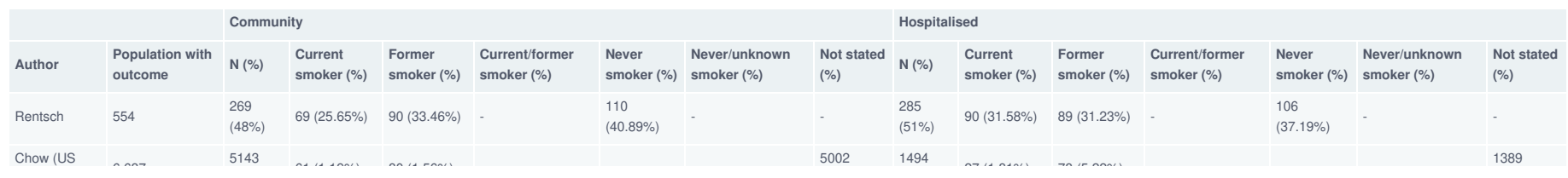




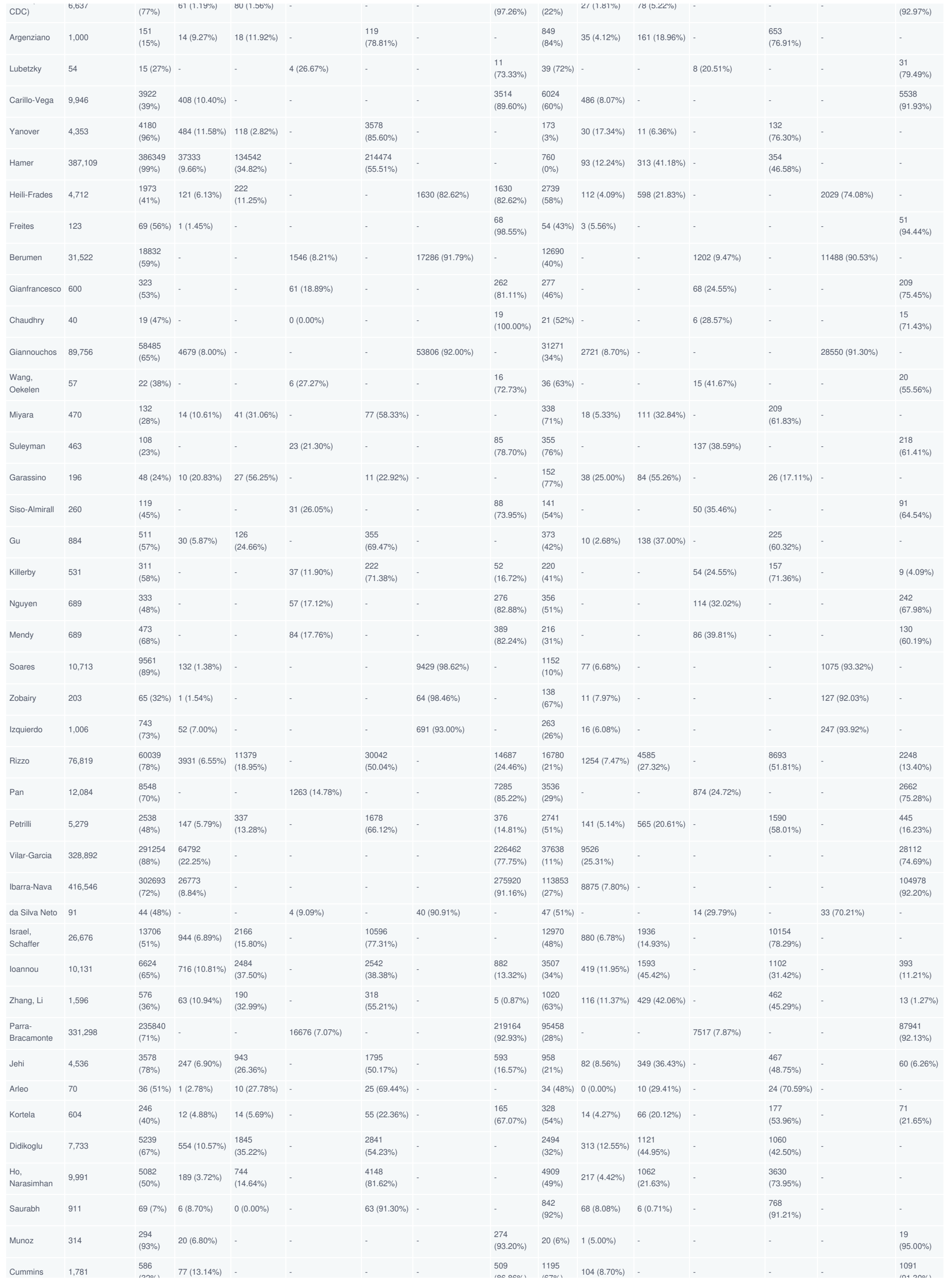




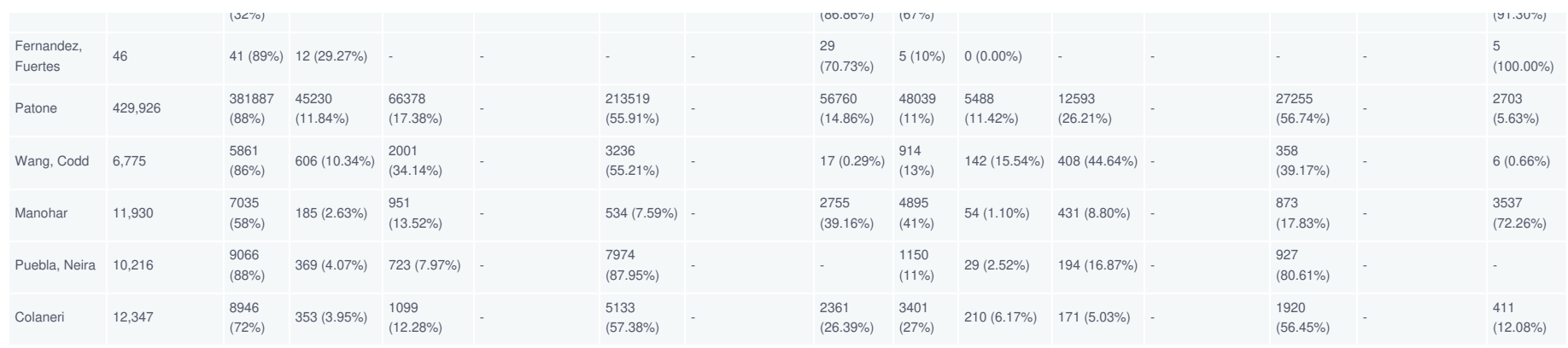

Forest plot of current smokers and risk of hospital admission

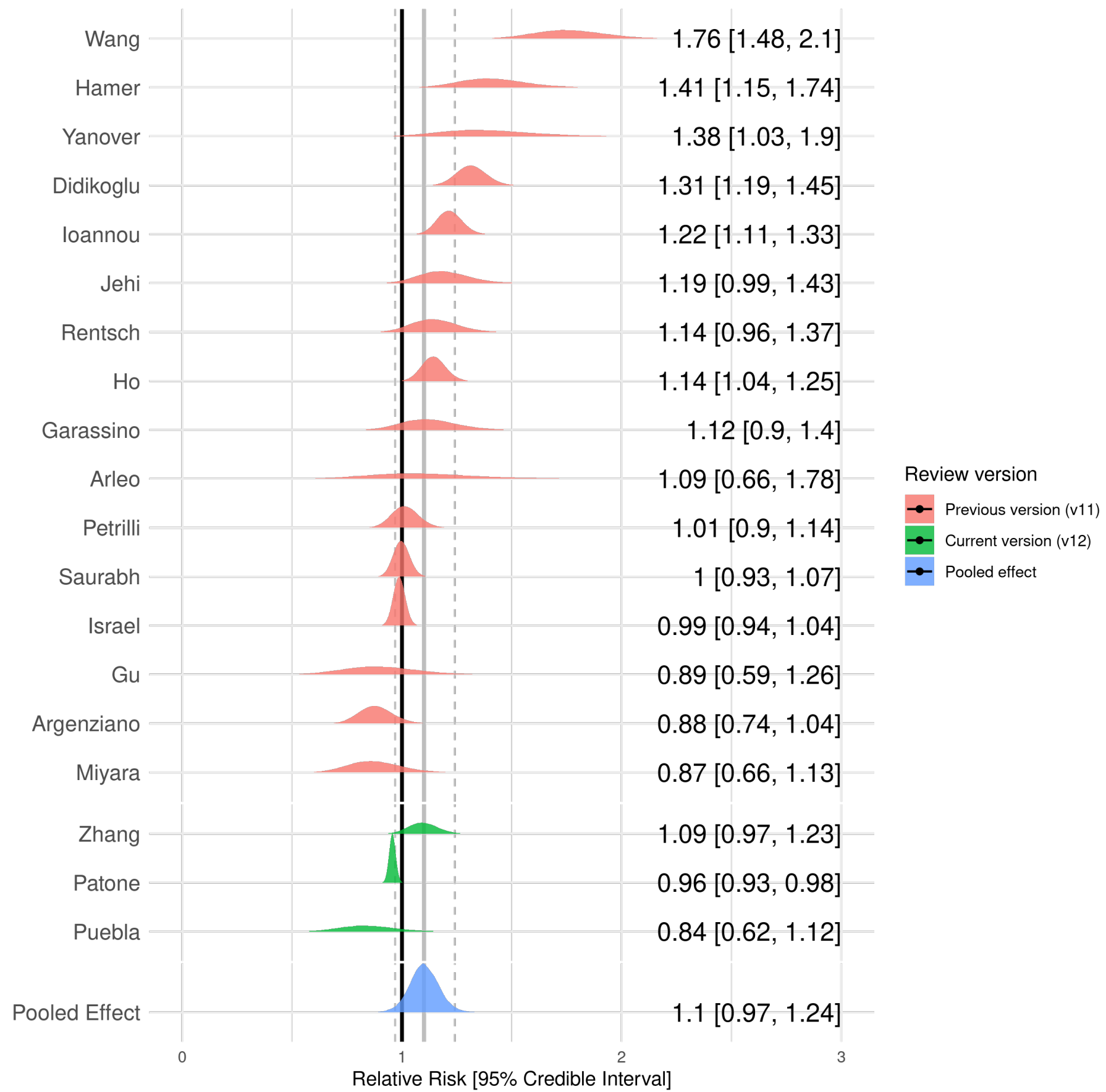

v11 hospitalisation prior 
Forest plot of former smokers and risk of hospital admission

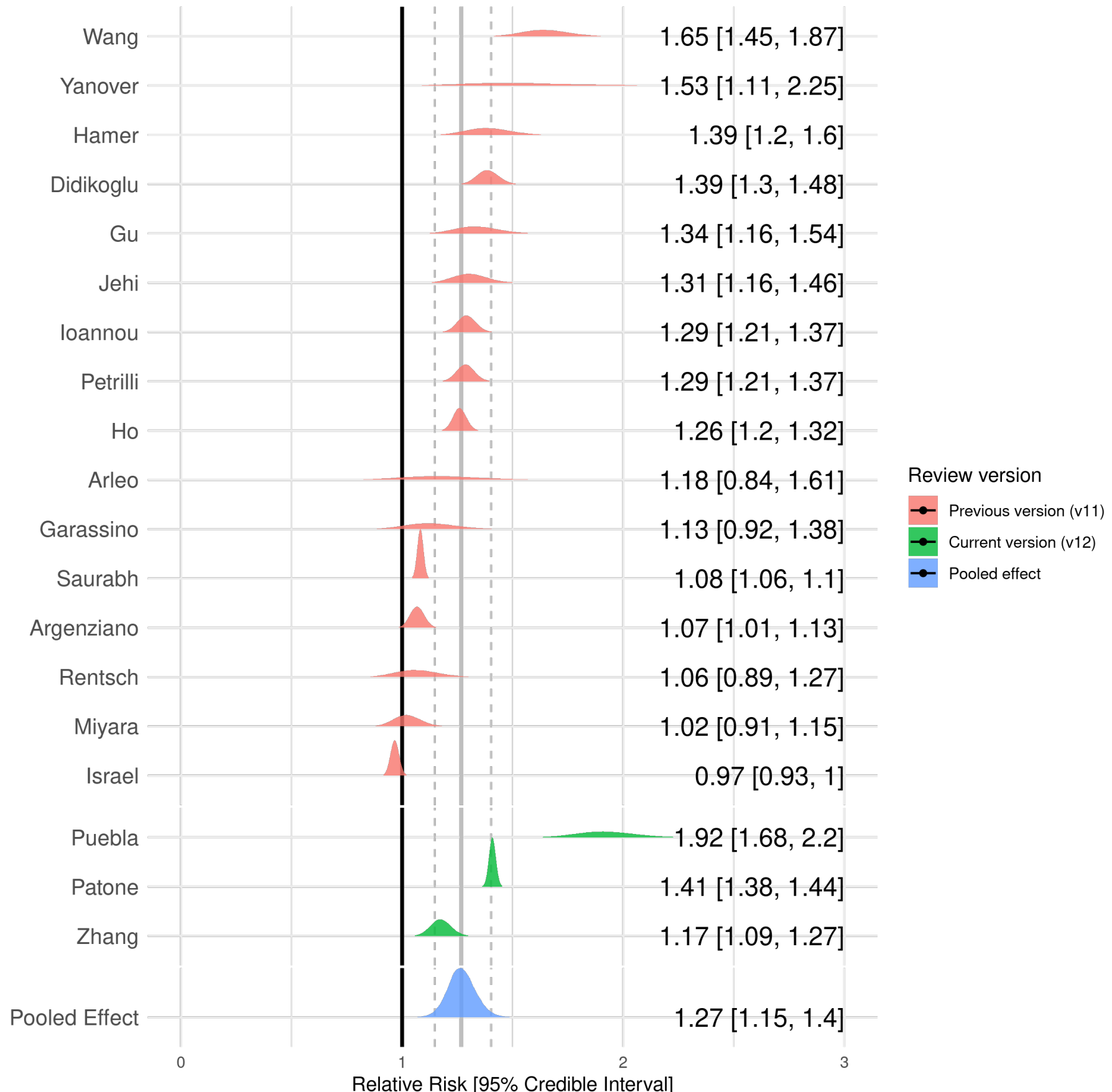

v11 hospitalisation prior

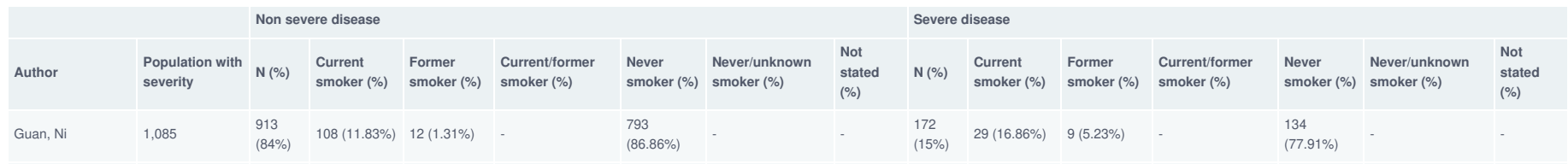




\begin{tabular}{|c|c|c|c|c|c|c|c|c|c|c|c|c|c|c|c|}
\hline Zhang, Dong & 9 & $3(33 \%)$ & $0(0.00 \%)$ & $3(100.00 \%)$ & & $0(0.00 \%)$ & - & & $6(66 \%)$ & $2(33.33 \%)$ & $4(66.67 \%)$ & - & $0(0.00 \%)$ & & - \\
\hline Wan & 9 & $8(88 \%)$ & $8(100.00 \%)$ & $0(0.00 \%)$ & & $0(0.00 \%)$ & & & $1(11 \%)$ & $1(100.00 \%)$ & $0(0.00 \%)$ & & $0(0.00 \%)$ & & \\
\hline Huang, Wang & 3 & $\begin{array}{l}3 \\
(100 \%)\end{array}$ & $3(100.00 \%)$ & $0(0.00 \%)$ & & $0(0.00 \%)$ & & & $0(0 \%)$ & 0 (NaN\%) & 0 (NaN\%) & & 0 (NaN\%) & & \\
\hline Rentsch & 285 & $\begin{array}{l}168 \\
(58 \%)\end{array}$ & $47(27.98 \%)$ & $53(31.55 \%)$ & & $68(40.48 \%)$ & & & $\begin{array}{l}117 \\
(41 \%)\end{array}$ & $43(36.75 \%)$ & $36(30.77 \%)$ & - & $38(32.48 \%)$ & & - \\
\hline $\mathrm{Hu}$ & 323 & $\begin{array}{l}151 \\
(46 \%)\end{array}$ & & & $12(7.95 \%)$ & & $139(92.05 \%)$ & & $\begin{array}{l}172 \\
(53 \%)\end{array}$ & & - & $26(15.12 \%)$ & & $146(84.88 \%)$ & - \\
\hline Wang, Pan & 125 & $\begin{array}{l}100 \\
(80 \%)\end{array}$ & - & & $9(9.00 \%)$ & - & $91(91.00 \%)$ & & $\begin{array}{l}25 \\
(20 \%)\end{array}$ & & - & $7(28.00 \%)$ & - & $18(72.00 \%)$ & - \\
\hline Kim & 27 & $\begin{array}{l}21 \\
(77 \%)\end{array}$ & $3(14.29 \%)$ & & & & $18(85.71 \%)$ & & $6(22 \%)$ & $2(33.33 \%)$ & $0(0.00 \%)$ & - & & $4(66.67 \%)$ & \\
\hline Shi, Yu & 474 & $\begin{array}{l}425 \\
(89 \%)\end{array}$ & & & $34(8.00 \%)$ & & $391(92.00 \%)$ & & $\begin{array}{l}49 \\
(10 \%)\end{array}$ & & & $6(12.24 \%)$ & & $43(87.76 \%)$ & \\
\hline Liao, Feng & 148 & $\begin{array}{l}92 \\
(62 \%)\end{array}$ & & & $5(5.43 \%)$ & & & $\begin{array}{l}87 \\
(94.57 \%)\end{array}$ & $\begin{array}{l}56 \\
(37 \%)\end{array}$ & $3(5.36 \%)$ & & - & & & $\begin{array}{l}53 \\
(94.64 \%)\end{array}$ \\
\hline Shi, Ren & 134 & $\begin{array}{l}88 \\
(65 \%)\end{array}$ & & & $8(9.09 \%)$ & & & $\begin{array}{l}80 \\
(90.91 \%)\end{array}$ & $\begin{array}{l}46 \\
(34 \%)\end{array}$ & & & $6(13.04 \%)$ & & & $\begin{array}{l}40 \\
(86.96 \%)\end{array}$ \\
\hline Hadjadj & 50 & $\begin{array}{l}15 \\
(30 \%)\end{array}$ & $1(6.67 \%)$ & $2(13.33 \%)$ & & $12(80.00 \%)$ & & & $\begin{array}{l}35 \\
(70 \%)\end{array}$ & $0(0.00 \%)$ & $7(20.00 \%)$ & & $28(80.00 \%)$ & & \\
\hline Zheng, Xiong & 73 & $\begin{array}{l}43 \\
\text { (58\%) }\end{array}$ & & & $6(13.95 \%)$ & $37(86.05 \%)$ & - & & $\begin{array}{l}30 \\
(41 \%)\end{array}$ & & & $2(6.67 \%)$ & $28(93.33 \%)$ & & \\
\hline de la Rica & 48 & $\begin{array}{l}26 \\
(54 \%)\end{array}$ & & & $6(23.08 \%)$ & & & $\begin{array}{l}20 \\
(76.92 \%)\end{array}$ & $\begin{array}{l}20 \\
(41 \%)\end{array}$ & & & $4(20.00 \%)$ & & & $\begin{array}{l}16 \\
(80.00 \%)\end{array}$ \\
\hline Yin, Yang & 106 & $\begin{array}{l}47 \\
(44 \%)\end{array}$ & & & $6(12.77 \%)$ & & & $\begin{array}{l}41 \\
(87.23 \%)\end{array}$ & $\begin{array}{l}59 \\
(55 \%)\end{array}$ & & & $12(20.34 \%)$ & & & $\begin{array}{l}47 \\
(79.66 \%)\end{array}$ \\
\hline Allenbach & 147 & $\begin{array}{l}100 \\
(68 \%)\end{array}$ & & & $9(9.00 \%)$ & & & $\begin{array}{l}91 \\
(91.00 \%)\end{array}$ & $\begin{array}{l}47 \\
(31 \%)\end{array}$ & & & $0(0.00 \%)$ & & & $\begin{array}{l}47 \\
(100.00 \%)\end{array}$ \\
\hline Goyal & 393 & $\begin{array}{l}263 \\
(66 \%)\end{array}$ & $14(5.32 \%)$ & & & - & & $\begin{array}{l}249 \\
(94.68 \%)\end{array}$ & $\begin{array}{l}130 \\
(33 \%)\end{array}$ & $6(4.62 \%)$ & & - & & & $\begin{array}{l}124 \\
(95.38 \%)\end{array}$ \\
\hline Feng & 454 & $\begin{array}{l}333 \\
(73 \%)\end{array}$ & $27(8.11 \%)$ & & & & & $\begin{array}{l}306 \\
(91.89 \%)\end{array}$ & $\begin{array}{l}121 \\
(26 \%)\end{array}$ & $17(14.05 \%)$ & - & - & & & $\begin{array}{l}104 \\
(85.95 \%)\end{array}$ \\
\hline Yao & 108 & $\begin{array}{l}83 \\
(76 \%)\end{array}$ & $1(1.20 \%)$ & & & & & $\begin{array}{l}82 \\
(98.80 \%)\end{array}$ & $\begin{array}{l}25 \\
(23 \%)\end{array}$ & $3(12.00 \%)$ & & - & & & $\begin{array}{l}22 \\
(88.00 \%)\end{array}$ \\
\hline Regina & 200 & $\begin{array}{l}163 \\
(81 \%)\end{array}$ & $9(5.52 \%)$ & & & - & & $\begin{array}{l}154 \\
(94.48 \%)\end{array}$ & $\begin{array}{l}37 \\
(18 \%)\end{array}$ & $0(0.00 \%)$ & & & & & $\begin{array}{l}37 \\
(100.00 \%)\end{array}$ \\
\hline Feuth & 28 & $\begin{array}{l}21 \\
(75 \%)\end{array}$ & $1(4.76 \%)$ & $7(33.33 \%)$ & & $13(61.90 \%)$ & & & $7(25 \%)$ & $2(28.57 \%)$ & $1(14.29 \%)$ & & $4(57.14 \%)$ & & \\
\hline Mejia-Vilet & 329 & $\begin{array}{l}214 \\
(65 \%)\end{array}$ & & & $13(6.07 \%)$ & & & $\begin{array}{l}201 \\
(93.93 \%)\end{array}$ & $\begin{array}{l}115 \\
(34 \%)\end{array}$ & & & $10(8.70 \%)$ & & & $\begin{array}{l}105 \\
(91.30 \%)\end{array}$ \\
\hline Chen, Jiang & 135 & $\begin{array}{l}54 \\
(40 \%)\end{array}$ & & & $4(7.41 \%)$ & - & - & $\begin{array}{l}50 \\
(92.59 \%)\end{array}$ & $\begin{array}{l}81 \\
(60 \%)\end{array}$ & & - & $9(11.11 \%)$ & - & - & $\begin{array}{l}72 \\
(88.89 \%)\end{array}$ \\
\hline Vaquero-Roncero & 146 & $\begin{array}{l}75 \\
(51 \%)\end{array}$ & & & $4(5.33 \%)$ & - & - & $\begin{array}{l}71 \\
(94.67 \%)\end{array}$ & $\begin{array}{l}71 \\
(48 \%)\end{array}$ & & - & $6(8.45 \%)$ & . & & $\begin{array}{l}65 \\
(91.55 \%)\end{array}$ \\
\hline Kim, Garg & 2,490 & $\begin{array}{l}1692 \\
(67 \%)\end{array}$ & $112(6.62 \%)$ & $\begin{array}{l}395 \\
(23.35 \%)\end{array}$ & & & 1185 (70.04\%) & & $\begin{array}{l}798 \\
(32 \%)\end{array}$ & $38(4.76 \%)$ & $\begin{array}{l}247 \\
(30.95 \%)\end{array}$ & & & $512(64.16 \%)$ & \\
\hline Wu & 174 & $\begin{array}{l}92 \\
(52 \%)\end{array}$ & & & $47(51.09 \%)$ & & $45(48.91 \%)$ & & $\begin{array}{l}82 \\
(47 \%)\end{array}$ & $11(13.41 \%)$ & & & & 71 (86.59\%) & \\
\hline Chaudhry & 40 & $\begin{array}{l}34 \\
(85 \%)\end{array}$ & & & $5(14.71 \%)$ & & & $\begin{array}{l}29 \\
(85.29 \%)\end{array}$ & $6(15 \%)$ & & & $1(16.67 \%)$ & & & $\begin{array}{l}5 \\
(83.33 \%)\end{array}$ \\
\hline Garibaldi & 832 & $\begin{array}{l}532 \\
(63 \%)\end{array}$ & $25(4.70 \%)$ & $\begin{array}{l}107 \\
(20.11 \%)\end{array}$ & & - & - & $\begin{array}{l}400 \\
(75.19 \%)\end{array}$ & $\begin{array}{l}300 \\
(36 \%)\end{array}$ & $21(7.00 \%)$ & $81(27.00 \%)$ & - & & & $\begin{array}{l}198 \\
(66.00 \%)\end{array}$ \\
\hline Kuderer & 928 & $\begin{array}{l}686 \\
(73 \%)\end{array}$ & $35(5.10 \%)$ & $\begin{array}{l}210 \\
(30.61 \%)\end{array}$ & & $\begin{array}{l}370 \\
(53.94 \%)\end{array}$ & - & $\begin{array}{l}29 \\
(4.23 \%)\end{array}$ & $\begin{array}{l}242 \\
(26 \%)\end{array}$ & $8(3.31 \%)$ & $\begin{array}{l}116 \\
(47.93 \%)\end{array}$ & - & 99 (40.91\%) & $15(6.20 \%)$ & $4(1.65 \%)$ \\
\hline Romao & 14 & $\begin{array}{l}14 \\
(100 \%)\end{array}$ & & & $4(28.57 \%)$ & & & $\begin{array}{l}10 \\
(71.43 \%)\end{array}$ & $0(0 \%)$ & & - & - & & & \\
\hline Giannouchos & 89,756 & $\begin{array}{l}78050 \\
(86 \%)\end{array}$ & $6322(8.10 \%)$ & & & & 71728 (91.90\%) & & $\begin{array}{l}11706 \\
(13 \%)\end{array}$ & $1089(9.30 \%)$ & & & & $10617(90.70 \%)$ & \\
\hline Cen & 1,007 & $\begin{array}{l}720 \\
(71 \%)\end{array}$ & & & $70(9.72 \%)$ & & & $\begin{array}{l}650 \\
(90.28 \%)\end{array}$ & $\begin{array}{l}287 \\
(28 \%)\end{array}$ & & & $18(6.27 \%)$ & & & $\begin{array}{l}269 \\
(93.73 \%)\end{array}$ \\
\hline Maraschini & 132 & $\begin{array}{l}89 \\
(67 \%)\end{array}$ & & $11(12.36 \%)$ & & $78(87.64 \%)$ & & & $\begin{array}{l}43 \\
(32 \%)\end{array}$ & & $3(6.98 \%)$ & & 40 (93.02\%) & & \\
\hline Russell & 156 & $\begin{array}{l}128 \\
(82 \%)\end{array}$ & $9(7.03 \%)$ & $31(24.22 \%)$ & - & 51 (39.84\%) & - & $\begin{array}{l}37 \\
(28.91 \%)\end{array}$ & $\begin{array}{l}28 \\
(17 \%)\end{array}$ & $2(7.14 \%)$ & $8(28.57 \%)$ & - & $8(28.57 \%)$ & - & $\begin{array}{l}10 \\
(35.71 \%)\end{array}$ \\
\hline Siso-Almirall & 260 & $\begin{array}{l}212 \\
(81 \%)\end{array}$ & - & & $60(28.30 \%)$ & - & - & $\begin{array}{l}152 \\
(71.70 \%)\end{array}$ & $\begin{array}{l}48 \\
(18 \%)\end{array}$ & - & - & $21(43.75 \%)$ & - & & $\begin{array}{l}27 \\
(56.25 \%)\end{array}$ \\
\hline Gu & 645 & $\begin{array}{l}511 \\
(79 \%)\end{array}$ & $30(5.87 \%)$ & $\begin{array}{l}126 \\
(24.66 \%)\end{array}$ & & $\begin{array}{l}355 \\
(69.47 \%)\end{array}$ & & & $\begin{array}{l}134 \\
(20 \%)\end{array}$ & $3(2.24 \%)$ & $61(45.52 \%)$ & - & 70 (52.24\%) & & - \\
\hline Mendy & 689 & $\begin{array}{l}598 \\
(86 \%)\end{array}$ & & & $133(22.24 \%)$ & & & $\begin{array}{l}465 \\
(77.76 \%)\end{array}$ & $\begin{array}{l}91 \\
(13 \%)\end{array}$ & & & $37(40.66 \%)$ & & & $\begin{array}{l}54 \\
(59.34 \%)\end{array}$ \\
\hline Jin, Gu & 6 & $2(33 \%)$ & & & $0(0.00 \%)$ & & & $\begin{array}{l}2 \\
(100.00 \%)\end{array}$ & $4(66 \%)$ & & & $2(50.00 \%)$ & & & $\begin{array}{l}2 \\
(50.00 \%)\end{array}$ \\
\hline Senkal & 611 & $\begin{array}{l}446 \\
(73 \%)\end{array}$ & $48(10.76 \%)$ & & & - & - & $\begin{array}{l}398 \\
(89.24 \%)\end{array}$ & $\begin{array}{l}165 \\
(27 \%)\end{array}$ & $21(12.73 \%)$ & - & - & & & $\begin{array}{l}144 \\
(87.27 \%)\end{array}$ \\
\hline Patel & 129 & $\begin{array}{l}89 \\
(68 \%)\end{array}$ & $26(29.21 \%)$ & & & - & $58(65.17 \%)$ & $5(5.62 \%)$ & $\begin{array}{l}40 \\
(31 \%)\end{array}$ & $22(55.00 \%)$ & - & & & $14(35.00 \%)$ & $\begin{array}{l}4 \\
(10.00 \%)\end{array}$ \\
\hline Maucourant & 27 & $\begin{array}{l}10 \\
(37 \%)\end{array}$ & $1(10.00 \%)$ & $2(20.00 \%)$ & & $2(20.00 \%)$ & & $\begin{array}{l}5 \\
(50.00 \%)\end{array}$ & $\begin{array}{l}17 \\
(62 \%)\end{array}$ & $2(11.76 \%)$ & $5(29.41 \%)$ & - & 9 (52.94\%) & & $1(5.88 \%)$ \\
\hline Xie & 619 & $\begin{array}{l}469 \\
(75 \%)\end{array}$ & & & $32(6.82 \%)$ & & & $\begin{array}{l}437 \\
(93.18 \%)\end{array}$ & $\begin{array}{l}150 \\
(24 \%)\end{array}$ & & & $19(12.67 \%)$ & & & $\begin{array}{l}131 \\
(87.33 \%)\end{array}$ \\
\hline
\end{tabular}




\begin{tabular}{|c|c|c|c|c|c|c|c|c|c|c|c|c|c|c|c|}
\hline Fox & 55 & $(54 \%)$ & $1(3.33 \%)$ & $4(13.33 \%)$ & - & 17 (56.67\%) & - & (26.67\%) & $\begin{array}{l}<0 \\
(45 \%)\end{array}$ & $0(0.00 \%)$ & $2(8.00 \%)$ & - & $14(56.00 \%)$ & - & $(36.00 \%)$ \\
\hline Zhang, Cao & 240 & $\begin{array}{l}162 \\
(67 \%)\end{array}$ & $2(1.23 \%)$ & $6(3.70 \%)$ & - & - & & $\begin{array}{l}154 \\
(95.06 \%)\end{array}$ & $\begin{array}{l}78 \\
(32 \%)\end{array}$ & $4(5.13 \%)$ & $4(5.13 \%)$ & - & - & - & $\begin{array}{l}70 \\
(89.74 \%)\end{array}$ \\
\hline Kurashima & 53 & $\begin{array}{l}10 \\
(18 \%)\end{array}$ & & - & $3(30.00 \%)$ & - & & $\begin{array}{l}7 \\
(70.00 \%)\end{array}$ & $\begin{array}{l}43 \\
(81 \%)\end{array}$ & - & - & $24(55.81 \%)$ & - & - & $\begin{array}{l}19 \\
(44.19 \%)\end{array}$ \\
\hline Zhan & 75 & $\begin{array}{l}\text { NA } \\
(\mathrm{NA} \%)\end{array}$ & & - & - & - & & - & $\begin{array}{l}75 \\
(100 \%)\end{array}$ & - & - & $9(12.00 \%)$ & - & - & $\begin{array}{l}66 \\
(88.00 \%)\end{array}$ \\
\hline Omrani & 858 & $\begin{array}{l}806 \\
(93 \%)\end{array}$ & - & - & $121(15.01 \%)$ & - & - & $\begin{array}{l}685 \\
(84.99 \%)\end{array}$ & $52(6 \%)$ & & - & $9(17.31 \%)$ & - & - & $\begin{array}{l}43 \\
(82.69 \%)\end{array}$ \\
\hline Marcos & 918 & $\begin{array}{l}555 \\
(60 \%)\end{array}$ & $38(6.85 \%)$ & - & $69(12.43 \%)$ & - & - & $\begin{array}{l}448 \\
(80.72 \%)\end{array}$ & $\begin{array}{l}363 \\
(39 \%)\end{array}$ & $18(4.96 \%)$ & - & $71(19.56 \%)$ & - & - & $\begin{array}{l}292 \\
(80.44 \%)\end{array}$ \\
\hline $\begin{array}{l}\text { Hoertel, Sanchez, } \\
\text { Rico }\end{array}$ & 7,345 & $\begin{array}{l}6014 \\
(81 \%)\end{array}$ & $433(7.20 \%)$ & - & & - & - & $\begin{array}{l}5581 \\
(92.80 \%)\end{array}$ & $\begin{array}{l}1331 \\
(18 \%)\end{array}$ & $190(14.27 \%)$ & - & - & - & - & $\begin{array}{l}1141 \\
(85.73 \%)\end{array}$ \\
\hline Qi & 267 & $\begin{array}{l}217 \\
(81 \%)\end{array}$ & $22(10.14 \%)$ & - & & - & $195(89.86 \%)$ & & $\begin{array}{l}50 \\
(18 \%)\end{array}$ & $31(62.00 \%)$ & - & - & - & $19(38.00 \%)$ & \\
\hline Monteiro & 112 & $\begin{array}{l}84 \\
(75 \%)\end{array}$ & $3(3.57 \%)$ & $14(16.67 \%)$ & & $63(75.00 \%)$ & - & $4(4.76 \%)$ & $\begin{array}{l}28 \\
(25 \%)\end{array}$ & $4(14.29 \%)$ & $6(21.43 \%)$ & - & $14(50.00 \%)$ & - & $\begin{array}{l}4 \\
(14.29 \%)\end{array}$ \\
\hline Morshed & 103 & $\begin{array}{l}87 \\
(84 \%)\end{array}$ & $28(32.18 \%)$ & & & & $59(67.82 \%)$ & & $\begin{array}{l}16 \\
(15 \%)\end{array}$ & $4(25.00 \%)$ & & & - & $12(75.00 \%)$ & \\
\hline Zhou, Sun & 144 & $\begin{array}{l}108 \\
(75 \%)\end{array}$ & $11(10.19 \%)$ & & & & & $\begin{array}{l}97 \\
(89.81 \%)\end{array}$ & $\begin{array}{l}36 \\
(25 \%)\end{array}$ & $2(5.56 \%)$ & & & & & $\begin{array}{l}34 \\
(94.44 \%)\end{array}$ \\
\hline Zhao, Chen & 641 & $\begin{array}{l}398 \\
(62 \%)\end{array}$ & $87(21.86 \%)$ & & & & & $\begin{array}{l}311 \\
(78.14 \%)\end{array}$ & $\begin{array}{l}195 \\
(30 \%)\end{array}$ & $52(26.67 \%)$ & & & & & $\begin{array}{l}143 \\
(73.33 \%)\end{array}$ \\
\hline Qu & 246 & $\begin{array}{l}226 \\
(91 \%)\end{array}$ & $90(39.82 \%)$ & & & & & $\begin{array}{l}136 \\
(60.18 \%)\end{array}$ & $20(8 \%)$ & $14(70.00 \%)$ & & & & & $\begin{array}{l}6 \\
(30.00 \%)\end{array}$ \\
\hline Petrilli & 2,729 & $\begin{array}{l}1739 \\
(63 \%)\end{array}$ & $97(5.58 \%)$ & $\begin{array}{l}325 \\
(18.69 \%)\end{array}$ & & $\begin{array}{l}1067 \\
(61.36 \%)\end{array}$ & & $\begin{array}{l}250 \\
(14.38 \%)\end{array}$ & $\begin{array}{l}990 \\
(36 \%)\end{array}$ & $44(4.44 \%)$ & $\begin{array}{l}236 \\
(23.84 \%)\end{array}$ & & $\begin{array}{l}517 \\
(52.22 \%)\end{array}$ & & $\begin{array}{l}193 \\
(19.49 \%)\end{array}$ \\
\hline Ren & 432 & $\begin{array}{l}314 \\
(72 \%)\end{array}$ & $26(8.28 \%)$ & - & - & $\begin{array}{l}288 \\
(91.72 \%)\end{array}$ & - & - & $\begin{array}{l}118 \\
(27 \%)\end{array}$ & $17(14.41 \%)$ & - & - & $\begin{array}{l}101 \\
(85.59 \%)\end{array}$ & - & - \\
\hline Yan & 578 & $\begin{array}{l}450 \\
(77 \%)\end{array}$ & $31(6.89 \%)$ & $\cdot$ & - & - & & $\begin{array}{l}419 \\
(93.11 \%)\end{array}$ & $\begin{array}{l}128 \\
(22 \%)\end{array}$ & 20 (15.62\%) & - & - & - & - & $\begin{array}{l}108 \\
(84.38 \%)\end{array}$ \\
\hline Nicholson & 1,042 & $\begin{array}{l}550 \\
(52 \%)\end{array}$ & $37(6.73 \%)$ & $\begin{array}{l}106 \\
(19.27 \%)\end{array}$ & - & $\begin{array}{l}211 \\
(38.36 \%)\end{array}$ & & $\begin{array}{l}196 \\
(35.64 \%)\end{array}$ & $\begin{array}{l}401 \\
(38 \%)\end{array}$ & $41(10.22 \%)$ & $92(22.94 \%)$ & - & $\begin{array}{l}155 \\
(38.65 \%)\end{array}$ & - & $\begin{array}{l}113 \\
(28.18 \%)\end{array}$ \\
\hline Zhu & 432 & $\begin{array}{l}285 \\
(65 \%)\end{array}$ & $46(16.14 \%)$ & - & - & - & & $\begin{array}{l}239 \\
(83.86 \%)\end{array}$ & $\begin{array}{l}147 \\
(34 \%)\end{array}$ & $16(10.88 \%)$ & - & - & - & - & $\begin{array}{l}147 \\
(100.00 \%)\end{array}$ \\
\hline Kalan & 193 & $\begin{array}{l}122 \\
(63 \%)\end{array}$ & $9(7.38 \%)$ & - & - & $\begin{array}{l}102 \\
(83.61 \%)\end{array}$ & & $\begin{array}{l}11 \\
(9.02 \%)\end{array}$ & $\begin{array}{l}71 \\
(36 \%)\end{array}$ & $5(7.04 \%)$ & - & - & $62(87.32 \%)$ & - & $4(5.63 \%)$ \\
\hline Burrell & 204 & $\begin{array}{l}85 \\
(41 \%)\end{array}$ & - & $\cdot$ & $7(8.24 \%)$ & - & $75(88.24 \%)$ & $3(3.53 \%)$ & $\begin{array}{l}119 \\
(58 \%)\end{array}$ & & - & $20(16.81 \%)$ & - & 94 (78.99\%) & $5(4.20 \%)$ \\
\hline Zhou, He, Yang & 1,087 & $\begin{array}{l}990 \\
(91 \%)\end{array}$ & - & - & $849(85.76 \%)$ & $\begin{array}{l}141 \\
(14.24 \%)\end{array}$ & - & & $97(8 \%)$ & & - & $75(77.32 \%)$ & $22(22.68 \%)$ & - & \\
\hline Zhou, Qin & 51 & $\begin{array}{l}\text { NA } \\
(\mathrm{NA} \%)\end{array}$ & - & - & & - & - & & $\begin{array}{l}51 \\
(100 \%)\end{array}$ & & - & $40(78.43 \%)$ & $11(21.57 \%)$ & - & \\
\hline Zhan, Liu & 405 & $\begin{array}{l}257 \\
(63 \%)\end{array}$ & - & - & $21(8.17 \%)$ & $\begin{array}{l}236 \\
(91.83 \%)\end{array}$ & - & & $\begin{array}{l}148 \\
(36 \%)\end{array}$ & & - & $25(16.89 \%)$ & $\begin{array}{l}123 \\
(83.11 \%)\end{array}$ & - & - \\
\hline Li, Long, Zhang & 954 & $\begin{array}{l}838 \\
(87 \%)\end{array}$ & - & - & $34(4.06 \%)$ & $\begin{array}{l}804 \\
(95.94 \%)\end{array}$ & - & - & $\begin{array}{l}116 \\
(12 \%)\end{array}$ & & - & $22(18.97 \%)$ & 94 (81.03\%) & - & - \\
\hline Jakob & 2,155 & $\begin{array}{l}1400 \\
(64 \%)\end{array}$ & & & $92(6.57 \%)$ & $99(7.07 \%)$ & & $\begin{array}{l}669 \\
(47.79 \%)\end{array}$ & $\begin{array}{l}755 \\
(35 \%)\end{array}$ & $51(6.75 \%)$ & $58(7.68 \%)$ & & $\begin{array}{l}200 \\
(26.49 \%)\end{array}$ & & $\begin{array}{l}446 \\
(59.07 \%)\end{array}$ \\
\hline Aksu & 123 & $\begin{array}{l}34 \\
(27 \%)\end{array}$ & $3(8.82 \%)$ & & & & $31(91.18 \%)$ & & $\begin{array}{l}89 \\
(72 \%)\end{array}$ & $11(12.36 \%)$ & & & & $78(87.64 \%)$ & \\
\hline $\begin{array}{l}\text { Hoertel, Sanchez, } \\
\text { Vernet }\end{array}$ & 12,210 & $\begin{array}{l}11018 \\
(90 \%)\end{array}$ & $921(8.36 \%)$ & & & & & $\begin{array}{l}10097 \\
(91.64 \%)\end{array}$ & $\begin{array}{l}1192 \\
(9 \%)\end{array}$ & $181(15.18 \%)$ & & & - & & $\begin{array}{l}1011 \\
(84.82 \%)\end{array}$ \\
\hline $\begin{array}{l}\text { Vila-Corcoles, } \\
\text { Satue-Gracia }\end{array}$ & 282 & $\begin{array}{l}218 \\
(77 \%)\end{array}$ & & & $21(9.63 \%)$ & & & $\begin{array}{l}197 \\
(90.37 \%)\end{array}$ & $\begin{array}{l}64 \\
(22 \%)\end{array}$ & & & $4(6.25 \%)$ & & & $\begin{array}{l}60 \\
(93.75 \%)\end{array}$ \\
\hline Boyd & 38 & $0(0 \%)$ & & & & & & & $\begin{array}{l}38 \\
(100 \%)\end{array}$ & & & $2(5.26 \%)$ & & & $\begin{array}{l}36 \\
(94.74 \%)\end{array}$ \\
\hline Caliskan & 565 & $\begin{array}{l}474 \\
(83 \%)\end{array}$ & $96(20.25 \%)$ & $45(9.49 \%)$ & & $\begin{array}{l}333 \\
(70.25 \%)\end{array}$ & - & & $\begin{array}{l}91 \\
(16 \%)\end{array}$ & $22(24.18 \%)$ & $34(37.36 \%)$ & - & $35(38.46 \%)$ & - & \\
\hline Ebrahimian & 226 & $\begin{array}{l}134 \\
(59 \%)\end{array}$ & - & - & $12(8.96 \%)$ & - & $122(91.04 \%)$ & - & $\begin{array}{l}92 \\
(40 \%)\end{array}$ & - & - & $12(13.04 \%)$ & - & $80(86.96 \%)$ & - \\
\hline Ho, Narasimhan & 4,909 & $\begin{array}{l}3859 \\
(78 \%)\end{array}$ & $169(4.38 \%)$ & $\begin{array}{l}833 \\
(21.59 \%)\end{array}$ & - & - & 2857 (74.03\%) & - & $\begin{array}{l}1050 \\
(21 \%)\end{array}$ & $8(4.57 \%)$ & $\begin{array}{l}229 \\
(21.81 \%)\end{array}$ & - & $\begin{array}{l}773 \\
(73.62 \%)\end{array}$ & - & - \\
\hline Quan & 2,038 & $\begin{array}{l}1452 \\
(71 \%)\end{array}$ & - & $\begin{array}{l}549 \\
(37.81 \%)\end{array}$ & - & - & & $\begin{array}{l}903 \\
(62.19 \%)\end{array}$ & $\begin{array}{l}586 \\
(28 \%)\end{array}$ & - & - & $261(44.54 \%)$ & - & - & $\begin{array}{l}325 \\
(55.46 \%)\end{array}$ \\
\hline Saurabh & 911 & $\begin{array}{l}783 \\
(85 \%)\end{array}$ & $65(8.30 \%)$ & $3(0.38 \%)$ & - & $\begin{array}{l}715 \\
(91.32 \%)\end{array}$ & & - & $\begin{array}{l}128 \\
(14 \%)\end{array}$ & $9(7.03 \%)$ & $3(2.34 \%)$ & - & $\begin{array}{l}116 \\
(90.62 \%)\end{array}$ & - & - \\
\hline Chousein & 114 & $\begin{array}{l}94 \\
(82 \%)\end{array}$ & 17 (18.09\%) & $17(18.09 \%)$ & & $60(63.83 \%)$ & - & & $\begin{array}{l}20 \\
(17 \%)\end{array}$ & $2(10.00 \%)$ & $6(30.00 \%)$ & - & $12(60.00 \%)$ & - & - \\
\hline Tavakol & 206 & $\begin{array}{l}182 \\
(88 \%)\end{array}$ & - & - & 24 (13.19\%) & - & $158(86.81 \%)$ & & $\begin{array}{l}24 \\
(11 \%)\end{array}$ & & - & $2(8.33 \%)$ & - & $22(91.67 \%)$ & - \\
\hline Yao, Hasegawa & 101 & $\begin{array}{l}78 \\
(77 \%)\end{array}$ & - & - & $17(21.79 \%)$ & - & $71(91.03 \%)$ & & $\begin{array}{l}23 \\
(22 \%)\end{array}$ & & - & $12(52.17 \%)$ & - & $11(47.83 \%)$ & \\
\hline Cummins & 1,195 & $\begin{array}{l}1043 \\
(87 \%)\end{array}$ & $96(9.20 \%)$ & - & & - & - & $\begin{array}{l}947 \\
(90.80 \%)\end{array}$ & $\begin{array}{l}152 \\
(12 \%)\end{array}$ & $9(5.92 \%)$ & - & - & - & - & $\begin{array}{l}143 \\
(94.08 \%)\end{array}$ \\
\hline Nuno & 4,730 & $\begin{array}{l}3536 \\
(74 \%)\end{array}$ & $333(9.42 \%)$ & - & & - & - & $\begin{array}{l}3203 \\
(90.58 \%)\end{array}$ & $\begin{array}{l}1194 \\
(25 \%)\end{array}$ & $147(12.31 \%)$ & & - & - & - & $\begin{array}{l}1047 \\
(87.69 \%)\end{array}$ \\
\hline $\begin{array}{l}\text { P-HOSP-COVID } \\
\text { Collaborative }\end{array}$ & 1,077 & $\begin{array}{l}604 \\
(56 \%)\end{array}$ & $11(1.82 \%)$ & $\begin{array}{l}205 \\
(33.94 \%)\end{array}$ & & $\begin{array}{l}277 \\
(45.86 \%)\end{array}$ & & $\begin{array}{l}111 \\
(18.38 \%)\end{array}$ & $\begin{array}{l}473 \\
(43 \%)\end{array}$ & $5(1.06 \%)$ & $\begin{array}{l}165 \\
(34.88 \%)\end{array}$ & & $\begin{array}{l}228 \\
(48.20 \%)\end{array}$ & & $\begin{array}{l}75 \\
(15.86 \%)\end{array}$ \\
\hline Faverio & 312 & $\begin{array}{l}71 \\
(22 \%)\end{array}$ & $1(1.41 \%)$ & $8(11.27 \%)$ & & $47(66.20 \%)$ & & $\begin{array}{l}15 \\
(21.13 \%)\end{array}$ & $\begin{array}{l}241 \\
(77 \%)\end{array}$ & $14(5.81 \%)$ & $57(23.65 \%)$ & & $\begin{array}{l}134 \\
(55.60 \%)\end{array}$ & & $\begin{array}{l}36 \\
(14.94 \%)\end{array}$ \\
\hline
\end{tabular}




\begin{tabular}{|c|c|c|c|c|c|c|c|c|c|c|c|c|c|c|c|}
\hline Manohar & 4,895 & $\begin{array}{l}3825 \\
(78 \%)\end{array}$ & 41 (1.07\%) & $288(7.53 \%)$ & & $\begin{array}{l}647 \\
(16.92 \%)\end{array}$ & & $\begin{array}{l}2849 \\
(74.48 \%)\end{array}$ & $\begin{array}{l}1070 \\
(21 \%)\end{array}$ & $13(1.21 \%)$ & $\begin{array}{l}143 \\
(13.36 \%)\end{array}$ & - & $\begin{array}{l}226 \\
(21.12 \%)\end{array}$ & - & $\begin{array}{l}688 \\
(64.30 \%)\end{array}$ \\
\hline Jha, Shrestha & 198 & $\begin{array}{l}165 \\
(83 \%)\end{array}$ & $17(10.30 \%)$ & $19(11.52 \%)$ & & $\begin{array}{l}129 \\
(78.18 \%)\end{array}$ & & & $\begin{array}{l}33 \\
(16 \%)\end{array}$ & $6(18.18 \%)$ & $6(18.18 \%)$ & - & 21 (63.64\%) & - & - \\
\hline Riou & 124 & $\begin{array}{l}90 \\
(72 \%)\end{array}$ & - & - & 34 (37.78\%) & - & & $\begin{array}{l}56 \\
(62.22 \%)\end{array}$ & $\begin{array}{l}34 \\
(27 \%)\end{array}$ & - & - & $17(50.00 \%)$ & . & - & $\begin{array}{l}17 \\
(50.00 \%)\end{array}$ \\
\hline Rachmawati & 490 & $\begin{array}{l}472 \\
(96 \%)\end{array}$ & $76(16.10 \%)$ & $61(12.92 \%)$ & & $\begin{array}{l}335 \\
(70.97 \%)\end{array}$ & & & $18(3 \%)$ & $8(44.44 \%)$ & $3(16.67 \%)$ & - & $7(38.89 \%)$ & - & - \\
\hline Peng, Lei & 622 & $\begin{array}{l}254 \\
(40 \%)\end{array}$ & $19(7.48 \%)$ & - & - & & & $\begin{array}{l}235 \\
(92.52 \%)\end{array}$ & $\begin{array}{l}368 \\
(59 \%)\end{array}$ & $43(11.68 \%)$ & - & - & . & - & $\begin{array}{l}325 \\
(88.32 \%)\end{array}$ \\
\hline Hojbjerg, Lassen & 171 & $\begin{array}{l}127 \\
(74 \%)\end{array}$ & $10(7.87 \%)$ & $58(45.67 \%)$ & & $56(44.09 \%)$ & & $3(2.36 \%)$ & $\begin{array}{l}44 \\
(25 \%)\end{array}$ & 0 (0.00\%) & $34(77.27 \%)$ & & $10(22.73 \%)$ & & \\
\hline Alsafar & 873 & $\begin{array}{l}574 \\
(65 \%)\end{array}$ & $77(13.41 \%)$ & & & & & $\begin{array}{l}497 \\
(86.59 \%)\end{array}$ & $\begin{array}{l}299 \\
(34 \%)\end{array}$ & $60(20.07 \%)$ & - & & & & $\begin{array}{l}239 \\
(79.93 \%)\end{array}$ \\
\hline Ali, Hasan & 464 & $\begin{array}{l}319 \\
(68 \%)\end{array}$ & $47(14.73 \%)$ & - & & & 272 (85.27\%) & & $\begin{array}{l}145 \\
(31 \%)\end{array}$ & $3(2.07 \%)$ & - & - & - & $142(97.93 \%)$ & - \\
\hline
\end{tabular}

\section{Forest plot of current smokers and risk of severe disease}

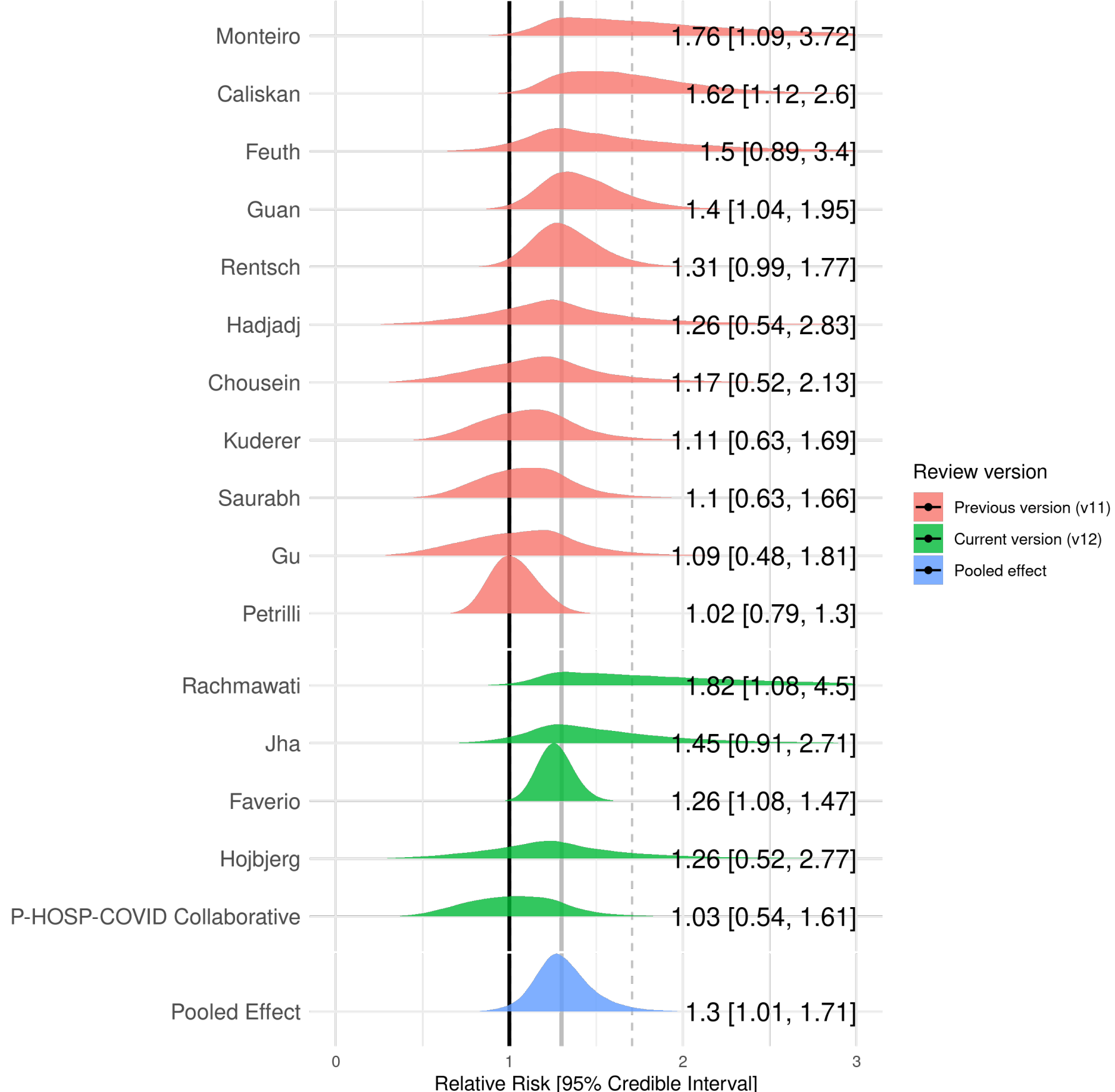

Relative Risk [95\% Credible Interval] 


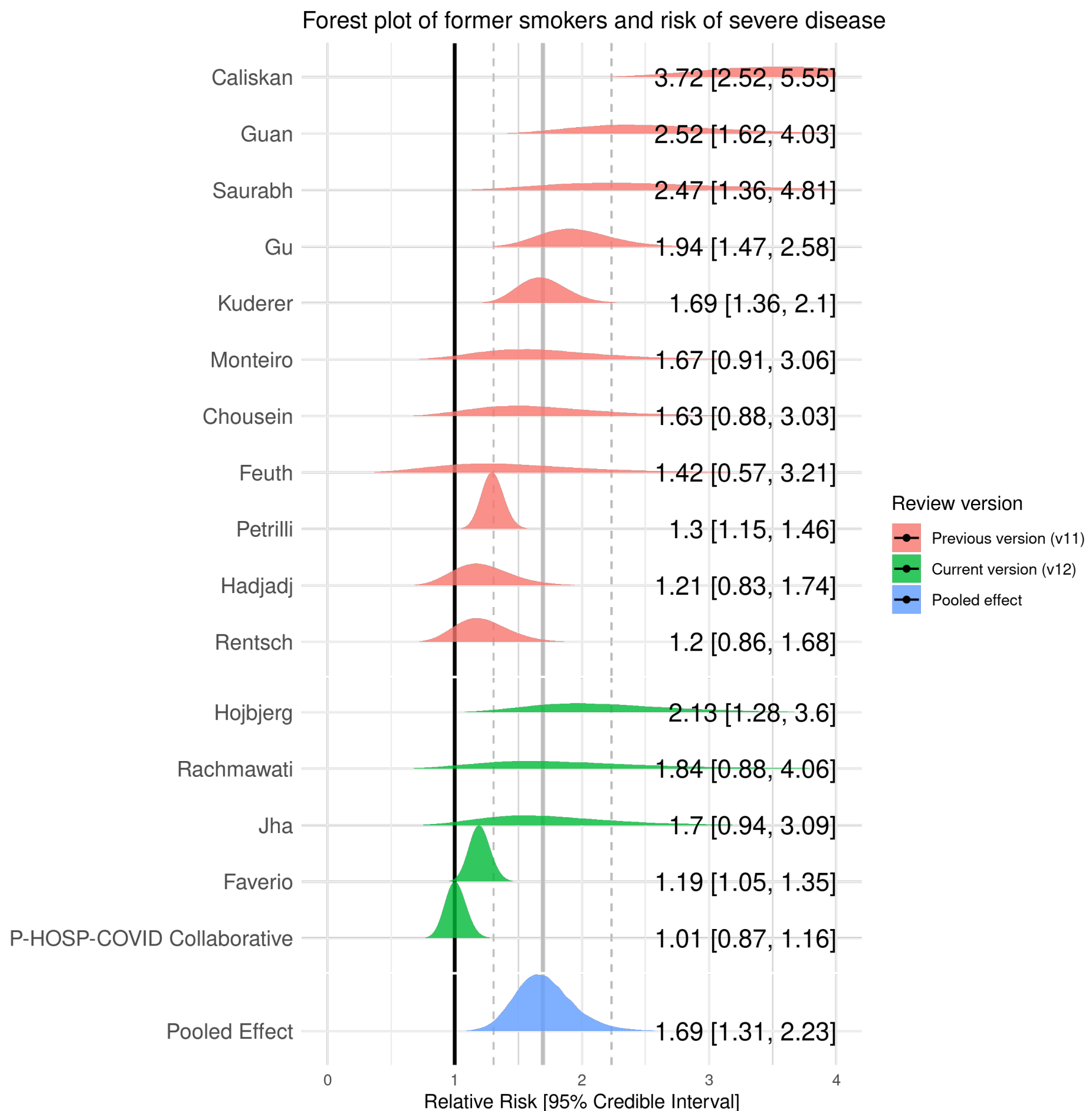

v11 severity prior 


\begin{tabular}{|c|c|c|c|c|c|c|c|c|c|c|c|c|c|c|c|}
\hline & & 100101 & & & & & & (W6.15010) & (ד) & & & & & & (WC.vT/o) \\
\hline Zhou, Yu & 191 & $\begin{array}{l}137 \\
(71 \%)\end{array}$ & $6(4.38 \%)$ & & - & & & $\begin{array}{l}131 \\
(95.62 \%)\end{array}$ & $\begin{array}{l}54 \\
(28 \%)\end{array}$ & $5(9.26 \%)$ & & - & - & - & $\begin{array}{l}49 \\
(90.74 \%)\end{array}$ \\
\hline Yang, Yu & 52 & $\begin{array}{l}20 \\
(38 \%)\end{array}$ & $2(10.00 \%)$ & & - & - & $18(90.00 \%)$ & - & $\begin{array}{l}32 \\
(61 \%)\end{array}$ & - & & - & - & $32(100.00 \%)$ & - \\
\hline Borobia & 2,226 & $\begin{array}{l}1766 \\
(79 \%)\end{array}$ & $113(6.40 \%)$ & & - & & & $\begin{array}{l}1653 \\
(93.60 \%)\end{array}$ & $\begin{array}{l}460 \\
(20 \%)\end{array}$ & $44(9.57 \%)$ & & - & - & & $\begin{array}{l}416 \\
(90.43 \%)\end{array}$ \\
\hline Giacomelli & 233 & $\begin{array}{l}185 \\
(79 \%)\end{array}$ & & & $53(28.65 \%)$ & $\begin{array}{l}132 \\
(71.35 \%)\end{array}$ & & & $\begin{array}{l}48 \\
(20 \%)\end{array}$ & & & $17(35.42 \%)$ & $31(64.58 \%)$ & & $0(0.00 \%)$ \\
\hline Yao & 108 & $\begin{array}{l}96 \\
(88 \%)\end{array}$ & $1(1.04 \%)$ & & & - & & $\begin{array}{l}95 \\
(98.96 \%)\end{array}$ & $\begin{array}{l}12 \\
(11 \%)\end{array}$ & $3(25.00 \%)$ & & - & - & & $9(75.00 \%)$ \\
\hline Carillo-Vega & 9,946 & $\begin{array}{l}8983 \\
(90 \%)\end{array}$ & $795(8.85 \%)$ & & & & & $\begin{array}{l}8188 \\
(91.15 \%)\end{array}$ & $\begin{array}{l}963 \\
(9 \%)\end{array}$ & $99(10.28 \%)$ & & & - & & $\begin{array}{l}864 \\
(89.72 \%)\end{array}$ \\
\hline Ge & 51 & $\begin{array}{l}39 \\
(76 \%)\end{array}$ & $6(15.38 \%)$ & & - & & & $\begin{array}{l}33 \\
(84.62 \%)\end{array}$ & $\begin{array}{l}12 \\
(23 \%)\end{array}$ & $1(8.33 \%)$ & & & - & & $\begin{array}{l}11 \\
(91.67 \%)\end{array}$ \\
\hline Chen, Jiang & 135 & $\begin{array}{l}\text { NA } \\
(\mathrm{NA} \%)\end{array}$ & - & & & & & & $\begin{array}{l}31 \\
(22 \%)\end{array}$ & - & & $4(12.90 \%)$ & & & $\begin{array}{l}27 \\
(87.10 \%)\end{array}$ \\
\hline Heili-Frades & 4,712 & $\begin{array}{l}4086 \\
(86 \%)\end{array}$ & $210(5.14 \%)$ & $\begin{array}{l}659 \\
(16.13 \%)\end{array}$ & - & & $3217(78.73 \%)$ & - & $\begin{array}{l}626 \\
(13 \%)\end{array}$ & $23(3.67 \%)$ & $\begin{array}{l}161 \\
(25.72 \%)\end{array}$ & - & - & $442(70.61 \%)$ & - \\
\hline Kim, Garg & 2,490 & $\begin{array}{l}2070 \\
(83 \%)\end{array}$ & $128(6.18 \%)$ & $\begin{array}{l}481 \\
(23.24 \%)\end{array}$ & - & - & $1461(70.58 \%)$ & - & $\begin{array}{l}420 \\
(16 \%)\end{array}$ & $22(5.24 \%)$ & $\begin{array}{l}161 \\
(38.33 \%)\end{array}$ & - & - & $236(56.19 \%)$ & - \\
\hline Al-Hindawi & 31 & $\begin{array}{l}15 \\
(48 \%)\end{array}$ & $0(0.00 \%)$ & $10(66.67 \%)$ & - & $5(33.33 \%)$ & & - & $\begin{array}{l}16 \\
(51 \%)\end{array}$ & $1(6.25 \%)$ & $12(75.00 \%)$ & - & $3(18.75 \%)$ & & - \\
\hline Louis & 22 & $\begin{array}{l}16 \\
(72 \%)\end{array}$ & - & & $7(43.75 \%)$ & & & $\begin{array}{l}9 \\
(56.25 \%)\end{array}$ & $6(27 \%)$ & - & & $3(50.00 \%)$ & - & & $3(50.00 \%)$ \\
\hline Soto-Mota & 400 & $\begin{array}{l}200 \\
(50 \%)\end{array}$ & - & & $23(11.50 \%)$ & & & $\begin{array}{l}177 \\
(88.50 \%)\end{array}$ & $\begin{array}{l}200 \\
(50 \%)\end{array}$ & - & & $25(12.50 \%)$ & - & - & $\begin{array}{l}175 \\
(87.50 \%)\end{array}$ \\
\hline Garibaldi & 747 & $\begin{array}{l}634 \\
(84 \%)\end{array}$ & $36(5.68 \%)$ & $\begin{array}{l}129 \\
(20.35 \%)\end{array}$ & & & & $\begin{array}{l}469 \\
(73.97 \%)\end{array}$ & $\begin{array}{l}113 \\
(15 \%)\end{array}$ & $6(5.31 \%)$ & $36(31.86 \%)$ & & - & & $\begin{array}{l}71 \\
(62.83 \%)\end{array}$ \\
\hline Docherty & 13,364 & $\begin{array}{l}8199 \\
(61 \%)\end{array}$ & $370(4.51 \%)$ & $\begin{array}{l}1832 \\
(22.34 \%)\end{array}$ & & $\begin{array}{l}4179 \\
(50.97 \%)\end{array}$ & & $\begin{array}{l}1818 \\
(22.17 \%)\end{array}$ & $\begin{array}{l}5165 \\
(38 \%)\end{array}$ & $214(4.14 \%)$ & $\begin{array}{l}1350 \\
(26.14 \%)\end{array}$ & & $\begin{array}{l}2105 \\
(40.76 \%)\end{array}$ & & $\begin{array}{l}1496 \\
(28.96 \%)\end{array}$ \\
\hline Kuderer & 928 & $\begin{array}{l}807 \\
(86 \%)\end{array}$ & $38(4.71 \%)$ & $\begin{array}{l}262 \\
(32.47 \%)\end{array}$ & & $\begin{array}{l}425 \\
(52.66 \%)\end{array}$ & & $31(3.84 \%)$ & $\begin{array}{l}121 \\
(13 \%)\end{array}$ & $5(4.13 \%)$ & $64(52.89 \%)$ & & $44(36.36 \%)$ & & $2(1.65 \%)$ \\
\hline Ramlall & 11,116 & $\begin{array}{l}10498 \\
(94 \%)\end{array}$ & - & & $2771(26.40 \%)$ & $\begin{array}{l}7727 \\
(73.60 \%)\end{array}$ & & - & $\begin{array}{l}618 \\
(5 \%)\end{array}$ & & & $208(33.66 \%)$ & $\begin{array}{l}410 \\
(66.34 \%)\end{array}$ & & - \\
\hline Wang, Oekelen & 57 & $\begin{array}{l}43 \\
(75 \%)\end{array}$ & & & $14(32.56 \%)$ & & & $\begin{array}{l}29 \\
(67.44 \%)\end{array}$ & $\begin{array}{l}14 \\
(24 \%)\end{array}$ & & & $7(50.00 \%)$ & - & & $7(50.00 \%)$ \\
\hline Martinez-Portilla & 224 & $\begin{array}{l}217 \\
(96 \%)\end{array}$ & - & & $7(3.23 \%)$ & & & $\begin{array}{l}210 \\
(96.77 \%)\end{array}$ & $7(3 \%)$ & - & & $0(0.00 \%)$ & - & & $\begin{array}{l}7 \\
(100.00 \%)\end{array}$ \\
\hline Cen & 1,007 & $\begin{array}{l}964 \\
(95 \%)\end{array}$ & - & & $87(9.02 \%)$ & - & & $\begin{array}{l}877 \\
(90.98 \%)\end{array}$ & $43(4 \%)$ & - & & $1(2.33 \%)$ & - & & $\begin{array}{l}42 \\
(97.67 \%)\end{array}$ \\
\hline Klang & 3,406 & $\begin{array}{l}2270 \\
(66 \%)\end{array}$ & - & & $492(21.67 \%)$ & & & $\begin{array}{l}1778 \\
(78.33 \%)\end{array}$ & $\begin{array}{l}1136 \\
(33 \%)\end{array}$ & - & & $301(26.50 \%)$ & - & - & $\begin{array}{l}835 \\
(73.50 \%)\end{array}$ \\
\hline Wang, Zhong & 5,510 & $\begin{array}{l}4874 \\
(88 \%)\end{array}$ & 247 (5.07\%) & $\begin{array}{l}1083 \\
(22.22 \%)\end{array}$ & - & $\begin{array}{l}3544 \\
(72.71 \%)\end{array}$ & & - & $\begin{array}{l}636 \\
(11 \%)\end{array}$ & $28(4.40 \%)$ & $\begin{array}{l}214 \\
(33.65 \%)\end{array}$ & - & $\begin{array}{l}394 \\
(61.95 \%)\end{array}$ & - & - \\
\hline Miyara & 338 & $\begin{array}{l}211 \\
(62 \%)\end{array}$ & $13(6.16 \%)$ & $58(27.49 \%)$ & - & $\begin{array}{l}141 \\
(66.82 \%)\end{array}$ & & - & $\begin{array}{l}46 \\
(13 \%)\end{array}$ & $1(2.17 \%)$ & $23(50.00 \%)$ & - & $21(45.65 \%)$ & & - \\
\hline Rajter & 255 & $\begin{array}{l}209 \\
(81 \%)\end{array}$ & & & $28(13.40 \%)$ & $\begin{array}{l}181 \\
(86.60 \%)\end{array}$ & & - & $\begin{array}{l}53 \\
(20 \%)\end{array}$ & & & $18(33.96 \%)$ & $28(52.83 \%)$ & & - \\
\hline Zeng & 1,031 & $\begin{array}{l}866 \\
(84 \%)\end{array}$ & & & 69 (7.97\%) & & & $\begin{array}{l}797 \\
(92.03 \%)\end{array}$ & $\begin{array}{l}165 \\
(16 \%)\end{array}$ & & & $36(21.82 \%)$ & - & & $\begin{array}{l}129 \\
(78.18 \%)\end{array}$ \\
\hline Chen, Yu & 1,859 & $\begin{array}{l}1651 \\
(88 \%)\end{array}$ & $32(1.94 \%)$ & $54(3.27 \%)$ & & $\begin{array}{l}1565 \\
(94.79 \%)\end{array}$ & & & $\begin{array}{l}208 \\
(11 \%)\end{array}$ & $13(6.25 \%)$ & 12 (5.77\%) & & $\begin{array}{l}183 \\
(87.98 \%)\end{array}$ & & \\
\hline Garassino & 190 & $\begin{array}{l}124 \\
(65 \%)\end{array}$ & & & $92(74.19 \%)$ & $\begin{array}{l}32 \\
(25.81 \%)\end{array}$ & & & $\begin{array}{l}66 \\
(34 \%)\end{array}$ & & $61(92.42 \%)$ & & $5(7.58 \%)$ & & - \\
\hline Gu & 884 & $\begin{array}{l}864 \\
(97 \%)\end{array}$ & $40(4.63 \%)$ & $\begin{array}{l}250 \\
(28.94 \%)\end{array}$ & & $\begin{array}{l}219 \\
(25.35 \%)\end{array}$ & & & $20(2 \%)$ & $0(0.00 \%)$ & $14(70.00 \%)$ & & $6(30.00 \%)$ & & - \\
\hline Sigel & 88 & $\begin{array}{l}70 \\
(79 \%)\end{array}$ & & & $37(52.86 \%)$ & & & $\begin{array}{l}33 \\
(47.14 \%)\end{array}$ & $\begin{array}{l}18 \\
(20 \%)\end{array}$ & & & $11(61.11 \%)$ & - & & $7(38.89 \%)$ \\
\hline Nguyen & 356 & $\begin{array}{l}308 \\
(86 \%)\end{array}$ & - & & $91(29.55 \%)$ & & & $\begin{array}{l}217 \\
(70.45 \%)\end{array}$ & $\begin{array}{l}45 \\
(12 \%)\end{array}$ & - & & $23(51.11 \%)$ & - & & $\begin{array}{l}22 \\
(48.89 \%)\end{array}$ \\
\hline de Souza & 8,443 & $\begin{array}{l}7826 \\
(92 \%)\end{array}$ & - & & $95(1.21 \%)$ & & 7571 (96.74\%) & $\begin{array}{l}160 \\
(2.04 \%)\end{array}$ & $\begin{array}{l}617 \\
(7 \%)\end{array}$ & - & & $47(7.62 \%)$ & - & $560(90.76 \%)$ & $10(1.62 \%)$ \\
\hline Mendy & 689 & $\begin{array}{l}663 \\
(96 \%)\end{array}$ & - & & $160(24.13 \%)$ & & & $\begin{array}{l}502 \\
(75.72 \%)\end{array}$ & $26(3 \%)$ & - & & $10(38.46 \%)$ & - & & $\begin{array}{l}16 \\
(61.54 \%)\end{array}$ \\
\hline Shi, Resurreccion & 256 & $\begin{array}{l}210 \\
(82 \%)\end{array}$ & - & & $128(60.95 \%)$ & & & $\begin{array}{l}82 \\
(39.05 \%)\end{array}$ & $\begin{array}{l}46 \\
(17 \%)\end{array}$ & - & & $26(56.52 \%)$ & - & - & $\begin{array}{l}20 \\
(43.48 \%)\end{array}$ \\
\hline Xie & 619 & $\begin{array}{l}591 \\
(95 \%)\end{array}$ & & & $43(7.28 \%)$ & & & $\begin{array}{l}548 \\
(92.72 \%)\end{array}$ & $28(4 \%)$ & & & $8(28.57 \%)$ & & & $\begin{array}{l}20 \\
(71.43 \%)\end{array}$ \\
\hline Fox & 54 & $\begin{array}{l}35 \\
(64 \%)\end{array}$ & $1(2.86 \%)$ & $4(11.43 \%)$ & & $\begin{array}{l}18 \\
(51.43 \%)\end{array}$ & & $\begin{array}{l}12 \\
(34.29 \%)\end{array}$ & $\begin{array}{l}19 \\
(35 \%)\end{array}$ & $0(0.00 \%)$ & $2(10.53 \%)$ & & $12(63.16 \%)$ & & $5(26.32 \%)$ \\
\hline Zhang, Cao & 289 & $\begin{array}{l}240 \\
(83 \%)\end{array}$ & $10(4.17 \%)$ & $6(2.50 \%)$ & & & & $\begin{array}{l}224 \\
(93.33 \%)\end{array}$ & $\begin{array}{l}49 \\
(16 \%)\end{array}$ & $4(8.16 \%)$ & $8(16.33 \%)$ & & & & $\begin{array}{l}37 \\
(75.51 \%)\end{array}$ \\
\hline Gupta & 496 & $\begin{array}{l}255 \\
(51 \%)\end{array}$ & & & 15 (5.88\%) & & $80(31.37 \%)$ & $\begin{array}{l}160 \\
(62.75 \%)\end{array}$ & $\begin{array}{l}241 \\
(48 \%)\end{array}$ & & & $21(8.71 \%)$ & $77(31.95 \%)$ & & $\begin{array}{l}143 \\
(59.34 \%)\end{array}$ \\
\hline Soares & 1,152 & $\begin{array}{l}696 \\
(60 \%)\end{array}$ & $38(5.46 \%)$ & & & & 658 (94.54\%) & - & $\begin{array}{l}456 \\
(39 \%)\end{array}$ & $39(8.55 \%)$ & & & - & $417(91.45 \%)$ & - \\
\hline Thompson & 470 & $\begin{array}{l}301 \\
(64 \%)\end{array}$ & $39(12.96 \%)$ & $79(26.25 \%)$ & & $\begin{array}{l}183 \\
(60.80 \%)\end{array}$ & & - & $\begin{array}{l}169 \\
(35 \%)\end{array}$ & $27(15.98 \%)$ & $49(28.99 \%)$ & & $93(55.03 \%)$ & & - \\
\hline Bernaola & 1,645 & $\begin{array}{l}1382 \\
(84 \%)\end{array}$ & $35(2.53 \%)$ & $\begin{array}{l}146 \\
(10.56 \%)\end{array}$ & - & $\begin{array}{l}1201 \\
(86.90 \%)\end{array}$ & & - & $\begin{array}{l}263 \\
(15 \%)\end{array}$ & $6(2.28 \%)$ & $33(12.55 \%)$ & & $\begin{array}{l}218 \\
(82.89 \%)\end{array}$ & & - \\
\hline
\end{tabular}




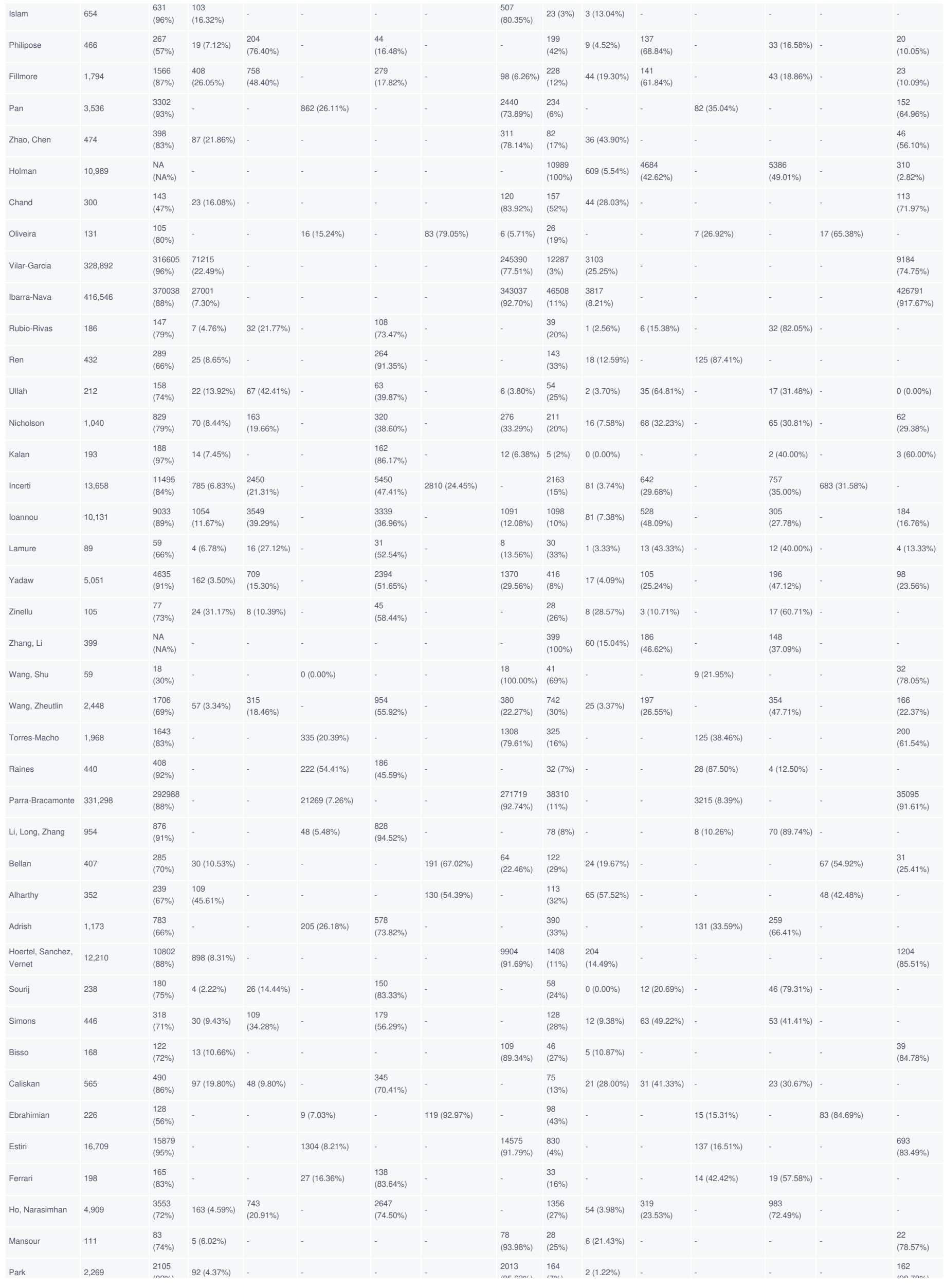




\begin{tabular}{|c|c|c|c|c|c|c|c|c|c|c|c|c|c|c|c|}
\hline & & $(y<\%)$ & & & & & & (50.00\%) & $(1 \%)$ & & & & & & $(50.10 \%)$ \\
\hline Quan & 2,038 & $\begin{array}{l}1587 \\
(77 \%)\end{array}$ & & & $589(37.11 \%)$ & & & $\begin{array}{l}998 \\
(62.89 \%)\end{array}$ & $\begin{array}{l}442 \\
(21 \%)\end{array}$ & & & $218(49.32 \%)$ & & - & $\begin{array}{l}224 \\
(50.68 \%)\end{array}$ \\
\hline Saurabh & 911 & $\begin{array}{l}870 \\
(95 \%)\end{array}$ & $70(8.05 \%)$ & $4(0.46 \%)$ & - & $\begin{array}{l}796 \\
(91.49 \%)\end{array}$ & - & - & $41(4 \%)$ & $4(9.76 \%)$ & $2(4.88 \%)$ & - & $35(85.37 \%)$ & - & - \\
\hline Strangfeld & 3,729 & $\begin{array}{l}3339 \\
(89 \%)\end{array}$ & - & - & $664(19.89 \%)$ & $\begin{array}{l}2190 \\
(65.59 \%)\end{array}$ & - & $\begin{array}{l}485 \\
(14.53 \%)\end{array}$ & $\begin{array}{l}390 \\
(10 \%)\end{array}$ & - & - & $112(28.72 \%)$ & $\begin{array}{l}198 \\
(50.77 \%)\end{array}$ & - & $\begin{array}{l}80 \\
(20.51 \%)\end{array}$ \\
\hline Nezhadmoghadam & 13,367 & $\begin{array}{l}7757 \\
(58 \%)\end{array}$ & $686(8.84 \%)$ & - & & - & - & $\begin{array}{l}7071 \\
(91.16 \%)\end{array}$ & $\begin{array}{l}5610 \\
(41 \%)\end{array}$ & $\begin{array}{l}565 \\
(10.07 \%)\end{array}$ & - & - & - & - & $\begin{array}{l}5045 \\
(89.93 \%)\end{array}$ \\
\hline Nuno & 4,730 & $\begin{array}{l}4401 \\
(93 \%)\end{array}$ & $427(9.70 \%)$ & & - & $\cdot$ & - & $\begin{array}{l}3974 \\
(90.30 \%)\end{array}$ & $\begin{array}{l}329 \\
(6 \%)\end{array}$ & $53(16.11 \%)$ & & - & & - & $\begin{array}{l}276 \\
(83.89 \%)\end{array}$ \\
\hline $\begin{array}{l}\text { Fernandez, } \\
\text { Fuertes }\end{array}$ & 46 & $\begin{array}{l}45 \\
(97 \%)\end{array}$ & $12(26.67 \%)$ & & - & & & $\begin{array}{l}33 \\
(73.33 \%)\end{array}$ & $1(2 \%)$ & $0(0.00 \%)$ & & & & - & $\begin{array}{l}1 \\
(100.00 \%)\end{array}$ \\
\hline Dambha, Miller & 5,328 & $\begin{array}{l}4684 \\
(87 \%)\end{array}$ & $447(9.54 \%)$ & $\begin{array}{l}2326 \\
(49.66 \%)\end{array}$ & - & $\begin{array}{l}1912 \\
(40.82 \%)\end{array}$ & - & - & $\begin{array}{l}664 \\
(12 \%)\end{array}$ & $40(6.02 \%)$ & $\begin{array}{l}388 \\
(58.43 \%)\end{array}$ & & $\begin{array}{l}216 \\
(32.53 \%)\end{array}$ & - & \\
\hline $\begin{array}{l}\text { Williamson, } \\
\text { Tazare }\end{array}$ & 7,999 & $\begin{array}{l}\text { NA } \\
(\mathrm{NA} \%)\end{array}$ & - & & - & - & - & - & $\begin{array}{l}7999 \\
(100 \%)\end{array}$ & $737(9.21 \%)$ & $\begin{array}{l}4745 \\
(59.32 \%)\end{array}$ & - & $\begin{array}{l}2499 \\
(31.24 \%)\end{array}$ & - & $18(0.23 \%)$ \\
\hline Dayem, Ullah & 217 & $\begin{array}{l}164 \\
(75 \%)\end{array}$ & $23(14.02 \%)$ & $72(43.90 \%)$ & - & $\begin{array}{l}67 \\
(40.85 \%)\end{array}$ & - & $2(1.22 \%)$ & $\begin{array}{l}53 \\
(24 \%)\end{array}$ & $2(3.77 \%)$ & $37(69.81 \%)$ & - & $14(26.42 \%)$ & - & - \\
\hline Macias, Guzman & 196 & $\begin{array}{l}109 \\
(55 \%)\end{array}$ & - & - & $37(33.94 \%)$ & - & - & $\begin{array}{l}72 \\
(66.06 \%)\end{array}$ & $\begin{array}{l}87 \\
(44 \%)\end{array}$ & - & - & $34(39.08 \%)$ & - & - & $\begin{array}{l}53 \\
(60.92 \%)\end{array}$ \\
\hline Martin, Vicente & 92 & $\begin{array}{l}38 \\
(41 \%)\end{array}$ & $3(7.89 \%)$ & & - & & & $\begin{array}{l}35 \\
(92.11 \%)\end{array}$ & $\begin{array}{l}54 \\
(58 \%)\end{array}$ & $2(3.70 \%)$ & & & & & $\begin{array}{l}52 \\
(96.30 \%)\end{array}$ \\
\hline Greenbaum & 44 & $\begin{array}{l}32 \\
(72 \%)\end{array}$ & $2(6.25 \%)$ & $6(18.75 \%)$ & - & $\begin{array}{l}24 \\
(75.00 \%)\end{array}$ & - & - & $\begin{array}{l}12 \\
(27 \%)\end{array}$ & $0(0.00 \%)$ & $3(25.00 \%)$ & - & $9(75.00 \%)$ & - & - \\
\hline Asem & 8,162 & $\begin{array}{l}7573 \\
(92 \%)\end{array}$ & $\begin{array}{l}1147 \\
(15.15 \%)\end{array}$ & - & & - & - & $\begin{array}{l}6426 \\
(84.85 \%)\end{array}$ & $\begin{array}{l}589 \\
(7 \%)\end{array}$ & $\begin{array}{l}208 \\
(35.31 \%)\end{array}$ & - & - & . & - & $\begin{array}{l}381 \\
(64.69 \%)\end{array}$ \\
\hline Gil & 186 & $\begin{array}{l}174 \\
(93 \%)\end{array}$ & $42(24.14 \%)$ & - & & - & $132(75.86 \%)$ & & $12(6 \%)$ & $4(33.33 \%)$ & - & - & - & $8(66.67 \%)$ & - \\
\hline Rubina & 52 & $\begin{array}{l}41 \\
(78 \%)\end{array}$ & $2(4.88 \%)$ & & - & & $35(85.37 \%)$ & $4(9.76 \%)$ & $\begin{array}{l}11 \\
(21 \%)\end{array}$ & $0(0.00 \%)$ & & - & & $6(54.55 \%)$ & $5(45.45 \%)$ \\
\hline Mann & 1,769 & $\begin{array}{l}1422 \\
(80 \%)\end{array}$ & & & $515(36.22 \%)$ & $\begin{array}{l}840 \\
(59.07 \%)\end{array}$ & & $67(4.71 \%)$ & $\begin{array}{l}347 \\
(19 \%)\end{array}$ & & & $130(37.46 \%)$ & $\begin{array}{l}163 \\
(46.97 \%)\end{array}$ & - & $\begin{array}{l}54 \\
(15.56 \%)\end{array}$ \\
\hline Madakkatel & 1,953 & $\begin{array}{l}1577 \\
(80 \%)\end{array}$ & $\begin{array}{l}184 \\
(11.67 \%)\end{array}$ & $\begin{array}{l}586 \\
(37.16 \%)\end{array}$ & - & $\begin{array}{l}789 \\
(50.03 \%)\end{array}$ & - & $18(1.14 \%)$ & $\begin{array}{l}376 \\
(19 \%)\end{array}$ & $54(14.36 \%)$ & $\begin{array}{l}176 \\
(46.81 \%)\end{array}$ & - & $\begin{array}{l}140 \\
(37.23 \%)\end{array}$ & - & $6(1.60 \%)$ \\
\hline Demichev & 50 & $\begin{array}{l}35 \\
(70 \%)\end{array}$ & $1(2.86 \%)$ & $6(17.14 \%)$ & - & $\begin{array}{l}28 \\
(80.00 \%)\end{array}$ & - & - & $\begin{array}{l}15 \\
(30 \%)\end{array}$ & $0(0.00 \%)$ & $2(13.33 \%)$ & - & $13(86.67 \%)$ & - & - \\
\hline $\begin{array}{l}\text { Velasco, } \\
\text { Rodriguez }\end{array}$ & 2,070 & $\begin{array}{l}1677 \\
(81 \%)\end{array}$ & $68(4.05 \%)$ & & - & - & & $\begin{array}{l}1599 \\
(95.35 \%)\end{array}$ & $\begin{array}{l}393 \\
(18 \%)\end{array}$ & $17(4.33 \%)$ & & - & & - & $\begin{array}{l}376 \\
(95.67 \%)\end{array}$ \\
\hline Puebla, Neira & 10,216 & $\begin{array}{l}10028 \\
(98 \%)\end{array}$ & $395(3.94 \%)$ & $870(8.68 \%)$ & - & $\begin{array}{l}8763 \\
(87.39 \%)\end{array}$ & - & - & $\begin{array}{l}188 \\
(1 \%)\end{array}$ & $3(1.60 \%)$ & $47(25.00 \%)$ & - & $\begin{array}{l}138 \\
(73.40 \%)\end{array}$ & - & - \\
\hline Prats-Uribe, Xie & 1,591 & $\begin{array}{l}1219 \\
(76 \%)\end{array}$ & $\begin{array}{l}136 \\
(11.16 \%)\end{array}$ & $\begin{array}{l}453 \\
(37.16 \%)\end{array}$ & & $\begin{array}{l}630 \\
(51.68 \%)\end{array}$ & & & $\begin{array}{l}372 \\
(23 \%)\end{array}$ & $56(15.05 \%)$ & $\begin{array}{l}175 \\
(47.04 \%)\end{array}$ & & $\begin{array}{l}141 \\
(37.90 \%)\end{array}$ & & \\
\hline Peng, Lei & 622 & $\begin{array}{l}547 \\
(87 \%)\end{array}$ & $48(8.78 \%)$ & & - & - & - & $\begin{array}{l}499 \\
(91.22 \%)\end{array}$ & $\begin{array}{l}75 \\
(12 \%)\end{array}$ & $14(18.67 \%)$ & & - & & - & $\begin{array}{l}61 \\
(81.33 \%)\end{array}$ \\
\hline Nassar & 160 & $\begin{array}{l}121 \\
(75 \%)\end{array}$ & $9(7.44 \%)$ & - & & - & - & $\begin{array}{l}112 \\
(92.56 \%)\end{array}$ & $\begin{array}{l}39 \\
(24 \%)\end{array}$ & $8(20.51 \%)$ & - & - & - & - & $\begin{array}{l}31 \\
(79.49 \%)\end{array}$ \\
\hline $\begin{array}{l}\text { Mendez, } \\
\text { Dominguez }\end{array}$ & 7,064 & $\begin{array}{l}6867 \\
(97 \%)\end{array}$ & $132(1.92 \%)$ & - & & - & - & $\begin{array}{l}6735 \\
(98.08 \%)\end{array}$ & $\begin{array}{l}197 \\
(2 \%)\end{array}$ & $2(1.02 \%)$ & - & - & - & - & $\begin{array}{l}195 \\
(98.98 \%)\end{array}$ \\
\hline Marimuthu & 854 & $\begin{array}{l}767 \\
(89 \%)\end{array}$ & $4(0.52 \%)$ & & - & & & $\begin{array}{l}763 \\
(99.48 \%)\end{array}$ & $\begin{array}{l}87 \\
(10 \%)\end{array}$ & $6(6.90 \%)$ & & - & & - & $\begin{array}{l}81 \\
(93.10 \%)\end{array}$ \\
\hline Chetboun & 3,401 & $\begin{array}{l}2892 \\
(85 \%)\end{array}$ & 190 (6.57\%) & $\begin{array}{l}689 \\
(23.82 \%)\end{array}$ & - & $\begin{array}{l}1756 \\
(60.72 \%)\end{array}$ & - & $\begin{array}{l}257 \\
(8.89 \%)\end{array}$ & $\begin{array}{l}509 \\
(14 \%)\end{array}$ & $20(3.93 \%)$ & $\begin{array}{l}171 \\
(33.60 \%)\end{array}$ & - & $\begin{array}{l}164 \\
(32.22 \%)\end{array}$ & - & $\begin{array}{l}154 \\
(30.26 \%)\end{array}$ \\
\hline Bruce & 125 & $\begin{array}{l}101 \\
(80 \%)\end{array}$ & $13(12.87 \%)$ & - & - & - & - & $\begin{array}{l}88 \\
(87.13 \%)\end{array}$ & $\begin{array}{l}24 \\
(19 \%)\end{array}$ & $12(50.00 \%)$ & - & - & - & - & $\begin{array}{l}12 \\
(50.00 \%)\end{array}$ \\
\hline Bhaskaran & 1,584 & $\begin{array}{l}1082 \\
(68 \%)\end{array}$ & $85(7.86 \%)$ & $\begin{array}{l}381 \\
(35.21 \%)\end{array}$ & - & $\begin{array}{l}587 \\
(54.25 \%)\end{array}$ & - & $29(2.68 \%)$ & $\begin{array}{l}502 \\
(31 \%)\end{array}$ & $27(5.38 \%)$ & $\begin{array}{l}224 \\
(44.62 \%)\end{array}$ & - & $\begin{array}{l}237 \\
(47.21 \%)\end{array}$ & - & $14(2.79 \%)$ \\
\hline Bertuzzi & $17,456,515$ & $\begin{array}{l}\text { NA } \\
(\mathrm{NA} \%)\end{array}$ & & & - & - & - & - & $\begin{array}{l}17063 \\
(0 \%)\end{array}$ & $\begin{array}{l}1109 \\
(6.50 \%)\end{array}$ & $\begin{array}{l}10276 \\
(60.22 \%)\end{array}$ & - & $\begin{array}{l}5678 \\
(33.28 \%)\end{array}$ & - & \\
\hline Avouac & 92 & $\begin{array}{l}70 \\
(76 \%)\end{array}$ & $10(14.29 \%)$ & & & & 35 (50.00\%) & $\begin{array}{l}25 \\
(35.71 \%)\end{array}$ & $\begin{array}{l}22 \\
(23 \%)\end{array}$ & $6(27.27 \%)$ & & & & $5(22.73 \%)$ & $\begin{array}{l}11 \\
(50.00 \%)\end{array}$ \\
\hline Alsafar & 873 & $\begin{array}{l}751 \\
(86 \%)\end{array}$ & $\begin{array}{l}101 \\
(13.45 \%)\end{array}$ & & - & & & $\begin{array}{l}650 \\
(86.55 \%)\end{array}$ & $\begin{array}{l}122 \\
(13 \%)\end{array}$ & $36(29.51 \%)$ & & & & & $\begin{array}{l}86 \\
(70.49 \%)\end{array}$ \\
\hline Ahmadi & 464 & $\begin{array}{l}438 \\
(94 \%)\end{array}$ & $50(11.42 \%)$ & & - & - & $388(88.58 \%)$ & - & $26(5 \%)$ & $0(0.00 \%)$ & & - & & $26(100.00 \%)$ & - \\
\hline Aboueshia & 468,443 & $\begin{array}{l}468056 \\
(99 \%)\end{array}$ & $\begin{array}{l}48522 \\
(10.37 \%)\end{array}$ & $\begin{array}{l}162807 \\
(34.78 \%)\end{array}$ & & $\begin{array}{l}256727 \\
(54.85 \%)\end{array}$ & - & - & $\begin{array}{l}387 \\
(0 \%)\end{array}$ & $59(15.25 \%)$ & $\begin{array}{l}183 \\
(47.29 \%)\end{array}$ & - & $\begin{array}{l}145 \\
(37.47 \%)\end{array}$ & - & - \\
\hline
\end{tabular}


Forest plot of current smokers and mortality

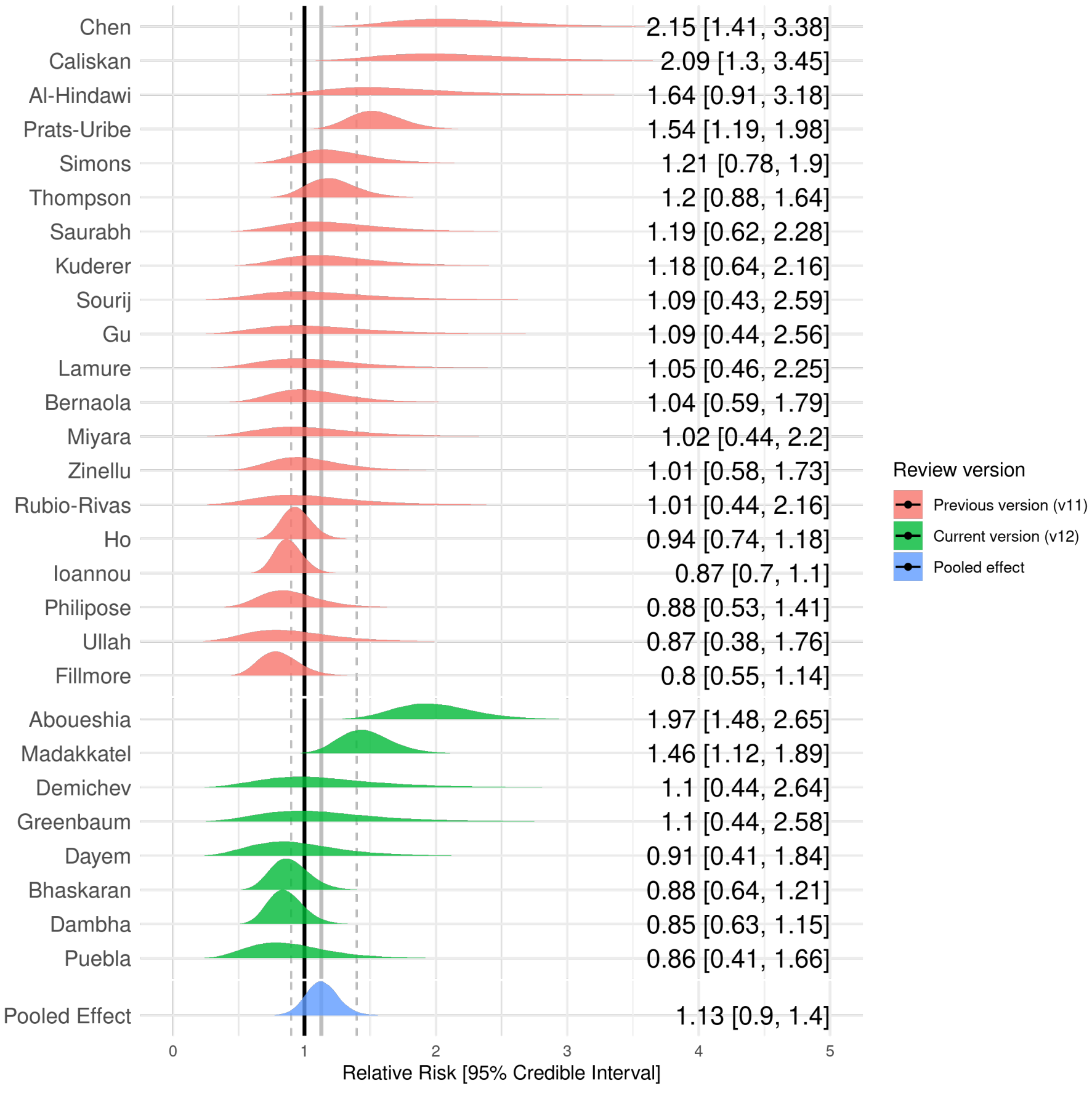


Forest plot of former smokers and mortality

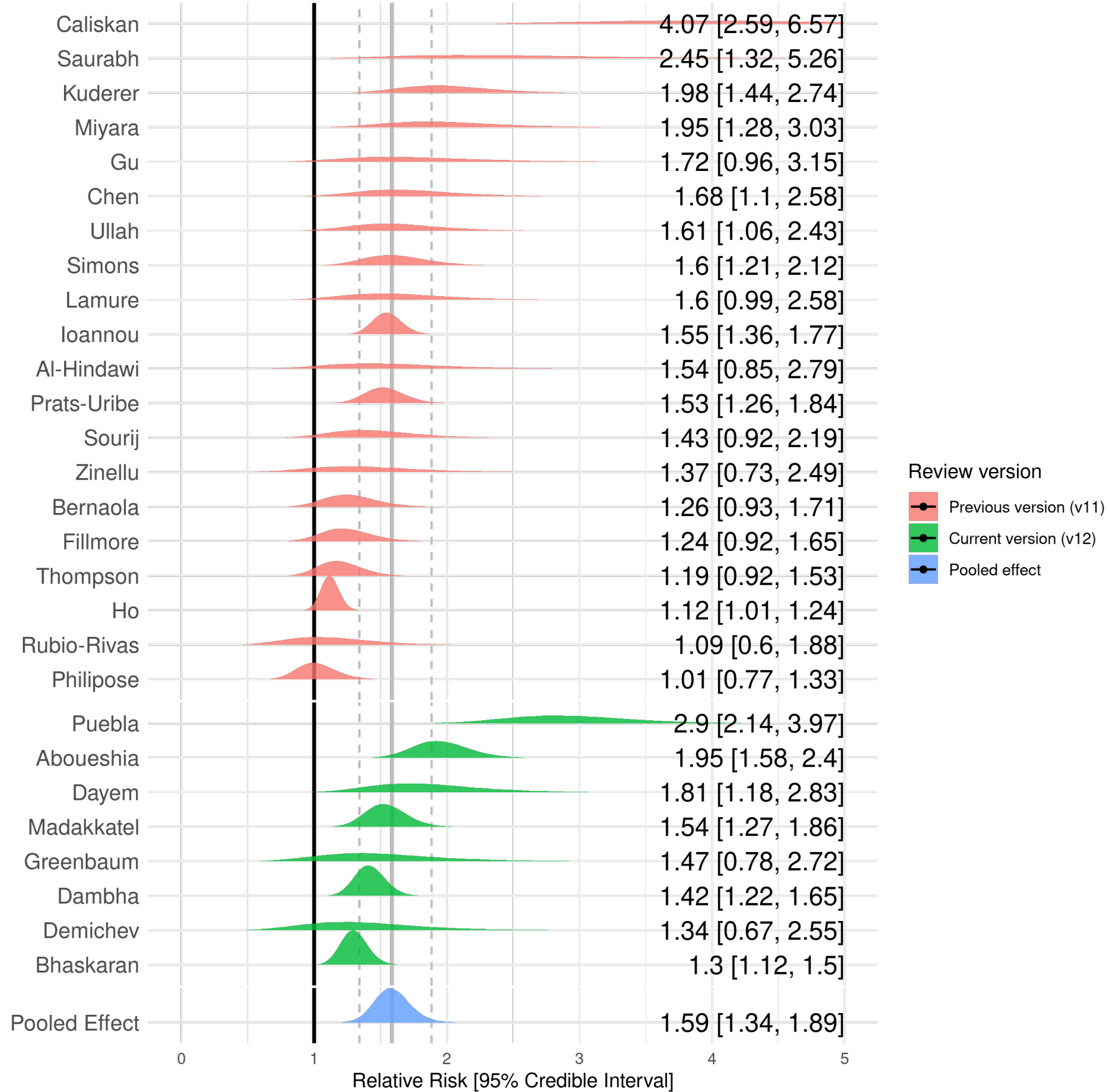

v11 mortality prior

This living rapid review found uncertainty in the majority of 547 studies arising from the recording of smoking status. Notwithstanding this uncertainty, compared with overall adult national prevalence estimates, recorded current smoking rates in most studies were lower than expected. In a subset of good and fair quality studies ( $n=39$ ), current but not former smokers had a reduced risk of testing positive for SARS-CoV-2 but current (and former) smokers appeared somewhat more likely to present for testing and/or receive a test. Current compared with never smokers had an increased risk of greater disease severity in hospitalised patients. Data for current smokers on the risk of hospitalisation and mortality were inconclusive and favoured there being no important associations. Former smokers were at increased risk of hospitalisation, disease severity and mortality compared with never smokers. 
1. Exposure to the SARS-CoV-2 virus is heterogeneous with different subgroups at heightened risk of infection at different stages of the pandemic, at least partly due to differential contact matrices by age, sex and socioeconomic position (CMMID COVID-19 working group et al., 2020), which are associated with smoking status.

2. The probability of viral exposure depends largely on local prevalence, which varies over time. This likely introduces bias in studies assessing the rate of infection by smoking status conducted in the early phase of the pandemic.

2. Infection with SARS-CoV-2

1. Infection following viral exposure depends on individual differences in, for example, genetic susceptibility or immunocompetence, which are poorly understood at present and may be confounded with smoking. For example, the household secondary attack rate for COVID-19 is estimated at 17\% (Fung et al., 2020).

2. Heated and humidified air may act to disrupt the ability of the virus to persist in the airway mucosa of smokers. There is some evidence that transient localised hyperthermia can inhibit replication of rhinoviruses, a non-enveloped virus that causes the common cold (Conti et al., 1999). However, as SARS-CoV-2 is an enveloped virus (Schoeman \& Fielding, 2019), it is unclear whether a similar protective effect against viral replication or invasion by heated and humidified air may occur.

3. Symptomatic COVID-19

1. An estimated $20 \%(95 \% \mathrm{Cl}=17-25 \%)$ of COVID-19 cases are asymptomatic (Buitrago-Garcia et al., 2020), with some evidence suggesting younger people are more likely to be asymptomatic (Kronbichler et al., 2020). Testing is hence likely limited in some subgroups, with the potential for these groups to include an overrepresentation of current smokers. The proportion of symptomatic cases may also vary depending on viral strain and vaccination status.

2. On the other hand, current and former smokers may be more likely to meet local criteria for community testing due to increased prevalence of symptoms consistent with SARS-CoV-2 infection, such as cough, increased sputum production or altered sense of smell or taste (Hopkinson et al., 2020). Evidence from a small number of studies indicates that current smokers may be more likely to present for testing, hence increasing the denominator in comparisons with never smokers and potentially inflating the rate of negative tests in current smokers. Infection positivity rates estimated among random samples are more informative. We identified nine random/population studies reporting on seroprevalence and smoking status. However, response rates were suboptimal where reported (e.g. 58.8\%-64.3\%; (Merkely et al., 2020) and (Wagner et al., 2021)) and the current smoking rate was often several percentage points below national prevalence estimates (e.g. $12 \%$ vs. $25 \%$ and $15 \%$ vs. $27 \%$ daily smoking prevalence, respectively; (Andler, 2019) and (statistique, 2020)), which raises doubt about the representativeness of the final samples.

4. Testing positive for SARS-CoV-2

1. Smokers with COVID-19 may be less likely to receive a SARS-CoV-2 test or present to hospital due to lack of access to healthcare and may be more likely to die in the community from sudden complications (i.e. self-selection bias) and thus not be recorded (Brown, 2020).

2. Diagnostic criteria for SARS-CoV-2 infection and COVID-19 have changed during the course of the pandemic (WHO, 2020). It was not possible to extract details on the specific RT-PCR or antibody-based techniques or platforms used across the included studies due to reporting gaps. Different platforms have varying sensitivity and specificity to detect SARS-CoV-2 infection. In addition, testing for acute infection requires swabbing of the mucosal epithelium, which may be disrupted in current smokers, potentially altering the sensitivity of assays (Lusignan et al., 2020).

5. Hospitalisation with COVID-19

1. Reasons for hospitalisation vary by country and time in the pandemic. For example, early cases may have been hospitalised for isolation and quarantine reasons and not due to medical necessity. It is plausible this may have skewed early data towards less severe cases. In addition, the observed association between former smoking and greater disease severity may be explained by collider bias (Griffith et al., 2020), where conditioning on a collider (e.g. testing or hospitalisation) by design or analysis may introduce a spurious association between current or former smoking (a potential cause of testing or hospitalisation) and SARS-CoV-2 infection/adverse outcomes from COVID-19 (potentially exacerbated by smoking) (Murray, 2020).

2. The majority of included studies relied on EHRs as the source of information on smoking status. Research shows large discrepancies between EHRs and actual behaviour (Polubriaginof et al., 2018). Known failings of EHRs include implausible longitudinal changes, such as former smokers being recorded as never smokers at subsequent hospital visits (Polubriaginof et al., 2018). A recent study conducted in a UK hospital setting found a large discrepancy between smoking status recorded on the EHR and within the contemporaneous clinical notes (Simons, Perski, et al., 2020). Known failings of EHRs include implausible longitudinal changes, such as former smokers being recorded as never smokers at subsequent hospital visits (Polubriaginof et al., 2018). Misreporting on the part of the patient (perhaps due to perceived stigma) has also been observed, with biochemical measures showing higher rates of smoking compared with self-report in hospitalised patients in the US (Benowitz et al., 2009). It is hence likely that under-reporting of current and former smoking status in hospitals occurred across the included studies.

3. The majority of included studies were conducted in hospital settings. It is plausible that a non-trivial proportion of patients were infected with SARS-CoV-2 while being an inpatient for a different medical reason. If so, this may have biased the hospitalised populations towards older and more frail groups, who are less likely to be smokers (Mangera et al., 2017).

4. Individuals with severe COVID-19 symptoms may have stopped smoking immediately before admission to hospital and may therefore not have been recorded as current smokers (i.e. reverse causality).

6. COVID-19 disease severity and death

1. Given lack of knowledge of the disease progression and long-term outcomes of COVID-19, it is unclear whether studies conducted thus far in the pandemic have monitored patients for a sufficient time period to report complete survival outcomes or whether they are subject to early censoring. Adding to this, COVID-19 related mortality has been differentially defined across countries and epidemic phases. For example, in some UK reporting, death within 28 days of a COVID-19 diagnosis is required for attributing the cause of death to the virus. However, according to the UK Office for National Statistics, COVID-19 deaths are recorded only if this was stated on the death certificate.

2. If there is a protective effect of nicotine on COVID-19 disease outcomes, abrupt nicotine withdrawal upon hospitalisation may lead to worse disease outcomes including death (Farsalinos, Niaura, et al., 2020).

3. During periods of heightened demand of limited healthcare resources, current and former smokers with extensive comorbidities may have reduced priority for intensive care admission, thus leading to higher in-hospital mortality.

4. COVID-19 outcomes are currently limited to in-hospital death or survival to discharge. This binary outcome does not capture potential long-term morbidity attributed to COVID-19, such as stroke, amputation, acute cardiac events, or long COVID (i.e. symptoms that persist for weeks or months beyond infection), which may be moderated by smoking status. 
Infection following exposure depends on poorly understood individual differences (e.g. genetic susceptibility, immunocompetence)

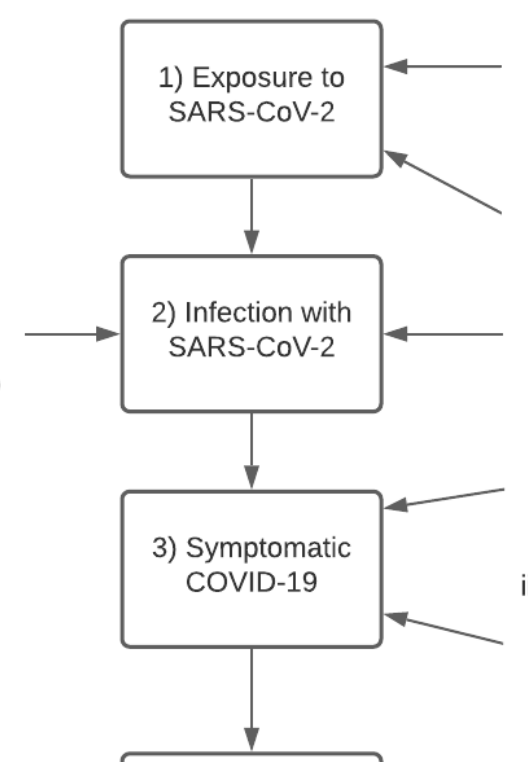

i. Differential access to health care (inequities limiting access to testing or healthcare services)

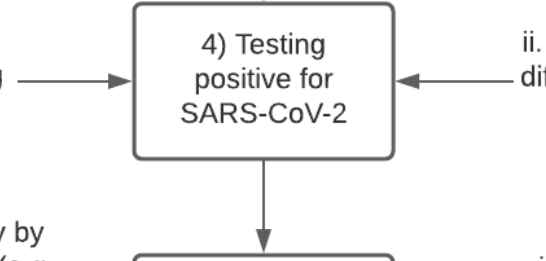

i. Reasons for hospitalisation vary by country and time in the pandemic (e.g. hospitalisation for isolation and quarantine reasons)

iii. Hospital acquired infections

i. It is unclear whether studies have monitored patients for a sufficient period of time to report complete survival outcomes. Mortality from COVID-19 has also been differentially defined across countries and studies i. Exposure to the virus is heterogeneous due to

differential contact matrices by age, sex and socioeconomic position, which are associated with smoking

ii. Probability of exposure to infected individuals depends on local prevalence, which is time-varying

ii. Heated and humidified air may act to disrupt the ability of the virus to persist in the airway mucosa of smokers

i. Asymptomatic infection, particularly among younger individuals, may limit testing. Age is associated with smoking status

ii. Symptoms consistent with COVID-19 such as cough or change in smell or taste may be more common in current or former smokers, thus increasing numbers presenting for testing

ii. Sensitivity of tests may differ in current smokers and different techniques and platforms used throughout the pandemic have varying sensitivity and specificity

ii. Misreporting or poor recording of smoking status

iv. Reverse causality (e.g. current smokers quitting immediately prior to hospitalisation)

ii. Acute nicotine withdrawal may lead to increased disease severity in hospitalised current smokers

iii. Current and former smokers may have increased comorbidities compared with never smokers. Intensive care escalation (as judged by healthcare professionals in discussion with patients) may not be in their best interest.

iv. COVID-19 outcomes are currently limited to death or recovery, with little attention given to long-term morbidity 
mitigate some of the limitations of healthcare based observational studies. The comparisons of current and former smoking prevalence in the included studies with national prevalence estimates did not adjust observed prevalence for the demographic profile of those tested/admitted to hospital. Other reviews focused on this comparison have applied adjustments for sex and age, and continue to find lower than expected prevalence - notwithstanding the issues complicating interpretation described above (Farsalinos, Barbouni, et al., 2020).

Implications for research, policy and practice

Further scientific research is needed to resolve the mixed findings summarised in our review. First, clinical trials of the posited therapeutic effect of nicotine could have important implications both for smokers and for improved understanding of how the SARS-CoV-2 virus causes disease in humans. Such trials should focus on medicinal nicotine (as smoked tobacco is a dirty delivery mechanism that could mask beneficial effects) and potentially differentiate between different modes of delivery (i.e. inhaled vs. ingested) since this can affect pharmacokinetics (Shahab et al., 2013) and potential therapeutic effects. Of note, a recent study of rhesus macaques reported that the nAChR agonist varenicline has the potential to interact with and inhibit SARS-CoV-2 infection and replication. A second research priority would be a large, representative (randomly sampled) population survey with a validated assessment of smoking status which distinguishes between recent and long-term exsmokers - ideally biochemically verified - and assesses seroprevalence and links to health records.

In the meantime, public-facing messages about the possible protective effect of smoking or nicotine are premature. In our view, until there is further research, the quality of the evidence does not justify the huge risk associated with a message likely to reach millions of people that a lethal activity, such as smoking, may protect against COVID-19. It continues to be appropriate to recommend smoking cessation and emphasise the role of alternative nicotine products to support smokers to stop as part of public health efforts during COVID-19. At the very least, smoking cessation reduces acute risks from cardiovascular disease and could reduce demands on the healthcare system (Stead et al., 2013). GPs and other healthcare providers can play a crucial role - brief, high-quality and free online training is available at National Centre for Smoking Cessation and Training.

\section{Conclusion}

Across 547 studies, recorded current but not past smoking prevalence was generally lower than national prevalence estimates. Current smokers were at reduced risk of testing positive for SARSCoV-2 and at increased risk of greater in-hospital disease severity. Former smokers were at increased risk of hospitalisation, in-hospital disease severity and mortality compared with never smokers.

\section{Acknowledgements}

An original short review for the Royal College of Physicians was converted to an extended living review after a request by Martin Dockrell, Tobacco Control Lead, Public Health England. All scientific decisions were made by the authors independently of funders and external organisations. The authors would like to thank Rosemary Koper for her assistance in running the electronic searches and data extraction up until v7, and all authors who responded to requests for additional data.

Declaration of conflicts of interest

DS and OP have no conflicts of interest to declare. LS has received a research grant and honoraria for a talk and travel expenses from manufacturers of smoking cessation medications (Pfizer and Johnson \& Johnson). JB has received unrestricted research funding to study smoking cessation from companies who manufacture smoking cessation medications. All authors declare no financial links with tobacco companies or e-cigarette manufacturers or their representatives.

\section{Funding statement}

DS is supported by a PhD studentship from the UK Biotechnology and Biological Sciences Research Council [BB/M009513/1]. OP receives salary support from Cancer Research UK (C1417/A22962). JB, LS, \& OP are members of SPECTRUM, a UK Prevention Research Partnership Consortium (MR/S037519/1). UKPRP is an initiative funded by the UK Research and Innovation Councils, the Department of Health and Social Care (England) and the UK devolved administrations, and leading health research charities.

\section{Review versions}

The most up-to-date review version is available here

Previous review versions

- Version 1

- Version 2

- $\underline{\text { Version } 3}$

- Version 4

- Version 5

- $\underline{\text { Version } 6}$

- Version 7

- Version 8

- Version 9

- Version 10

- Version 11 
Data availability

All data contributing to the current and future review versions are availablehere

All code required to reproduce the current and future analyses are availablehere

\section{References}

Aabakke, A. J., Krebs, L., Petersen, T. G., Kjeldsen, F. S., Corn, G., Wøjdemann, K., Ibsen, M. H., Jonsdottir, F., Rønneberg, E., Andersen, C. S., Sundtoft, I., Clausen, T., Milbak, J., Burmester, L., Lindved, B., Thorsen-Meyer, A., Khalil, M. R., Henriksen, B., Jønsson, L., ... Bliddal, M. (2021). SARS-CoV-2 infection in pregnancy in Denmark - characteristics and outcomes after confirmed infection in pregnancy: A nationwide, prospective, population-based cohort study [Preprint]. Obstetrics; Gynecology. https://doi.org/10.1101/2021.06.08.21258480

Abadom, T. R., Smith, A. D., Tempia, S., Madhi, S. A., Cohen, C., \& Cohen, A. L. (2016). Risk factors associated with hospitalisation for influenza-associated severe acute respiratory illness in South Africa: A case-population study. Vaccine, 34(46), 5649-5655. https://doi.org/10.1016/j.vaccine.2016.09.011

Abajo, F. J. de, Rodríguez-Miguel, A., Rodríguez-Martín, S., Lerma, V., \& García-Lledó, A. (2021).IN-HOSPITAL CONTINUATION WITH ANGIOTENSIN RECEPTOR BLOCKERS IS ASSOCIATED WITH A LOWER MORTALITY RATE THAN CONTINUATION WITH ANGIOTENSIN CONVERTING ENZYME INHIBITORS IN COVID-19 PATIENTS: A RETROSPECTIVE COHORT STUDY. https://doi.org/10.1101/2021.02.01.21250853

Abdulamir, A. S., Gorial, F. I., Saadi, S. J., Maulood, M. F., Hashim, H. A., \& abdulrrazaq, M. K. (2021).Effectiveness and Safety of Niclosamaide as Add-on Therapy to the Standard of Care Measures in COVID-19 Management: Randomized controlled clinical trial [Preprint]. Infectious Diseases (except HIV/AIDS).https://doi.org/10.1101/2021.06.10.21258709

Abolghasemi, S., Mardani, M., Sali, S., Honarvar, N., \& Baziboroun, M. (n.d.). COVID-19 and Kidney Transplant Recipients.Transplant Infectious Disease, n/a(n/a), e13413. https://doi.org/10.1111/tid.13413

Aboueshia, M., Hussein, M. H., Attia, A. S., Swinford, A., Miller, P., Omar, M., Toraih, E. A., Saba, N., Safah, H., Duchesne, J., \& Kandil, E. (2021). Cancer and COVID-19: Analysis of patient outcomes. Future Oncology, fon-2021-0121. https://doi.org/10.2217/fon-2021-0121

Action to beat coronavirus/Action pour battre le coronavirus (Ab-C) Study Investigators, \& Jha, P. (2021).COVID Seroprevalence, Symptoms and Mortality During the First Wave of SARS-CoV-2 in Canada [Preprint]. Public; Global Health. https://doi.org/10.1101/2021.03.04.21252540

Ader, F., Peiffer-Smadja, N., Poissy, J., Bouscambert-Duchamp, M., Belhadi, D., Diallo, A., Delmas, C., Saillard, J., Dechanet, A., Mercier, N., Dupont, A., Alfaiate, T., Lescure, F.-X., Raffi, F., Goehringer, F., Kimmoun, A., Jaureguiberry, S., Reignier, J., Nseir, S., ... Mentre, F. (2021). Antiviral drugs in hospitalized patients with COVID-19 - the DisCoVeRy trial https://doi.org/10.1101/2021.01.08.20248149

Adrish, M., Chilimuri, S., Mantri, N., Sun, H., Zahid, M., Gongati, S., Fortuzi, K., Jog, A. P., Purmessur, P., \& Singhal, R. (2020). Association of smoking status with outcomes in hospitalised patients with COVID-19. BMJ Open Respiratory Research, 7(1), e000716. https://doi.org/10.1136/bmiresp-2020-000716

Ahmadi, M. N., Huang, B.-H., Inan-Eroglu, E., Hamer, M., \& Stamatakis, E. (2021). Lifestyle risk factors and infectious disease mortality, including COVID-19, among middle aged and older adults: Evidence from a community-based cohort study in the United Kingdom. Brain, Behavior, and Immunity, 96, 18-27. https://doi.org/10.1016/.bbi.2021.04.022

Aksu, K., Naziroğlu, T., \& Özkan, P. (2020). Factors determining COVID-19 pneumonia severity in a country with routine BCG vaccination.Clinical \& Experimental Immunology, 202(2), $220-225$. https://doi.org/10.1111/cei.13507

Alguwaihes, A. M., Al-Sofiani, M. E., Megdad, M., Albader, S. S., Alsari, M. H., Alelayan, A., Alzahrani, S. H., Sabico, S., Al-Daghri, N. M., \& Jammah, A. A. (2020). Diabetes and Covid-19 among hospitalized patients in Saudi Arabia: A single-centre retrospective study. Cardiovascular Diabetology, 19(1), 205. https://doi.org/10.1186/s12933-020-01184-4

Alhamlan, F. S., Almaghrabi, R. S., Devol, E. B., Alotaibi, A. B., Alageel, S. M., Obeid, D. A., Alraddadi, B. M., Althawadi, S. I., Mutabagani, M. S., \& Al-Qahtani, A. A. (2021)Epidemiology and Clinical Characteristics in Individuals with Confirmed SARS-CoV-2 Infection During the Early COVID-19 Pandemic in Saudi Arabia [Preprint]. Epidemiology. https://doi.org/10.1101/2021.07.13.21260428

Alharthy, A., Aletreby, W., Faqihi, F., Balhamar, A., Alaklobi, F., Alanezi, K., Jaganathan, P., Tamim, H., Alqahtani, S. A., Karakitsos, D., \& Memish, Z. A. (2020). Clinical Characteristics and Predictors of 28-Day Mortality in 352 Critically III Patients with COVID-19: A Retrospective Study: Journal of Epidemiology and Global Health https://doi.org/10.2991/jegh.k.200928.001

Al-Hindawi, A., Sokhi, J., Cuddihy, J., Lockie, C., Christie, L., Davies, R., Singh, S., Vizcaychipi, M., Hayes, M., Sisson, A., \& Keays, R. (2020). COVID-19 in London, a Case Series Demonstrating Late Improvement in Survivors. medRxiv, 2020.05.16.20103853. https://doi.org/10.1101/2020.05.16.20103853

Ali, M. R., Hasan, Md. A., Rahman, Md. S., Billah, M., Karmakar, S., Shimu, A. S., Hossain, Md. F., Maruf, Md. M. H., Rahman, Md. S., Saju, Md. S. R., Hasan, M. R., Acharjee, U. K., \& Hasan, Md. F. (2021). Clinical manifestations and socio-demographic status of COVID-19 patients during the second-wave of pandemic: A Bangladeshi experience. Journal of Infection and Public Health S1876034121001775. https://doi.org/10.1016/j.jiph.2021.06.011

Alizadehsani, R., Sani, Z. A., Behjati, M., Roshanzamir, Z., Hussain, S., Abedini, N., Hasanzadeh, F., Khosravi, A., Shoeibi, A., Roshanzamir, M., Moradnejad, P., Nahavandi, S., Khozeimeh, F., Zare, A., Panahiazar, M., Acharya, U. R., \& Islam, S. M. S. (2020). Risk Factors Prediction, Clinical Outcomes, and Mortality of COVID-19 Patients. medRxiv, 2020.07.07.20148569. https://doi.org/10.1101/2020.07.07.20148569

Alkurt, G., Murt, A., Aydin, Z., Tatli, O., Agaoglu, N. B., Irvem, A., Aydin, M., Karaali, R., Gunes, M., Yesilyurt, B., Turkez, H., Mardinoglu, A., Doganay, M., Basinoglu, F., Seyahi, N., Doganay, G. D., \& Doganay, L. (2020). Seroprevalence of Coronavirus Disease 2019 (COVID-19) Among Health Care Workers from Three Pandemic Hospitals of Turkey. medRxiv, 2020.08.19.20178095. 
https://doi.org/10.1101/2020.08.19.20178095

Allenbach, Y., Saadoun, D., Maalouf, G., Vieira, M., Hellio, A., Boddaert, J., Gros, H., Salem, J. E., Resche Rigon, M., Menyssa, C., Biard, L., Benveniste, O., Cacoub, P., \& on behalf of DIMICOVID. (2020). Development of a multivariate prediction model of intensive care unit transfer or death: A French prospective cohort study of hospitalized COVID-19 patients. PLOS ONE, 15(10), e0240711. https://doi.org/10.1371/journal.pone.0240711

Almazeedi, S., Al-Youha, S., Jamal, M. H., Al-Haddad, M., Al-Muhaini, A., Al-Ghimlas, F., \& Al-Sabah, S. (2020). Characteristics, risk factors and outcomes among the first consecutive 1096 patients diagnosed with COVID-19 in Kuwait. EClinicalMedicine, 24, 100448. https://doi.org/10.1016/j.eclinm.2020.100448

Almirall, J., González, C. A., Balanzó, X., \& Bolíbar, I. (1999). Proportion of community-acquired pneumonia cases attributable to tobacco smoking.Chest, 116, 375-379. https://doi.org/10.1378/chest.116.2.375

Alqahtani, J. S., Oyelade, T., Aldhahir, A. M., Alghamdi, S. M., Almehmadi, M., Alqahtani, A. S., Quaderi, S., Mandal, S., \& Hurst, J. (2020). Prevalence, Severity and Mortality associated with COPD and Smoking in patients with COVID-19: A Rapid Systematic Review and Meta-Analysis. medRxiv, 2020.03.25.20043745. https://doi.org/10.1101/2020.03.25.20043745

AISafar, H., Grant, W. B., Hijazi, R., Uddin, M., Alkaabi, N., Tay, G., Mahboub, B., \& Al Anouti, F. (2021). COVID-19 Disease Severity and Death in Relation to Vitamin D Status among SARS-CoV-2Positive UAE Residents. Nutrients, 13(5), 1714. https://doi.org/10.3390/nu13051714

Alshami, A. A., Alattas, R. A., Anan, H. F., Qahtani, H. S. A., Mulhim, M. A. A., Alahilmi, A. A., \& Alfaraj, A. H. (2020). Silent Disease and Loss of Taste and Smell are Common Manifestations of SARS-COV-2 Infection in a Quarantine Facility: First report from Saudi Arabia. medRxiv, 2020.05.13.20100222. https://doi.org/10.1101/2020.05.13.20100222

Altamimi, H., Alahmad, Y., Khazal, F., Elhassan, M., AlBinali, H., Arabi, A., AlQahtani, A., Asaad, N., Al-Hiji, M., Hamid, T., Rafie, I., Omrani, A. S., AlKaabi, S., Alkhal, A., AlMalsImani, M., Ali, M., Alkhani, M., AINesf, M., Jalala, S. A., ... Suwaidi, J. A. (2020). The Outcome of COVID-19 Patients with Acute Myocardial Infarction. medRxiv, 2020.07.21.20156349. https://doi.org/10.1101/2020.07.21.20156349

Altibi, A. M., Bhargava, P., Liaqat, H., Slota, A. A., Sheth, R., Jebbawi, L. A., George, M. E., LeDuc, A., Abdallah, E., Russell, L. R., Jain, S., Shirvanian, N., Masri, A., \& Kak, V. (2020). Comparative Clinical Outcomes and Mortality in Prisoner and Non-Prisoner Populations Hospitalized with COVID-19: A Cohort from Michigan. medRxiv, 2020.08.08.20170787. https://doi.org/10.1101/2020.08.08.20170787

Amanat, M., Rezaei, N., Roozbeh, M., Shojaei, M., Tafakhori, A., Zoghi, A., Darazam, I. A., Salehi, M., Karimialavijeh, E., Lima, B. S., Garakani, A., Vaccaro, A., \& Ramezani, M. (2021). Neurological manifestations as the predictors of severity and mortality in hospitalized individuals with COVID-19: A multicenter prospective clinical study. BMC Neurology, 21 (1), 116. https://doi.org/10.1186/s12883-021-02152-5

Amer, M., Bawazeer, M., Maghrabi, K., Kamel, A. M., Butt, A., Dahhan, T., Kseibi, E., Khurshid, S. M., Abujazar, M., Alghunaim, R., Rabee, M., Abualkhair, M., Al-Janoubi, A., AlFirm, A. T., Gajic, O., Walkey, A. J., Mosier, J. M., Zabolotskikh, I. B., Gavidia, O. Y., ... Kashyap, R. (2021). Clinical Characteristics and Outcomes of Critically ill Mechanically Ventilated COVID-19 Patients Receiving interleukin-6 Receptor Antagonists and Corticosteroid Therapy: A Multicenter International Observational Study [Preprint]. Intensive Care; Critical Care Medicine. https://doi.org/10.1101/2021.04.12.21255323

Andler, R. (2019). BAISSE DE LA PRÉVALENCE DU TABAGISME QUOTIDIEN PARMI LES ADULTES : RÉSULTATS DU BAROMĖTRE DE SANTÉ PUBLIQUE FRANCE 2018 / REDUCTION OF DAILY SMOKING RATE AMONG ADULTS: RESULTS FROM THE 2018 SANTÉ PUBLIQUE FRANCE HEALTH BAROMETER. 7.

Andrade Barreto, A. P., Duarte, L. C., Cerqueira-Silva, T., Barreto Filho, M. A., Camelier, A., Tavares, N. M., Barral-Netto, M., Boaventura, V., \& Coelho Lima, M. C. (2021)Post-Acute COVID Syndrome, the Aftermath of Mild to Severe COVID-19 in Brazilian Patients [Preprint]. Infectious Diseases (except HIV/AIDS).https://doi.org/10.1101/2021.06.07.21258520

Antonio-Villa, N. E., Bello-Chavolla, O. Y., Vargas-Vazquez, A., Fermin-Martinez, C. A., Marquez-Salinas, A., \& Bahena-Lopez, J. P. (2020). Health-care workers with COVID-19 living in Mexico City: Clinical characterization and related outcomes. medRxiv, 2020.07.02.20145169. https://doi.org/10.1101/2020.07.02.20145169

Apea, V. J., Wan, Y. I., Dhairyawan, R., Puthucheary, Z. A., Pearse, R. M., Orkin, C. M., \& Prowle, J. R. (2021). Ethnicity and outcomes in patients hospitalised with COVID-19 infection in East London: An observational cohort study. BMJ Open, 11. https://doi.org/10.1136/bmjopen-2020-042140

Argenziano, M. G., Bruce, S. L., Slater, C. L., Tiao, J. R., Baldwin, M. R., Barr, R. G., Chang, B. P., Chau, K. H., Choi, J. J., Gavin, N., Goyal, P., Mills, A. M., Patel, A. A., Romney, M.-L. S., Safford, M. M., Schluger, N. W., Sengupta, S., Sobieszczyk, M. E., Zucker, J. E., ... Chen, R. (2020). Characterization and clinical course of 1000 patients with coronavirus disease 2019 in New York: Retrospective case series. BMJ, m1996.https://doi.org/10.1136/bmi.m1996

Ariza, B. E., Torres, Y. X., Salgado, D., Cepeda, M., Gomez, C., Castellanos, J. C., Suarez, F., Cuellar, A., Cardozo, C. C., Angel, J., \& Franco, M. A. (2020). Seroprevalence and seroconversion rates to SARS-CoV-2 in interns, residents, and medical doctors in a University Hospital in Bogota, Colombia. medRxiv, 2020.09.15.20195313. https://doi.org/10.1101/2020.09.15.20195313

Arleo, T., Tong, D., Shabto, J., O'Keefe, G., \& Khosroshahi, A. (2020).Clinical Course and Outcomes of coronavirus disease 2019 (COVID-19) in Rheumatic Disease Patients on Immunosuppression: A case Cohort Study at a Single Center with a Significantly Diverse Population [Preprint]. Epidemiology.https://doi.org/10.1101/2020.10.26.20219154

Asem, N., Hassany, M., Taema, K., Masoud, H., Elassal, G., Kamal, E., Amin, W., Abdelbary, A., Baki, A. A., Zaky, S., Abdalmohsen, A., Ibrahim, H., Elnady, M., Mohamed, A., Atteia, E., \& Zaid, H. (2021). Demographic and clinical features associated with in-hospital mortality in Egyptian COVID-19 patients: A retrospective cohort study.Preprint]. Infectious Diseases (except HIV/AIDS). https://doi.org/10.1101/2021.03.22.21253577

Atergeleh, H. J., Emamian, M. H., Goli, S., Rohani-Rasaf, M., Hashemi, H., \& Fotouhi, A. (2021).The risk factors of COVID-19 in a longitudinal population-based study[Preprint]. Epidemiology. https://doi.org/10.1101/2021.04.12.21255369

Auvinen, R., Nohynek, H., Syrjänen, R., Ollgren, J., Kerttula, T., Mäntylä, J., Ikonen, N., Loginov, R., Haveri, A., Kurkela, S., \& Skogberg, K. (2020). Comparison of the clinical characteristics and outcomes of hospitalized adult COVID-19 and influenza patients: A prospective observational study. medRxiv, 2020.06.29.20140632. https://doi.org/10.1101/2020.06.29.20140632 
Avouac, J., Drumez, E., Hachulla, E., Seror, R., Georgin-Lavialle, S., El Mahou, S., Pertuiset, E., Pham, T., Marotte, H., Servettaz, A., Domont, F., Chazerain, P., Devaux, M., Claudepierre, P., Langlois, V., Mekinian, A., Maria, A. T. J., Banneville, B., Fautrel, B., ... Xerri-Campano, B. (2021). COVID-19 outcomes in patients with inflammatory rheumatic and musculoskeletal diseases treated with rituximab: A cohort study. The Lancet Rheumatology, 3(6), e419-e426. https://doi.org/10.1016/S2665-9913(21)00059-X

Ayhan, M., Odabas, H., Turan, N., Ozyukseler, D. T., Kostek, O., Alkan, G., Abamor, E., \& Yildirim, M. E. (2021). Factors affecting the mortality rate of patients with cancer hospitalized with COVID19: A single center's experience. Journal of Chemotherapy, 1-10. https://doi.org/10.1080/1120009X.2021.1923153

Ayoubkhani, D., Khunti, K., Nafilyan, V., Maddox, T., Humberstone, B., Diamond, S. I., \& Banerjee, A. (2021) Epidemiology of post-COVID syndrome following hospitalisation with coronavirus: A retrospective cohort study. https://doi.org/10.1101/2021.01.15.21249885

Badr, O. I., Alwafi, H., Elrefaey, W. A., Naser, A. Y., \& Shabrawishi, M. (2021a). “Incidence and Outcomes of Pulmonary embolism among hospitalized COVID-19 patients'[Preprint]. Respiratory Medicine. https://doi.org/10.1101/2021.02.16.21251676

Badr, O. I., Alwafi, H., Elrefaey, W. A., Naser, A. Y., \& Shabrawishi, M. (2021b). "Incidence and Outcomes of Pulmonary embolism among hospitalized COVID-19 patients'[Preprint]. Respiratory Medicine. https://doi.org/10.1101/2021.02.16.21251676

Barasa, S., Kiage-Mokaya, J., Cruz-Madrid, K., \& Friedlander, M. (2020).Smoking increases the risk of COVID-19 positivity, while Never-smoking reduces the risk[Preprint]. Respiratory Medicine. https://doi.org/10.1101/2020.11.30.20241380

Barchuk, A., Skougarevskiy, D., Titaev, K., Shirokov, D., Raskina, Y., Novkunkskaya, A., Talantov, P., Isaev, A., Pomerantseva, E., Zhikrivetskaya, S., Barabanova, L., \& Volkov, V. (2021). Seroprevalence of SARS-CoV-2 antibodies in Saint Petersburg, Russia: A population-based study. Scientific Reports, 11(1), 12930. https://doi.org/10.1038/s41598-021-92206-y

Bark, D., Dhillon, N., St-Jean, M., Kinniburgh, B., McKee, G., \& Choi, A. (2021) SARS-CoV-2 transmission in K-12 schools in the Vancouver Coastal Health Region: A descriptive epidemiologic study [Preprint]. Public; Global Health.https://doi.org/10.1101/2021.05.15.21257271

Basse, C., Diakite, S., Servois, V., Frelaut, M., Noret, A., Bellesoeur, A., Moreau, P., Massiani, M.-A., Bouyer, A.-S., Vuagnat, P., Malak, Sa., Bidard, F.-C., Vanjak, D., Kriegel, I., Burnod, A., Bilger, G., Ramtohul, T., Dhonneur, G., Bouleuc, C., ... Cottu, P. (2020). Characteristics and outcome of SARS-CoV-2 infection in cancer patients. medRxiv, 2020.05.14.20101576. https://doi.org/10.1101/2020.05.14.20101576

Batty, G. D., Deary, I., Luciano, M., Altschul, D., Kivimaki, M., \& Gale, C. (2020). Psychosocial factors and hospitalisations for COVID-19: Prospective cohort study of the general population. medRxiv, 2020.05.29.20100735. https://doi.org/10.1101/2020.05.29.20100735

Bell, M. L., Catalfamo, C. J., Farland, L. V., Ernst, K. C., Jacobs, E. T., Klimentidis, Y. C., Jehn, M., \& Pogreba-Brown, K. (2021)Post-acute sequelae of COVID-19 in a non-hospitalized cohort: Results from the Arizona CoVHORT [Preprint]. Public; Global Health. https://doi.org/10.1101/2021.03.29.21254588

Bellan, M., Patti, G., Hayden, E., Azzolina, D., Pirisi, M., Acquaviva, A., Aimaretti, G., Aluffi Valletti, P., Angilletta, R., Arioli, R., Avanzi, G. C., Avino, G., Balbo, P. E., Baldon, G., Baorda, F., Barbero, E., Baricich, A., Barini, M., Barone-Adesi, F., ... Sainaghi, P. P. (2020). Fatality rate and predictors of mortality in an Italian cohort of hospitalized COVID-19 patients. Scientific Reports, $10(1), 20731$. https://doi.org/10.1038/s41598-020-77698-4

Bello-Chavolla, O. Y., Antonio-Villa, N. E., Vargas-Vázquez, A., Fermín-Martínez, C. A., Márquez-Salinas, A., \& Bahena-López, J. P. (2020). Profiling pre-symptomatic and asymptomatic cases with confirmed SARS-CoV-2 infection in Mexico City. medRxiv, 2020.07.02.20145516. https://doi.org/10.1101/2020.07.02.20145516

Bello-Chavolla, O. Y., Bahena-López, J. P., Antonio-Villa, N. E., Vargas-Vázquez, A., González-Díaz, A., Márquez-Salinas, A., Fermín-Martínez, C. A., Naveja, J. J., \& Aguilar-Salinas, C. A. (2020). Predicting Mortality Due to SARS-CoV-2: A Mechanistic Score Relating Obesity and Diabetes to COVID-19 Outcomes in Mexico. The Journal of Clinical Endocrinology \& Metabolism, 105(8). https://doi.org/10.1210/clinem/dgaa346

Benaim, A. R., Sobel, J. A., Almog, R., Lugassy, S., Shabbat, T. B., Johnson, A., Eytan, D., \& Behar, J. A. (2020)At the dawn of winter: Comparing COVID-19 and influenza presentation and trajectory [Preprint]. Infectious Diseases (except HIV/AIDS).https://doi.org/10.1101/2020.11.19.20235077

Benowitz, N. L., Schultz, K. E., Haller, C. A., Wu, A. H. B., Dains, K. M., \& Jacob, P. (2009). Prevalence of smoking assessed biochemically in an urban public hospital: A rationale for routine cotinine screening. American Journal of Epidemiology, 170(7), 885-891. https://doi.org/10.1093/aje/kwp215

Berlin, I., Thomas, D., Le Faou, A.-L., \& Cornuz, J. (2020). COVID-19 and Smoking.Nicotine \& Tobacco Research. https://doi.org/10.1093/NTR/NTAA059

Bermejo-Martin, J. F., González-Rivera, M., Almansa, R., Micheloud, D., Tedim, A. P., Domínguez-Gil, M., Resino, S., Martín-Fernández, M., Ryan Murua, P., Pérez-García, F., Tamayo, L., LopezIzquierdo, R., Bustamante, E., Aldecoa, C., Gómez, J. M., Rico-Feijoo, J., Orduña, A., Méndez, R., Fernández Natal, I., ... Kelvin, D. J. (2020). Viral RNA load in plasma is associated with critical illness and a dysregulated host response in COVID-19. Critical Care, 24(1), 691. https://doi.org/10.1186/s13054-020-03398-0

Bernaola, N., Mena, R., Bernaola, A., Lara, A., Carballo, C., Larranaga, P., \& Bielza, C. (2020). Observational Study of the Efficiency of Treatments in Patients Hospitalized with Covid-19 in Madrid. medRxiv, 2020.07.17.20155960. https://doi.org/10.1101/2020.07.17.20155960

Bertuzzi, A. F., Ciccarelli, M., Marrari, A., Gennaro, N., Dipasquale, A., Giordano, L., Cariboni, U., Quagliuolo, V. L., Alloisio, M., \& Santoro, A. (2021). Impact of active cancer on COVID-19 survival: A matched-analysis on 557 consecutive patients at an Academic Hospital in Lombardy, Italy. British Journal of Cancer. https://doi.org/10.1038/s41416-021-01396-9

Berumen, J., Schmulson, M., Alegre, J., Guerrero, G., Olaiz, G., Wong-Chew, R. M., Larriva-Sahd, J., Cantu-Brito, C., Ochoa-Guzman, A., Garcilazo-Avila, A., Gonzalez-Carballo, C., \& Chiquete, E. (2020). Risk of infection and hospitalization by Covid-19 in Mexico: A case-control study. medRxiv, 2020.05.24.20104414. https://doi.org/10.1101/2020.05.24.20104414

Best, J. H., Mohan, S. V., Kong, A. M., Patel, K., Pagel, J. M., Ivanov, B., Brawley, O. W., Jariwala-Parikh, K., Zazzali, J. L., \& Pauk, J. (2020). Baseline Demographics and Clinical Characteristics Among 3471 US Patients Hospitalized with COVID-19 and Pulmonary Involvement: A Retrospective Study. Advances in Therapy, 37(12), 4981-4995. https://doi.org/10.1007/s12325-020-01510-y 
Bhaskaran, K., Bacon, S., Evans, S. J., Bates, C. J., Rentsch, C. T., MacKenna, B., Tomlinson, L., Walker, A. J., Schultze, A., Morton, C. E., Grint, D., Mehrkar, A., Eggo, R. M., Inglesby, P., Douglas, I. J., McDonald, H. I., Cockburn, J., Williamson, E. J., Evans, D., ... Goldacre, B. (2021). Factors associated with deaths due to COVID-19 versus other causes: Population-based cohort analysis of UK primary care data and linked national death registrations within the OpenSAFELY platform. The Lancet Regional Health - Europe, 6, 100109. https://doi.org/10.1016/i.lanepe.2021.100109

Bian, H., Zheng, Z.-H., Wei, D., Zhang, Z., Kang, W.-Z., Hao, C.-Q., Dong, K., Kang, W., Xia, J.-L., Miao, J.-L., Xie, R.-H., Wang, B., Sun, X.-X., Yang, X.-M., Lin, P., Geng, J.-J., Wang, K., Cui, H.-Y., Zhang, K., ... Zhu, P. (2020). Meplazumab treats COVID-19 pneumonia: An open-labelled, concurrent controlled add-on clinical trial. medRxiv, 2020.03.21.20040691. https://doi.org/10.1101/2020.03.21.20040691

Bisso, I. C., Huespe, I., Lockhart, C., Massó, A., González Anaya, J., Hornos, M., Famiglietti, R., Di Grazia, M., Coria, P., Román, E. S., \& Heras, M. L. (2020)Clinical characteristics of critically ill patients with COVID-19 [Preprint]. Intensive Care; Critical Care Medicine.https://doi.org/10.1101/2020.12.09.20246413

Borobia, A. M., Carcas, A. J., Arnalich, F., Álvarez-Sala, R., Monserrat-Villatoro, J., Quintana, M., Figueira, J. C., Torres Santos-Olmo, R. M., García-Rodríguez, J., Martín-Vega, A., Buño, A., Ramírez, E., Martínez-Alés, G., García-Arenzana, N., Núñez, M. C., Martí-de-Gracia, M., Moreno Ramos, F., Reinoso-Barbero, F., Martin-Quiros, A., ... COVID@HULP Working Group, on behalf of the. (2020). A Cohort of Patients with COVID-19 in a Major Teaching Hospital in Europe. Journal of Clinical Medicine, 9(6), 1733. https://doi.org/10.3390/jcm9061733

Boscolo-Rizzo, P., Guida, F., Polesel, J., Marcuzzo, A. V., Capriotti, V., D’Alessandro, A., Zanelli, E., Marzolino, R., Lazzarin, C., Antonucci, P., Sacchet, E., Tofanelli, M., Borsetto, D., Gardenal, N., Pengo, M., \& Tirelli, G. (2021). Long COVID In Adults at 12 Months After Mild-to-Moderate SARS-CoV-2 Infection[Preprint]. Infectious Diseases (except HIV/AIDS). https://doi.org/10.1101/2021.04.12.21255343

Boulware, D. R., Pullen, M. F., Bangdiwala, A. S., Pastick, K. A., Lofgren, S. M., Okafor, E. C., Skipper, C. P., Nascene, A. A., Nicol, M. R., Abassi, M., Engen, N. W., Cheng, M. P., LaBar, D., Lother, S. A., MacKenzie, L. J., Drobot, G., Marten, N., Zarychanski, R., Kelly, L. E., ... Hullsiek, K. H. (2020). A Randomized Trial of Hydroxychloroquine as Postexposure Prophylaxis for Covid-19. New England Journal of Medicine. https://doi.org/10.1056/NEJMoa2016638

Bowyer, R. C. E., Varsavsky, T., Sudre, C. H., Murray, B. A. K., Freidin, M. B., Yarand, D., Ganesh, S., Capdevila, J., Thompson, E. J., Bakker, E., Cardoso, M. J., Davies, R., Wolf, J., Spector, T. D., Ourselin, S., Steves, C. J., \& Menni, C. (2020). Geo-social gradients in predicted COVID-19 prevalence and severity in Great Britain: Results from 2,266,235 users of the COVID-19 Symptoms Tracker app. https://doi.org/10.1101/2020.04.23.20076521

Boyd, S., \& Martin-Loeches, I. (2021). The incidence of venous thromboembolism in critically ill patients with COVID-19 compared with critically ill non-COVID patients.Jr J Med Sci, NA. https://doi.org/10.1007/s11845-020-02503-0

Brake, S. J., Barnsley, K., Lu, W., McAlinden, K. D., Eapen, M. S., \& Sohal, S. S. (2020). Smoking Upregulates Angiotensin-Converting Enzyme-2 Receptor: A Potential Adhesion Site for Novel Coronavirus SARS-CoV-2 (Covid-19). J Clin Med, 9, Page 841, 841. https://doi.org/10.3390/jcm9030841

Brown, J. (2020). The Smoking Toolkit Study, 2020. https://docs.google.com/presentation/d/e/2PACX-1vQmMeD0wPM7iEawo39m2QZnVBOo2e8YIQPim2R0OgYBvKhBbYanarSn5vxyk3-

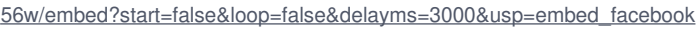

Bruce, E., Carter, B., Quinn, T. J., Verduri, A., Pearson, O., Vilches-Moraga, A., Price, A., McGovern, A., Evans, L., McCarthy, K., Hewitt, J., Moug, S., Myint, P. K., behalf of COPE Study Team, Einarsson, A., Fleck, A., Bisset, C., Alexander, R., Guaraldi, G., ... Stechman, M. (2021). Multiple House Occupancy is Associated with Mortality in Hospitalised Patients with Covid-19. European Journal of Public Health, ckab085. https://doi.org/10.1093/eurpub/ckab085

Buitrago-Garcia, D., Egli-Gany, D., Counotte, M. J., Hossmann, S., Imeri, H., Ipekci, A. M., Salanti, G., \& Low, N. (2020). Occurrence and transmission potential of asymptomatic and presymptomatic SARS-CoV-2 infections: A living systematic review and meta-analysis. PLOS Medicine, 17(9), e1003346. https://doi.org/10.1371/journal.pmed.1003346

Burrell, A. J., Pellegrini, B., Salimi, F., Begum, H., Broadley, T., Campbell, L. T., Cheng, A. C., Cheung, W., Cooper, D. J., Earnest, A., Erickson, S. J., French, C. J., Kaldor, J. M., Litton, E., Murthy, S., McAllister, R., Nichol, A., Palermo, A., Plummer, M., ... Udy, A. (2020). Outcomes of COVID-19 patients admitted to Australian intensive care units during the early phase of the pandemic. The Medical Journal of Australia, 1. https://doi.org/10.5694/mja2.50883

Bürkner, P.-C. (2017). Advanced Bayesian Multilevel Modeling with the R Package brms.arXiv:1705.11123 [Stat]. http://arxiv.org/abs/1705.11123

Byttebier, G., Belmans, L., Alexander, M., Saxberg, B. E. H., De Spiegeleer, B., De Spiegeleer, A., Devreker, N., Van Praet, J. T., Vanhove, K., Reybrouck, R., Wynendaele, E., \& Fedson, D. S. (2021). Hospital mortality in COVID-19 patients in Belgium treated with statins, ACE inhibitors and/or ARBs. Human Vaccines \& Immunotherapeutics, 1-10. https://doi.org/10.1080/21645515.2021.1920271

Cadegiani, F. A., McCoy, J., Wambier, C. G., \& Goren, A. (2020).5-Alpha-Reductase Inhibitors Reduce Remission Time of COVID-19: Results From a Randomized Double Blind Placebo Controlled Interventional Trial in 130 SARS-CoV-2 Positive Men [Preprint]. Infectious Diseases (except HIV/AIDS).https://doi.org/10.1101/2020.11.16.20232512

Caglar, A., \& Kacer, I. (2021). Anxiety levels in patients admitted to the emergency department with myocardial infarction or COVID-19 pneumoniaPSYCHOL HEALTH MED, NA, 1-9. https://doi.org/10.1080/13548506.2021.1876893

Cai, G. (2020). Bulk and Single-Cell Transcriptomics Identify Tobacco-Use Disparity in Lung Gene Expression of ACE2, the Receptor of 2019-nCov https://doi.org/10.20944/preprints202002.0051.v3; http://web.archive.org/web/20200925104805/https://www.preprints.org/manuscript/202002.0051/v3

Cai, H., Yang, L., Lu, Y., Zhang, S., Ye, C., Zhang, X., Yu, G., Gu, J., Lian, J., Hao, S., Hu, J., Zhang, Y., Jin, C., Sheng, J., Yang, Y., \& Jia, H. (2021). High body mass index is a significant risk factor for the progression and prognosis of imported COVID-19: A multicenter, retrospective cohort study. BMC Infect Dis, 21. https://doi.org/10.1186/s12879-021-05818-0

Caliskan, T., \& Saylan, B. (2020). Smoking and comorbidities are associated with COVID-19 severity and mortality in 565 patients treated in Turkey: A retrospective observational studyRev Assoc Med Bras, 66, 1679-1684. https://doi.org/10.1590/1806-9282.66.12.1679 
Campbell, J. R., Dion, C., Uppal, A., Yansouni, C. P., \& Menzies, D. (2021).Systematic Testing for SARS-CoV-2 Infection Among Essential Workers in Montréal, Canada: A Prospective Observational and Cost Assessment Study [Preprint]. Infectious Diseases (except HIV/AIDS).https://doi.org/10.1101/2021.05.12.21256956

Carrat, F., Lamballerie, X. de, Rahib, D., Blanche, H., Lapidus, N., Artaud, F., Kab, S., Renuy, A., Edelenyi, F. S. de, Meyer, L., Lydie, N., Charles, M.-A., Ancel, P.-Y., Jusot, F., Rouquette, A., Priet, S., Villaroel, P. M. S., Fourie, T., Lusivika-Nzinga, C., ... Zins, M. (2020). Seroprevalence of SARS-CoV-2 among adults in three regions of France following the lockdown and associated risk factors: A multicohort study. medRxiv, 2020.09.16.20195693. https://doi.org/10.1101/2020.09.16.20195693

Carrillo-Vega, M. F., Salinas-Escudero, G., Garcia-Peña, C., Gutierrez-Robledo, L. M., \& Parra-Rodriguez, L. (2020). Early estimation of the risk factors for hospitalisation and mortality by COVID-19 in Mexico. medRxiv, 2020.05.11.20098145. https://doi.org/10.1101/2020.05.11.20098145

CDCMMWR. (2020). Preliminary Estimates of the Prevalence of Selected Underlying Health Conditions Among Patients with Coronavirus Disease 2019 - United States, February 12-March 28, 2020. MMWR. Morbidity and Mortality Weekly Report, 69. https://doi.org/10.15585/mmwr.mm6913e2

Cen, Y., Chen, X., Shen, Y., Zhang, X.-H., Lei, Y., Xu, C., Jiang, W.-R., Xu, H.-T., Chen, Y., Zhu, J., Zhang, L.-L., \& Liu, Y.-H. (2020). Risk factors for disease progression in patients with mild to moderate coronavirus disease 2019—a multi-centre observational study. Clinical Microbiology and Infection, S1198743X20303414. https://doi.org/10.1016/i.cmi.2020.05.041

Chalkias, A., Pantazopoulos, I., Papagiannakis, N., Skoulakis, A., Laou, E., Kolonia, K., Ntalarizou, N., Ragias, D., Kampolis, C., García de Guadiana Romualdo, L., Tourlakopoulos, K., Pagonis, A., Hayek, S. S., Eugen-Olsen, J., Gourgoulianis, K., \& Arnaoutoglou, E. (2021). Clinical course and outcomes of critically ill COVID-19 patients in two successive pandemic waves[Preprint]. Intensive Care; Critical Care Medicine. https://doi.org/10.1101/2021.02.26.21251848

Chand, S., Kapoor, S., Orsi, D., Fazzari, M. J., Tanner, T. G., Umeh, G. C., Islam, M., \& Dicpinigaitis, P. V. (2020). COVID-19-Associated Critical IIIness-Report of the First 300 Patients Admitted to Intensive Care Units at a New York City Medical Center: Journal of Intensive Care Medicine. https://doi.org/10.1177/0885066620946692

Chaudhary, A., Singh, U. N., Paudel, P., Thapa, N., Khadka, K., Sah, P. K., Kamar, S. B., Joshi, J., Ansari, K. H., Tiwari, S. R., Sharma, S., Jaiswal, S. K., Joshi, R., Baskota, S., Tiwari, A. P., \& Pandey, H. R. (2020). Characteristics and outcomes of hospitalized adults with COVID-19 in Nepal: A multicenter, prospective cohort study. medRxiv, 2020.10.03.20206128. https://doi.org/10.1101/2020.10.03.20206128

Chaudhry, F., Bulka, H., Rathnam, A. S., Said, O. M., Lin, J., Lorigan, H., Bernitsas, E., Rube, J., Korzeniewski, S. J., Memon, A. B., Levy, P. D., Javed, A., Lisak, R., \& Cerghet, M. (2020). COVID19 in Multiple Sclerosis Patients and Risk Factors for Severe Infection. medRxiv, 2020.05.27.20114827. https://doi.org/10.1101/2020.05.27.20114827

Chauhan, L., Pattee, J., Ford, J., Thomas, C., Lesteberg, K., Richards, E., Loi, M., Dumont, L., Annen, K., Berg, M., Zirbes, M., Miller, A., Jenkins, T. C., Bennett, T. D., Monkowski, D., Boxer, R. S., \& Beckham, J. D. (2021). A Multi-center, Prospective, Observational-cohort controlled study of Clinical Outcomes following COVID-19 Convalescent plasma therapy in hospitalized COVID-19 patients [Preprint]. Infectious Diseases (except HIV/AIDS). https://doi.org/10.1101/2021.06.14.21258910

Chauhan, N. K., Shadrach, B. J., Garg, M. K., Bhatia, P., Bhardwaj, P., Gupta, M. K., Dutt, N., Jalandra, R. N., Garg, P., Nag, V. L., Sharma, P., Bohra, G. K., Kumar, D., Elhence, P. A., Banerjee, M., Mathur, D., Purohit, A. H., Gadepalli, R., Sureka, B., \& Misra, S. (2021). Predictors of Clinical Outcomes in Adult COVID-19 Patients Admitted to a Tertiary Care Hospital in India: An analytical crosssectional study: Predictors of clinical outcomes in COVID-19. Acta Biomedica Atenei Parmensis, 92(3), e2021024. https://doi.org/10.23750/abm.v92i3.10630

Chen, C., Jiang, J., Xu, X., Hu, Y., Hu, Y., \& Zhao, Y. (2020). Dynamic liver function indexes monitoring and clinical characteristics in three types of COVID-19 patientsmedRxiv, 2020.05.13.20099614. https://doi.org/10.1101/2020.05.13.20099614

Chen, H., Varatharajah, Y., Ramirez, S. S. de, Arnold, P., Frankenberger, C., Hota, B., \& Iyer, R. (2020)A Retrospective Longitudinal Study of COVID-19 as Seen by a Large Urban Hospital in Chicago [Preprint]. Infectious Diseases (except HIV/AIDS).https://doi.org/10.1101/2020.11.29.20240606

Chen, L., Yu, J., He, W., Chen, L., Yuan, G., Dong, F., Chen, W., Cao, Y., Yang, J., Cai, L., Wu, D., Ran, Q., Li, L., Liu, Q., Ren, W., Gao, F., Wang, H., Chen, Z., Gale, R. P., ... Hu, Y. (2020). Risk factors for death in 1859 subjects with COVID-19. Leukemia, 1-11. https://doi.org/10.1038/s41375-020-0911-0

Chen, S., Zheng, T., Wang, S., Yu, Y., Wang, P., Song, Y., \& Jiang, J. (2021). Association between risk of venous thromboembolism and mortality in patients with COVID-19/nternational Journal of Infectious Diseases, 108, 543-549. https://doi.org/10.1016/j.ijid.2021.06.005

Chen, T., Wu, D., Chen, H., Yan, W., Yang, D., Chen, G., Ma, K., Xu, D., Yu, H., Wang, H., Wang, T., Guo, W., Chen, J., Ding, C., Zhang, X., Huang, J., Han, M., Li, S., Luo, X., ... Ning, Q. (2020). Clinical characteristics of 113 deceased patients with coronavirus disease 2019: Retrospective study. BMJ, 368. https://doi.org/10.1136/bmj.m1091

Chetboun, M., Raverdy, V., Labreuche, J., Simonnet, A., Wallet, F., Caussy, C., Antonelli, M., Artigas, A., Goma, G., Meziani, F., Helms, J., Mylonakis, E., Levy, M. M., Kalligeros, M., Latronico, N., Piva, S., Cerf, C., Neuville, M., Klouche, K., ... The "LICORNE Lille Intensive Care COVID-19, Obesity Study Group", the "COVID-19 Toulouse ICU Network", the "Hasharon Hospital-Rabin Medical Center Covid-19, Obesity Study Group", the "ID, ICU Covid-19 Study Group", the "Rouen Covid-19, Obesity Study", the "COVID-O-HCL Consortium", the "MICU Lapeyronie", the "Bronx COVID-19, Obesity Study Group", the "MIR Amiens Covid19", the "Strasbourg NHC", the "Foch COVID-19 Study Group". (2021). BMI and pneumonia outcomes in critically ill COVID-19 patients: An international multicenter study. Obesity, oby.23223. https://doi.org/10.1002/oby.23223

Cho, E. R., Slutsky, A. S., \& Jha, P. (2020). Smoking and the risk of COVID-19 infection in the UK Biobank Prospective StudymedRxiv, 2020.05.05.20092445. https://doi.org/10.1101/2020.05.05.20092445

Chudasama, Y. V., Zaccardi, F., Gillies, C. L., Razieh, C., Yates, T., Kloecker, D. E., Rowlands, A. V., Davies, M. J., Islam, N., Seidu, S., Forouhi, N. G., \& Khunti, K. (2020)Patterns of Multimorbidity and Risk of Severe SARS-CoV-2 Infection: An observational study in the U.K [Preprint]. Epidemiology. https://doi.org/10.1101/2020.10.21.20216721

Chuo, C.-Y., Yau, V., Madhavan, S., Tsai, L., \& Chia, J. (2021). Risk of Severe COVID-19 Outcomes Among Patients with Rheumatoid Arthritis in the United States[Preprint]. Epidemiology. https://doi.org/10.1101/2021.07.09.21260106

Clavario, P., De Marzo, V., Lotti, R., Barbara, C., Porcile, A., Russo, C., Beccaria, F., Bonavia, M., Bottaro, L. C., Caltabellotta, M., Chioni, F., Santangelo, M., Hautala, A. J., Ameri, P., Canepa, M., \& 
Porto, I. (2020). Assessment of functional capacity with cardiopulmonary exercise testing in non-severe COVID-19 patients at three months follow-u\$Preprint]. Cardiovascular Medicine. https://doi.org/10.1101/2020.11.15.20231985

CMMID COVID-19 working group, Jarvis, C. I., Van Zandvoort, K., Gimma, A., Prem, K., Klepac, P., Rubin, G. J., \& Edmunds, W. J. (2020). Quantifying the impact of physical distance measures on the transmission of COVID-19 in the UK. BMC Medicine, 18(1), 124. https://doi.org/10.1186/s12916-020-01597-8

Colaneri, M., Novelli, V., Cutti, S., Muzzi, A., Resani, G., Monti, M. C., Rona, C., Grugnetti, A. M., Rettani, M., Rovida, F., Zuccaro, V., Triarico, A., \& Marena, C. (2021). The experience of the health care workers of a severely hit SARS-CoV-2 referral Hospital in Italy: Incidence, clinical course and modifiable risk factors for COVID-19 infection. Journal of Public Health, 43(1), 26-34. https://doi.org/10.1093/pubmed/fdaa195

Collard, D., Nurmohamed, N. S., Kaiser, Y., Reeskamp, L. F., Dormans, T., Moeniralam, H., Simsek, S., Douma, R. A., Eerens, A., Reidinga, A. C., Elbers, P., Beudel, M., Vogt, L., Stroes, E. S. G., \& Born, B.-J. H. van den. (2020). Cardiovascular risk factors are independently associated with COVID-19 mortality: A prospective cohort study[Preprint]. Infectious Diseases (except HIV/AIDS). https://doi.org/10.1101/2020.10.01.20205229

Concha-Mejia, A., \& Rincon-Sanchez, R. A. (2020). CCOFEE-GI Study: Colombian COVID19 First Experience in Gastroentrology. Characterization of digestive manifestations in patients diagnosed with COVID-19 at a highly complex institution in Bogota D.C., Colombia. medRxiv, 2020.07.24.20161604. https://doi.org/10.1101/2020.07.24.20161604

Conti, C., Marco, A. de, Mastromarino, P., Tomao, P., \& Santoro, M. G. (1999). Antiviral Effect of Hyperthermic Treatment in Rhinovirus InfectionAntimicrobial Agents and Chemotherapy, 43(4), 822-829. https://www.ncbi.nlm.nih.gov/pmc/articles/PMC89212/

Covid-19 in pregnancy, Vousden, N., Bunch, K., Morris, E., Simpson, N., Gale, C., O’Brien, P., Quigley, M., Brocklehurst, P., Kurinczuk, J. J., \& Knight, M. (2021).The incidence, characteristics and outcomes of pregnant women hospitalized with symptomatic and asymptomatic SARS-CoV-2 infection in the UK from March to September 2020: A national cohort study using the UK Obstetric Surveillance System (UKOSS). https://doi.org/10.1101/2021.01.04.21249195

Crooks, C. J., West, J., Fogarty, A., Morling, J. R., Grainge, M. J., Gonem, S., Simmonds, M., Race, A., Juurlink, I., Briggs, S., Cruikshank, S., Hammond-Pears, S., \& Card, T. R. (2020)Predicting the need for escalation of care or death from repeated daily clinical observations and laboratory results in patients with SARS-CoV-2 during 2020: A retrospective population-based cohort study from the United Kingdom. https://doi.org/10.1101/2020.12.14.20248181

Crovetto, F., Crispi, F., Llurba, E., Figueras, F., Gomez-Roig, M. D., \& Gratacos, E. (2020). SEROPREVALENCE AND CLINICAL SPECTRUM OF SARS-CoV-2 INFECTION IN THE FIRST VERSUS THIRD TRIMESTER OF PREGNANCY. medRxiv, 2020.06.17.20134098. https://doi.org/10.1101/2020.06.17.20134098

Cummins, L., Ebyarimpa, I., Cheetham, N., Brown, V. T., Brennan, K., \& Panovska-Griffiths, J. (2021).Factors associated with COVID-19 related hospitalisation, critical care admission and mortality using linked primary and secondary care data. https://doi.org/10.1101/2021.01.19.20241844

Dai, M., Tao, L., Chen, Z., Tian, Z., Guo, X., Allen-Gipson, D. S., Tan, R., Li, R., Chai, L., Ai, F., \& Liu, M. (2020). Influence of Cigarettes and Alcohol on the Severity and Death of COVID-19: A Multicenter Retrospective Study in Wuhan, China. Front Physiol, 11. https://doi.org/10.3389/fphys.2020.588553

Dambha-Miller, H., Hinton, W., Joy, M., Feher, M., \& Lusignan, S. de. (2021).Mortality in COVID-19 amongst women on Hormone Replacement Therapy or Combined Oral Contraception: A cohort study [Preprint]. Epidemiology. https://doi.org/10.1101/2021.02.16.21251853

Dashti, H., Roche, E. C., Bates, D. W., Mora, S., \& Demler, O. (2021). SARS2 simplified scores to estimate risk of hospitalization and death among patients with COVID-19Scientific Reports, 11(1), 4945. https://doi.org/10.1038/s41598-021-84603-0

Daugherty, S. E., Guo, Y., Heath, K., Dasmariñas, M. C., Jubilo, K. G., Samranvedhya, J., Lipsitch, M., \& Cohen, K. (2021).SARS-CoV-2 infection and risk of clinical sequelae during the post-acute phase: A retrospective cohort study [Preprint]. Epidemiology. https://doi.org/10.1101/2021.03.12.21253448

Dayem Ullah, A. Z. M., Sivapalan, L., Kocher, H. M., \& Chelala, C. (2021). COVID-19 in patients with hepatobiliary and pancreatic diseases: A single-centre cross-sectional study in East London. BMJ Open, 11(4), e045077. https://doi.org/10.1136/bmjopen-2020-045077

De Santi, M., Diotallevi, A., \& Brandi, G. (2021). Seroprevalence of Severe Acute Respiratory Syndrome Coronavirus-2 (SARS-CoV-2) infection in an Italian cohort in Marche Region, ItalyActa Biomedica Atenei Parmensis, 92(1), e2021070. https://doi.org/10.23750/abm.v92i1.10847.

Demichev, V., Tober-Lau, P., Nazarenko, T., Aulakh, S. K., Whitwell, H., Lemke, O., Röhl, A., Freiwald, A., Mittermaier, M., Szyrwiel, L., Ludwig, D., Correia-Melo, C., Lippert, L. J., Helbig, E. T., Stubbemann, P., Olk, N., Thibeault, C., Grüning, N.-M., Blyuss, O., ... Ralser, M. (2021). A proteomic survival predictor for COVID-19 patients in intensive care[Preprint]. Infectious Diseases (except HIV/AIDS). https://doi.org/10.1101/2021.06.24.21259374

Denholm, J. T., Gordon, C. L., Johnson, P. D., Hewagama, S. S., Stuart, R. L., Aboltins, C., Jeremiah, C., Knox, J., Lane, G. P., Tramontana, A. R., Slavin, M. A., Schulz, T. R., Richards, M., Birch, C. J., \& Cheng, A. C. (2010). Hospitalised adult patients with pandemic (H1N1) 2009 influenza in Melbourne, Australia. Medical Journal of Australia, 192(2), 84-86. https://doi.org/10.5694/j.13265377.2010.tb03424.x

Desgranges, F., Tadini, E., Munting, A., Regina, J., Filippidis, P., Viala, B., Karachalias, E., Suttels, V., Haefliger, D., Kampouri, E., Van Singer, M., Tschopp, J., Stettler, L. R., Schaad, S., Brahier, T., Hugli, O., Chabloz, Y. M., Gouveia, A., Opota, O., ... the RegCOVID research group. (2021). Post-COVID-19 syndrome in outpatients: A cohort study[Preprint]. Infectious Diseases (except HIV/AIDS). https://doi.org/10.1101/2021.04.19.21255742

Dev, N., Meena, R. C., Gupta, D. K., Gupta, N., \& Sankar, J. (2021). Risk factors and frequency of COVID-19 among healthcare workers at a tertiary care centre in India: A case-control study. Transactions of The Royal Society of Tropical Medicine and Hygiene, 115(5), 551-556. https://doi.org/10.1093/trstmh/trab047

Didikoglu, A., Maharani, A., Pendleton, N., Canal, M. M., \& Payton, A. (2021). Early life factors and COVID-19 infection in England: A prospective analysis of UK Biobank participantsEarly Hum Dev, 155. https://doi.org/10.1016/.earlhumdev.2021.105326 
Díez-Manglano, J., Marquínez, M. N. S., García, A. Á., Alcalá-Rivera, N., Riesco, I. M., Aseguinolaza, M. G., Pérez, J. L. B., Bailon, M. M., Ruiz, A. E. L.-I., Gómez, M. G., Cilleros, C. M., Fontan, P. M. P., Vázquez, L. A., Encinar, J. C. B., Boixeda, R., Sánchez, R. G., Peña Fernández, A. de la, Amigo, J. L., Sevilla, J. E., ... for the SEMI-COVID-19 Network. (2020). Healthcare workers hospitalized due to COVID-19 have no higher risk of death than general population. Data from the Spanish SEMI-COVID-19 Registry [Preprint]. Infectious Diseases (except HIV/AIDS). https://doi.org/10.1101/2020.11.23.20236810

Docherty, A. B., Harrison, E. M., Green, C. A., Hardwick, H. E., Pius, R., Norman, L., Holden, K. A., Read, J. M., Dondelinger, F., Carson, G., Merson, L., Lee, J., Plotkin, D., Sigfrid, L., Halpin, S., Jackson, C., Gamble, C., Horby, P. W., Nguyen-Van-Tam, J. S., ... Semple, M. G. (2020). Features of 20133 UK patients in hospital with covid-19 using the ISARIC WHO Clinical Characterisation Protocol: Prospective observational cohort study. BMJ, 369. https://doi.org/10.1136/bmj.m1985

Dong, X., Cao, Y., Lu, X., Zhang, J., Du, H., Yan, Y., Akdis, C. A., \& Gao, Y. (2020). Eleven faces of coronavirus disease 2019 Allergy, 75(7), 1699-1709. https://doi.org/10.1111/all.14289

Drozd, M., Pujades-Rodriguez, M., Lillie, P. J., Straw, S., Morgan, A. W., Kearney, M. T., Witte, K. K., \& Cubbon, R. M. (2021). Non-communicable disease, sociodemographic factors, and risk of death from infection: A UK Biobank observational cohort study. The Lancet Infectious Diseases, S1473309920309786. https://doi.org/10.1016/S1473-3099(20)30978-6

Duan, L., Zhang, S., Guo, M., Zhou, E., Fan, J., Wang, X., Wang, L., Wu, F., \& Jin, Y. (2020). Epidemiological and clinical characteristics in patients with SARS-CoV-2 antibody negative probable COVID-19 in Wuhan. medRxiv, 2020.06.18.20134619. https://doi.org/10.1101/2020.06.18.20134619

Dupraz, J., Butty, A., Duperrex, O., Estoppey, S., Faivre, V., Thabard, J., Zuppinger, C., Greub, G., Pantaleo, G., Pasquier, J., Rousson, V., Egger, M., Steiner-Dubuis, A., Vassaux, S., Masserey, E., Bochud, M., Nusslé, S. G., \& D’Acremont, V. (2020). Prevalence of SARS-CoV-2 in household members and other close contacts of COVID-19 cases: A serologic study in canton of Vaud, Switzerland [Preprint]. Epidemiology.https://doi.org/10.1101/2020.11.27.20239244

Durstenfeld, M. S., Sun, K., Ma, Y., Rodriguez, F., Secemsky, E. A., Parikh, R. V., \& Hsue, P. Y. (2021).Impact of HIV Infection on COVID-19 Outcomes Among Hospitalized Adults in the U.S. [Preprint]. HIV/AIDS. https://doi.org/10.1101/2021.04.05.21254938

Dye, J. A., \& Adler, K. B. (1994). Occasional review Effects of cigarette smoke on epithelial cells of the respiratory tract.Thorax, 49, 825-834. https://doi.org/10.1136/thx.49.8.825

Ebinger, J., Botwin, G. J., Albert, C. M., Alotaibi, M., Arditi, M., Berg, A. H., Binek, A., Botting, P. G., Fert-Bober, J., Figueiredo, J. C., Grein, J. D., Hasan, W., Henglin, M., Hussain, S. K., Jain, M., Joung, S., Karin, M., Kim, E. H., Li, D., ... Cheng, S. (2020). SARS-CoV-2 Seroprevalence Across a Diverse Cohort of Healthcare Workers. medRxiv, 2020.07.31.20163055. https://doi.org/10.1101/2020.07.31.20163055

Ebrahimian, S., Homayounieh, F., Rockenbach, M. A. B. C., Putha, P., Raj, T., Dayan, I., Bizzo, B. C., Buch, V., Wu, D., Kim, K., Li, Q., Digumarthy, S. R., \& Kalra, M. K. (2021). Artificial intelligence matches subjective severity assessment of pneumonia for prediction of patient outcome and need for mechanical ventilation: A cohort study. Sci. Rep, 11. https://doi.org/10.1038/s41598-020-79470-

Egede, C., Dawson, A. Z., Walker, R. J., Garacci, E., Campbell, J. A., \& Egede, L. E. (2021). Relationship between mental health diagnoses and COVID-19 test positivity, hospitalization, and mortality in Southeast Wisconsin. Psychological Medicine, 1-9. https://doi.org/10.1017/S0033291721002312

Eiros, R., Barreiro-Perez, M., Martin-Garcia, A., Almeida, J., Villacorta, E., Perez-Pons, A., Merchan, S., Torres-Valle, A., Sanchez-Pablo, C., Gonzalez-Calle, D., Perez-Escurza, O., Toranzo, I., Diaz-Pelaez, E., Fuentes-Herrero, B., Macias-Alvarez, L., Oliva-Ariza, G., Lecrevisse, Q., Fluxa, R., Bravo-Grandez, J. L., ... Sanchez, P. L. (2020). Pericarditis and myocarditis long after SARS-CoV2 infection: A cross-sectional descriptive study in health-care workers. medRxiv, 2020.07.12.20151316. https://doi.org/10.1101/2020.07.12.20151316

Elezkurtaj, S., Greuel, S., Ihlow, J., Michaelis, E., Bischoff, P., Kunze, C. A., Sinn, B. V., Gerhold, M., Hauptmann, K., Ingold-Heppner, B., Miller, F., Herbst, H., Corman, V. M., Martin, H., Heppner, F. L., \& Horst, D. (2020). Causes of Death and Comorbidities in Patients with COVID-19. medRxiv, 2020.06.15.20131540. https://doi.org/10.1101/2020.06.15.20131540

Elliott, J. H., Turner, T., Clavisi, O., Thomas, J., Higgins, J. P. T., Mavergames, C., \& Gruen, R. L. (2014). Living Systematic Reviews: An Emerging Opportunity to Narrow the Evidence-Practice Gap. PLoS Medicine, 11(2). https://doi.org/10.1371/journal.pmed.1001603

Elmunzer, B. J., Spitzer, R. L., Foster, L. D., Merchant, A. A., Howard, E. F., Patel, V. A., West, M. K., Qayad, E., Nustas, R., Zakaria, A., Piper, M. S., Taylor, J. R., Jaza, L., Forbes, N., Chau, M., Lara, L. F., Papachristou, G. I., Volk, M. L., Hilson, L. G., ... Nitchie, H. (2020). Digestive Manifestations in Patients Hospitalized with COVID-19. medRxiv, 2020.07.07.20143024. https://doi.org/10.1101/2020.07.07.20143024

El-Solh, A. A., Meduri, U. G., Lawson, Y., Carter, M., \& Mergenhagen, K. A. (2020). CLINICAL COURSE AND OUTCOME OF COVID-19 ACUTE RESPIRATORY DISTRESS SYNDROME: DATA FROM A NATIONAL REPOSITORY. medRxiv, 2020.10.16.20214130. https://doi.org/10.1101/2020.10.16.20214130

Emami, A., Javanmardi, F., Pirbonyeh, N., \& Akbari, A. (2020). Prevalence of Underlying Diseases in Hospitalized Patients with COVID-19: A Systematic Review and Meta-AnalysisArchives of Academic Emergency Medicine, 8(1), e35.

Erber, J., Kappler, V., Haller, B., Mijočević, H., Galhoz, A., Costa, C. P. da, Gebhardt, F., Graf, N., Hoffmann, D., Thaler, M., Lorenz, E., Roggendorf, H., Kohlmayer, F., Henkel, A., Menden, M. P., Ruland, J., Spinner, C. D., Protzer, U., Knolle, P., ... on behalf of the SeCoMRI Study Group. (2020). Strategies for infection control and prevalence of anti-SARS-CoV-2 IgG in 4,554 employees of a university hospital in Munich, Germany [Preprint]. Epidemiology. https://doi.org/10.1101/2020.10.04.20206136

Estiri, H., Strasser, Z. H., Klann, J. G., Naseri, P., Wagholikar, K. B., \& Murphy, S. N. (2021). Predicting COVID-19 mortality with electronic medical recordsNpj Digit. Med, 4. https://doi.org/10.1038/s41746-021-00383-x

Eugen-Olsen, J., Altintas, I., Tingleff, J., Stauning, M., Gamst-Jensen, H., Lindstroem, M. B., Rasmussen, L. J. H., Kristiansen, K. T., Rasmussen, C., Nehlin, J., Kallemose, T., \& Andersen, O. (2020). Low levels of the prognostic biomarker suPAR are predictive of mild outcome in patients with symptoms of COVID-19 - a prospective cohort study. medRxiv, 2020.05 .27 .20114678$. https://doi.org/10.1101/2020.05.27.20114678

Fan, X., Yin, C., Wang, J., Yang, M., Ma, H., Jin, G., Song, M., Hu, Z., Shen, H., \& Hang, D. (2020). Pre-diagnostic circulating concentrations of insulin-like growth factor-1 and risk of COVID-19 
mortality: Results from UK Biobank. medRxiv, 2020.07.09.20149369. https://doi.org/10.1101/2020.07.09.20149369

Farsalinos, K., Barbouni, A., \& Niaura, R. (2020). Systematic review of the prevalence of current smoking among hospitalized COVID-19 patients in China: Could nicotine be a therapeutic option? Internal and Emergency Medicine. https://doi.org/10.1007/s11739-020-02355-7

Farsalinos, K., Niaura, R., Le Houezec, J., Barbouni, A., Tsatsakis, A., Kouretas, D., Vantarakis, A., \& Poulas, K. (2020). Editorial: Nicotine and SARS-CoV-2: COVID-19 may be a disease of the nicotinic cholinergic system. Toxicology Reports. https://doi.org/10.1016/i.toxrep.2020.04.012

Favara, D. M., McAdam, K., Cooke, A., Bordessa-Kelly, A., Budriunaite, I., Bossingham, S., Houghton, S., Doffinger, R., Ainsworth, N., \& Corrie, P. G. (2020a) SARS-CoV-2 antigen and antibody prevalence among UK staff working with cancer patients during the COVID-19 pandemic [Preprint]. Oncology. https://doi.org/10.1101/2020.09.18.20197590

Favara, D. M., McAdam, K., Cooke, A., Bordessa-Kelly, A., Budriunaite, I., Bossingham, S., Houghton, S., Doffinger, R., Ainsworth, N., \& Corrie, P. G. (2020b) SARS-CoV-2 antigen and antibody prevalence among UK staff working with cancer patients during the COVID-19 pandemic [Preprint]. Oncology. https://doi.org/10.1101/2020.09.18.20197590

Faverio, P., Luppi, F., Rebora, P., Busnelli, S., Stainer, A., Catalano, M., Parachini, L., Monzani, A., Galimberti, S., Bini, F., Bodini, B. D., Betti, M., Giacomi, F. D., Scarpazza, P., Oggionni, E., Scartabellati, A., Bilucaglia, L., Ceruti, P., Modina, D., ... Pesci, A. (2021). Six-month pulmonary impairment after severe COVID-19: A prospective, multicenter follow-up study[Preprint]. Respiratory Medicine. https://doi.org/10.1101/2021.03.29.21254151

Feldman, C., \& Anderson, R. (2013). Cigarette smoking and mechanisms of susceptibility to infections of the respiratory tract and other organ systemsJ. Infect, 67, 169-184. https://doi.org/10.1016/j.jinf.2013.05.004

Feng, Y., Ling, Y., Bai, T., Xie, Y., Huang, J., Li, J., Xiong, W., Yang, D., Chen, R., Lu, F., Lu, Y., Liu, X., Chen, Y., Li, X., Li, Y., Summah, H. D., Lin, H., Yan, J., Zhou, M., ... Qu, J. (2020). COVID-19 with Different Severities: A Multicenter Study of Clinical Features. American Journal of Respiratory and Critical Care Medicine, 201(11), 1380-1388. https://doi.org/10.1164/rccm.202002-04450C

Fernandez-Fuertes, M., Corma-Gomez, A., Torres, E., Rodriguez-Pineda, E., Fuentes-Lopez, A., Rincon, P., Fernanddez, N., Garcia, F., Bernal, S., Real, L. M., Macias, J., \& Pineda, J. A. (2021). Incidence of and factors associated with SARS-CoV-2 infection among people living with HIV in Southern Spairpreprint]. HIV/AIDS. https://doi.org/10.1101/2021.03.20.21253397

Ferrari, B. L., Ferreira, C. G., Menezes, M., De Marchi, P., Canedo, J., Melo, A. C. de, Jacome, A. A., Reinert, T., Paes, R. D., Sodre, B., Barrios, C. H., \& Dienstmann, R. (2021). Determinants of COVID-19 Mortality in Patients With Cancer From a Community Oncology Practice in Brazil. JCO Glob Oncol, 7, 46-55. https://doi.org/10.1200/GO.20.00444

Feuth, T., Saaresranta, T., Karlsson, A., Valtonen, M., Peltola, V., Rintala, E., \& Oksi, J. (2020). Is sleep apnoea a risk factor for Covid-19? Findings from a retrospective cohort studymedRxiv, 2020.05.14.20098319. https://doi.org/10.1101/2020.05.14.20098319

Fillmore, N. R., La, J., Szalat, R. E., Tuck, D. P., Nguyen, V., Yildirim, C., Do, N. V., Brophy, M. T., \& Munshi, N. C. (2020). Prevalence and outcome of Covid-19 infection in cancer patients: A national VA study. medRxiv, 2020.08.21.20177923. https://doi.org/10.1101/2020.08.21.20177923

Fisher, B. A., Veenith, T., Slade, D., Gaskell, C., Rowland, M., Whitehouse, T., Scriven, J., Parekh, D., Balasubramaniam, M. S., Cooke, G., Morley, N., Gabriel, Z., Wise, M. P., Porter, J., McShane, H., Ho, L.-P., Newsome, P. N., Rowe, A., Sharpe, R., ... Kearns, P. (2021). Namilumab or infliximab compared to standard of care in hospitalised patients with COVID-19 (CATALYST): A phase 2 randomised adaptive trial [Preprint]. Intensive Care; Critical Care Medicine.https://doi.org/10.1101/2021.06.02.21258204

Fisman, D., Greer, A. L., \& Tuite, A. (2020). Derivation and Validation of Clinical Prediction Rule for COVID-19 Mortality in Ontario, CanadamedRxiv, 2020.06.21.20136929. https://doi.org/10.1101/2020.06.21.20136929

Fogh, K., Eriksen, A. R., Hasselbalch, R. B., Kristensen, E. S., Bundgaard, H., Nielsen, S. D., Jørgensen, C. S., Scharff, B. F., Erikstrup, C., Sækmose, S. G., Holm, D. K., Aagaard, B., Norsk, J., Nielsen, P. B., Kristensen, J. H., Østergaard, L., Ellermann-Eriksen, S., Andersen, B., Nielsen, H., ... Iversen, K. (2021). Seroprevalence of SARS-CoV-2 antibodies in social housing areas in Denmark [Preprint]. Epidemiology.https://doi.org/10.1101/2021.05.07.21256725

Fond, G., Pauly, V., Orleans, V., Antonini, F., Fabre, C., Sanz, M., Klay, S., Jimeno, M.-T., Leone, M., Lancon, C., Auquier, P., \& Boyer, L. (2020). Increased in-hospital mortality from COVID-19 in patients with schizophrenia. L'Encéphale, S0013700620301883. https://doi.org/10.1016/j.encep.2020.07.003

Fontanet, A., Tondeur, L., Madec, Y., Grant, R., Besombes, C., Jolly, N., Pellerin, S. F., Ungeheuer, M.-N., Cailleau, I., Kuhmel, L., Temmam, S., Huon, C., Chen, K.-Y., Crescenzo, B., Munier, S., Demeret, C., Grzelak, L., Staropoli, I., Bruel, T., ... Hoen, B. (2020). Cluster of COVID-19 in northern France: A retrospective closed cohort study. medRxiv, 2020.04.18.20071134. https://doi.org/10.1101/2020.04.18.20071134

Fox, T. A., Troy-Barnes, E., Kirkwood, A. A., Chan, W. Y., Day, J., Chavda, S. J., Kumar, E. A., David, K., Tomkins, O., Sanchez, E., Scully, M., Khwaja, A., Lambert, J., Singer, M., Roddie, C., Morris, E. C., Yong, K. L., Thomson, K. J., \& Ardeshna, K. M. (n.d.). Clinical outcomes and risk factors for severe COVID-19 infection in patients with haematological disorders receiving chemo- or immunotherapy. British Journal of Haematology, n/a(n/a). https://doi.org/10.1111/bih.17027

Freites, D., Leon, L., Mucientes, A., Rodriguez-Rodriguez, L., Font, J., Madrid, A., Colomer, J. I., Jover, J. A., Fernandez-Gutierrez, B., \& Abasolo, L. (2020). Risk factors for hospital admission related to COVID-19 in inflammatory rheumatic diseases. medRxiv, 2020.05.14.20101584. https://doi.org/10.1101/2020.05.14.20101584

Fung, H. F., Martinez, L., Alarid-Escudero, F., Salomon, J. A., Studdert, D. M., Andrews, J. R., Goldhaber-Fiebert, J. D., \& Group, S.-C. M. (2020). The household secondary attack rate of SARSCoV-2: A rapid review. Clinical Infectious Diseases. https://doi.org/10.1093/cid/ciaa1558

Gaitán-Duarte, H. G., Álvarez-Moreno, C., Rincón-Rodríguez, C. J., Yomayusa-González, N., Cortés, J. A., Villar, J. C., Bravo-Ojeda, J. S., García-Peña, Á., Adarme-Jaimes, W., Rodríguez-Romero, V. A., Villate-Soto, S. L., Buitrago, G., Chacón-Sarmiento, J., Macías-Quintero, M., Vaca, C. P., Gómez-Restrepo, C., \& Rodríguez-Malagón, N. (2021). Effectiveness of Rosuvastatin plus Colchicine, Emtricitabine/Tenofovir and a combination of them in Hospitalized Patients with SARS Covid-19 [Preprint]. Infectious Diseases (except HIV/AIDS).https://doi.org/10.1101/2021.07.06.21260085 
https://doi.org/10.1101/2020.11.11.20230052

Gallichotte, E. N., Quicke, K. M., Sexton, N. R., Fitzmeyer, E., Young, M. C., Janich, A. J., Dobos, K., Pabilonia, K. L., Gahm, G., Carlton, E. J., Ebel, G. D., \& Ehrhart, N. (2020). Longitudinal Surveillance for SARS-CoV-2 Among Staff in Six Colorado Long-Term Care Facilities: Epidemiologic, Virologic and Sequence Analysis. medRxiv, 2020.06.08.20125989. https://doi.org/10.1101/2020.06.08.20125989

Galluzzi, F., Rossi, V., Bosetti, C., \& Garavello, W. (2021). Risk Factors for Olfactory and Gustatory Dysfunctions in Patients with SARS-CoV-2 InfectionNeuroepidemiology, 55(2), 154-161. https://doi.org/10.1159/000514888

Garassino, M. C., Whisenant, J. G., Huang, L.-C., Trama, A., Torri, V., Agustoni, F., Baena, J., Banna, G., Berardi, R., Bettini, A. C., Bria, E., Brighenti, M., Cadranel, J., De Toma, A., Chini, C., Cortellini, A., Felip, E., Finocchiaro, G., Garrido, P., ... Horn, L. (2020). COVID-19 in patients with thoracic malignancies (TERAVOLT): First results of an international, registry-based, cohort study. The Lancet Oncology, 21(7), 914-922. https://doi.org/10.1016/S1470-2045(20)30314-4

García-Abellán, J., Padilla, S., Fernández-González, M., García, J. A., Agulló, V., Andreo, M., Ruiz, S., Galiana, A., Gutiérrez, F., \& Masiá, M. (2021) Long-term clinical, virological and immunological outcomes in patients hospitalized for COVID-19: Antibody response predicts long COVID [Preprint]. Infectious Diseases (except HIV/AIDS).

https://doi.org/10.1101/2021.03.08.21253124

Garibaldi, B. T., Fiksel, J., Muschelli, J., Robinson, M. L., Rouhizadeh, M., Nagy, P., Gray, J. H., Malapati, H., Ghobadi-Krueger, M., Niessen, T. M., Kim, B. S., Hill, P. M., Ahmed, M. S., Dobkin, E. D., Blanding, R., Abele, J., Woods, B., Harkness, K., Thiemann, D. R., ... Gupta, A. (2020). Patient trajectories and risk factors for severe outcomes among persons hospitalized for COVID-19 in the Maryland/DC region. medRxiv, 2020.05.24.20111864. https://doi.org/10.1101/2020.05.24.20111864

Ge, H., Zhu, M., Du, J., Zhou, Y., Wang, W., Zhang, W., Jiang, H., Qiao, Z., Gu, Z., Li, F., \& Pu, J. (2020). Cardiac Structural and Functional Characteristics in Patients with Coronavirus Disease 2019: A Serial Echocardiographic Study. medRxiv, 2020.05.12.20095885. https://doi.org/10.1101/2020.05.12.20095885

Gebhard, C. E., Sütsch, C., Bengs, S., Deforth, M., Buehler, K. P., Hamouda, N., Meisel, A., Schuepbach, R. A., Zinkernagel, A. S., Brugger, S. D., Acevedo, C., Patriki, D., Wiggli, B., Beer, J. H., Friedl, A., Twerenbold, R., Kuster, G. M., Pargger, H., Tschudin-Sutter, S., ... Gebhard, C. (2021). Sex- and Gender-specific Risk Factors of Post-COVID-19 Syndrome: A Population-based Cohort Study in Switzerland [Preprint]. Infectious Diseases (except HIV/AIDS). https://doi.org/10.1101/2021.06.30.21259757

Genecand, C., Mongin, D., Koegler, F., Lebowitz, D., Regard, S., Nehme, M., Braillard, O., Grira, M., Joubert, D., Chopard, P., Delaporte, E., Stirnemann, J., Guessous, I., Tardin, A., \& Courvoisier, D. S. (2021). Cohort profile: Actionable Register of Geneva Out- and inpatients with SARS-CoV-2 (ARGOS)[Preprint]. Public; Global Health. https://doi.org/10.1101/2021.05.24.21256813

Gerhards, C., Thiaucourt, M., Kittel, M., Becker, C., Ast, V., Hetjens, M., Neumaier, M., \& Haselmann, V. (2021). Longitudinal assessment of anti-SARS-CoV-2 antibody dynamics and clinical features following convalescence from a COVID-19 infection. International Journal of Infectious Diseases, 107, 221-227. https://doi.org/10.1016/i.ijid.2021.04.080

Gégout petit, A., Jeulin, H., Legrand, K., Bochnakian, A., Vallois, P., Schvoerer, E., \& Guillemin, F. (2021).Seroprevalence of SARS-CoV-2, symptom profiles and seroneutralization during the first COVID-19 wave in a suburban area, France. https://doi.org/10.1101/2021.02.10.21250862

Ghani, H., Navarra, A., Pyae, P. K., Mitchell, H., Evans, W., Cama, R., Shaw, M., Critchlow, B., Vaghela, T., Schechter, M., Nordin, N., Barlow, A., \& Vancheeswaran, R. (2021)Relevance of prediction scores derived from the SARS-CoV-2 first wave, in the UK COVID-19 second wave, for early discharge, severity and mortality: A PREDICT COVID UK prospective observational cohort study [Preprint]. Infectious Diseases (except HIV/AIDS).https://doi.org/10.1101/2021.06.09.21258602

Gharebaghi, N., Farshid, S., Boroofeh, B., Nejadrahim, R., Mousavi, J., Dindarian, S., \& Mohammadi, S. (2021). Evaluation of epidemiology, clinical features, prognosis, diagnosis and treatment outcomes of patients with COVID-19 in West Azerbaijan Province. International Journal of Clinical Practice, 75(6). https://doi.org/10.1111/ijcp.14108

Ghinai, I., Davis, E. S., Mayer, S., Toews, K.-A., Huggett, T. D., Snow-Hill, N., Perez, O., Hayden, M. K., Tehrani, S., Landi, A. J., Crane, S., Bell, E., Hermes, J.-M., Desai, K., Godbee, M., Jhaveri, N., Borah, B., Cable, T., Sami, S., ... Layden, J. E. (2020). Risk Factors for Severe Acute Respiratory Syndrome Coronavirus 2 Infection in Homeless Shelters in Chicago, Illinois—-March-May, 2020. Open Forum Infectious Diseases, 7(11), ofaa477. https://doi.org/10.1093/ofid/ofaa477

Giacomelli, A., Ridolfo, A. L., Milazzo, L., Oreni, L., Bernacchia, D., Siano, M., Bonazzetti, C., Covizzi, A., Schiuma, M., Passerini, M., Piscaglia, M., Coen, M., Gubertini, G., Rizzardini, G., Cogliati, C., Brambilla, A. M., Colombo, R., Castelli, A., Rech, R., ... Galli, M. (2020). 30-day mortality in patients hospitalized with COVID-19 during the first wave of the Italian epidemic: A prospective cohort study. Pharmacological Research, 158, 104931. https://doi.org/10.1016/j.phrs.2020.104931

Gianfrancesco, M. A., Leykina, L. A., Izadi, Z., Taylor, T., Sparks, J. A., Harrison, C., Trupin, L., Rush, S., Schmajuk, G., Katz, P., Jacobsohn, L., Hsu, T. Y., D’Silva, K. M., Serling-Boyd, N., Wallwork, R., Todd, D. J., Bhana, S., Costello, W., Grainger, R., ... Zell, J. (2020). Race/ethnicity association with COVID-19 outcomes in rheumatic disease: Data from the COVID-19 Global Rheumatology Alliance Physician Registry. Arthritis \& Rheumatology, art.41567. https://doi.org/10.1002/art.41567

Gianfrancesco, M., Hyrich, K. L., Al-Adely, S., Carmona, L., Danila, M. I., Gossec, L., Izadi, Z., Jacobsohn, L., Katz, P., Lawson-Tovey, S., Mateus, E. F., Rush, S., Schmajuk, G., Simard, J., Strangfeld, A., Trupin, L., Wysham, K. D., Bhana, S., Costello, W., ... Robinson, P. C. (2020). Characteristics associated with hospitalisation for COVID-19 in people with rheumatic disease: Data from the COVID-19 Global Rheumatology Alliance physician-reported registry. Annals of the Rheumatic Diseases, 79(7), 859-866. https://doi.org/10.1136/annrheumdis-2020-217871

Giannini, S., Passeri, G., Tripepi, G., Sella, S., Fusaro, M., Arcidiacono, G., Torres, M. O., Michielin, A., Prandini, T., Baffa, V., Aghi, A., Egan, C. G., Brigo, M., Zaninotto, M., Plebani, M., Vettor, R., Fioretto, P., Rossini, M., Vignali, A., ... Bertoldo, F. (2021). Effectiveness of In-Hospital Cholecalciferol Use on Clinical Outcomes in Comorbid COVID-19 Patients: A Hypothesis-Generating Study. Nutrients, 13. https://doi.org/10.3390/nu13010219

Giannouchos, T., Sussman, R., Mier, J. M., Poulas, K., \& Farsalinos, K. (2020). Characteristics and risk factors for COVID-19 diagnosis and adverse outcomes in Mexico: An analysis of 89,756 laboratory-confirmed COVID-19 cases. medRxiv, 2020.06.04.20122481. https://doi.org/10.1101/2020.06.04.20122481

Gil, S., Filho, W. J., Shinjo, S. K., Ferriolli, E., Busse, A. L., Avelino-Silva, T. J., Longobardi, I., Oliveira, G. N. de, Swinton, P., Gualano, B., Roschel, H., Bonfá, E., Utiyama, E., Segurado, A., Perondi, B., Morais, A. M., Montal, A., Letaif, L., Fusco, S., ... Francisco, M. C. P. B. (2021). Muscle Strength and Muscle Mass as Predictors of Hospital Length of Stay in Patients with Moderate to Severe 
COVID-19: A Prospective Observational Study [Preprint]. Epidemiology. https://doi.org/10.1101/2021.03.30.21254578

Gil-Agudo, A., Rodriguez-Cola, M., Jimenez-Velasco, I., Gutierrez-Henares, F., Lopez-Dolado, E., Gambarrutta-Malfatti, C., \& Vargas-Baquero, E. (2020). Clinical features of coronavirus disease 2019 (COVID-19) in a cohort of patients with disability due to spinal cord injury. medRxiv, 2020.04.20.20072918. https://doi.org/10.1101/2020.04.20.20072918

Girardeau, Y., Gallois, Y., De Bonnecaze, G., Escudé, B., Lafont, C., Chattelier, G., \& Marx, M. (2020).Confirmed central olfactory system lesions on brain MRI in COVID-19 patients with anosmia: A case-series. https://doi.org/10.1101/2020.07.08.20148692

Gold, J. A. W., Wong, K. K., Szablewski, C. M., Patel, P. R., Rossow, J., Silva, J. da, Natarajan, P., Morris, S. B., Fanfair, R. N., Rogers-Brown, J., Bruce, B. B., Browning, S. D., Hernandez-Romieu, A. C., Furukawa, N. W., Kang, M., Evans, M. E., Oosmanally, N., Tobin-D’Angelo, M., Drenzek, C., ... Jackson, B. R. (2020). Characteristics and Clinical Outcomes of Adult Patients Hospitalized with COVID-19 - Georgia, March 2020. MMWR. Morbidity and Mortality Weekly Report 69(18), 545-550. https://doi.org/10.15585/mmwr.mm6918e1

González, F., Vielot, N. A., Sciaudone, M., Toval-Ruíz, C., Premkumar, L., Gutierrez, L., Cuadra, E. C., Blandón, P., Silva, A. M. de, Rubinstein, R., Bowman, N., Becker-Dreps, S., \& Bucardo, F. (2021). Seroepidemiology of SARS-CoV-2 infections in an urban Nicaraguan population[Preprint]. Epidemiology. https://doi.org/10.1101/2021.02.25.21252447

González, F., Zepeda, O., Toval-Ruiz, C., Matute, A., Vanegas, H., Munguia, N., Centeno, E., Reyes, Y., Svensson, L., Nordgren, J., Silva, A. M. de, Becker-Dreps, S., Premkumar, L., \& Bucardo, F. (2021). Antibody response to SARS-CoV-2 infection over six months among Nicaraguan outpatients[Preprint]. Infectious Diseases (except HIV/AIDS).https://doi.org/10.1101/2021.04.28.21256122

Gori, M., Berzuini, C., D’Elia, E., Ghirardi, A., Bernardinelli, L., Gavazzi, A., Balestrieri, G., Giammarresi, A., Trevisan, R., Di Marco, F., Bellasi, A., Amoroso, M., Raimondi, F., Novelli, L., Magro, B., Mangia, G., Lorini, F. L., Guagliumi, G., Fagiuoli, S., ... Senni, M. (2020). Exposure to Renin-Angiotensin System Inhibitors Is Associated with Reduced Mortality of Older Hypertensive Covid-19 Patients. https://doi.org/10.1101/2020.12.15.20247999

Gornyk, D., Harries, M., Glöckner, S., Strengert, M., Kerrinnes, T., Bojara, G., Castell, S., Frank, K., Gubbe, K., Heise, J.-K., Hernandez, P., Kappert, O., Kern, W., Illig, T., Klopp, N., Maaß, H., Ortmann, J., Kessel, B., Roller, G., ... Krause, G. (2021a). SARS-CoV-2 seroprevalence in Germany - a population based sequential study in five regions[Preprint]. Infectious Diseases (except HIV/AIDS). https://doi.org/10.1101/2021.05.04.21256597

Gornyk, D., Harries, M., Glöckner, S., Strengert, M., Kerrinnes, T., Bojara, G., Castell, S., Frank, K., Gubbe, K., Heise, J.-K., Hernandez, P., Kappert, O., Kern, W., Illig, T., Klopp, N., Maaß, H., Ortmann, J., Kessel, B., Roller, G., ... Krause, G. (2021b). SARS-CoV-2 seroprevalence in Germany - a population based sequential study in five regions[Preprint]. Infectious Diseases (except HIV/AIDS). https://doi.org/10.1101/2021.05.04.21256597

Govind, R., Freitas, D. F. de, Pritchard, M. R., Hayes, R. D., \& MacCabe, J. H. (2020). Clozapine treatment and risk of COVID-19medRxiv, 2020.06.17.20133595 https://doi.org/10.1101/2020.06.17.20133595

Goyal, P., Choi, J. J., Pinheiro, L. C., Schenck, E. J., Chen, R., Jabri, A., Satlin, M. J., Campion, T. R., Nahid, M., Ringel, J. B., Hoffman, K. L., Alshak, M. N., Li, H. A., Wehmeyer, G. T., Rajan, M., Reshetnyak, E., Hupert, N., Horn, E. M., Martinez, F. J., ... Safford, M. M. (2020). Clinical Characteristics of Covid-19 in New York City. New England Journal of Medicine, 382(24), 2372-2374. https://doi.org/10.1056/NEJMc2010419

Greenbaum, U., Klein, K., Martinez, F., Song, J., Thall, P. F., Ramdial, J. L., Knape, C., Aung, F. M., Scroggins, J., Knopfelmacher, A., Mulanovich, V., Borjan, J., Adachi, J., Muthu, M., Leung, C., Medina, M. C., Champlin, R., Olson, A., Alousi, A., ... Shpall, E. J. (2021). High Levels of Common Cold Coronavirus Antibodies in Convalescent Plasma Are Associated With Improved Survival in COVID-19 Patients. Frontiers in Immunology, 12, 675679. https://doi.org/10.3389/fimmu.2021.675679

Griffith, G., Morris, T. T., Tudball, M., Herbert, A., Mancano, G., Pike, L., Sharp, G. C., Palmer, T. M., Smith, G. D., Tilling, K., Zuccolo, L., Davies, N. M., \& Hemani, G. (2020). Collider bias undermines our understanding of COVID-19 disease risk and severity. medRxiv, 2020.05.04.20090506. https://doi.org/10.1101/2020.05.04.20090506

Grint, D. J., Wing, K., Williamson, E., McDonald, H. I., Bhaskaran, K., Evans, D., Evans, S. J., Walker, A. J., Hickman, G., Nightingale, E., Schultze, A., Rentsch, C. T., Bates, C., Cockburn, J., Curtis, H. J., Morton, C. E., Bacon, S., Davy, S., Wong, A. Y., ... Eggo, R. M. (2021). Case fatality risk of the SARS-CoV-2 variant of concern B.1.1.7 in England[Preprint]. Infectious Diseases (except HIV/AIDS). https://doi.org/10.1101/2021.03.04.21252528

Grundy*, E. J., Suddek*, T., Filippidis, F. T., Majeed, A., \& Coronini-Cronberg, S. (2020). Smoking, SARS-CoV-2 and COVID-19: A review of reviews considering implications for public health policy and practice. Tobacco Induced Diseases, 18(July). https://doi.org/10.18332/tid/124788

Gu, T., Mack, J. A., Salvatore, M., Sankar, S. P., Valley, T. S., Singh, K., Nallamothu, B. K., Kheterpal, S., Lisabeth, L., Fritsche, L. G., \& Mukherjee, B. G. (2020). COVID-19 outcomes, risk factors and associations by race: A comprehensive analysis using electronic health records data in Michigan Medicine. medRxiv, 2020.06.16.20133140. https://doi.org/10.1101/2020.06.16.20133140

Guan, W., Liang, W., Zhao, Y., Liang, H., Chen, Z., Li, Y., Liu, X., Chen, R., Tang, C., Wang, T., Ou, C., Li, L., Chen, P., Sang, L., Wang, W., Li, J., Li, C., Ou, L., Cheng, B., ... He, J. (2020). Comorbidity and its impact on 1590 patients with COVID-19 in China: A nationwide analysis. European Respiratory Journal, 55(5). https://doi.org/10.1183/13993003.00547-2020

Guan, W., Ni, Z., Hu, Y., Liang, W., Ou, C., He, J., Liu, L., Shan, H., Lei, C., Hui, D. S. C., Du, B., Li, L., Zeng, G., Yuen, K.-Y., Chen, R., Tang, C., Wang, T., Chen, P., Xiang, J., ... Zhong, N. (2020). Clinical Characteristics of Coronavirus Disease 2019 in China. New England Journal of Medicine. https://doi.org/10.1056/NEJMoa2002032

Guo, T., Fan, Y., Chen, M., Wu, X., Zhang, L., He, T., Wang, H., Wan, J., Wang, X., \& Lu, Z. (2020). Cardiovascular Implications of Fatal Outcomes of Patients With Coronavirus Disease 2019 (COVID-19). JAMA Cardiology, 5(7), 811-818. https://doi.org/10.1001/jamacardio.2020.1017

Gupta, R., Agrawal, R., Bukhari, Z., Jabbar, A., Wang, D., Diks, J., Alshal, M., Emechebe, D. Y., Brunicardi, F. C., Lazar, J. M., Chamberlain, R., Burza, A., \& Haseeb, M. A. (2020). Higher Comorbidities and Early Death is Characteristic of Hospitalized African-American Patients with COVID-19. medRxiv, 2020.07.15.20154906. https://doi.org/10.1101/2020.07.15.20154906

Guzmán, M. J. M., Castillo-Gonzalez, A., Gonzalez, J. L. B., Gámez, M. G., Enciso, E. A. M., Robles, I. O., Díaz, A. L. G., Peña, C. M. G., Medina, L. M., Colin, V. A. M., \& Jose Manuel, A. G. (2021). Factors associated with increased mortality in critically ill COVID-19 patients in a Mexican public hospital: The other faces of health system oversaturationPreprint]. Infectious Diseases (except HIV/AIDS). https://doi.org/10.1101/2021.03.04.21252084 
Hadi, Y. B., Naqvi, S. F. Z., Kupec, J. T., \& Sarwari, A. R. (2020). Characteristics and outcomes of COVID-19 in patients with HIV: A multicentre research network studyAIDS, 34(13), F3-F8. https://doi.org/10.1097/QAD.00000000000002666

Hadjadj, J., Yatim, N., Barnabei, L., Corneau, A., Boussier, J., Pere, H., Charbit, B., Bondet, V., Chenevier-Gobeaux, C., Breillat, P., Carlier, N., Gauzit, R., Morbieu, C., Pene, F., Marin, N., Roche, N., Szwebel, T.-A., Smith, N., Merkling, S., ... Terrier, B. (2020). Impaired type I interferon activity and exacerbated inflammatory responses in severe Covid-19 patients. medRxiv, 2020.04.19.20068015. https://doi.org/10.1101/2020.04.19.20068015

Halabi, M. E., Feghali, J., Tallón de Lara, P., Narasimhan, B., Ho, K., Saabiye, J., Huang, J., Osorio, G., Mathew, J., Wisnivesky, J., \& Steiger, D. (2021)A Novel Evidence-Based Predictor Tool for Hospitalization and Length of Stay: Insights from COVID19 Patients in New York City [Preprint]. Emergency Medicine.https://doi.org/10.1101/2021.04.24.21256042

Hamadah, H., Alahmad, B., Behbehani, M., Al-Youha, S., Almazeedi, S., Al-Haddad, M., Jamal, M. H., \& Al-Sabah, S. (2020). COVID-19 clinical outcomes and nationality: Results from a Nationwide registry in Kuwait. BMC Public Health, 20(1), 1384. https://doi.org/10.1186/s12889-020-09490-y

Hamer, M., Kivimäki, M., Gale, C. R., \& Batty, G. D. (2020). Lifestyle risk factors, inflammatory mechanisms, and COVID-19 hospitalization: A community-based cohort study of 387,109 adults in UK. Brain, Behavior, and Immunity, 87, 184-187. https://doi.org/10.1016/j.bbi.2020.05.059

Han, H., Peng, X., Zheng, F., Deng, G., Cheng, X., \& Peng, L. (2021). Association of Gender With Outcomes in Hospitalized Patients With 2019-nCoV Infection in WuhanFrontiers in Public Health, 9, 619482. https://doi.org/10.3389/fpubh.2021.619482

Hao, S.-R., Zhang, S.-Y., Lian, J.-S., Jin, X., Ye, C.-Y., Cai, H., Zhang, X.-L., Hu, J.-H., Zheng, L., Zhang, Y.-M., Jia, H.-Y., Yu, G.-D., Wang, X.-Y., Gu, J.-Q., Lu, Y.-F., Yu, X.-P., Yu, L., Xiang, D.-R., Ye, C.-Y., ... Yang, Y.-D. (2020). Liver Enzyme Elevation in Coronavirus Disease 2019: A Multicenter, Retrospective, Cross-Sectional Study. The American Journal of Gastroenterology. https://doi.org/10.14309/ajg.0000000000000717

Hausfater, P., Boutolleau, D., Lacombe, K., Beurton, A., Dumont, M., Constantin, J.-M., Ghosn, J., Combes, A., Cury, N., Guedj, R., Djibré, M., Bompard, R., Mazerand, S., Pourcher, V., Gimeno, L., Marois, C., Teyssou, E., Marcelin, A.-G., Hajage, D., \& Tubach, F. (2021). Cumulative incidence of SARS-CoV-2 infection and associated risk factors among frontline health care workers in Paris, France: The SEROCOV prospective cohort study [Preprint]. Epidemiology. https://doi.org/10.1101/2021.03.09.21253200

Heili-Frades, S. (n.d.). COVID-19 Outcomes in 4712 consecutively confirmed SARS-CoV2 cases in the city of Madrid medRxiv. https://doi.org/10.1101/2020.05.22.20109850

Hernández-Garduño, E. (2020). Obesity is the comorbidity more strongly associated for Covid-19 in Mexico. A case-control study.Obesity Research \& Clinical Practice. https://doi.org/10.1016/i.orcp.2020.06.001

Herzberg, J., Vollmer, T., Fischer, B., Becher, H., Becker, A.-K., Honarpisheh, H., Guraya, S. Y., Strate, T., \& Knabbe, C. (2021).SARS-CoV-2-antibody response in health care workers after vaccination or natural infection in a longitudinal observational study [Preprint]. Infectious Diseases (except HIV/AIDS).https://doi.org/10.1101/2021.06.09.21258648

Hewitt, J., Carter, B., Vilches-Moraga, A., Quinn, T. J., Braude, P., Verduri, A., Pearce, L., Stechman, M., Short, R., Price, A., Collins, J. T., Bruce, E., Einarsson, A., Rickard, F., Mitchell, E., Holloway, M., Hesford, J., Barlow-Pay, F., Clini, E., ... Guaraldi, G. (2020). The effect of frailty on survival in patients with COVID-19 (COPE): A multicentre, European, observational cohort study. The Lancet Public Health, 5(8), e444-e451. https://doi.org/10.1016/S2468-2667(20)30146-8

Higuchi, T., Nishida, T., Iwahashi, H., Morimura, O., Otani, Y., Okauchi, Y., Yokoe, M., Suzuki, N., Inada, M., \& Abe, K. (2020). Early Clinical Factors Predicting the Development of Critical Disease in Japanese Patients with COVID-19: A Single-Center Retrospective, Observational Study. medRxiv, 2020.07.29.20159442. https://doi.org/10.1101/2020.07.29.20159442

Hippisley-Cox, J., Young, D., Coupland, C., Channon, K. M., Tan, P. S., Harrison, D. A., Rowan, K., Aveyard, P., Pavord, I. D., \& Watkinson, P. J. (2020). Risk of severe COVID-19 disease with ACE inhibitors and angiotensin receptor blockers: Cohort study including 8.3 million people. Heart, heartjnl-2020-317393. https://doi.org/10.1136/heartjnl-2020-317393

Hirschtick, J. L., Titus, A. R., Slocum, E., Power, L. E., Hirschtick, R. E., Elliott, M. R., McKane, P., \& Fleischer, N. L. (2021). Population-Based Estimates of Post-acute Sequelae of Severe Acute Respiratory Syndrome Coronavirus 2 (SARS-CoV-2) Infection (PASC) Prevalence and Characteristics. Clinical Infectious Diseases, ciab408. https://doi.org/10.1093/cid/ciab408

Ho, F. K., Celis-Morales, C. A., Gray, S. R., Katikireddi, S. V., Niedzwiedz, C. L., Hastie, C., Ferguson, L. D., Berry, C., Mackay, D. F., Gill, J. M., Pell, J. P., Sattar, N., \& Welsh, P. (2020). Modifiable and non-modifiable risk factors for COVID-19, and comparison to risk factors for influenza and pneumonia: Results from a UK Biobank prospective cohort study. BMJ Open, 10. https://doi.org/10.1136/bmjopen-2020-040402

Ho, K. S., Narasimhan, B., Sheehan, J., Wu, L., \& Fung, J. Y. (2020). Controversy over Smoking in COVID-19 - A Real World Experience in New York CityJ Med Virol, NA. https://doi.org/10.1002/jmv.26738

Hoertel, N., Rico, M. S., Vernet, R., Beeker, N., Jannot, A.-S., Neuraz, A., Salamanca, E., Paris, N., Daniel, C., Gramfort, A., Lemaitre, G., Bernaux, M., Bellamine, A., Lemogne, C., Airagnes, G., Burgun, A., \& Limosin, F. (2020). Association between SSRI Antidepressant Use and Reduced Risk of Intubation or Death in Hospitalized Patients with Coronavirus Disease 2019: A Multicenter Retrospective Observational Study. medRxiv, 2020.07.09.20143339. https://doi.org/10.1101/2020.07.09.20143339

Hoertel, N., Rico, M. S., Vernet, R., Jannot, A.-S., Neuraz, A., Blanco, C., Lemogne, C., Airagnes, G., Paris, N., Daniel, C., Gramfort, A., Lemaitre, G., Bernaux, M., Bellamine, A., Beeker, N., \& Limosin, F. (2020). Observational Study of Haloperidol in Hospitalized Patients with Covid-19. medRxiv, 2020.07.15.20150490. https://doi.org/10.1101/2020.07.15.20150490

Hoertel, N., Sánchez, M., Vernet, R., Beeker, N., Neuraz, A., Alvarado, J., Daniel, C., Paris, N., Gramfort, A., Lemaitre, G., Salamanca, E., Bernaux, M., Bellamine, A., Burgun, A., \& Limosin, F. (2020). Dexamethasone use and Mortality in Hospitalized Patients with Coronavirus Disease 2019: A Multicenter Retrospective Observational Study[Preprint]. Infectious Diseases (except HIV/AIDS). https://doi.org/10.1101/2020.10.23.20218172

Hoertel, N., Sánchez-Rico, M., Gulbins, E., Kornhuber, J., Carpinteiro, A., Abellán, M., Muela, P. de la, Vernet, R., Beeker, N., Neuraz, A., Delcuze, A., Alvarado, J. M., Meneton, P., \& Limosin, F. (2021). Association between Psychotropic Medications Functionally Inhibiting Acid Sphingomyelinase and reduced risk of Intubation or Death among Individuals with Mental Disorder and Severe COVID-19: An Observational Study [Preprint]. Public; Global Health. https://doi.org/10.1101/2021.02.18.21251997 
Hoffmann, M., Kleine-Weber, H., Schroeder, S., Krüger, N., Herrler, T., Erichsen, S., Schiergens, T. S., Herrler, G., Wu, N.-H., Nitsche, A., Müller, M. A., Drosten, C., \& Pöhlmann, S. (2020). SARSCoV-2 Cell Entry Depends on ACE2 and TMPRSS2 and Is Blocked by a Clinically Proven Protease Inhibitor. Cell, 181(2), 271-280.e8. https://doi.org/10.1016/j.cell.2020.02.052

Holman, N., Knighton, P., Kar, P., O'Keefe, J., Curley, M., Weaver, A., Barron, E., Bakhai, C., Khunti, K., Wareham, N. J., Sattar, N., Young, B., \& Valabhji, J. (2020). Risk factors for COVID-19related mortality in people with type 1 and type 2 diabetes in England: A population-based cohort study. The Lancet Diabetes \& Endocrinology, O(0). https://doi.org/10.1016/S2213-8587(20)30271-0

Hopkinson, N. S., Rossi, N., Moustafa, J. E.-S., Laverty, A. A., Quint, J. K., Freydin, M. B., Visconti, A., Murray, B., Modat, M., Ourselin, S., Small, K., Davies, R., Wolf, J., Spector, T., Steves, C. J., \& Falchi, M. (2020). Current tobacco smoking and risk from COVID-19: Results from a population symptom app in over 2.4 million people. medRxiv, 2020.05.18.20105288. https://doi.org/10.1101/2020.05.18.20105288

Houlihan, C. F., Vora, N., Byrne, T., Lewer, D., Kelly, G., Heaney, J., Gandhi, S., Spyer, M. J., Beale, R., Cherepanov, P., Moore, D., Gilson, R., Gamblin, S., Kassiotis, G., McCoy, L. E., Swanton, C., Hayward, A., Nastouli, E., Aitken, J., ... Hatipoglu, E. (2020). Pandemic peak SARS-CoV-2 infection and seroconversion rates in London frontline health-care workers. The Lancet, S0140673620314847. https://doi.org/10.1016/S0140-6736(20)31484-7

HPG23 Covid-19 Study Group, Raimondi, F., Novelli, L., Ghirardi, A., Russo, F. M., Pellegrini, D., Biza, R., Trapasso, R., Giuliani, L., Anelli, M., Amoroso, M., Allegri, C., Imeri, G., Sanfilippo, C., Comandini, S., Hila, E., Manesso, L., Gandini, L., Mandelli, P., ... Di Marco, F. (2021). Covid-19 and gender: Lower rate but same mortality of severe disease in women—an observational study. BMC Pulmonary Medicine, 21(1), 96. https://doi.org/10.1186/s12890-021-01455-0

Hu, L., Chen, S., Fu, Y., Gao, Z., Long, H., Ren, H., Zuo, Y., Li, H., Wang, J., Xv, Q., Yu, W., Liu, J., Shao, C., Hao, J., Wang, C., Ma, Y., Wang, Z., Yanagihara, R., Wang, J., \& Deng, Y. (2020). Risk Factors Associated with Clinical Outcomes in 323 COVID-19 Patients in Wuhan, China. medRxiv, 2020.03.25.20037721. https://doi.org/10.1101/2020.03.25.20037721

Huang, C., Wang, Y., Li, X., Ren, L., Zhao, J., Hu, Y., Zhang, L., Fan, G., Xu, J., Gu, X., Cheng, Z., Yu, T., Xia, J., Wei, Y., Wu, W., Xie, X., Yin, W., Li, H., Liu, M., ... Cao, B. (2020). Clinical features of patients infected with 2019 novel coronavirus in Wuhan, China. The Lancet, 395(10223), 497-506. https://doi.org/10.1016/S0140-6736(20)30183-5

Huang, Y., Yang, R., Xu, Y., \& Gong, P. (2020). Clinical characteristics of 36 non-survivors with COVID-19 in Wuhan, ChinamedRxiv, 2020.02.27.20029009 https://doi.org/10.1101/2020.02.27.20029009

Hultcrantz, M., Richter, J., Rosenbaum, C., Patel, D., Smith, E., Korde, N., Lu, S., Mailankody, S., Shah, U., Lesokhin, A., Hassoun, H., Tan, C., Maura, F., Derkach, A., Diamond, B., Rossi, A., Pearse, R. N., Madduri, D., Chari, A., ... Landgren, O. (2020). COVID-19 infections and outcomes in patients with multiple myeloma in New York City: A cohort study from five academic centers. medRxiv, 2020.06.09.20126516. https://doi.org/10.1101/2020.06.09.20126516

Husain, Q., Kokinakos, K., Kuo, Y.-H., Zaidi, F., Houston, S., \& Shargorodsky, J. (2021). Characteristics of COVID-19 smell and taste dysfunction in hospitalized patientsAmerican Journal of Otolaryngology, 42(6), 103068. https://doi.org/10.1016/j.amjoto.2021.103068

Hussein, M. H., Toraih, E. A., Attia, A. S., Youssef, M., Omar, M., Burley, N., Zhang, A. D., Roos, J., Houghton, A., Aniemeka, N., Shama, M. A., Duchesne, J., \& Kandil, E. (2020). Asthma in CoVID19: An extra chain fitting around the neck? medRxiv, 2020.07.13.20153130. https://doi.org/10.1101/2020.07.13.20153130

Ibarra-Nava, I., Flores-Rodriguez, K. G., Ruiz-Herrera, V., Ochoa-Bayona, H. C., Salinas-Zertuche, A., Padilla-Orozco, M., \& Salazar-Montalvo, R. G. (2020). Ethnic disparities in COVID-19 mortality in Mexico: A cross-sectional study based on national data. medRxiv, 2020.08.26.20182543. https://doi.org/10.1101/2020.08.26.20182543

Ibrahim, D., Dulipsingh, L., Zapatka, L., Eadie, R., Crowell, R., Williams, K., Wakefield, D., Cook, L., Puff, J., \& Hussain, S. A. (2020). Factors Associated with Good Patient Outcomes Following Convalescent Plasma in COVID-19: A Prospective Phase II Clinical Trial. medRxiv, 2020.08.27.20183293. https://doi.org/10.1101/2020.08.27.20183293

Iftime, S., López-Azcona, A. F., Vallverdu, I., Hernandez-Flix, S., Febrer, G. de, Parra, S., Hernández-Aguilera, A., Riu, F., Joven, J., Camps, J., Castro, A., \& REUSCOVID Study Group. (2020)First and second waves of coronavirus disease-19: A comparative study in hospitalized patients in Reus, Spain [Preprint]. Infectious Diseases (except HIV/AIDS). https://doi.org/10.1101/2020.12.10.20246959

Ikitimur, H., Uysal, B. B., Cengiz, M., Ikitimur, B., Uysal, H., Ozcan, E., Islamoglu, M. S., Seyhan, S., Yavuzer, H., \& Yavuzer, S. (n.d.). "Determining Host Factors Contributing to Disease Severity in a Family Cluster of 29 Hospitalized SARS-CoV-2 Patients: Could Genetic Factors Be Relevant in the Clinical Course of COVID-19?". Journal of Medical Virology, n/a(n/a). https://doi.org/10.1002/imv.26106

Ilic, I., Zdravkovic, M., Timcic, S., Unic Stojanovic, D., Bojic, M., \& Loncar, G. (2021). Pneumonia in healthcare workers during a COVID-19 outbreak at a cardiovascular hospitals/nternational Journal of Infectious Diseases, 103, 188-193. https://doi.org/10.1016/i.ijid.2020.11.156

Incerti, D., Rizzo, S., Li, X., Lindsay, L., Yau, V., Keebler, D., Chia, J., \& Tsai, L. (2020).Risk factors for mortality among hospitalized patients with COVID-19[Preprint]. Infectious Diseases (except HIV/AIDS). https://doi.org/10.1101/2020.09.22.20196204

International Severe Acute Respiratory and emerging Infections Consortium, Escher, M., Hall, M., Baillie, J. K., Baruch, J., Blumberg, L., Carson, G., Citarella, B. W., Dankwa, E. A., Docherty, A., Dryden, M., Donnelly, C. A., Dunning, J., Fraser, C., Hardwick, H., Harrison, E. M., Holden, K. A., Jassat, W., Kartsonaki, C., ... Merson, L. (2020). ISARIC Clinical Data Report 10 February 2021. https://doi.org/10.1101/2020.07.17.20155218

International Severe Acute Respiratory and emerging Infections Consortium, Hall, M., Pritchard, M., Dankwa, E. A., Baillie, J. K., Carson, G., Citarella, B. W., Docherty, A., Donnelly, C. A., Dunning, J., Fraser, C., Hardwick, H., Harrison, E. M., Holden, K. A., Kartsonaki, C., Kennon, K., Lee, J., McLean, K., Openshaw, P. J. M., ... Merson, L. (2020). ISARIC Clinical Data Report 20 November 2020 [Preprint]. Infectious Diseases (except HIV/AIDS).https://doi.org/10.1101/2020.07.17.20155218

Invernizzi, A., Torre, A., Parrulli, S., Zicarelli, F., Schiuma, M., Colombo, V., Giacomelli, A., Cigada, M., Milazzo, L., Ridolfo, A., Faggion, I., Cordier, L., Oldani, M., Marini, S., Villa, P., Rizzardini, G., Galli, M., Antinori, S., Staurenghi, G., \& Meroni, L. (2020). Retinal findings in patients with COVID-19: Results from the SERPICO-19 study. EClinicalMedicine, $27,100550$. https://doi.org/10.1016/i.eclinm.2020.100550 
Ioannou, G. N., Locke, E., Green, P., Berry, K., O’Hare, A. M., Shah, J. A., Crothers, K., Eastment, M. C., Dominitz, J. A., \& Fan, V. S. (2020). Risk Factors for Hospitalization, Mechanical Ventilation, or Death Among 10131 US Veterans With SARS-CoV-2 Infection. JAMA Network Open, 3(9), e2022310. https://doi.org/10.1001/jamanetworkopen.2020.22310

Ip, A., Berry, D. A., Hansen, E., Goy, A. H., Pecora, A. L., Sinclaire, B. A., Bednarz, U., Marafelias, M., Berry, S. M., Berry, N. S., Mathura, S., Sawczuk, I. S., Biran, N., Go, R. C., Sperber, S., Piwoz, J. A., Balani, B., Cicogna, C., Sebti, R., ... Goldberg, S. L. (2020). Hydroxychloroquine and Tocilizumab Therapy in COVID-19 Patients - An Observational Study. medRxiv, 2020.05 .21 .20109207$. https://doi.org/10.1101/2020.05.21.20109207

ISARIC Clinical Characterisation Group, Baillie, J. K., Baruch, J., Beane, A., Blumberg, L., Bozza, F., Broadley, T., Burrell, A., Carson, G., Citarella, B., Dagens, A., Elotmani, L., Escher, M., Farshait, N., Garcia Barrio, N., Goffard, J.-C., Hall, M., Hashmi, M., Horby, P., ... Merson, L. (2020). ISARIC Clinical Data Report issued: 14 July 2021 [Preprint]. Infectious Diseases (except HIV/AIDS). https://doi.org/10.1101/2020.07.17.20155218

Islam, M. Z., Riaz, B. K., Islam, A. S., Khanam, F., Akhter, J., Choudhury, R., Farhana, N., Uddin, M. J., \& Efa, S. S. (2020). Risk factors associated with morbidity and mortality outcomes of COVID19 patients on the 14th and 28th day of the disease course: A retrospective cohort study in Bangladesh. medRxiv, 2020.08.17.20176586. https://doi.org/10.1101/2020.08.17.20176586

Israel, A., Feldhamer, I., Lahad, A., Levin-Zamir, D., \& Lavie, G. (2020). Smoking and the risk of COVID-19 in a large observational population studymedRxiv, 2020.06.01.20118877. https://doi.org/10.1101/2020.06.01.20118877

Israel, A., Schäffer, A. A., Cicurel, A., Feldhamer, I., Tal, A., Cheng, K., Sinha, S., Schiff, E., Lavie, G., \& Ruppin, E. (2020). Large population study identifies drugs associated with reduced COVID19 severity. medRxiv, 2020.10.13.20211953. https://doi.org/10.1101/2020.10.13.20211953

Iversen, K., Bundgaard, H., Hasselbalch, R. B., Kristensen, J. H., Nielsen, P. B., Pries-Heje, M., Knudsen, A. D., Christensen, C. E., Fogh, K., Norsk, J. B., Andersen, O., Fischer, T. K., Jensen, C. A. J., Larsen, M., Torp-Pedersen, C., Rungby, J., Ditlev, S. B., Hageman, I., Møgelvang, R., ... Ullum, H. (2020). Risk of COVID-19 in health-care workers in Denmark: An observational cohort study. The Lancet Infectious Diseases, O(0). https://doi.org/10.1016/S1473-3099(20)30589-2

Izquierdo, J. L., Almonacid, C., Gonzalez, Y., Rio-Bermudez, C. D., Ancochea, J., Cardenas, R., \& Soriano, J. B. (2020). The impact of COVID-19 on patients with asthmamedRxiv, 2020.07.24.20161596. https://doi.org/10.1101/2020.07.24.20161596

Izzi-Engbeaya, C., Distaso, W., Amin, A., Yang, W., Idowu, O., Kenkre, J. S., Shah, R. J., Woin, E., Shi, C., Alavi, N., Bedri, H., Brady, N., Blackburn, S., Leczycka, M., Patel, S., Sokol, E., TokeBjolgerud, E., Qayum, A., Abdel-Malek, M., ... Salem, V. (2020). Severe COVID-19 and Diabetes: A Retrospective Cohort Study from Three London Teaching Hospitals. medRxiv, 2020.08.07.20160275. https://doi.org/10.1101/2020.08.07.20160275

Jackson, S. E., Brown, J., Shahab, L., Steptoe, A., \& Fancourt, D. (2020). COVID-19, smoking and inequalities: A study of 53002 adults in the UK.Tobacco Control, tobaccocontrol-2020-055933. https://doi.org/10.1136/tobaccocontrol-2020-055933

Jafari, D., Gandomi, A., Makhnevich, A., Qiu, M., Rolston, D. M., Gottesman, E. P., Tsegaye, A., Mayo, P. H., Stewart, M. E., Zhang, M., \& Hajizadeh, N. (2021).Trajectories of Hypoxemia \& Respiratory System Mechanics of COVID-19 ARDS in the NorthCARDS dataset. https://doi.org/10.1101/2021.01.26.21250492

Jakob, C. E. M., Borgmann, S., Duygu, F., Behrends, U., Hower, M., Merle, U., Friedrichs, A., Tometten, L., Hanses, F., Jung, N., Rieg, S., Wille, K., Grüner, B., Klinker, H., Gersbacher-Runge, N., Hellwig, K., Eberwein, L., Dolff, S., Rauschning, D., ... Vehreschild, J. J. (2020). First results of the "Lean European Open Survey on SARS-CoV-2-Infected Patients (LEOSS)." Infection. https://doi.org/10.1007/s15010-020-01499-0

Jehi, L., Ji, X., Milinovich, A., Erzurum, S., Merlino, A., Gordon, S., Young, J. B., \& Kattan, M. W. (2020). Development and validation of a model for individualized prediction of hospitalization risk in 4,536 patients with COVID-19. PLOS ONE, 15(8), e0237419. https://doi.org/10.1371/journal.pone.0237419

Jha, R. K., Shrestha, A., Tamang, B., K. C., I., \& Sah, S. K. (2021).Predisposing factors associated with the severity of the illness in adults with Covid-19 in Nepa[Preprint]. Infectious Diseases (except HIV/AIDS). https://doi.org/10.1101/2021.05.16.21257280

Jin, C., Gu, J., Yuan, Y., Long, Q., Zhang, Q., Zhou, H., Wu, W., \& Zhang, W. (2020). Treatment of Six COVID-19 Patients with Convalescent PlasmamedRxiv, 2020.05.21.20109512. https://doi.org/10.1101/2020.05.21.20109512

Jin, X., Lian, J.-S., Hu, J.-H., Gao, J., Zheng, L., Zhang, Y.-M., Hao, S.-R., Jia, H.-Y., Cai, H., Zhang, X.-L., Yu, G.-D., Xu, K.-J., Wang, X.-Y., Gu, J.-Q., Zhang, S.-Y., Ye, C.-Y., Jin, C.-L., Lu, Y.-F., Yu, X., ... Yang, Y. (2020). Epidemiological, clinical and virological characteristics of 74 cases of coronavirus-infected disease 2019 (COVID-19) with gastrointestinal symptoms. Gut, 69(6), 10021009. https://doi.org/10.1136/gutinl-2020-320926

Jose, T., Croghan, I. T., Hays, J. T., Schroeder, D. R., \& Warner, D. O. (2021). Electronic Cigarette Use Is Not Associated with COVID-19 DiagnosisJournal of Primary Care \& Community Health 12, 215013272110243. https://doi.org/10.1177/21501327211024391

Joubert, A., Andry, F., Bertolotti, A., Accot, F., Koumar, Y., Legrand, F., Poubeau, P., Manaquin, R., Gérardin, P., \& Levin, C. (2021). Distinguishing non severe cases of dengue from COVID-19 in the context of co-epidemics: A cohort study in a SARS-CoV-2 testing center on Reunion island. PLOS Neglected Tropical Diseases, 15(4), e0008879. https://doi.org/10.1371/journal.pntd.0008879

Joubert, A., Andry, F., Bertolotti, A., Accot, F., Koumar, Y., Legrand, F., Poubeau, P., Manaquin, R., Gérardin, P., \& Levin, C. (2020).Distinguishing non severe cases of dengue from COVID-19 in the context of co-epidemics: A cohort study in a SARS-CoV-2 testing center on Reunion island [Preprint]. Infectious Diseases (except HIV/AIDS).https://doi.org/10.1101/2020.10.20.20214718

Jun, T., Nirenberg, S., Kovatch, P., \& Huang, K. (2020). Sex-specificity of mortality risk factors among hospitalized COVID-19 patients in New York City: Prospective cohort studymedRxiv, 2020.07.29.20164640. https://doi.org/10.1101/2020.07.29.20164640

Kahlert, C. R., Persi, R., Güsewell, S., Egger, T., Leal-Neto, O. B., Sumer, J., Flury, D., Brucher, A., Lemmenmeier, E., Möller, J. C., Rieder, P., Stocker, R., Vuichard-Gysin, D., Wiggli, B., Albrich, W. C., Babouee Flury, B., Besold, U., Fehr, J., Kuster, S. P., ... Kohler, P. (2021). Non-occupational and occupational factors associated with specific SARS-CoV-2 antibodies among hospital workers A multicentre cross-sectional study. Clinical Microbiology and Infection, S1198743X21002366. https://doi.org/10.1016/j.cmi.2021.05.014 
Kalan, M. E., Ghobadi, H., Taleb, Z. B., Ward, K. D., Adham, D., Matin, S., Fazlzadeh, M., \& Narimani, S. (2020). Descriptive characteristics of hospitalized adult smokers and never-smokers with COVID-19. Tobacco Induced Diseases, 18(May). https://doi.org/10.18332/tid/122759

Kantele, A., Laaveri, T., Kareinen, L., Pakkanen, S. H., Blomgren, K., Mero, S., Patjas, A., Virtanen, J., Uusitalo, R., Lappalainen, M., Jarvinen, A., Kurkela, S., Jaaskelainen, A. J., Vapalahti, O., \& Sironen, T. (2020). SARS-CoV-2 infections among healthcare workers at Helsinki University Hospital, Finland, spring 2020: Serosurvey, symptoms and risk factors. Travel Med Infect Dis, 39. https://doi.org/10.1016/j.tmaid.2020.101949

Kara Polat, A., Oguz Topal, I., Karadag, A. S., Aksoy, H., Koku Aksu, A. E., Ozkur, E., Ozkok Akbulut, T., Topaloglu Demir, F., Engin, B., Uzuncakmak, T. K., \& Kivanc Altunay, I. (2020). The impact of COVID-19 in patients with psoriasis: A multicenter study in Istanbul. Dermatol Ther, NA. https://doi.org/10.1111/dth.14691

Kibler, M., Carmona, A., Marchandot, B., Matsushita, K., Trimaille, A., Kanso, M., Dietrich, L., How-Choong, C., Odier, A., Gennesseaux, G., Schramm, O., Reydel, A. C., Kindo, M., Hoang, M., Hess, S., Sato, C., Ohlmann, S., Jesel, L., Morel, O., \& Ohlmann, P. (2020). Risk and severity of COVID-19 and ABO blood group in transcatheter aortic valve patients. medRxiv, 2020.06.13.20130211. https://doi.org/10.1101/2020.06.13.20130211

Killerby, M. E. (2020). Characteristics Associated with Hospitalization Among Patients with COVID-19 — Metropolitan Atlanta, Georgia, March-April 2020 MMWR. Morbidity and Mortality Weekly Report, 69. https://doi.org/10.15585/mmwr.mm6925e1

Kim, E. S., Chin, B. S., Kang, C. K., Kim, N. J., Kang, Y. M., Choi, J.-P., Oh, D. H., Kim, J.-H., Koh, B., Kim, S. E., Yun, N. R., Lee, J.-H., Kim, J. Y., Kim, Y., Bang, J. H., Song, K.-H., Kim, H. B., Chung, K., Oh, M., \& Covid-19, on behalf of the K. N. C. for C. M. of. (2020). Clinical Course and Outcomes of Patients with Severe Acute Respiratory Syndrome Coronavirus 2 Infection: A Preliminary Report of the First 28 Patients from the Korean Cohort Study on COVID-19. Journal of Korean Medical Science, 35(13). https://doi.org/10.3346/jkms.2020.35.e142

Kim, L., Garg, S., O’Halloran, A., Whitaker, M., Pham, H., Anderson, E. J., Armistead, I., Bennett, N. M., Billing, L., Como-Sabetti, K., Hill, M., Kim, S., Monroe, M. L., Muse, A., Reingold, A., Schaffner, W., Sutton, M., Talbot, H. K., Torres, S. M., ... Langley, G. E. (2020). Interim Analysis of Risk Factors for Severe Outcomes among a Cohort of Hospitalized Adults Identified through the U.S. Coronavirus Disease 2019 (COVID-19)-Associated Hospitalization Surveillance Network (COVID-NET). medRxiv, 2020.05.18.20103390. https://doi.org/10.1101/2020.05.18.20103390

Kimmig, L. M., Wu, D., Gold, M., Pettit, N. N., Pitrak, D., Mueller, J., Husain, A. N., Mutlu, E. A., \& Mutlu, G. M. (2020). IL-6 Inhibition in Critically III COVID-19 Patients Is Associated With Increased Secondary Infections. Frontiers in Medicine, 7, 583897. https://doi.org/10.3389/fmed.2020.583897

Kjetland, E. F., Kalleberg, K. T., Søraas, C. L., Hammarström, B., Myklebust, T. Å., Jenum, S., Axelsen, E., Lind, A., Bævre-Jensen, R., Jørgensen, S. B., Pettersen, F. O., Solberg, L. B., Hadley, C. L., Istre, M. S., Liestøl, K., Dahl, J. A., Ursin, G., \& Søraas, A. (2020). Risk factors for community transmission of SARS-CoV-2. A cross-sectional study in 116,678 people https://doi.org/10.1101/2020.12.23.20248514

Klang, E., Kassim, G., Soffer, S., Freeman, R., Levin, M. A., \& Reich, D. L. (n.d.). Morbid Obesity as an Independent Risk Factor for COVID-19 Mortality in Hospitalized Patients Younger than 50. Obesity, n/a(n/a). https://doi.org/10.1002/oby.22913

Klang, E., Soffer, S., Nadkarni, G., Glicksberg, B., Freeman, R., Horowitz, C., Reich, D. L., \& Levin, M. A. (2020). Sex Differences in Age and Comorbidities for COVID-19 Mortality in Urban New York City. SN Comprehensive Clinical Medicine, 2(9), 1319-1322. https://doi.org/10.1007/s42399-020-00430-w

Kleynhans, J., Tempia, S., Wolter, N., Gottberg, A. von, Bhiman, J. N., Buys, A., Moyes, J., McMorrow, M. L., Kahn, K., Gómez-Olivé, F. X., Tollman, S., Martinson, N. A., Wafawanaka, F., Lebina, L., Toit, J. du, Jassat, W., Neti, M., Brauer, M., Cohen, C., \& for the PHIRST-C Group. (2021). Longitudinal SARS-CoV-2 seroprevalence in a rural and urban community household cohort in South Africa, during the first and second waves July 2020-March 2021 [Preprint]. Public; Global Health. https://doi.org/10.1101/2021.05.26.21257849

Kline, J. A., Camargo, C. A., Courtney, D. M., Kabrhel, C., Nordenholz, K. E., Aufderheide, T., Baugh, J. J., Beiser, D. G., Bennett, C. L., Bledsoe, J., Castillo, E., Chisolm-Straker, M., Goldberg, E. M., House, H., House, S., Jang, T., Lim, S. C., Madsen, T. E., McCarthy, D. M., ... Wilburn, J. (2021). Clinical prediction rule for SARS-CoV-2 infection from 116 U.S. Emergency departments 2-222021. PLOS ONE, 16(3), e0248438. https://doi.org/10.1371/journal.pone.0248438

Kolin, D. A., Kulm, S., \& Elemento, O. (2020). Clinical and Genetic Characteristics of Covid-19 Patients from UK Biobank.medRxiv, 2020.05.05.20075507. https://doi.org/10.1101/2020.05.05.20075507

Kortela, E., Kirjavainen, V., Ahava, M. J., Jokiranta, S. T., But, A., Lindahl, A., Jääskeläinen, A. E., Jääskeläinen, A. J., Järvinen, A., Jokela, P., Kallio-Kokko, H., Loginov, R., Mannonen, L., Ruotsalainen, E., Sironen, T., Vapalahti, O., Lappalainen, M., Kreivi, H.-R., Jarva, H., ... Kekäläinen, E. (2020). Real-life clinical sensitivity of SARS-CoV-2 RT-PCR test in symptomatic patients [Preprint]. Infectious Diseases (except HIV/AIDS). https://doi.org/10.1101/2020.11.01.20223107

Kronbichler, A., Kresse, D., Yoon, S., Lee, K. H., Effenberger, M., \& Shin, J. I. (2020). Asymptomatic patients as a source of COVID-19 infections: A systematic review and meta-analysis. International Journal of Infectious Diseases 98, 180-186. https://doi.org/10.1016/i.jij. 2020.06.052

Kuderer, N. M., Choueiri, T. K., Shah, D. P., Shyr, Y., Rubinstein, S. M., Rivera, D. R., Shete, S., Hsu, C.-Y., Desai, A., Lima Lopes, G. de, Grivas, P., Painter, C. A., Peters, S., Thompson, M. A., Bakouny, Z., Batist, G., Bekaii-Saab, T., Bilen, M. A., Bouganim, N., ... West, J. (2020). Clinical impact of COVID-19 on patients with cancer (CCC19): A cohort study. The Lancet, 395(10241), 19071918. https://doi.org/10.1016/S0140-6736(20)31187-9

Kumar, A., Prasad, G., Srivastav, S., Gautam, V. K., \& Sharma, N. (2020). A Retrospective Study on Efficacy and Safety of Guduchi Ghan Vati for Covid-19 Asymptomatic PatientsmedRxiv, 2020.07.23.20160424. https://doi.org/10.1101/2020.07.23.20160424

Kurashima, K., Kagiyama, N., Ishiguro, T., Takaku, Y., Nakajima, H., Shibata, S., Matsui, Y., Takano, K., Isono, T., Nishida, T., Kawate, E., Hosoda, C., Kobayashi, Y., Takayanagi, N., \& Yanagisawa, T. (2020). IgG antibody seroconversion and the clinical progression of COVID-19 pneumonia: A retrospective, cohort study https://doi.org/10.1101/2020.07.16.20154088

Lamure, S., Duléry, R., Blasi, R. D., Chauchet, A., Laureana, C., Deau-Fischer, B., Drenou, B., Soussain, C., Rossi, C., Noël, N., Choquet, S., Bologna, S., Joly, B., Kohn, M., Malak, S., Fouquet, G., Daguindau, E., Bernard, S., Thiéblemont, C., ... Besson, C. (2020). Determinants of outcome in Covid-19 hospitalized patients with lymphoma: A retrospective multicentric cohort study. EClinicalMedicine, 27. https://doi.org/10.1016/j.eclinm.2020.100549 
Lan, F.-Y., Suharlim, C., Kales, S. N., \& Yang, J. (2020). Association between SARS-CoV-2 infection, exposure risk and mental health among a cohort of essential retail workers in the United States. medRxiv, 2020.06.08.20125120. https://doi.org/10.1101/2020.06.08.20125120

Lassale, C., Gaye, B., Hamer, M., Gale, C. R., \& Batty, G. D. (2020). Ethnic disparities in hospitalisation for COVID-19 in England: The role of socioeconomic factors, mental health, and inflammatory and pro-inflammatory factors in a community-based cohort study. Brain, Behavior, and Immunity, 88, 44-49. https://doi.org/10.1016/j.bbi.2020.05.074

Lassen, M. C. H., Skaarup, K. G., Sengeløv, M., Iversen, K., Ulrik, C. S., Jensen, J. U. S., \& Biering-Sørensen, T. (2021). Alcohol Consumption and the Risk of Acute Respiratory Distress Syndrome in COVID-19. Annals of the American Thoracic Society, 18(6), 1074-1076. https://doi.org/10.1513/AnnalsATS.202008-988RL

Lee, S. C., Son, K. J., Kim, D. W., Han, C. H., Choi, Y. J., Kim, S. W., \& Park, S. C. (2021). Smoking and the Risk of Severe Acute Respiratory Syndrome Coronavirus 2 (SARS-CoV-2) Infection. Nicotine \& Tobacco Research, ntab079. https://doi.org/10.1093/ntr/ntab079

Lee, S. F., Nikšić, M., Rachet, B., Sanchez, M.-J., \& Luque-Fernandez, M. A. (2021). Socioeconomic Inequalities and Ethnicity Are Associated with a Positive COVID-19 Test among Cancer Patients in the UK Biobank Cohort. Cancers, 13(7), 1514. https://doi.org/10.3390/cancers13071514

Leister, I., Ponocny-Seliger, E., Kollaritsch, H., Dungel, P., Holzer, B., Grillari, J., Redl, H., Ponocny, I., Wilfing, C., Aigner, L., Exner, M., Stainer, M., Hackl, M., Hausner, T., Mittermayr, R., \& Schaden, W. (2021). Antibody seroprevalence and rate of asymptomatic infections with SARS-CoV-2 in Austrian hospital personne/https://doi.org/10.1101/2021.02.01.21250898

Lekoubou, A., Pelton, M., Ba, D. M., \& Ssentongo, P. (2021). Racial Disparities in Ischemic Stroke Among Patients with COVID-19 in the United StatesJournal of Stroke and Cerebrovascular Diseases, 30(8), 105877. https://doi.org/10.1016/j.jstrokecerebrovasdis.2021.105877

Lenka, J., Chhabria, M. S., Sharma, N., Tan, B. E.-X., Boppana, L. K. T., Venugopal, S., \& Sondhi, D. S. (2020). Clinical characteristics and outcomes of critically ill patients with COVID-19 in a tertiary community hospital in upstate New York. medRxiv, 2020.06.18.20135046. https://doi.org/10.1101/2020.06.18.20135046

Lewnard, J. A., Mora, A. M., Nkwocha, O., Kogut, K., Rauch, S. A., Morga, N., Hernandez, S., Wong, M. P., Huen, K., Andrejko, K., Jewell, N. P., Parra, K. L., Holland, N., Harris, E., Cuevas, M., \& Eskenazi, B. (2021). Prevalence and clinical profile of SARS-CoV-2 infection among farmworkers in Monterey County, California: June-November, 2020 https://doi.org/10.1101/2020.12.27.20248894

Lévy, Y., Wiedemann, A., Hejblum, B. P., Durand, M., Lefebvre, C., Surénaud, M., Lacabaratz, C., Perreau, M., Foucat, E., Déchenaud, M., Tisserand, P., Blengio, F., Hivert, B., Gautier, M., Cervantes-Gonzalez, M., Bachelet, D., Laouénan, C., Bouadma, L., Timsit, J.-F., ... the French COVID cohort study group. (2020). CD177, a specific marker of neutrophil activation, is a hallmark of COVID-19 severity and death [Preprint]. Allergy; Immunology. https://doi.org/10.1101/2020.12.12.20246934

Li, F., Cai, Y., Gao, C., Zhou, L., Chen, R., Zhang, K., Li, W., Zhang, R., Zhang, X., Wang, D., Liu, Y., \& Tao, L. (2020)Clinical Course And Risk Factors For In-hospital Death In Critical COVID-19 In Wuhan, China [Preprint]. Public; Global Health. https://doi.org/10.1101/2020.09.26.20189522

Li, Jiong, Chen, Y., Chen, S., Wang, S., Zhang, D., Wang, J., Postmus, D., Zeng, H., Qin, G., Shen, Y., Jiang, J., \& Yu, Y. (2020). Derivation and validation of a prognostic model for predicting inhospital mortality in patients admitted with COVID-19 in Wuhan, China: The PLANS (Platelet Lymphocyte Age Neutrophil Sex) model. medRxiv, 2020.05.13.20100370. https://doi.org/10.1101/2020.05.13.20100370

Li, Jie, Li, S., Cai, Y., Liu, Q., Li, X., Zeng, Z., Chu, Y., Zhu, F., \& Zeng, F. (2020). Epidemiological and Clinical Characteristics of 17 Hospitalized Patients with 2019 Novel Coronavirus Infections Outside Wuhan, China. medRxiv, 2020.02.11.20022053. https://doi.org/10.1101/2020.02.11.20022053

Li, J., Long, X., Zhang, Q., Fang, X., Li, N., Fedorova, B., Hu, S., Li, Jh., Xiong, N., \& Lin, Z. (2020). Tobacco smoking confers risk for severe COVID-19 unexplainable by pulmonary imagingJournal of Internal Medicine, joim.13190. https://doi.org/10.1111/joim.13190

Li, Jingwen, Long, X., Zhu, C., Wang, H., Wang, T., Lin, Z., Li, J., \& Xiong, N. (n.d.). Olfactory Dysfunction in Recovered Coronavirus Disease 2019 (COVID-19) PatientsMovement Disorders, n/a(n/a). https://doi.org/10.1002/mds.28172

Li, S., Jun, T., Wang, Z., Kao, Y.-H., Schadt, E., Konig, M. F., Bettegowda, C., Vogelstein, J. T., Papadopoulos, N., Parsons, R. E., Chen, R., Schadt, E. E., Li, L., \& Oh, W. K. (2021)COVID-19 outcomes among hospitalized men with or without exposure to alpha-1-adrenergic receptor blocking agents [Preprint]. Infectious Diseases (except HIV/AIDS).

https://doi.org/10.1101/2021.04.08.21255148

Li, S., Sarangarajan, R., Jun, T., Kao, Y.-H., Wang, Z., Schadt, E., Kiebish, M. A., Granger, E., Narain, N. R., Chen, R., Schadt, E. E., \& Li, L. (2021)Association of in-hospital use of ACE-I/ARB and COVID-19 outcomes in African American population [Preprint]. Infectious Diseases (except HIV/AIDS).https://doi.org/10.1101/2021.04.14.21255443

Li, Y., Tong, C. H., Bare, L. A., \& Devlin, J. J. (2021). Assessment of the Association of Vitamin D Level With SARS-CoV-2 Seropositivity Among Working-Age Adults/AMA Network Open, 4(5), e2111634. https://doi.org/10.1001/jamanetworkopen.2021.11634

Lian, J., Jin, X., Hao, S., Cai, H., Zhang, S., Zheng, L., Jia, H., Hu, J., Gao, J., Zhang, Y., Zhang, X., Yu, G., Wang, X., Gu, J., Ye, C., Jin, C., Lu, Y., Yu, X., Yu, X., ... Yang, Y. (n.d.). Analysis of Epidemiological and Clinical Features in Older Patients With Coronavirus Disease 2019 (COVID-19) Outside Wuhan. Clinical Infectious Diseases. https://doi.org/10.1093/cid/ciaa242

Liao, Y., Feng, Y., Wang, B., Wang, H., Huang, J., Wu, Y., Wu, Z., Chen, X., Yang, C., Fu, X., \& Sun, H. (2020). Clinical Characteristics and Risk factors for developed COVID-19 patients transferring to designated hospital from Jianghan Fangcang shelter Hospital: A retrospective, observational study. medRxiv, 2020.04.21.20074724. https://doi.org/10.1101/2020.04.21.20074724

Lin, K. J., Schneeweiss, S., Tesfaye, H., D’Andrea, E., Liu, J., Lii, J., Murphy, S. N., \& Gagne, J. J. (2020). Pharmacotherapy for Hospitalized Patients with COVID-19: Treatment Patterns by Disease Severity. Drugs, 80, 1961-1972. https://doi.org/10.1007/s40265-020-01424-7

Liu, R., Ming, X., Xu, O., Zhou, J., Peng, H., Xiang, N., Zhang, J., \& Zhu, H. (2020). Association of Cardiovascular Manifestations with In-hospital Outcomes in Patients with COVID-19: A Hospital Staff Data. medRxiv, 2020.02.29.20029348. https://doi.org/10.1101/2020.02.29.20029348

Liu, W., Tao, Z.-W., Wang, L., Yuan, M.-L., Liu, K., Zhou, L., Wei, S., Deng, Y., Liu, J., Liu, H.-G., Yang, M., \& Hu, Y. (2020). Analysis of factors associated with disease outcomes in hospitalized 
patients with 2019 novel coronavirus disease. Chinese Medical Journal, 133(9), 1032-1038. https://doi.org/10.1097/CM9.0000000000000775

Lohia, P., Sreeram, K., Nguyen, P., Choudhary, A., Khicher, S., Yarandi, H., Kapur, S., \& Badr, M. S. (2021). Preexisting respiratory diseases and clinical outcomes in COVID-19: A multihospital cohort study on predominantly African American population. Respir Res, 22. https://doi.org/10.1186/s12931-021-01647-6

Lombardi, A., Mangioni, D., Consonni, D., Cariani, L., Bono, P., Cantù, A. P., Tiso, B., Carugno, M., Muscatello, A., Lunghi, G., Pesatori, A. C., Riboldi, L., Ceriotti, F., Bandera, A., \& Gori, A. (2021). Seroprevalence of anti-SARS-CoV-2 IgG among healthcare workers of a large university hospital in Milan, Lombardy, Italy: A cross-sectional study. BMJ Open, $11(2)$, e047216. https://doi.org/10.1136/bmjopen-2020-047216

Lopez-Medrano, F., Perez-Jacoiste Asin, M. A., Fernandez-Ruiz, M., Carretero, O., Lalueza, A., Maestro de la Calle, G., Caro, J. M., Calle, C. de la, Catalan, M., Garcia Garcia, R., Martinez-Lopez, J., Origuen, J., Ripoll, M., San Juan, R., Trujillo, H., Sevillano, A., Gutierrez, E., Miguel, B. de, Aguilar, F., ... Aguado, J. M. (2020). Combination therapy with tocilizumab and corticosteroids for aged patients with severe COVID-19 pneumonia: A single-center retrospective study. [Preprint]. Infectious Diseases (except HIV/AIDS).https://doi.org/10.1101/2020.09.26.20202283

Louis, S., Dhawan, A., Newey, C., Nair, D., Jehi, L., Hantus, S., \& Punia, V. (2020). Continuous Electroencephalography (cEEG) Characteristics and Acute Symptomatic Seizures in COVID-19 Patients. medRxiv, 2020.05.26.20114033. https://doi.org/10.1101/2020.05.26.20114033

Lowe, K. E., Zein, J., Hatipoglu, U., \& Attaway, A. (2021). Association of Smoking and Cumulative Pack-Year Exposure With COVID-19 Outcomes in the Cleveland Clinic COVID-19 RegistryJAMA Intern Med, NA. https://doi.org/10.1001/jamainternmed.2020.8360

Lubetzky, M., Aull, M., Craig-Shapiro, R., Lee, J., Lee, J., Sultan, S., Marku-Podvorica, J., Gingras, L., Kodiyanplakkal, R. P., Hartono, C., Saal, S., Muthukumar, T., Kapur, S., Suthanthiran, M., \& Dadhania, D. (2020). Kidney Allograft Recipients Diagnosed with Coronavirus Disease-2019: A Single Center Report. medRxiv, 2020.04.30.20086462. https://doi.org/10.1101/2020.04.30.20086462

Lucar, J., Wingler, M. J. B., Cretella, D. A., Ward, L. M., Sims Gomillia, C. E., Chamberlain, N., Shimose, L. A., Brock, J. B., Harvey, J., Wilhelm, A., Majors, L. T., Jeter, J. B., Bueno, M. X., Albrecht, S., Navalkele, B., Mena, L. A., \& Parham, J. (2021). Epidemiology, Clinical Features, and Outcomes of Hospitalized Adults with COVID-19: Early Experience from an Academic Medical Center in Mississippi. Southern Medical Journal, 114(3), 144-149. https://doi.org/10.14423/SMJ.0000000000001222

Luo, H., Liu, S., Wang, Y., Phillips-Howard, P. A., Yang, Y., Ju, S., \& Wang, D. (2020). Age differences in clinical features and outcomes in patients with COVID-19, Jiangsu, China: A retrospective, multi-center cohort study. medRxiv, 2020.06.01.20086025. https://doi.org/10.1101/2020.06.01.20086025

Luo, J., Rizvi, H., Preeshagul, I. R., Egger, J. V., Hoyos, D., Bandlamudi, C., McCarthy, C. G., Falcon, C. J., Schoenfeld, A. J., Arbour, K. C., Chaft, J. E., Daly, R. M., Drilon, A., Eng, J., lqbal, A., Lai, W. V., Li, B. T., Lito, P., Namakydoust, A., ... Hellmann, M. D. (2020). COVID-19 in patients with lung cancer. Annals of Oncology, 31(10), 1386-1396. https://doi.org/10.1016/.annonc.2020.06.007 Lusignan, S. de, Dorward, J., Correa, A., Jones, N., Akinyemi, O., Amirthalingam, G., Andrews, N., Byford, R., Dabrera, G., Elliot, A., Ellis, J., Ferreira, F., Lopez Bernal, J., Okusi, C., Ramsay, M., Sherlock, J., Smith, G., Williams, J., Howsam, G., ... Hobbs, F. D. R. (2020). Risk factors for SARS-CoV-2 among patients in the Oxford Royal College of General Practitioners Research and Surveillance Centre primary care network: A cross-sectional study. The Lancet Infectious Diseases, S1473309920303716. https://doi.org/10.1016/S1473-3099(20)30371-6

Madakkatel, I., King, C., Zhou, A., Mulugeta, A., Lumsden, A., McDonnell, M., \& Hyppönen, E. (2021).Identifying risk factors for COVID-19 severity and mortality in the UK Biobank[Preprint]. Epidemiology. https://doi.org/10.1101/2021.05.10.21256935

Madariaga, M. L. L., Guthmiller, J., Schrantz, S., Jansen, M., Christenson, C., Kumar, M., Prochaska, M., Wool, G., Durkin, A., Oh, W. H., Trockman, L., Vigneswaran, J., Keskey, R., Shaw, D. G., Dugan, H., Zheng, N., Cobb, M., Utset, H., Wang, J., ... Donington, J. (2020). Clinical predictors of donor antibody titer and correlation with recipient antibody response in a COVID-19 convalescent plasma clinical trial. medRxiv, 2020.06.21.20132944. https://doi.org/10.1101/2020.06.21.20132944

Mady, A. F., Abdulrahman, B., Ramadan, O. E., Mumtaz, S. A., Al-Odat, M. A., Kuhail, A., Altoraifi, R., Alshae, R., Alharthy, A. M., Karakitsos, D., \& Aletreby, W. Th. (2021)Effect of Tocilizumab on "ventilator free days" composite outcome in SARS-CoV-2 patients. A retrospective competing risk analysis [Preprint]. Intensive Care; Critical Care Medicine. https://doi.org/10.1101/2021.04.01.21254794

Magagnoli, J., Narendran, S., Pereira, F., Cummings, T. H., Hardin, J. W., Sutton, S. S., \& Ambati, J. (2020). Outcomes of Hydroxychloroquine Usage in United States Veterans Hospitalized with COVID-19. Med, S2666634020300064. https://doi.org/10.1016/.j.medj.2020.06.001

Magleby, R., Westblade, L. F., Trzebucki, A., Simon, M. S., Rajan, M., Park, J., Goyal, P., Safford, M. M., \& Satlin, M. J. (n.d.). Impact of SARS-CoV-2 Viral Load on Risk of Intubation and Mortality Among Hospitalized Patients with Coronavirus Disease 2019. Clinical Infectious Diseases. https://doi.org/10.1093/cid/ciaa851

Makaronidis, J., Mok, J., Balogun, N., Magee, C. G., Omar, R. Z., Carnemolla, A., \& Batterham, R. L. (2020). Seroprevalence of SARS-CoV-2 antibodies in people with an acute loss in their sense of smell and/or taste in a community-based population in London, UK: An observational cohort study. PLOS Medicine, 17(10), e1003358. https://doi.org/10.1371/journal.pmed.1003358

Mamtani, M., Athavale, A. M., Abraham, M., Vernik, J., Amarah, A., Ruiz, J., Joshi, A., Itteera, M., Zhukovsky, S., Madaiah, R. P., Hart, P., \& Kulkarni, H. (2020). ASSOCIATION OF HYPERGLYCEMIA WITH HOSPITAL MORTALITY IN COVID-19 PATIENTS WITHOUT DIABETES: A COHORT STUDY. medRxiv, 2020.08.31.20185157. https://doi.org/10.1101/2020.08.31.20185157

Mancilla-Galindo, J., Vera-Zertuche, J. M., Navarro-Cruz, A. R., Segura-Badilla, O., Reyez-Velazquez, G., Tepepa-Lopez, F. J., Aguilar-Alonso, P., Vidal-Mayo, J. de J., \& Kammar-Garcia, A. (2020). Development and Validation of the Patient History COVID-19 (PH-Covid19) Scoring System: A Multivariable Prediction Model of Death in Mexican Patients with COVID-19. medRxiv, 2020.09.05.20189142. https://doi.org/10.1101/2020.09.05.20189142

Mangera, Z., Lewis, A., Hutchinson, J., Searle, L., \& Agrawal, S. (2017). Smoking prevalence in UK hospital admissions from a national observational studyEuropean Respiratory Journal, 50(suppl 61). https://doi.org/10.1183/1393003.congress-2017.PA1268

Mann, C. Z., Abshire, C., Yost, M., Kaatz, S., Swaminathan, L., Flanders, S. A., Prescott, H. C., \& Gagnon-Bartsch, J. A. (2021)Derivation and external validation of a simple risk score to predict inhospital mortality in patients hospitalized for COVID-19 [Preprint]. Emergency Medicine.https://doi.org/10.1101/2021.05.04.21256599 
Manohar, J., Abedian, S., Martini, R., Kulm, S., Salvatore, M., Ho, K., Christos, P., Campion, T., Imperato-McGinley, J., Ibrahim, S., Evering, T. H., Phillips, E., Tamimi, R., Bea, V., D. Balogun, O., Sboner, A., Elemento, O., \& Davis, M. B. (2021). Social and Clinical Determinants of COVID-19 Outcomes: Modeling Real-World Data from a Pandemic Epicenter[Preprint]. Epidemiology. https://doi.org/10.1101/2021.04.06.21254728

Mansour, A., Sajjadi-Jazi, S. M., Kasaeian, A., Khosravi, B., Sorouri, M., Azizi, F., Rajabi, Z., Motamedi, F., Sirusbakht, A., Eslahi, M., Mojtabbavi, H., Sima, A. R., Radmard, A. R., Mohajeri-Tehrani, M. R., \& Abdollahi, M. (2020). Clinical characteristics and outcomes of diabetics hospitalized for COVID-19 infection: A single-centered, retrospective, observational study. EXCLI j, 19, $1533-1543$. https://doi.org/10.17179/excli2020-2988

Maraschini, A., Corsi, E., Salvatore, M. A., \& Donati, S. (2020). Coronavirus and birth in Italy: Results of a national population-based cohort study medRxiv, 2020.06.11.20128652. https://doi.org/10.1101/2020.06.11.20128652

Marcos, M., Belhassen-Garcia, M., Puente, A. S.-., Sampedro-Gomez, J., Azibeiro, R., Dorado-Diaz, P. I., Marcano-Millan, E., Garcia-Vidal, C., Moreiro-Barroso, M. T., Cubino-Boveda, N., PerezGarcia, M. L., Rodriguez-Alonso, B., Encinas-Sanchez, D., Pena-Balbuena, S., Sobejano, E., Diez-Campelo, M., Ines, S., Carbonell, C., Lopez-Parra, M., ... Martin-Oterino, J. A. (2020). Development of a severity of disease score and classification model by machine learning for hospitalized COVID-19 patients. medRxiv, 2020.07.13.20150177. https://doi.org/10.1101/2020.07.13.20150177

Marimuthu, Y., Kunnavil, R., Anil, N., Nagaraja, S. B., Satyanarayana, N., Kumar, J., \& Ramya, B. (2021). Clinical profile and risk factors for mortality among COVID-19 inpatients at a tertiary care centre in Bengaluru, India. Monaldi Archives for Chest Disease. https://doi.org/10.4081/monaldi.2021.1724

Martinez-Lacalzada, M., Adrián Viteri-Noël, L., Manzano, L., Fabregate-Fuente, M., Rubio-Rivas, M., Garcia, S. L., Fernández, F. A., Beato Pérez, J. L., Núñez, J. A. V., Manuel, E. C., Espiño Álvarez, A. C., Castro, S. J. F., Loureiro-Amigo, J., Fontan, P. M. P., Pina, A., María Álvarez Suárez, A., Asiain, A. S., García López, B., Pino, J. L. del, ... for the SEMI-COVID-19 Network. (2020). Predicting critical illness on initial diagnosis of COVID-19 based on easily-obtained clinical variables: Development and validation of the PRIORITY modelPreprint]. Infectious Diseases (except HIV/AIDS). https://doi.org/10.1101/2020.11.27.20237966

Martinez-Lacalzada, M., Viteri-Noël, L. A., Manzano, L., Fabregate-Fuente, M., Rubio-Rivas, M., Garcia, S. L., Fernández, F. A., Pérez, J. L. B., Núñez, J. A. V., Manuel, E. C., Espiño, A.-C., Freire Castro, S. J., Loureiro-Amigo, J., Fontan, P. M. P., Artero, A., Suárez, A. M. Á., Asiain, A. S., López, B. G., Pino, J. L. del, ... for the SEMI-COVID-19 Network. (2020). Predicting critical illness on initial diagnosis of COVID-19: Development and validation of the PRIORITY model for outpatient applicability. https://doi.org/10.1101/2020.11.27.20237966

Martinez-Portilla, R. J., Sotiriadis, A., Torres-Torres, J., Christos, C., Hawkins-Villarreal, A., Villafan-Bernal, J. R., Gurrola-Ochoa, R. A., \& Figueras, F. (2020). Risk factors for mortality in pregnant women with SARS-CoV-2 infection. medRxiv, 2020.05.31.20107276. https://doi.org/10.1101/2020.05.31.20107276

Martinez-Resendez, M. F., Castilleja-Leal, F., Torres-Quintanilla, A., Rojas-Martinez, A., Garcia-Rivas, G., Ortiz-Lopez, R., Trevino, V., Lara-Medrano, R., Villanueva-Lozano, H., Ramirez-Elizondo, T., Sanchez-Nava, V., Moreno-Hoyos, F., Martinez-Thomae, A., Hernandez-Torre, M., Diaz-Olachea, C., Cardona-Huerta, S., Rosa-Pacheco, S. de la, Diaz-Garza, C., Reynoso-Lobo, P., ... TorreAmione, G. (2020). Initial experience in Mexico with convalescent plasma in COVID-19 patients with severe respiratory failure, a retrospective case series. medRxiv, 2020.07.14.20144469. https://doi.org/10.1101/2020.07.14.20144469

Martini, F., D’Alessio, A., Bracchi, F., Di Mauro, D., Fargnoli, A., Motta, M., Giussani, C., Meazza Prina, M., Gobbin, G., \& Taverna, M. (2020). On Cancer, COVID-19, and CT Scans: A Monocentric Retrospective Study. Journal of Clinical Medicine, 9(12), 3935. https://doi.org/10.3390/jcm9123935

Martin-Jimenez, P., Munoz-Garcia, M. I., Seoane, D., Roca-Rodriguez, L., Garcia-Reyne, A., Lalueza, A., Maestro, G., Folgueira, D., Blanco-Palmero, V. A., Martin, A. H.-S., Llamas-Velasco, S., Perez-Martinez, D. A., Gonzalez-Sanchez, M., \& Villarejo-Galende, A. (2020). Cognitive impairment is a common comorbidity in COVID-19 deceased patients. A hospital-based retrospective cohort study. medRxiv, 2020.06.08.20125872. https://doi.org/10.1101/2020.06.08.20125872

Martin-Vicente, M., Almansa, R., Martínez, I., Tedim, A. P., Bustamante, E., Tamayo, L., Aldecoa, C., Gómez, J. M., Renedo, G., Berezo, J. Á., Cedeño, J. A., Mamolar, N., Olivares, P. G., Herrán, R., Cicuendez, R., Enríquez, P., Ortega, A., Jorge, N., Fuente, A. de la, ... Torres, A. (2021). Absent or insufficient anti-SARS-CoV-2 S antibodies at ICU admission are associated to higher viral loads in plasma, antigenemia and mortality in COVID-19 patients [Preprint]. Infectious Diseases (except HIV/AIDS).https://doi.org/10.1101/2021.03.08.21253121

Matli, K., Chamoun, N., Fares, A., Zibara, V., Osta, S. A., Nasrallah, R., Salameh, P., Mokhbat, J., \& Ghanem, G. (2021).Combined Anticoagulant and antiplatelet therapy is associated with an improved outcome in hospitalized COVID-19 patients: A propensity matched cohort study [Preprint]. Cardiovascular Medicine. https://doi.org/10.1101/2021.07.13.21260467

Maucourant, C., Filipovic, I., Ponzetta, A., Aleman, S., Cornillet, M., Hertwig, L., Strunz, B., Lentini, A., Reinius, B., Brownlie, D., Gomez, A. C., Ask, E. H., Hull, R. M., Haroun-Izquierdo, A., Schaffer, M., Klingstrom, J., Folkesson, E., Buggert, M., Sandberg, J. K., ... Group, K. C. S. (2020). Natural killer cell activation related to clinical outcome of COVID-19. medRxiv, 2020.07.07.20148478. https://doi.org/10.1101/2020.07.07.20148478

Márquez-Salinas, A., Fermín-Martínez, C. A., Antonio-Villa, N. E., Vargas-Vázquez, A., Guerra, E. C., Campos-Muñoz, A., Zavala-Romero, L., Mehta, R., Bahena-López, J. P., Ortiz-Brizuela, E. González-Lara, M. F., Roman-Montes, C. M., Martinez-Guerra, B. A., Leon, A. P. de, Sifuentes-Osornio, J., Gutiérrez-Robledo, L. M., Aguilar-Salinas, C. A., \& Bello-Chavolla, O. Y. (2020). Adaptive metabolic and inflammatory responses identified using accelerated aging metrics are linked to adverse outcomes in severe SARS-CoV-2 infection [Preprint]. Geriatric Medicine. https://doi.org/10.1101/2020.11.03.20225375

McGrail, D. E., \& Edwards, D. (2020). COVID-19 Case Series at UnityPoint Health St. Lukes Hospital in Cedar Rapids, IAmedRxiv, 2020.07.17.20156521. https://doi.org/10.1101/2020.07.17.20156521

McQueenie, R., Foster, H., Jani, B. D., Katikireddi, S. V., Sattar, N., Pell, J. P., Ho, F. K., Niedzwiedz, C. L., Hastie, C. E., Anderson, J., Mark, P. B., Sullivan, M., ODonnell, C. A., Mair, F. S., \& Nicholl, B. I. (2020). Multimorbidity, Polypharmacy, and COVID-19 infection within the UK Biobank cohort. medRxiv, 2020.06.10.20127563. https://doi.org/10.1101/2020.06.10.20127563

Meini, S., Fortini, A., Andreini, R., Sechi, L. A., \& Tascini, C. (n.d.). The Paradox of the Low Prevalence of Current Smokers Among Covid-19 Patients Hospitalized in Non-Intensive Care Wards: Results From an Italian Multicenter Case-Control Study. Nicotine \& Tobacco Research. https://doi.org/10.1093/ntr/ntaa188 
Mejia-Vilet, J. M., Cordova-Sanchez, B. M., Fernandez-Camargo, D., Mendez-Perez, R. A., Morales-Buenrostro, L. E., \& Hernandez-Gilsoul, T. (2020). A Risk Score to Predict Admission to Intensive Care Unit in Patients With COVID-19: The ABC-GOALS Score. medRxiv, 2020.05.12.20099416. https://doi.org/10.1101/2020.05.12.20099416

Melo, A. C. de, Thuler, L. C. S., Silva, J. L. da, Albuquerque, L. Z. de, Pecego, A. C., Rodrigues, L. O. R., Conceicao, M. S. da, Garrido, M. M., Mendes, G. L., Pereira, A. C. M., Soares, M. A., Viola, J. P. B., \& Force, I. C. T. (2020). Cancer inpatient with COVID-19: A report from the Brazilian National Cancer Institute. medRxiv, 2020.06.27.20141499. https://doi.org/10.1101/2020.06.27.20141499 Melotti, R., Scaggiante, F., Falciani, M., Weichenberger, C. X., Foco, L., Lombardo, S., Grandi, A. D., Laer, D. von, Mahlknecht, A., Pramstaller, P. P., Pagani, E., Meier, H., Gaertner, T., Troi, C., Mascalzoni, D., Pattaro, C., \& Mian, M. (2021). Prevalence and determinants of serum antibodies to SARS-CoV-2 in the general population of the Gardena ValleyPreprint]. Public; Global Health. https://doi.org/10.1101/2021.03.19.21253883

Mendes, A., Herrmann, F. R., Genton, L., Serratrice, C., Carrera, E., Vargas, M. I., Gold, G., Graf, C. E., Zekry, D., \& Scheffler, M. (2021). Incidence, characteristics and clinical relevance of acute stroke in old patients hospitalized with COVID-19. BMC Geriatr, 21. https://doi.org/10.1186/s12877-021-02006-2

Mendez-Dominguez, N., Santos-Zaldívar, K., Gomez-Carro, S., Datta-Banik, S., \& Carrillo, G. (2021). Maternal mortality during the COVID-19 pandemic in Mexico: A preliminary analysis during the first year. BMC Public Health, 21(1), 1297. https://doi.org/10.1186/s12889-021-11325-3

Mendy, A., Apewokin, S., Wells, A. A., \& Morrow, A. L. (2020). Factors Associated with Hospitalization and Disease Severity in a Racially and Ethnically Diverse Population of COVID-19 Patients. medRxiv, 2020.06.25.20137323. https://doi.org/10.1101/2020.06.25.20137323

Menges, D., Ballouz, T., Anagnostopoulos, A., Aschmann, H. E., Domenghino, A., Fehr, J. S., \& Puhan, M. A. (2021).Burden of Post-COVID-19 Syndrome and Implications for Healthcare Service Planning: A Population-based Cohort Study [Preprint]. Epidemiology. https://doi.org/10.1101/2021.02.27.21252572

Merkely, B., Szabó, A. J., Kosztin, A., Berényi, E., Sebestyén, A., Lengyel, C., Merkely, G., Karády, J., Várkonyi, I., Papp, C., Miseta, A., Betlehem, J., Burián, K., Csóka, I., Vásárhelyi, B., Ludwig, E., Prinz, G., Sinkó, J., Hankó, B., ... for the HUNgarian COronaVirus-19 Epidemiological Research (H-UNCOVER) investigators. (2020). Novel coronavirus epidemic in the Hungarian population, a cross-sectional nationwide survey to support the exit policy in Hungary. GeroScience. https://doi.org/10.1007/s11357-020-00226-9

Merzon, E., Tworowski, D., Gorohovski, A., Vinker, S., Cohen, A. G., Green, I., \& Morgenstern, M. F. (2020). Low plasma 25(OH) vitamin D3 level is associated with increased risk of COVID-19 infection: An Israeli population-based study. medRxiv, 2020.07.01.20144329. https://doi.org/10.1101/2020.07.01.20144329

Miyara, M., Tubach, F., Martinez, V., Morelot-Panzini, C., Pernet, J., Haroche, J., Lebbah, S., Morawiec, E., Gorochov, G., Caumes, E., Hausfater, P., Combes, A., Similowski, T., \& Amoura, Z. (2020). Low rate of daily smokers in patients with symptomatic COVID-19. medRxiv, 2020.06.10.20127514. https://doi.org/10.1101/2020.06.10.20127514

Mo, P., Xing, Y., Xiao, Y., Deng, L., Zhao, Q., Wang, H., Xiong, Y., Cheng, Z., Gao, S., Liang, K., Luo, M., Chen, T., Song, S., Ma, Z., Chen, X., Zheng, R., Cao, Q., Wang, F., \& Zhang, Y. (n.d.) Clinical characteristics of refractory COVID-19 pneumonia in Wuhan, China. Clinical Infectious Diseases. https://doi.org/10.1093/cid/ciaa270

Modrák, M., Bürkner, P.-C., Sieger, T., Slisz, T., Vašáková, M., Mesežnikov, G., Casas-Mendez, L. F., Vajter, J., Táborský, J., Kubricht, V., Suk, D., Horejsek, J., Jedlička, M., Mifková, A., Jaroš, A., Kubiska, M., Váchalová, J., Šín, R., Veverková, M., ... Hyánek, T. (2020). Detailed disease progression of 213 patients hospitalized with Covid-19 in the Czech Republic: An exploratory analysis https://doi.org/10.1101/2020.12.03.20239863

Mohamed-Hussein, A., Galal, I., Saad, M., Zayan, H. E., Abdelsayed, M., Moustafa, M., Ezzat, A. R., Helmy, R., Elaal, H. A., Aly, K., \& Abderheem, S. (2020). Post-COVID-19 Functional Status: Relation to age, smoking, hospitalization and comorbidities. medRxiv, 2020.08.26.20182618. https://doi.org/10.1101/2020.08.26.20182618

Mohamud, A. Y., Griffith, B., Rehman, M., Miller, D., Chebl, A., Patel, S. C., Howell, B., Kole, M., \& Marin, H. (2020). Intraluminal Carotid Artery Thrombus in COVID-19: Another Danger of Cytokine Storm? American Journal of Neuroradiology. https://doi.org/10.3174/ajnr.A6674

Mok, J., Malpartida, J. C., O'Dell, K., Davis, J., Gao, C., \& Manyam, H. (2021). Vascular comorbidities worsen prognosis of patients with heart failure hospitalised with COVID-190pen Heart, 8(1), e001668. https://doi.org/10.1136/openhrt-2021-001668

Molenaar, N. M., Rommel, A.-S., Witte, L. de, Dolan, S. M., Lieb, W., Ibroci, E., Ohrn, S., Lynch, J., Capuano, C., Stadlbauer, D., Krammer, F., Zapata, L. B., Brody, R. I., Sperling, R. S., Afzal, O., Missall, M. R., Balbierz, A., Janevic, T., Stone, J., ... Bergink, V. (2021). Seroprevalence of SARS-CoV-2 during pregnancy and associated outcomes: Results from an ongoing prospective cohort study, New York City. https://doi.org/10.1101/2021.02.01.21250943

Mollan, K. R., Eron, J. J., Krajewski, T. J., Painter, W., Duke, E. R., Morse, C. G., Goecker, E. A., Premkumar, L., Wolfe, C. R., Szewczyk, L. J., Alabanza, P. L., Loftis, A. J., Degli-Angeli, E. J., Brown, A. J., Dragavon, J. A., Won, J. J., Keys, J., Hudgens, M. G., Fang, L., ... Fischer, W. A. (2021). Infectious SARS-CoV-2 Virus in Symptomatic COVID-19 Outpatients: Host, Disease, and Viral Correlates [Preprint]. Infectious Diseases (except HIV/AIDS).https://doi.org/10.1101/2021.05.28.21258011

Monteiro, A. C. C., Suri, R., Emeruwa, I. O., Stretch, R. J., Lopez, R. Y. C., Sherman, A., Lindsay, C. C., Fulcher, J. A., Goodman-Meza, D., Sapru, A., Buhr, R. G., Chang, S. Y., Wang, T., \& Qadir, N. (2020). Obesity and Smoking as Risk Factors for Invasive Mechanical Ventilation in COVID-19: A Retrospective, Observational Cohort Study. medRxiv, 2020.08.12.20173849. https://doi.org/10.1101/2020.08.12.20173849

Mora, A. M., Lewnard, J. A., Kogut, K., Rauch, S. A., Morga, N., Hernandez, S., Wong, M. P., Huen, K., Chang, C., Jewell, N. P., Holland, N., Harris, E., Cuevas, M., \& Eskenazi, B. (2021)Risk factors for SARS-CoV-2 infection among farmworkers in Monterey County, California. https://doi.org/10.1101/2021.02.01.21250963

Morshed, M. S., Mosabbir, A. A., Chowdhury, P., Ashadullah, S. M., \& Hossain, M. S. (2020). Clinical manifestations of patients with Coronavirus Disease 2019 (COVID- 19 ) attending at hospitals in Bangladesh. medRxiv, 2020.07.30.20165100. https://doi.org/10.1101/2020.07.30.20165100

Mostafa, A., El-Sayed, M. H., El-Meteini, M., Saleh, A., Omar, A., Mansour, O., Girgis, S., Hafez, H., \& Kandil, S. (2021). SARS-Co-V2 infection in never, former, and current tobacco/nicotine users: A cohort study of 4040 Egyptian healthcare workers. BMC Public Health, 21(1), 1243. https://doi.org/10.1186/s12889-021-11290-x

Motta, J. K., Ogunnaike, R. O., Shah, R., Stroever, S., Cedeno, H. V., Thapa, S. K., Chronakos, J. J., Jimenez, E. J., Petrini, J., \& Hegde, A. (2020). Clinical Outcomes With the Use of Prophylactic 
Versus Therapeutic Anticoagulation in COVID-19. medRxiv, 2020.07.20.20147769. https://doi.org/10.1101/2020.07.20.20147769

Muñoz, F. J. T., García-Guijarro, E., García-Domingo, P., Pérez-Nieto, G., Rojas, F. R., García-Peña, M., Gallo, M. A. N., Bermejo, J. A. M., García-Monge, M. T. de G., \& Granizo, J. J. (2020). A safe protocol to identify low risk patients with COVID-19 pneumonia for outpatient management. medRxiv, 2020.12.15.20229286. https://doi.org/10.1101/2020.12.15.20229286

Murray, E. (2020). Causation in smoking and COVID-19. https://twitter.com/EpiEllie/status/1258607277357006849?s=20

Mutambudzi, M., Niedzwiedz, C. L., Macdonald, E. B., Leyland, A. H., Mair, F. S., Anderson, J. J., Celis-Morales, C. A., Cleland, J., Forbes, J., Gill, J. M., Hastie, C., Ho, F. K., Jani, B. D., Mackay, D. F., Nicholl, B. I., O'Donnell, C. A., Sattar, N. I., Welsh, P. I., Pell, J. P., ... Demou, E. (2020). Occupation and risk of severe COVID-19: Prospective cohort study of 120,075 UK Biobank participants. medRxiv, 2020.05.22.20109892. https://doi.org/10.1101/2020.05.22.20109892

Nanda, S., Chacin Suarez, A. S., Toussaint, L., Vincent, A., Fischer, K. M., Hurt, R., Schroeder, D. R., Medina Inojosa, J. R., O’Horo, J. C., DeJesus, R. S., Abu Lebdeh, H. S., Mundi, M. S., Iftikhar, S., \& Croghan, I. T. (2021). Body Mass Index, Multi-Morbidity, and COVID-19 Risk Factors as Predictors of Severe COVID-19 Outcomes. Journal of Primary Care \& Community Health 12, 215013272110185. https://doi.org/10.1177/21501327211018559

Nassar, Y., Mokhtar, A., Elhadidy, A., Elsayed, M., Mostafa, F., Rady, A., Eladawy, A., Elshazly, M., Saeed, M., Mokhtar, S., Buschbeck, S., \& Sakr, Y. (2021). Outcomes and risk factors for death in patients with coronavirus disease-2019 (COVID-19) pneumonia admitted to the intensive care units of an Egyptian University Hospital. A retrospective cohort study. Journal of Infection and Public Health, S1876034121001787. https://doi.org/10.1016/j.jiph.2021.06.012

Nezhadmoghadam, F., \& Tamez-Peña, J. (2021). Unsupervised Discovery of Risk Profiles on Negative and Positive COVID-19 Hospitalized Patients https://doi.org/10.1101/2020.12.30.20248908

Nguyen, A. B., Upadhyay, G. A., Chung, B., Smith, B., Besser, S. A., Johnson, J. A., Blair, J., Ward, R. P., DeCara, J., Polonsky, T., Patel, A. R., Grinstein, J., Holzhauser, L., Kalathiya, R., Shah, A. P., Paul, J., Nathan, S., Liao, J., Lang, R. M., ... Tung, R. (2020). Outcomes and Cardiovascular Comorbidities in a Predominantly African-American Population with COVID-19. medRxiv, 2020.06.28.20141929. https://doi.org/10.1101/2020.06.28.20141929

Nicholson, C. J., Wooster, L., Sigurslid, H. H., Li, R. F., Jiang, W., Tian, W., Cardenas, C. L., \& Malhotra, R. (2020). Estimating Risk of Mechanical Ventilation and Mortality Among Adult CoVID-19 patients Admitted to Mass General Brigham: The VICE and DICE Scores. medRxiv, 2020.09.14.20194670. https://doi.org/10.1101/2020.09.14.20194670

Niedzwiedz, C. L., O’Donnell, C. A., Jani, B. D., Demou, E., Ho, F. K., Celis-Morales, C., Nicholl, B. I., Mair, F. S., Welsh, P., Sattar, N., Pell, J. P., \& Katikireddi, S. V. (2020). Ethnic and socioeconomic differences in SARS-CoV-2 infection: Prospective cohort study using UK Biobank. BMC Medicine, 18(1), 160. https://doi.org/10.1186/s12916-020-01640-8

Nielsen, K. J., Vestergaard, J. M., Schlünssen, V., Bonde, J. P., Kaspersen, K. A., Biering, K., Carstensen, O., Greve, T., Hansen, K. K., Dalbøge, A., Flachs, E. M., Jespersen, S., Hansen, M. L., Mikkelsen, S., Thomsen, M. K., Redder, J. D., Würtz, E. T., Østergaard, L., Erikstrup, C., \& Kolstad, H. A. (2021). Day-by-day symptoms following positive and negative PCR tests for SARS-CoV-2 in non-hospitalized healthcare workers: A 90-day follow-up study. International Journal of Infectious Diseases, 108, 382-390. hittps://doi.org/10.1016/i.iiid.2021.05.032

Noh, C. S., Kim, W.-Y., \& Baek, M. S. (2021). Risk factors associated with the need for oxygen therapy in patients with COVID-19Medicine, 100(18), e25819. https://doi.org/10.1097/MD.0000000000025819

Nunez-Gil, I. J. J., Fernandez-Ortiz, A., Maroud Eid, C., Huang, J., Romero, R., Becerra-Munoz, V. M., Uribarri, A., Feltes, G., Trabatoni, D., Fernandez-Rozas, I., Viana-Llamas, M. C., Pepe, M., Cerrato, E., Bertaina, M., Capel Astrua, T., Alfonso, E., Castro-Mejia, A. F., Raposeiras-Roubin, S., D’Ascenzo, F., ... Macaya, C. (2020). Underlying heart diseases and acute COVID-19 outcomes. Cardiol J, NA. https://doi.org/10.5603/CJ.a2020.0183

Nuño, M., García, Y., Rajasekar, G., Pinheiro, D., \& Schmidt, A. J. (2021).COVID-19 Hospitalizations in Five California Hospitals. https://doi.org/10.1101/2021.01.29.21250788

Núñez-Gil, I. J., Fernández-Ortiz, A., Maroud Eid, C., Huang, J., Romero, R., Becerra-Muñoz, V. M., Uribarri, A., Feltes, G., Trabatoni, D., Fernandez-Rozas, I., Viana-Llamas, M. C., Pepe, M., Cerrato, E., Bertaina, M., Capel Astrua, T., Alfonso, E., Castro-Mejía, A. F., Raposeiras-Roubin, S., D'Ascenzo, F., ... Macaya, C. (2021). Underlying heart diseases and acute COVID-19 outcomes. Cardiology Journal, 28(2), 202-214. https://doi.org/10.5603/CJ.a2020.0183

O'Gallagher, K., Shek, A., Bean, D. M., Bendayan, R., Teo, J. T. H., Dobson, R. J. B., Shah, A. M., \& Zakeri, R. (2020)Pre-existing cardiovascular disease rather than cardiovascular risk factors drives mortality in COVID-19 [Preprint]. Cardiovascular Medicine. https://doi.org/10.1101/2020.12.02.20242933

O'Reilly, G. M., Mitchell, R. D., Mitra, B., Akhlaghi, H., Tran, V., Furyk, J. S., Buntine, P., Bannon-Murphy, H., Amos, T., Udaya Kumar, M., Perkins, E., Prentice, A., Szwarcberg, O., Loughman, A., Lowry, N., Colwell, S., Noonan, M. P., Hiller, R., Paton, A., ... the COVED Project Team. (2020). Epidemiology and clinical features of emergency department patients with suspected and confirmed <span style="font-variant:small-caps;">COVID</span> -19: A multisite report from the <span style="font-variant:small-caps;">COVID-19 Emergency Department</span> Quality Improvement Project for July 2020 ( <span style="font-variant:small-caps;">COVED</span> -3). Emergency Medicine Australasia, 1742-6723.13651. https://doi.org/10.1111/1742-6723.13651

Oakes, J. M., Fuchs, R. M., Gardner, J. D., Lazartigues, E., \& Yue, X. (2018). Nicotine and the renin-angiotensin systemAm. J. Physiol. - Regul. Integr. Comp. Physiol 315, 895-906. https://doi.org/10.1152/ajpregu.00099.2018

Odani, S. (2018). Tobacco Product Use Among Military Veterans — United States, 2010-2015.MMWR. Morbidity and Mortality Weekly Report 67. https://doi.org/10.15585/mmwr.mm6701a2

Olivares, F., Munoz, D., Fica, A., Delama, I., Alvarez, I., Navarrete, M., Blackburn, E., Garrido, P., \& Granjean, J. (2020). Covid-19 in Chile. The experience of a Regional reference Center. Preliminary report. medRxiv, 2020.06.14.20130898. https://doi.org/10.1101/2020.06.14.20130898

Oliveira, E., Parikh, A., Lopez-Ruiz, A., Carrillo, M., Goldberg, J., Cearras, M., Fernainy, K., Andersen, S., Mercado, L., Guan, J., Zafar, H., Louzon, P., Carr, A., Baloch, N., Pratley, R., Silvestry, S., Hsu, V., Sniffen, J., Herrera, V., \& Finkler, N. (2020). ICU Outcomes and Survival in Patients with Severe COVID-19 in the Largest Health Care System in Central Florida. medRxiv, 2020.08.25.20181909. https://doi.org/10.1101/2020.08.25.20181909

Omrani, A. S., Almaslamani, M. A., Daghfal, J., Alattar, R. A., Elgara, M., Shaar, S. H., Ibrahim, T., Zaqout, A., Bakdach, D., Akkari, A., Baiou, A., Alhariri, B., Elajez, R., Husain, A., Badawi, M. N., Abid, F. B., Jarir, S. A., Abdalla, S., Kaleeckal, A., ... Kuwari, H. M. A. (2020). The First Consecutive 5000 Patients with Coronavirus Disease 2019 from Qatar; a Nation-wide Cohort Study. medRxiv, 
2020.07.15.20154690. https://doi.org/10.1101/2020.07.15.20154690

Organisation, W. H. (n.d.). Laboratory testing for 2019 novel coronavirus (2019-nCoV) in suspected human cases https://www.who.int/publications-detail-redirect/10665-331501

Ouyang, J., Shan, X., Wang, X., Zhang, X., Chen, Y., Qi, M., Xia, C., Gu, D., Chen, Y., \& Zhang, B. (2020). Clinical characteristics of COVID-19 and the model for predicting the occurrence of critically ill patients: A retrospective cohort study. medRxiv, 2020.08.13.20173799. https://doi.org/10.1101/2020.08.13.20173799

Palaiodimos, L., Kokkinidis, D. G., Li, W., Karamanis, D., Ognibene, J., Arora, S., Southern, W. N., \& Mantzoros, C. S. (2020). Severe obesity, increasing age and male sex are independently associated with worse in-hospital outcomes, and higher in-hospital mortality, in a cohort of patients with COVID-19 in the Bronx, New York. Metabolism, $108,154262$. https://doi.org/10.1016/j.metabol.2020.154262

Paleiron, N., Mayet, A., Marbac, V., Perisse, A., Barazzutti, H., Brocq, F.-X., Janvier, F., Bertrand, D., \& Bylicki, O. (2021). Impact of Tobacco Smoking on the risk of COVID-19.A large scale retrospective cohort study. Nicotine Tob Res, NA. https://doi.org/10.1093/ntr/ntab004

Pan, A., Khan, O., Meeks, J., Boom, M., Masud, F., Andrieni, J., Phillips, R., Tiruneh, Y., Kash, B., \& Vahidy, F. (2020). Disparities in COVID-19 Hospitalizations and Mortality among Black and Hispanic Patients: Cross-Sectional Analysis from the Greater Houston Metropolitan Area. medRxiv, 2020.08.19.20177956. https://doi.org/10.1101/2020.08.19.20177956

Pandolfi, L., Fossali, T., Frangipane, V., Bozzini, S., Morosini, M., D’Amato, M., Lettieri, S., Urtis, M., Toro, A. D., Saracino, L., Percivalle, E., Tomaselli, S., Cavagna, L., Cova, E., Mojoli, F., Bergomi, P., Ottolina, D., Lilleri, D., Corsico, A. G., ... Meloni, F. (2020). Broncho-alveolar inflammation in COVID-19 patients: A correlation with clinical outcome. medRxiv, 2020.07.17.20155978. https://doi.org/10.1101/2020.07.17.20155978

Park, B. E., Lee, J. H., Park, H. K., Kim, H. N., Jang, S. Y., Bae, M. H., Yang, D. H., Park, H. S., Cho, Y., Lee, B. Y., Nam, C. W., Lee, J. B., Kim, U., Chae, S. C., \& Daegu COVID-19 Research Project. (2021). Impact of Cardiovascular Risk Factors and Cardiovascular Diseases on Outcomes in Patients Hospitalized with COVID-19 in Daegu Metropolitan City. J Korean Med Sci, 36. https://doi.org/10.3346/jkms.2021.36.e15

Parra-Bracamonte, G. M., Lopez-Villalobos, N., \& Parra-Bracamonte, F. E. (2020). Clinical characteristics and risk factors for mortality of patients with COVID-19 in a large data set from Mexico. Annals of Epidemiology, 52, 93-98.e2. https://doi.org/10.1016/j.annepidem.2020.08.005

Parrotta, E., Kister, I., Charvet, L., Sammarco, C., Saha, V., Charlson, R. E., Howard, J., Gutman, J. M., Gottesman, M., Abou-Fayssal, N., Wolintz, R., Keilson, M., Fernandez-Carbonell, C., Krupp, L. B., \& Zhovtis Ryerson, L. (2020). COVID-19 outcomes in MS: Observational study of early experience from NYU Multiple Sclerosis Comprehensive Care Center. Neurology - Neuroimmunology Neuroinflammation, 7(5), e835. https://doi.org/10.1212/NXI.0000000000000835

Patanavanich, R., \& Glantz, S. A. (2020). Smoking is Associated with COVID-19 Progression: A Meta-Analysis.medRxiv. https://doi.org/10.14171/j.2095-5944.sg.2014.02.004

Patel, M., Chowdhury, J., Mills, N., Marron, R., Gangemi, A., Dorey-Stein, Z., Yousef, I., Zheng, M., Tragesser, L., Giurintano, J., Gupta, R., Rali, P., D’Alonzo, G., Zhao, H., Patlakh, N., Marchetti, N., Criner, G., \& Gordon, M. (2020). ROX Index Predicts Intubation in Patients with COVID-19 Pneumonia and Moderate to Severe Hypoxemic Respiratory Failure Receiving High Flow Nasal Therapy. medRxiv, 2020.06.30.20143867. https://doi.org/10.1101/2020.06.30.20143867

Patone, M., Thomas, K., Hatch, R., Tan, P. S., Coupland, C., Liao, W., Mouncey, P., Harrison, D., Rowan, K., Horby, P., Watkinson, P., \& Hippisley-Cox, J. (2021). Mortality and critical care unit admission associated with the SARS-CoV-2 lineage B.1.1.7 in England: An observational cohort study. The Lancet Infectious Diseases, S1473309921003182. https://doi.org/10.1016/S1473$\underline{3099(21) 00318-2}$

Peng, F., Lei, S., Zhang, Q., Zhong, Y., \& Wu, S. (2021). Smoking Is Correlated With the Prognosis of Coronavirus Disease 2019 (COVID-19) Patients: An Observational StudyFrontiers in Physiology, 12, 634842. https://doi.org/10.3389/fphys.2021.634842

Perico, L., Tomasoni, S., Peracchi, T., Perna, A., Pezzotta, A., Remuzzi, G., \& Benigni, A. (2020). COVID-19 and lombardy: TESTing the impact of the first wave of the pandemicEBioMedicine, 61. https://doi.org/10.1016/j.ebiom.2020.103069

Perrone, F., Piccirillo, M. C., Ascierto, P. A., Salvarani, C., Parrella, R., Marata, A. M., Popoli, P., Ferraris, L., Trischitta, M. M. M., Ripamonti, D., Binda, F., Bonfanti, P., Squillace, N., Castelli, F., Muiesan, M. L., Lichtner, M., Calzetti, C., Salerno, N. D., Atripaldi, L., ... Gallo, C. (2020). Tocilizumab for patients with COVID-19 pneumonia. The TOCIVID-19 prospective phase 2 trial. medRxiv, 2020.06.01.20119149. https://doi.org/10.1101/2020.06.01.20119149

Peters, E. J., Collard, D., Assen, S. van, Beudel, M., Bomers, M. K., Buijs, J., Haan, L. de, Ruijter, W. de, Douma, R., Elbers, P. W., Goorhuis, A., Oever, N. C. G. van den, Knarren, G. H., Moeniralam, H. S., Mostard, R. L., Quanjel, M. J., Reidinga, A. C., Renckens, R., Bergh, J. P. van den, ... Sikkens, J. J. (2020). Outcomes of Persons With COVID-19 in Hospitals With and Without Standard Treatment With (Hydroxy)chloroquine. medRxiv, 2020.08.14.20173369. https://doi.org/10.1101/2020.08.14.20173369

Petrilli, C. M., Jones, S. A., Yang, J., Rajagopalan, H., O’Donnell, L., Chernyak, Y., Tobin, K. A., Cerfolio, R. J., Francois, F., \& Horwitz, L. I. (2020). Factors associated with hospital admission and critical illness among 5279 people with coronavirus disease 2019 in New York City: Prospective cohort study. BMJ, 369. https://doi.org/10.1136/bmi.m1966

Philipose, Z., Smati, N., Wong, C. S. J., Aspey, K., \& Mendall, M. A. (2020). Obesity, old age and frailty are the true risk factors for COVID-19 mortality and not chronic disease or ethnicity in Croydon. medRxiv, 2020.08.12.20156257. https://doi.org/10.1101/2020.08.12.20156257

PHOSP-COVID Collaborative Group, Evans, R. A., McAuley, H., Harrison, E. M., Shikotra, A., Singapuri, A., Sereno, M., Elneima, O., Docherty, A. B., Lone, N. I., Leavy, O. C., Daines, L., Baillie, J. K., Brown, J. S., Chalder, T., De Soyza, A., Diar Bakerly, N., Easom, N., Geddes, J. R., ... Brightling, C. E. (2021). Physical, cognitive and mental health impacts of CoVID-19 following hospitalisation - a multi-centre prospective cohort study [Preprint]. Infectious Diseases (except HIV/AIDS).https://doi.org/10.1101/2021.03.22.21254057

Polubriaginof, F., Salmasian, H., Albert, D. A., \& Vawdrey, D. K. (2018). Challenges with Collecting Smoking Status in Electronic Health RecordsAMIA Annual Symposium Proceedings, 2017, 13921400. https://www.ncbi.nIm.nih.gov/pmc/articles/PMC5977725/

Pongpirul, W. A., Wiboonchutikul, S., Charoenpong, L., Panitantum, N., Vachiraphan, A., Uttayamakul, S., Pongpirul, K., Manosuthi, W., \& Prasithsirikul, W. (2020). Clinical course and potential 
predicting factors of pneumonia of adult patients with coronavirus disease 2019 (COVID-19): A retrospective observational analysis of 193 confirmed cases in Thailand. medRxiv, 2020.06.24.20139642. https://doi.org/10.1101/2020.06.24.20139642

Prats-Uribe, A., Tobed, M., Villacampa, J. M., Agüero, A., García-Bastida, C., Tato, J. I., Rodrigáñez, L., Holguera, V. D., Hernández-García, E., Poletti, D., Simonetti, G., Villarraga, V., MelerClaramonte, C., Barrueco, Á. S., Chiesa-Estomba, C., Casasayas, M., Parente-Arias, P., Castro, P., Prieto-Alhambra, D., ... TraqueoCOVID SEORL Group. (2021). Timing of elective tracheotomy and duration of mechanical ventilation amongst patients admitted to intensive care with severe COVID-19: A multicentre prospective cohort study. https://doi.org/10.1101/2021.01.22.21249651

Prats-Uribe, A., Xie, J., Prieto-Alhambra, D., \& Petersen, I. (2021). Smoking and COVID-19 Infection and Related Mortality: A Prospective Cohort Analysis of UK Biobank Data.Clinical Epidemiology, Volume 13, 357-365. https://doi.org/10.2147/CLEP.S300597

Printza, A., Katotomichelakis, M., Valsamidis, K., Metallidis, S., Panagopoulos, P., Panopoulou, M., Petrakis, V., \& Constantinidis, J. (2021). Smell and Taste Loss Recovery Time in COVID-19 Patients and Disease Severity. Journal of Clinical Medicine, 10(5), 966. https://doi.org/10.3390/jcm10050966

Pritchard, M., Dankwa, E. A., Hall, M., Baillie, J. K., Carson, G., Docherty, A., Donnelly, C. A., Dunning, J., Fraser, C., Hardwick, H., Harrison, E. M., Holden, K. A., Kartsonaki, C., Kennon, K., Lee, J., McLean, K., Openshaw, P. J. M., Plotkin, D., Rojek, A., ... Partners, on behalf of the I. C. (2020). ISARIC Clinical Data Report 4 October 2020. medRxiv, 2020.07.17.20155218. https://doi.org/10.1101/2020.07.17.20155218

Puebla Neira, D., Watts, A., Seashore, J., Polychronopoulou, E., Kuo, Y.-F., \& Sharma, G. (2021). Smoking and risk of COVID-19 hospitalizationRespiratory Medicine, $182,106414$. https://doi.org/10.1016/j.rmed.2021.106414

Qi, D., Yan, X., Tang, X., Peng, J., Yu, Q., Feng, L., Yuan, G., Zhang, A., Chen, Y., Yuan, J., Huang, X., Zhang, X., Hu, P., Song, Y., Qian, C., Sun, Q., Wang, D., Tong, J., \& Xiang, J. (2020). Epidemiological and clinical features of 2019-nCoV acute respiratory disease cases in Chongqing municipality, China: A retrospective, descriptive, multiple-center study. medRxiv, 2020.03.01.20029397. https://doi.org/10.1101/2020.03.01.20029397

Qu, J., Chang, L. K., Tang, X., Du, Y., Yang, X., Liu, X., Han, P., \& Xue, Y. (2020). Clinical characteristics of COVID-19 and its comparison with influenza pneumonia Acta Clinica Belgica, O(0), 1-9. https://doi.org/10.1080/17843286.2020.1798668

Quan, D., Luna Wong, L., Shallal, A., Madan, R., Hamdan, A., Ahdi, H., Daneshvar, A., Mahajan, M., Nasereldin, M., Van Harn, M., Opara, I. N., \& Zervos, M. (2021). Impact of Race and Socioeconomic Status on Outcomes in Patients Hospitalized with COVID-19. J Gen Intern Med, NA. https://doi.org/10.1007/s11606-020-06527-1

Qureshi, A. I., Baskett, W. I., Huang, W., Myers, D., Lobanova, I., Ishfaq, M. F., Naqvi, S. H., French, B. R., Chandrasekaran, P. N., Siddiq, F., Gomez, C. R., \& Shyu, C.-R. (2021). Intracerebral Hemorrhage and Coronavirus Disease 2019 in a Cohort of 282,718 Hospitalized Patients. Neurocritical Care. https://doi.org/10.1007/s12028-021-01297-y

Rachmawati, E., Listiowati, E., Kurniawan, D. W., Suraya, I., Ahsan, A., \& Nurmansyah, M. I. (2021a). Significance of Chronic Diseases and Smoking Behavior in the Development of Acute Respiratory Distress Syndrome Among Hospitalized COVID-19 Patients in Indonesia. Asia Pacific Journal of Public Health 33(4), 427-430. https://doi.org/10.1177/10105395211002624

Rachmawati, E., Listiowati, E., Kurniawan, D. W., Suraya, I., Ahsan, A., \& Nurmansyah, M. I. (2021b). Significance of Chronic Diseases and Smoking Behavior in the Development of Acute Respiratory Distress Syndrome Among Hospitalized COVID-19 Patients in Indonesia. Asia Pacific Journal of Public Health 33(4), 427-430. https://doi.org/10.1177/10105395211002624

Radon, K., Bakuli, A., Pütz, P., Gleut, R. L., Guggenbuehl Noller, J. M., Olbrich, L., Saathoff, E., Garí, M., Schälte, Y., Frahnow, T., Wölfel, R., Pritsch, M., Rothe, C., Pletschette, M., Rubio-Acero, R., Beyerl, J., Metaxa, D., Forster, F., Thiel, V., ... Fuchs, C. (2021). From first to second wave: Follow-up of the prospective Covid-19 cohort (KoCo19) in Munich (Germany[Preprint]. Epidemiology https://doi.org/10.1101/2021.04.27.21256133

Raines, A. M., Tock, J. L., McGrew, S. J., Ennis, C. R., Derania, J., Jardak, C. L., Lim, J. H., Boffa, J. W., Houtsma, C., Jones, K. R., Martin-Klinger, C., Widmer, K., Schapira, R., Zvolensky, M. J., Hoerger, M., Constans, J. I., \& Franklin, C. L. (2021). Correlates of death among SARS-CoV-2 positive veterans: The contribution of lifetime tobacco use. Addictive Behaviors, $113,106692$. https://doi.org/10.1016/j.addbeh.2020.106692

Raisi-Estabragh, Z., McCracken, C., Bethell, M. S., Cooper, J., Cooper, C., Caulfield, M. J., Munroe, P. B., Harvey, N. C., \& Petersen, S. E. (n.d.). Greater risk of severe COVID-19 in Black, Asian and Minority Ethnic populations is not explained by cardiometabolic, socioeconomic or behavioural factors, or by 25(OH)-vitamin D status: Study of 1326 cases from the UK Biobank. Journal of Public Health. https://doi.org/10.1093/pubmed/fdaa095

Rajter, J. C., Sherman, M., Fatteh, N., Vogel, F., Sacks, J., \& Rajter, J.-J. (2020). ICON (Ivermectin in COvid Nineteen) study: Use of Ivermectin is Associated with Lower Mortality in Hospitalized Patients with COVID19. medRxiv, 2020.06.06.20124461. https://doi.org/10.1101/2020.06.06.20124461

Ramachandran, P., Kathirvelu, B., Chakraborti, A., Gajendran, M., Zhahid, U., Ghanta, S., Onukogu, I., Narh, J. T., Wang, J. C., \& Anwer, F. (2020). COVID-19 in Cancer Patients From New York City: A Comparative Single Center Retrospective Analysis. Cancer Control, 27(1), 107327482096045. https://doi.org/10.1177/1073274820960457

Ramlall, V., Thangaraj, P., Meydan, C., Foox, J., Butler, D., May, B., Freitas, J. de, Glicksberg, B. S., Mason, C., Tatonetti, N. P., \& Shapira, S. D. (2020). Identification of Immune complement function as a determinant of adverse SARS-CoV-2 infection outcome. medRxiv, 2020.05.05.20092452. https://doi.org/10.1101/2020.05.05.20092452

Rashid, M., Wu, J., Timmis, A., Curzen, N., Zaman, A., Clarke, S., Nolan, J., Shoiab, A., Mohamed, M. O., Belder, M. D., Deanfield, J., Gale, C., \& Mamas, M. (2020). Clinical Characteristics and Outcomes of COVID-19 Positive Acute Coronary Syndrome Patients; a multisource Electronic Healthcare Records Study from England. medRxiv, 2020.08.20.20175091. https://doi.org/10.1101/2020.08.20.20175091

Rashid, R. A., Zgair, A., \& Al-Ani, R. M. (2021). Effect of nasal corticosteroid in the treatment of anosmia due to COVID-19: A randomised double-blind placebo-controlled studyAmerican Journal of Otolaryngology, 42(5), 103033. https://doi.org/10.1016/j.amjoto.2021.103033

Ravindra, G., Chitra, L., Madhur, M., Santosh, D., Venugopalan, P., Sundeep, S., Shreepad, B., Piyush, C., Pradeep, D., Ashwini, J., Abhay, M., Vikram, P., Chetan, P., \& Urvi, S. (2021). Retrospective Assessment of Treatments of Hospitalized Covid-19 Patients[Preprint]. Epidemiology. https://doi.org/10.1101/2021.04.20.21255792 
Reese, J. T., Coleman, B., Chan, L., Blau, H., Callahan, T. J., Cappelletti, L., Fontana, T., Bradwell, K. R., Harris, N. L., Casiraghi, E., Valentini, G., Karlebach, G., Deer, R., McMurry, J. A., Haendel, M. A., Chute, C. G., Pfaff, E., Moffitt, R., Spratt, H., ... Robinson, P. N. (2021). Cyclooxygenase inhibitor use is associated with increased COVID-19 severity[Preprint]. Infectious Diseases (except HIV/AIDS). https://doi.org/10.1101/2021.04.13.21255438

Regina, J., Papadimitriou-Olivgeris, M., Burger, R., Filippidis, P., Tschopp, J., Desgranges, F., Viala, B., Kampouri, E., Rochat, L., Haefliger, D., Belkoniene, M., Fidalgo, C., Kritikos, A., Jaton, K., Senn, L., Bart, P.-A., Pagani, J.-L., Manuel, O., \& Lhopitallier, L. (2020). Epidemiology, risk factors and clinical course of SARS-CoV-2 infected patients in a Swiss university hospital: An observational retrospective study. medRxiv, 2020.05.11.20097741. https://doi.org/10.1101/2020.05.11.20097741

Reiter, T., Pajenda, S., Wagner, L., Gaggl, M., Atamaniuk, J., Holzer, B., Zimpernik, I., Gerges, D., Mayer, K., Aigner, C., Strassl, R., Jansen-Skoupy, S., Födinger, M., Sunder-Plassmann, G., \& Schmidt, A. (2020). Covid-19 serology in nephrology health care workers. medRxiv, 2020.07.21.20136218. https://doi.org/10.1101/2020.07.21.20136218

Ren, H. G., Guo, X., Blighe, K., Zhu, F., Martin, J., Safdar, L. B., Yang, P., Wang, D. W., Hu, Q., Huo, N., Stebbing, J., \& Cheng, D. (2020). Risk Factors for ICU Admission, Mechanical Ventilation and Mortality in Hospitalized Patients with COVID-19 in Hubei, China. medRxiv, 2020.08.31.20184952. https://doi.org/10.1101/2020.08.31.20184952

Ren, H. G., Guo, X., Tu, L., Hu, Q., Blighe, K., Safdar, L. B., Stebbing, J., Weiner, S. D., Willis, M. S., Rosendaal, F. R., Xu, G., Cao, F., \& Wang, D. W. (2020)Clinical Characteristics and Risk Factors for Myocardial Injury and Arrhythmia in COVID-19 patients [Preprint]. Cardiovascular Medicine.https://doi.org/10.1101/2020.11.30.20190926

Rentsch, Christopher T., Beckman, J. A., Tomlinson, L., Gellad, W. F., Alcorn, C., Kidwai-Khan, F., Skanderson, M., Brittain, E., King, J. T., Ho, Y.-L., Eden, S., Kundu, S., Lann, M. F., Greevy, R. A., Ho, P. M., Heidenreich, P. A., Jacobson, D. A., Douglas, I. J., Tate, J. P., ... Freiberg, M. S. (2020a). Early initiation of prophylactic anticoagulation for prevention of COVID-19 mortality: A nationwide cohort study of hospitalized patients in the United States [Preprint]. Infectious Diseases (except HIV/AIDS).https://doi.org/10.1101/2020.12.09.20246579

Rentsch, Christopher T., Kidwai-Khan, F., Tate, J. P., Park, L. S., King, J. T., Skanderson, M., Hauser, R. G., Schultze, A., Jarvis, C. I., Holodniy, M., Re, V. L., Akgun, K. M., Crothers, K., Taddei, T. H., Freiberg, M. S., \& Justice, A. C. (2020b). Covid-19 Testing, Hospital Admission, and Intensive Care Among 2,026,227 United States Veterans Aged 54-75 Years. medRxiv, 2020.04.09.20059964. https://doi.org/10.1101/2020.04.09.20059964

Rica, R. de la, Borges, M., Aranda, M., Castillo, A. del, Socias, A., Payeras, A., Rialp, G., Socias, L., Masmiquel, L., \& Gonzalez-Freire, M. (2020). Low albumin levels are associated with poorer outcomes in a case series of COVID-19 patients in Spain: A retrospective cohort study. medRxiv, 2020.05.07.20094987. https://doi.org/10.1101/2020.05.07.20094987

Richard, A., Wisniak, A., Perez-Saez, J., Garrison-Desany, H., Petrovic, D., Piumatti, G., Baysson, H., Picazio, A., Pennacchio, F., Ridder, D. D., Chappuis, F., Vuilleumier, N., Low, N., Hurst, S., Eckerle, I., Flahault, A., Kaiser, L., Azman, A. S., Guessous, I., ... Group, F. the S.-P. study. (2020). Seroprevalence of anti-SARS-CoV-2 IgG antibodies, risk factors for infection and associated symptoms in Geneva, Switzerland: A population-based study. medRxiv, 2020.12.16.20248180. https://doi.org/10.1101/2020.12.16.20248180

Richardson, S., Hirsch, J. S., Narasimhan, M., Crawford, J. M., McGinn, T., Davidson, K. W., Barnaby, D. P., Becker, L. B., Chelico, J. D., Cohen, S. L., Cookingham, J., Coppa, K., Diefenbach, M. A., Dominello, A. J., Duer-Hefele, J., Falzon, L., Gitlin, J., Hajizadeh, N., Harvin, T. G., ... Zanos, T. P. (2020). Presenting Characteristics, Comorbidities, and Outcomes Among 5700 Patients Hospitalized With COVID-19 in the New York City Area. JAMA, 323(20), 2052-2059. https://doi.org/10.1001/jama.2020.6775

Riley, S., Ainslie, K. E. C., Eales, O., Jeffrey, B., Walters, C. E., Atchison, C. J., Diggle, P. J., Ashby, D., Donnelly, C. A., Cooke, G., Barclay, W., Ward, H., Taylor, G., Darzi, A., \& Elliott, P. (2020). Community prevalence of SARS-CoV-2 virus in England during May 2020: REACT study. medRxiv, 2020.07.10.20150524. https://doi.org/10.1101/2020.07.10.20150524

Rimland, C. A., Morgan, C. E., Bell, G. J., Kim, M. K., Hedrick, T., Marx, A., Bramson, B., Swygard, H., Napravnik, S., Schmitz, J. L., Carson, S. S., Fischer, W. A., Eron, J. J., Gay, C. L., \& Parr, J. B. (2020). Clinical characteristics and early outcomes in patients with COVID-19 treated with tocilizumab at a United States academic center. medRxiv, 2020.05.13.20100404. https://doi.org/10.1101/2020.05.13.20100404

Riou, M., Marcot, C., Canuet, M., Renaud-Picard, B., Chatron, E., Porzio, M., Dégot, T., Hirschi, S., Metz-Favre, C., Kassegne, L., Ederle, C., Khayath, N., Labani, A., Leyendecker, P., De Blay, F., \& Kessler, R. (2021). Clinical characteristics of and outcomes for patients with COVID-19 and comorbid lung diseases primarily hospitalized in a conventional pulmonology unit: A retrospective study. Respiratory Medicine and Research, 79, 100801. https://doi.org/10.1016/i.resmer.2020.100801

Riyahi, S., Dev, H., Behzadi, A., Kim, J., Attari, H., Raza, S. I., Margolis, D. J., Jonisch, A., Megahed, A., Bamashmos, A., Elfatairy, K., \& Prince, M. R. (2021). Pulmonary Embolism in Hospitalized Patients with COVID-19: A Multicenter Study. Radiology, 210777. https://doi.org/10.1148/radiol.2021210777

Rizzo, S., Chawla, D., Zalocusky, K., Keebler, D., Chia, J., Lindsay, L., Yau, V., Kamath, T., \& Tsai, L. (2020). Descriptive epidemiology of 16,780 hospitalized COVID-19 patients in the United States. medRxiv, 2020.07.17.20156265. https://doi.org/10.1101/2020.07.17.20156265

Robilotti, E. V., Babady, N. E., Mead, P. A., Rolling, T., Perez-Johnston, R., Bernardes, M., Bogler, Y., Caldararo, M., Figueroa-Ortiz, C., Glickman, M., Joanow, A., Kaltsas, A., Lee, Y. J., Bianchi, A. L., Mariano, A., Morjaria, S., Nawar, T., Papanicolaou, G. A., Predmore, J., ... Kamboj, M. (2020). Determinants of Severity in Cancer Patients with COVID-19 Illness. medRxiv, 2020.05 .04 .20086322$. https://doi.org/10.1101/2020.05.04.20086322

Robinson, L., Wang, L., Fu, X., Wallace, Z., Long, A., Zhang, Y., Camargo, C., \& Blumenthal, K. (2021). COVID-19 severity in asthma patients: A multi-center matched cohort studyJournal of Allergy and Clinical Immunology, 147(2), AB241. https://doi.org/10.1016/j.jaci.2020.12.026

Roederer, T., Mollo, B., Vincent, C., Nikolay, B., Llosa, A. E., Nesbitt, R., Vanhomwegen, J., Rose, T., Goyard, S., Anna, F., Torre, C., Fourrey, E., Simons, E., Hennequin, W., Mills, C., \& Luquero, F. J. (2021). Seroprevalence and risk factors of exposure to COVID-19 in homeless people in Paris, France: A cross-sectional study. The Lancet Public Health, S2468266721000013. https://doi.org/10.1016/S2468-2667(21)00001-3

Rogier, T., Eberl, I., Moretto, F., Sixt, T., Catherine, F.-X., Estève, C., Abdallahoui, M., Behague, L., Coussement, A., Mathey, L., Mahy, S., Buisson, M., Salmon-Rousseau, A., Duong, M., Chavanet, P., Bernard, Q., Nicolas, B., Benguella, L., Bonnotte, B., ... Piroth, L. (2021). COVID-19 or not COVID-19? Compared characteristics of patients hospitalized for suspected COVID-19. European Journal of Clinical Microbiology \& Infectious Diseases. https://doi.org/10.1007/s10096-021-04216-3

Romão, V. C., Oliveira-Ramos, F., Cruz-Machado, A. R., Martins, P., Barreira, S., Silva-Dinis, J., Galaio, L., Proença, H., Cristino, J. M., Sacadura-Leite, E., Khmelinskii, N., Romeu, J. C., Fonseca, 
J. E., \& Department, C. R. (2020). A COVID-19 outbreak in a rheumatology department upon the early days of the pandemic. medRxiv, 2020.06.05.20107011. https://doi.org/10.1101/2020.06.05.20107011

Rossi, B., Nguyen, L. S., Zimmermann, P., Boucenna, F., Baucher, L., Dubret, L., Guillot, H., Bouldouyre, M., Allenbach, Y., Salem, J.-E., Barsoum, P., Oufella, A., \& Gros, H. (2020). Effect of tocilizumab in hospitalized patients with severe pneumonia COVID-19: A cohort study. medRxiv, 2020.06.06.20122341. https://doi.org/10.1101/2020.06.06.20122341

Rowlands, A. V., Gillies, C., Chudasama, Y., Davies, M. J., Islam, N., Kloecker, D. E., Lawson, C., Pareek, M., Razieh, C., Zaccardi, F., Yates, T., \& Khunti, K. (2020)Association of working shifts, inside and outside of healthcare, with risk of severe COVID-19: An observational study. https://doi.org/10.1101/2020.12.16.20248243

Röthlin Eriksen, A. R., Fogh, K., Hasselbalch, R. B., Bundgaard, H., Nielsen, S. D., Jørgensen, C. S., Scharff, B. F., Erikstrup, C., Sækmose, S. G., Holm, D. K., Aagaard, B., Kristensen, J. H., Bødker, C. A., Norsk, J., Nielsen, P. B., Østergaard, L., Ellermann-Eriksen, S., Andersen, B., Nielsen, H., ... Iversen, K. (2021). SARS-CoV-2 antibody prevalence among homeless people, sex workers and shelter workers in Denmark: A nationwide cross-sectional study [Preprint]. Epidemiology. https://doi.org/10.1101/2021.05.07.21256388

Rubina, K., Shmakova, A., Shabanov, A., Andreev, Yu., Borovkova, N., Kulabukhov, V., Evseev, A., Popugaev, K., Petrikov, S., \& Semina, E. (2021)Novel prognostic determinants of COVID-19related mortality: A pilot study on severely-ill patients in Russia [Preprint]. Infectious Diseases (except HIV/AIDS).https://doi.org/10.1101/2021.04.01.21254688

Rubio-Rivas, M., Ronda, M., Padulles, A., Mitjavila, F., Riera-Mestre, A., Garcia-Forero, C., Iriarte, A., Mora, J. M., Padulles, N., Gonzalez, M., Solanich, X., Gasa, M., Suarez, G., Sabater, J., PerezFernandez, X. L., Santacana, E., Leiva, E., Ariza-Sole, A., Dallaglio, P. D., ... Corbella, X. (2020). Beneficial Effect of Corticosteroids in Preventing Mortality in Patients Receiving Tocilizumab to Treat Severe COVID-19 IIIness. medRxiv, 2020.08.31.20182428. https://doi.org/10.1101/2020.08.31.20182428

Russell, B., Moss, C., Papa, S., Irshad, S., Ross, P., Spicer, J., Kordasti, S., Crawley, D., Wylie, H., Cahill, F., Haire, A., Zaki, K., Rahman, F., Sita-Lumsden, A., Josephs, D., Enting, D., Lei, M., Ghosh, S., Harrison, C., ... Van Hemelrijck, M. (2020). Factors Affecting COVID-19 Outcomes in Cancer Patients: A First Report From Guy's Cancer Center in London. Frontiers in Oncology, 10 , 1279. https://doi.org/10.3389/fonc.2020.01279

Saeed, G. A., Al Helali, A. A., Almazrouei, S., Shah, A., \& Ahmed, L. A. (2020).Chest CT features of COVID-19 in the region of Abu Dhabi, UAE- A single institute studyiPreprint]. Radiology; Imaging. https://doi.org/10.1101/2020.11.14.20229096

Salama, C., Han, J., Yau, L., Reiss, W. G., Kramer, B., Neidhart, J. D., Criner, G. J., Kaplan-Lewis, E., Baden, R., Pandit, L., Cameron, M. L., Garcia-Diaz, J., Chávez, V., Mekebeb-Reuter, M., Lima de Menezes, F., Shah, R., González-Lara, M. F., Assman, B., Freedman, J., \& Mohan, S. V. (2021). Tocilizumab in Patients Hospitalized with Covid-19 Pneumonia. New England Journal of Medicine, 384(1), 20-30. https://doi.org/10.1056/NEJMoa2030340

Salerno, S., Zhao, Z., Sankar, S. P., Salvatore, M., Gu, T., Fritsche, L. G., Lee, S., Lisabeth, L. D., Valley, T. S., \& Mukherjee, B. (2020). Understanding the patterns of repeated testing for COVID19: Association with patient characteristics and outcomes. medRxiv, 2020.07.26.20162453. https://doi.org/10.1101/2020.07.26.20162453

Salton, F., Confalonieri, P., Santus, P., Harari, S., Scala, R., Lanini, S., Vertui, V., Oggionni, T., Caminati, A., Patruno, V., Tamburrini, M., Scartabellati, A., Parati, M., Villani, M., Radovanovic, D., Tomassetti, S., Ravaglia, C., Poletti, V., Vianello, A., ... Confalonieri, M. (2020). Prolonged low-dose methylprednisolone in patients with severe COVID-19 pneumonia. medRxiv, 2020.06.17.20134031. https://doi.org/10.1101/2020.06.17.20134031

Sami, R., Soltaninejad, F., Amra, B., Naderi, Z., Javanmard, S. H., Iraj, B., Ahmadi, S. H., Shayganfar, A., Dehghan, M., Khademi, N., Hosseini, N. S., Mortazavi, M., Mansourian, M., Mananas, M. A., Marateb, H. R., \& Adibi, P. (2020). A one-year hospital-based prospective COVID-19 open-cohort in the Eastern Mediterranean region: The Khorshid COVID Cohort (KCC) study. medRxiv, 2020.05.11.20096727. https://doi.org/10.1101/2020.05.11.20096727

Sandri, M. T., Azzolini, E., Torri, V., Carloni, S., Pozzi, C., Salvatici, M., Tedeschi, M., Castoldi, M., Mantovani, A., \& Rescigno, M. (2021). SARS-CoV-2 serology in 4000 health care and administrative staff across seven sites in Lombardy, Italy. Scientific Reports, 11(1), 12312. https://doi.org/10.1038/s41598-021-91773-4

Santos, C., Rhee, Y., Hollinger, E., Olaitan, O., Schadde, E., Peev, V., Saltzberg, S., \& Hertl, M. (2020). Comparative Incidence and Outcomes of COVID-19 in Kidney or Kidney-Pancreas Transplant Recipients Versus Kidney or Kidney-Pancreas Waitlisted Patients: A Pilot Study. medRxiv, 2020.07.20.20157990. https://doi.org/10.1101/2020.07.20.20157990

Saurabh, S., Verma, M. K., Gautam, V., Kumar, N., Jain, V., Goel, A. D., Gupta, M. K., Sharma, P. P., Bhardwaj, P., Singh, K., Nag, V. L., Garg, M. K., \& Misra, S. (2021). Tobacco, alcohol use and other risk factors for developing symptomatic COVID-19 vs asymptomatic SARS-CoV-2 infection: A case-control study from western Rajasthan, India. Trans $R$ Soc Trop Med Hyg, NA. https://doi.org/10.1093/trstmh/traa172

Savarraj, J. P., Burkett, A. B., Hinds, S. N., Paz, A. S., Assing, A., Juneja, S., Colpo, G. D., Torres, L. F., Gusdon, A., McCullough, L., \& Choi, H. A. (n.d.)Three-month outcomes in hospitalized COVID-19 patients. 7. https://doi.org/10.1101/2020.10.16.20211029

Schneeweiss, M. C., Leonard, S., Weckstein, A., Schneeweiss, S., \& Rassen, J. (2020). Renin-Angiotensin-Aldosterone-System inhibitor use in patients with CoVID-19 infection and prevention of serious events: A cohort study in commercially insured patients in the US. medRxiv, 2020.07.22.20159855. https://doi.org/10.1101/2020.07.22.20159855

Schoeman, D., \& Fielding, B. C. (2019). Coronavirus envelope protein: Current knowledge.Virology Journal, 16(1), 69. https://doi.org/10.1186/s12985-019-1182-0

Schubl, S. D., Figueroa, C., Palma, A. M., Assis, R. R. de, Jain, A., Nakajima, R., Jasinkas, A., Brabender, D., Naaseh, A., Dominguez, O. H., Runge, A., Skochko, S., Chinn, J., Kelsey, A. J., Lai, K. T., Zhao, W., Horvath, P., Tifrea, D., Grigorian, A., ... Khan, S. (2020). Risk Factors for SARS-CoV-2 Seropositivity in a Health Care Worker Population https://doi.org/10.1101/2020.12.17.20248430

Scully, E. P., Schumock, G., Fu, M., Massaccesi, G., Muschelli, J., Betz, J., Klein, E. Y., West, N. E., Robinson, M., Garibaldi, B. T., Bandeen-Roche, K., Zeger, S., Klein, S. L., Gupta, A., \& for the $\mathrm{JH}-\mathrm{CROWN}$ registry team. (2021). Sex and gender differences in COVID testing, hospital admission, presentation, and drivers of severe outcomes in the DC/Maryland regiotPreprint]. Infectious Diseases (except HIV/AIDS). https://doi.org/10.1101/2021.04.05.21253827

Senkal, N. (2020). Chronic ACE Inhibitor use is Associated with Decreased Odds of Severe Disease in Patients with COVID-19.The Anatolian Journal of Cardiology. https://doi.org/10.14744/AnatolJCardiol.2020.57431 
Serling-Boyd, N., D'Silva, K. M., Hsu, T. Y., Wallwork, R., Fu, X., Gravallese, E. M., Jorge, A. M., Zhang, Y., Choi, H., Sparks, J. A., \& Wallace, Z. S. (2020). Coronavirus disease 2019 outcomes among patients with rheumatic diseases 6 months into the pandemic. Annals of the Rheumatic Diseases, annrheumdis-2020-219279. https://doi.org/10.1136/annrheumdis-2020-219279

Shade, J. K., Doshi, A. N., Sung, E., Popescu, D. M., Minhas, A. S., Gilotra, N. A., Aronis, K. N., Hays, A. G., \& Trayanova, N. A. (2021)COVID-HEART: Development and Validation of a MultiVariable Model for Real-Time Prediction of Cardiovascular Complications in Hospitalized Patients with COVID-19. https://doi.org/10.1101/2021.01.03.21249182

Shah, S. J., Barish, P. N., Prasad, P. A., Kistler, A. L., Neff, N., Kamm, J., Li, L. M., Chiu, C. Y., Babick, J. M., Fang, M. C., Abe-Jones, Y., Alipanah, N., Alvarez, F. N., Botvinnik, O. B., Davis, J. M., Castenada, G. D., Consortium, C., Dadasovich, R. M., Deng, X., ... Langelier, C. (2020). Clinical features, diagnostics, and outcomes of patients presenting with acute respiratory illness: A comparison of patients with and without COVID-19. medRxiv, 2020.05.02.20082461. https://doi.org/10.1101/2020.05.02.20082461

Shahab, L., Brose, L. S., \& West, R. (2013). Novel delivery systems for nicotine replacement therapy as an aid to smoking cessation and for harm reduction: Rationale, and evidence for advantages over existing systems. CNS Drugs, 27(12), 1007-1019. https://doi.org/10.1007/s40263-013-0116-4

Sharma, A. K., Ahmed, A., Baig, V. N., Dhakad, P., Dalela, G., Kacker, S., Panwar, V. R., Panwar, R. B., \& Gupta, R. (2020). Characteristics and Outcomes of Hospitalized Young Adults with Mild to Moderate Covid-19 at a University Hospital in India. medRxiv, 2020.06.02.20106310. https://doi.org/10.1101/2020.06.02.20106310

Shekhar, R., Sheikh, A. B., Upadhyay, S., Atencio, J., \& Kapuria, D. (2020). Early experience with COVID-19 patients at academic hospital in Southwestern United States/nfectious Diseases, 52(8), 596-599. https://doi.org/10.1080/23744235.2020.1774645

Shi, H., Zuo, Y., Yalavarthi, S., Gockman, K., Zuo, M., Madison, J. A., Blair, C. N., Woodard, W., Lezak, S. P., Lugogo, N. L., Woods, R. J., Lood, C., Knight, J. S., \& Kanthi, Y. (2020). Neutrophil calprotectin identifies severe pulmonary disease in COVID-19. medRxiv, 2020.05.06.20093070. https://doi.org/10.1101/2020.05.06.20093070

Shi, P., Ren, G., Yang, J., Li, Z., Deng, S., Li, M., Wang, S., Xu, X., Chen, F., Li, Y., Li, C., Yang, X., Xie, Z., Wu, Z., \& Chen, M. (2020). Clinical characteristics of imported and second-generation COVID-19 cases outside Wuhan, China: A multicenter retrospective study. medRxiv, 2020.04.19.20071472. https://doi.org/10.1101/2020.04.19.20071472

Shi, Q., Zhao, K., Yu, J., Jiang, F., Feng, J., Zhao, K., Zhang, X., Chen, X., Hu, P., Hong, Y., Li, M., Liu, F., Chen, C., \& Wang, W. (2020). Clinical characteristics of 101 COVID-19 nonsurvivors in Wuhan, China: A retrospective study. medRxiv, 2020.03.04.20031039. https://doi.org/10.1101/2020.03.04.20031039

Shi, Y., Yu, X., Zhao, H., Wang, H., Zhao, R., \& Sheng, J. (2020). Host susceptibility to severe COVID-19 and establishment of a host risk score: Findings of 487 cases outside WuhanCritical Care, 24(1), 108. https://doi.org/10.1186/s13054-020-2833-7

Shi, Z., Resurreccion, W. K., Wang, C.-H., Wei, J., Na, R., Zheng, S. L., Billings, L. K., Helfand, B. T., Khandekar, J., \& Xu, J. (2020). Association of Cancer with Risk and Mortality of COVID-19: Results from the UK Biobank. medRxiv, 2020.07.10.20151076. https://doi.org/10.1101/2020.07.10.20151076

Shields, A. M., Faustini, S. E., Kristunas, C. A., Cook, A. M., Backhouse, C., Dunbar, L., Ebanks, D., Emmanuel, B., Crouch, E., Kroeger, A., Hirschfeld, J., Sharma, P., Jaffery, R., Nowak, S., Gee, S., Drayson, M. T., Richter, A. G., Dietrich, T., \& Chapple, I. C. (2021). Longitudinal protection following natural SARS-CoV-2 infection and early vaccine responses: Insights from a cohort of community based dental health care professionals [Preprint]. Allergy; Immunology. https://doi.org/10.1101/2021.02.24.21252368

Shiri, A. H., Raiatdoost, E., Afkhami, H., Ravanshad, R., Hosseini, S. E., Kalani, N., \& Raoufi, R. (2021).The herbal combination of Sugarcane, Black Myrobalan, and mastic as a supplementary treatment for COVID-19: A randomized clinical trial [Preprint]. Respiratory Medicine. https://doi.org/10.1101/2021.04.27.21256221

Sholzberg, M., Tang, G. H., Rahhal, H., AlHamzah, M., Kreuziger, L. B., Ní Áinle, F., Alomran, F., Alayed, K., Alsheef, M., AlSumait, F., Pompilio, C. E., Sperlich, C., Tangri, S., Tang, T., Jaksa, P., Suryanarayan, D., Almarshoodi, M., Castellucci, L., James, P. D., ... the RAPID Trial investigators. (2021). Heparin for Moderately III Patients with Covid-19[Preprint]. Hematology. https://doi.org/10.1101/2021.07.08.21259351

Sierpiński, R., Pinkas, J., Jankowski, M., Zgliczyński, W. S., Wierzba, W., Gujski, M., \& Szumowski, Ł. (2020). Sex differences in the frequency of gastrointestinal symptoms and olfactory or taste disorders among 1,942 non-hospitalized patients with COVID-19. Polish Archives of Internal Medicine https://doi.org/10.20452/pamw.15414

Sigel, K., Swartz, T., Golden, E., Paranjpe, I., Somani, S., Richter, F., De Freitas, J. K., Miotto, R., Zhao, S., Polak, P., Mutetwa, T., Factor, S., Mehandru, S., Mullen, M., Cossarini, F., Bottinger, E. Fayad, Z., Merad, M., Gnjatic, S., ... Glicksberg, B. S. (n.d.). Covid-19 and People with HIV Infection: Outcomes for Hospitalized Patients in New York City. Clinical Infectious Diseases. https://doi.org/10.1093/cid/ciaa880

Silva Neto, P. V. da, Carvalho, J. C. S. de, Pimentel, V. E., Perez, M. M., Carmona-Garcia, I., Neto, N. T., Toro, D. M., Oliveira, C. N. S., Fraga-Silva, T. F. C., Milanezi, C. M., Rodrigues, L. C., Dias, C. F. S. L., Xavier, A. C., Porcel, G. S., Guarneri, I. C., Zaparoli, K., Garbato, C. T., Argolo, J. G. M., Junior, A. A. F., ... Sorgi, C. A. (2020). Prognostic value of sTREM-1 in COVID-19 patients: A biomarker for disease severity and mortality [Preprint]. Infectious Diseases (except HIV/AIDS).https://doi.org/10.1101/2020.09.22.20199703

Simons, D., Brown, J., Shahab, L., \& Perski, O. (2020). Smoking and COVID-19: Rapid evidence review for the Royal College of Physicians, London (UK)Qeios. https://doi.org/10.32388/VGJCUN

Simons, D., Perski, O., Shahab, L., Brown, J., \& Bailey, R. (2020). The association of smoking status with hospitalisation for COVID-19 compared with other respiratory viruses a year previous: A case-control study at a single UK National Health Service trust. medRxiv, 2020.11.26.20238469. https://doi.org/10.1101/2020.11.26.20238469

Simons, D., Shahab, L., Brown, J., \& Perski, O. (2020). The association of smoking status with SARS-CoV-2 infection, hospitalisation and mortality from COVID-19: A living rapid evidence review with Bayesian meta-analyses (version 7). Addiction, n/a(n/a). https://doi.org/10.1111/add.15276

Singh, B. M., Bateman, J., Viswanath, A., Klaire, V., Mahmud, S., Nevill, A. M., \& Dunmore, S. J. (2020).The risk of COVID hospital admission and COVID mortality during the first COVID 19 wave with a special emphasis on Ethnic Minorities: An observational study of a single, deprived, multi ethnic UK health economy [Preprint]. Infectious Diseases (except HIV/AIDS).

https://doi.org/10.1101/2020.11.20.20224691

Sisó-Almirall, A., Kostov, B., Mas-Heredia, M., Vilanova-Rotllan, S., Sequeira-Aymar, E., Sans-Corrales, M., Sant-Arderiu, E., Cayuelas-Redondo, L., Martínez-Pérez, A., Plana, N. G., AnguitaGuimet, A., \& Benavent-Àreu, J. (2020). PROGNOSTIC FACTORS IN SPANISH COVID-19 PATIENTS: A CASE SERIES FROM BARCELONA. medRxiv, 2020.06.18.20134510. 
https://doi.org/10.1101/2020.06.18.20134510

Soares, R. de C. M., Mattos, L. R., \& Raposo, L. M. (2020).Risk Factors for Hospitalization and Mortality due to COVID-19 in Espírito Santo State, Brazil tpmd200483. https://doi.org/10.4269/ajtmh.20-0483

Solís, P., \& Carreño, H. (2020). COVID-19 Fatality and Comorbidity Risk Factors among Diagnosed Patients in Mexico https://doi.org/10.1101/2020.04.21.20074591

Sonnweber, T., Tymoszuk, P., Sahanic, S., Boehm, A., Pizzini, A., Luger, A., Schwabl, C., Nairz, M., Kurz, K., Koppelstätter, S., Aichner, M., Puchner, B., Egger, A., Hoermann, G., Wöll, E., Weiss, G., Widmann, G., Tancevski, I., \& Löffler-Ragg, J. (2021). Investigating phenotypes of pulmonary COVID-19 recovery - a longitudinal observational prospective multicenter trial[Preprint]. Epidemiology. https://doi.org/10.1101/2021.06.22.21259316

Soto-Mota, A., Garza, B. A. M., Rodriguez, E. M., Rodriguez, J. O. B., Romo, A. E. L., Minutti, P. A., Loya, J. V. A., Talavera, F. E. P., Avila-Cervera, F. J., Burciaga, A. N. V., Aramburo, O. M., Olguin, L. A. P., Soto-Rodriguez, A., Prado, A. C., Santillan-Doherty, P., Galindo, J. O. G., Gordillo, D. H., \& Mejia, J. G. (2020). THE LOW-HARM SCORE FOR PREDICTING MORTALITY IN PATIENTS DIAGNOSED WITH COVID-19: A MULTICENTRIC VALIDATION STUDY. medRxiv, 2020.05.26.20111120. https://doi.org/10.1101/2020.05.26.20111120

Sourij, H., Aziz, F., Bräuer, A., Ciardi, C., Clodi, M., Fasching, P., Karolyi, M., Kautzky-Willer, A., Klammer, C., Malle, O., Oulhaj, A., Pawelka, E., Peric, S., Ress, C., Sourij, C., Stechemesser, L., Stingl, H., Stulnig, T., Tripolt, N., ... for the COVID-19 in diabetes in Austria study group. (2020). COVID-19 fatality prediction in people with diabetes and prediabetes using a simple score upon hospital admission. Diabetes, Obesity and Metabolism, dom.14256. https://doi.org/10.1111/dom.14256

Souza, F. S. H., Hojo-Souza, N. S., Santos, E. B., Silva, C. M., \& Guidoni, D. L. (2020). Predicting the disease outcome in COVID-19 positive patients through Machine Learning: A retrospective cohort study with Brazilian data. medRxiv, 2020.06.26.20140764. https://doi.org/10.1101/2020.06.26.20140764

statistique, O. fédéral de la. (2020). La part des gros fumeurs s'est réduite de moitié en 25 ans - Enquête suisse sur la santé 2017: Consommation de tabac Communiqué de presse. II0ffice fédéral de la statistique. Lcontent/bfs/fr/home/aktuell/neue-veroeffentlichungen.assetdetail.11907023.html

Stead, L. F., Buitrago, D., Preciado, N., Sanchez, G., Hartmann-Boyce, J., \& Lancaster, T. (2013). Physician advice for smoking cessation Cochrane Database of Systematic Reviews 2017(12). https://doi.org/10.1002/14651858.CD000165.pub4

Sterlin, D., Mathian, A., Miyara, M., Mohr, A., Anna, F., Claer, L., Quentric, P., Fadlallah, J., Ghillani, P., Gunn, C., Hockett, R., Mudumba, S., Guihot, A., Luyt, C.-E., Mayaux, J., Beurton, A., Fourati, S., Lacorte, J.-M., Yssel, H., ... Gorochov, G. (2020). IgA dominates the early neutralizing antibody response to SARS-CoV-2. medRxiv, 2020.06.10.20126532. https://doi.org/10.1101/2020.06.10.20126532

Strangfeld, A., Schafer, M., Gianfrancesco, M. A., Lawson-Tovey, S., Liew, J. W., Ljung, L., Mateus, E. F., Richez, C., Santos, M. J., Schmajuk, G., Scire, C. A., Sirotich, E., Sparks, J. A., Sufka, P., Thomas, T., Trupin, L., Wallace, Z. S., Al-Adely, S., Bachiller-Corral, J., ... COVID-19 Global Rheumatology Alliance Consortium. (2021). Factors associated with COVID-19-related death in people with rheumatic diseases: Results from the COVID-19 Global Rheumatology Alliance physician-reported registry. Ann Rheum Dis, NA. https://doi.org/10.1136/annrheumdis-2020-219498

Suleyman, G., Fadel, R. A., Malette, K. M., Hammond, C., Abdulla, H., Entz, A., Demertzis, Z., Hanna, Z., Failla, A., Dagher, C., Chaudhry, Z., Vahia, A., Lanfranco, O. A., Ramesh, M., Zervos, M. J., Alangaden, G., Miller, J., \& Brar, I. (2020). Clinical Characteristics and Morbidity Associated With Coronavirus Disease 2019 in a Series of Patients in Metropolitan Detroit. JAMA Network Open, 3(6), e2012270-e2012270. https://doi.org/10.1001/jamanetworkopen.2020.12270

Sun, L., Surya, S., Le, A. N., Desai, H., Doucette, A., Gabriel, P., Ritchie, M. D., Rader, D., Maillard, I., Bange, E., Huang, A. C., Vonderheide, R. H., DeMichele, A., Verma, A., Mamtani, R., \& Maxwell, K. N. (2021). Rates of COVID-19-Related Outcomes in Cancer Compared With Noncancer Patients. JNCI Cancer Spectr, 5. https://doi.org/10.1093/jncics/pkaa120

Talavera, B., García-Azorín, D., Martínez-Pías, E., Trigo, J., Hernández-Pérez, I., Valle-Peñacoba, G., Simón-Campo, P., Lera, M. de, Chavarría-Miranda, A., López-Sanz, C., Gutiérrez-Sánchez, M., Martínez-Velasco, E., Pedraza, M., Sierra, Á., Gómez-Vicente, B., Guerrero, Á., \& Arenillas, J. F. (2020). Anosmia is associated with lower in-hospital mortality in COVID-19. Journal of the Neurological Sciences, 419, 117163. https://doi.org/10.1016/j.jns.2020.117163

Tao, P.-Y., Leng, L., Liu, K., Zhou, R.-H., Hu, Y.-C., Wu, S.-J., Xiao, Y.-D., \& Liu, J. (2020). Determination of risk factors for predicting the onset of symptoms in asymptomatic COVID-19 infected patients. International Journal of Medical Sciences, 17(14), 2187-2193. https://doi.org/10.7150/ijms.47576

Tardif, J.-C., Bouabdallaoui, N., L’Allier, P. L., Gaudet, D., Shah, B., Pillinger, M. H., Lopez-Sendon, J., Luz, P. da, Verret, L., Audet, S., Dupuis, J., Denault, A., Pelletier, M., Tessier, P. A., Samson, S., Fortin, D., Tardif, J.-D., Busseuil, D., Goulet, E., ... for the COLCORONA Investigators. (2021). Efficacy of Colchicine in Non-Hospitalized Patients with COVID-19 https://doi.org/10.1101/2021.01.26.21250494

Targher, G., Mantovani, A., Wang, X.-B., Yan, H.-D., Sun, Q.-F., Pan, K.-H., Byrne, C. D., Zheng, K. I., Chen, Y.-P., Eslam, M., George, J., \& Zheng, M.-H. (2020). Patients with diabetes are at higher risk for severe illness from COVID-19. Diabetes \& Metabolism. https://doi.org/10.1016/j.diabet.2020.05.001

Tarifi, A., Al Shdaifat, A. A., Al-Shudifat, A. M., Azab, M., Ismail, J., Bashir, R., Amro, A., Altarifi, A., \& Khader, Y. (2021). Clinical, sinonasal, and long-term smell and taste outcomes in mildly symptomatic COVID-19 patients. International Journal of Clinical Practice, 75(7). https://doi.org/10.1111/ijcp.14260

Tavakol, Z., Ghannadi, S., Tabesh, M. R., Halabchi, F., Noormohammadpour, P., Akbarpour, S., Alizadeh, Z., Nezhad, M. H., \& Reyhan, S. K. (2021). Relationship between physical activity, healthy lifestyle and COVID-19 disease severity; a cross-sectional study. J. Public Health, NA, 1-9. https://doi.org/10.1007/s10389-020-01468-9

Team, R. C. (2013). The R Project for Statistical Computing. 1-12.

Tehrani, D., Wang, X., Rafique, A. M., Hayek, S. S., Herrmann, J., Neilan, T. G., Desai, P., Morgans, A., Lopez-Mattei, J., Parikh, R. V., \& Yang, E. H. (2021a)lmpact of Cancer and Cardiovascular Disease on In-Hospital Outcomes of COVID-19 Patients: Results From the American Heart Association COVID-19 Cardiovascular Disease Registry [Preprint]. In Review. https://doi.org/10.21203/rs.3.rs-600795/v1

Tehrani, D., Wang, X., Rafique, A. M., Hayek, S. S., Herrmann, J., Neilan, T. G., Desai, P., Morgans, A., Lopez-Mattei, J., Parikh, R. V., \& Yang, E. H. (2021b)Impact of Cancer and Cardiovascular 
Disease on In-Hospital Outcomes of COVID-19 Patients: Results From the American Heart Association COVID-19 Cardiovascular Disease Registry [Preprint]. In Review. https://doi.org/10.21203/rs.3.rs-600795/v1

Thakur, K., Sagayaraj, A., Prasad, K. C., \& Gupta, A. (2021). Olfactory Dysfunction in COVID-19 Patients: Findings from a Tertiary Rural Centrelndian j. Otolaryngol. Head Neck Surg, NA, 1-7. https://doi.org/10.1007/s12070-021-02364-8

The CONCOR-1 Study Group, CONCOR-1 writing committee, Bégin, P., Callum, J., Jamula, E., Cook, R., Heddle, N. M., Tinmouth, A., Zeller, M. P., Beaudoin-Bussières, G., Amorim, L., Bazin, R., Loftsgard, K. C., Carl, R., Chassé, M., Cushing, M. M., Daneman, N., Devine, D. V., Dumaresq, J., ... for The CONCOR-1 Study Group. (2021). Convalescent plasma for hospitalized patients with COVID-19 and the effect of plasma antibodies: A randomized controlled, open-label trial [Preprint]. Infectious Diseases (except HIV/AIDS).https://doi.org/10.1101/2021.06.29.21259427

The OpenSAFELY Collaborative, Williamson, E. J., Tazare, J., Bhaskaran, K., McDonald, H. I., Walker, A. J., Tomlinson, L., Wing, K., Bacon, S., Bates, C., Curtis, H. J., Forbes, H., Minassian, C., Morton, C. E., Nightingale, E., Mehrkar, A., Evans, D., Nicholson, B. D., Leon, D., ... Goldacre, B. (2021). Predicting COVID-19 related death using the OpenSAFELY platform[Preprint]. Infectious Diseases (except HIV/AIDS). https://doi.org/10.1101/2021.02.25.21252433

Thiabaud, A., Iten, A., Balmelli, C., Senn, L., Troillet, N., Widmer, A., Flury, D., Schreiber, P. W., Vázquez, M., Damonti, L., Buettcher, M., Vuichard-Gysin, D., Kuhm, C., Cusini, A., Riedel, T., Nussbaumer, Y., Gaudenz, R., Heininger, U., Berger, C., ... Keiser, O. (2020). SARS-CoV-2/COVID-19 hospitalised patients in Switzerland: A prospective cohort profile[Preprint]. Epidemiology. https://doi.org/10.1101/2020.12.10.20246884

Thomas, D. R., Fina, L. H., Adamson, J. P., Sawyer, C., Jones, A., Nnoaham, K., Barrasa, A., Shankar, A. G., \& Williams, C. J. (2021)Social, demographic and behavioural determinants of SARSCoV-2 infection: A case-control study carried out during mass community testing of asymptomatic individuals in South Wales, December 2020 [Preprint]. Epidemiology. https://doi.org/10.1101/2021.04.06.21253465

Thompson, J. V., Meghani, N., Powell, B. M., Newell, I., Craven, R., Skilton, G., Bagg, L. J., Yaqoob, I., Dixon, M. J., Evans, E. J., Kambele, B., Rehman, A., \& Kwong, G. N. M. (2020). Patient characteristics and predictors of mortality in 470 adults admitted to a district general hospital in England with Covid-19. medRxiv, 2020.07.21.20153650. https://doi.org/10.1101/2020.07.21.20153650

Torres-Macho, J., Ryan, P., Valencia, J., Pérez-Butragueño, M., Jiménez, E., Fontán-Vela, M., Izquierdo-García, E., Fernandez-Jimenez, I., Álvaro-Alonso, E., Lazaro, A., Alvarado, M., Notario, H., Resino, S., Velez-Serrano, D., \& Meca, A. (2020). The PANDEMYC Score. An Easily Applicable and Interpretable Model for Predicting Mortality Associated With COVID-19. Journal of Clinical Medicine, 9(10), 3066. https://doi.org/10.3390/jcm9103066

Tricco, A. C., Antony, J., Zarin, W., Strifler, L., Ghassemi, M., Ivory, J., Perrier, L., Hutton, B., Moher, D., \& Straus, S. E. (2015). A scoping review of rapid review methodsBMC Medicine, 13(1), 224. https://doi.org/10.1186/s12916-015-0465-6

Trubiano, J. A., Vogrin, S., Smibert, O. C., Marhoon, N., Alexander, A. A., Chua, K. Y., James, F. L., Jones, N. R., Grigg, S. E., Xu, C. L., Moini, N., Stanley, S. R., Birrell, M. T., Rose, M. T., Gordon, C. L., Kwong, J. C., \& Holmes, N. E. (2020). COVID-MATCH65 - A prospectively derived clinical decision rule for severe acute respiratory syndrome coronavirus 2. medRxiv, 2020.06 .30 .20143818$. https://doi.org/10.1101/2020.06.30.20143818

Ugur Chousein, E. G., Cortuk, M., Cinarka, H., Tanriverdi, E., Turan, D., Yildirim, B. Z., Sezen, C. B., \& Ozgul, M. A. (2020). Is there any effect of smoking status on severity and mortality of hospitalized patients with COVID-19 pneumonia? Tuberk. Toraks, 68, 371-378. https://doi.org/10.5578/tt.70352

Ullah, A. Z. M. D., Sivapalan, L., Chelala, C., \& Kocher, H. M. (2020). COVID-19 in patients with hepatobiliary and pancreatic diseases in East London: A single-centre cohort studymedRxiv, 2020.09.07.20189621. https://doi.org/10.1101/2020.09.07.20189621

Vahidy, F. S., Pan, A. P., Ahnstedt, H., Munshi, Y., Choi, H. A., Tiruneh, Y., Nasir, K., Kash, B. A., Andrieni, J. D., \& McCullough, L. D. (2021). Sex differences in susceptibility, severity, and outcomes of coronavirus disease 2019: Cross-sectional analysis from a diverse US metropolitan area. PloS One, 16. https://doi.org/10.1371/journal.pone.0245556

Valenti, L., Bergna, A., Pelusi, S., Facciotti, F., Lai, A., Tarkowski, M., Berzuini, A., Caprioli, F., Santoro, L., Baselli, G., Ventura, C. D., Erba, E., Bosari, S., Galli, M., Zehender, G., \& Prati, D. (2020). SARS-CoV-2 seroprevalence trends in healthy blood donors during the COVID-19 Milan outbreak. medRxiv, 2020.05.11.20098442. https://doi.org/10.1101/2020.05.11.20098442

Valenzuela, O., Ibanez, S. E., Poli, M., Roessler, P., Aylwin, M., Roizen, G., Iruretagoyena, M., Agar, V., Donoso, J., Fierro, M., \& Montes, J. (2020). First report of tocilizumab use in a cohort of Latin American patients hospitalized for severe COVID-19 pneumonia. medRxiv, 2020.08.12.20173104. https://doi.org/10.1101/2020.08.12.20173104

Valle, D. M. D., Kim-schulze, S., Hsin-hui, H., Beckmann, N. D., Nirenberg, S., Wang, B., Lavin, Y., Swartz, T., Madduri, D., Stock, A., Marron, T., Xie, H., Patel, M. K., Oekelen, O. van, Rahman, A., Kovatch, P., Aberg, J., Schadt, E., Jagannath, S., ... Gnjatic, S. (2020). An inflammatory cytokine signature helps predict COVID-19 severity and death. medRxiv, 2020.05 .28 .20115758$. https://doi.org/10.1101/2020.05.28.20115758

Vanegas-Cedillo, P. E., Bello-Chavolla, O. Y., Ramírez-Pedraza, N., Rodríguez Encinas, B., Pérez Carrión, C. I., Jasso Ávila, M. I., Valladares García, J. C., Hernández-Juárez, D., Vargas-Vázquez, A., Antonio-Villa, N. E., Chapa-Ibarguengoitia, M., Leon, A. P. de, Sifuentes-Osornio, J., Aguilar-Salinas, C. A., \& Mehta, R. (2021). Serum Vitamin D levels are associated with increased COVID-19 severity and mortality independent of visceral adiposity [Preprint]. Endocrinology (including Diabetes Mellitus; Metabolic Disease).https://doi.org/10.1101/2021.03.12.21253490

Vaquero, L. M., Barrado, M. E. S., Escobar, D., Arribas, P., Gonzalez, J. R., Bermejo, J. F., Doncel, C., Bastida, J. M., Hernandez, A., Jambrina, C., \& Sanchez, M. V. (2020). C-Reactive protein and SOFA score as early predictors of critical care requirement in patients with COVID-19 pneumonia in Spain. medRxiv, 2020.05.22.20110429. https://doi.org/10.1101/2020.05.22.20110429

Vardavas, C. I., \& Nikitara, K. (2020). COVID-19 and smoking: A systematic review of the evidence.Tob Induc Dis, 18, 20. https://doi.org/10.18332/tid/119324

Velasco-Rodríguez, D., Alonso-Dominguez, J.-M., Vidal Laso, R., Lainez-González, D., García-Raso, A., Martín-Herrero, S., Herrero, A., Martínez Alfonzo, I., Serrano-López, J., Jiménez-Barral, E., Nistal, S., Pérez Márquez, M., Askari, E., Castillo Álvarez, J., Núñez, A., Jiménez Rodríguez, Á., Heili-Frades, S., Pérez-Calvo, C., Górgolas, M., ... Llamas-Sillero, P. (2021). Development and validation of a predictive model of in-hospital mortality in COVID-19 patients. PLOS ONE, 16(3), e0247676. https://doi.org/10.1371/journal.pone.0247676

Veras, F. P., Pontelli, M., Silva, C., Toller-Kawahisa, J., Lima, M. de, Nascimento, D., Schneider, A., Caetite, D., Rosales, R., Colon, D., Martins, R., Castro, I., Almeida, G., Lopes, M. I., Benatti, M., 
Bonjorno, L., Giannini, M., Luppino-Assad, R., Almeida, S., ... Cunha, F. (2020). SARS-CoV-2 triggered neutrophil extracellular traps (NETs) mediate COVID-19 pathology. medRxiv, 2020.06.08.20125823. https://doi.org/10.1101/2020.06.08.20125823

Vila-Corcoles, A., Satue-Gracia, E., Vila-Rovira, A., Diego-Cabanes, C. de, Forcadell-Peris, M. J., Hospital-Guardiola, I., \& Ochoa-Gondar, O. (2020).COVID-19 TARRACO Cohort Study: Development of a predictive prognostic rule for early assessment of COVID-19 patients in primary care settings [Preprint]. Infectious Diseases (except HIV/AIDS). https://doi.org/10.1101/2020.12.11.20247932

Vila-Corcoles, A., Satue-Gracia, E., Vila-Rovira, A., Diego-Cabanes, C. de, Forcadell-Peris, M. J., \& Ochoa-Gondar, O. (2021).COVID19-related and all-cause mortality among middle-aged and older adults across the first epidemic wave of SARS-COV-2 infection in the region of Tarragona, Spain: Results from the COVID19 TARRACO Cohort Study, March-June 2020. https://doi.org/10.1101/2021.02.02.21251028

Vila-Córcoles, A., Ochoa-Gondar, O., Satué-Gracia, E. M., Torrente-Fraga, C., Gomez-Bertomeu, F., Vila-Rovira, A., Hospital-Guardiola, I., Diego-Cabanes, C. de, Bejarano-Romero, F., \& BasoraGallisà, J. (2020). Influence of prior comorbidities and chronic medications use on the risk of COVID-19 in adults: A population-based cohort study in Tarragona, Spain. BMJ Open, 10(12), e041577. https://doi.org/10.1136/bmjopen-2020-041577

Villar-Garcia, J., Vivanco-Hidalgo, R. M., Cleries, M., Martinez, E., Monterde, D., Perez-Sust, P., Garcia-Eroles, L., Sais, C., Moharra, M., \& Vela, E. (2020). Risk factors for SARS-CoV-2 infection, hospitalisation, and death in Catalonia, Spain: A population-based cross-sectional study. medRxiv, 2020.08.26.20182303. https://doi.org/10.1101/2020.08.26.20182303

Voruz, P., Allali, G., Benzakour, L., Nuber-Champier, A., Thomasson, M., Jacot, I., Pierce, J., Lalive, P., Lövblad, K.-O., Braillard, O., Coen, M., Serratrice, J., Pugin, J., Ptak, R., Guessous, I., Landis, B. N., Assal, F., \& Péron, J. A. (2021). Long COVID neuropsychological deficits after severe, moderate or mild infection[Preprint]. Neurology. https://doi.org/10.1101/2021.02.24.21252329

Wagner, R., Peterhoff, D., Beileke, S., Günther, F., Berr, M., Einhauser, S., Schütz, A., Niller, H. H., Steininger, P., Knöll, A., Tenbusch, M., Maier, C., Korn, K., Stark, K. J., Gessner, A., Burkhardt, R., Kabesch, M., Schedl, H., Küchenhoff, H., ... Überla, K. (2021). Estimates and Determinants of SARS-Cov-2 Seroprevalence and Infection Fatality Ratio Using Latent Class Analysis: The Population-Based Tirschenreuth Study in the Hardest-Hit German County in Spring 2020. Viruses, 13(6), 1118. https://doi.org/10.3390/v13061118

Wallis, T. J. M., Heiden, E., Horno, J., Welham, B., Burke, H., Freeman, A., Dexter, L., Fazleen, A., Kong, A., McQuitty, C., Watson, M., Poole, S., Brendish, N. J., Clark, T. W., Wilkinson, T. M. A., Jones, M. G., \& Marshall, B. G. (2021). Risk factors for persistent abnormality on chest radiographs at 12-weeks post hospitalisation with PCR confirmed COVID-19. Respiratory Research, 22(1), 157. https://doi.org/10.1186/s12931-021-01750-8

Wan, S., Xiang, Y., Fang, W., Zheng, Y., Li, B., Hu, Y., Lang, C., Huang, D., Sun, Q., Xiong, Y., Huang, X., Lv, J., Luo, Y., Shen, L., Yang, H., Huang, G., \& Yang, R. (2020). Clinical features and treatment of COVID-19 patients in northeast Chongqing. Journal of Medical Virology, 92(7), 797-806. https://doi.org/10.1002/jmv.25783

Wan, Y. I., Apea, V. J., Dhairyawan, R., Puthucheary, Z. A., Pearse, R. M., Orkin, C. M., \& Prowle, J. R. (2021)Ethnic disparities in hospitalisation and hospital-outcomes during the second wave of COVID-19 infection in east London [Preprint]. Epidemiology. https://doi.org/10.1101/2021.07.05.21260026

Wang, A.-L., Zhong, X., \& Hurd, Y. (2020). Comorbidity and Sociodemographic determinants in COVID-19 Mortality in an US Urban Healthcare SystemmedRxiv, 2020.06.11.20128926. https://doi.org/10.1101/2020.06.11.20128926

Wang, B., Oekelen, O. V., Mouhieddine, T., Valle, D. M. D., Richter, J., Cho, H. J., Richard, S., Chari, A., Gnjatic, S., Merad, M., Jagannath, S., Parekh, S., \& Madduri, D. (2020). A tertiary cente experience of multiple myeloma patients with COVID-19: Lessons learned and the path forward. medRxiv, 2020.06.04.20122846. https://doi.org/10.1101/2020.06.04.20122846

Wang, J. G., Liu, B., Percha, B., Pan, S., Goel, N., Mathews, K., Gao, C., Tandon, P., Tomlinson, M., Yoo, E., Howell, D., Eisenberg, E., Naymagon, L., Tremblay, D., Chokshi, K., Dua, S., Dunn, A., Powell, C., \& Bose, S. (2020). Cardiovascular disease and severe hypoxemia associated with higher rates of non-invasive respiratory support failure in COVID-19 https://doi.org/10.1101/2020.09.27.20202747

Wang, J., Su, Y., Wang, Q., Cao, Y., Wang, A., Ding, R., \& Xie, W. (2021). Sex differences in clinical characteristics and risk factors for disease severity of hospitalized patients with COVID-19. MedComm, 2(2), 247-255. https://doi.org/10.1002/mco2.66

Wang, Q., Codd, V., Raisi-Estabragh, Z., Musicha, C., Bountziouka, V., Kaptoge, S., Allara, E., Angelantonio, E. D., Butterworth, A. S., Wood, A. M., Thompson, J. R., Petersen, S. E., Harvey, N. C., Danesh, J. N., Samani, N. J., \& Nelson, C. P. (2021). Older biological age is associated with adverse COVID-19 outcomes: A cohort study in UK BiobanklPreprint]. Infectious Diseases (except HIV/AIDS). https://doi.org/10.1101/2021.03.20.21254010

Wang, R., Pan, M., Zhang, X., Han, M., Fan, X., Zhao, F., Miao, M., Xu, J., Guan, M., Deng, X., Chen, X., \& Shen, L. (2020). Epidemiological and clinical features of 125 Hospitalized Patients with COVID-19 in Fuyang, Anhui, China. International Journal of Infectious Diseases, 95, 421-428. https://doi.org/10.1016/.j.jiid.2020.03.070

Wang, Z.-H., Shu, C., Ran, X., Xie, C.-H., \& Zhang, L. (2020). Critically III Patients with Coronavirus Disease 2019 in a Designated ICU: Clinical Features and Predictors for MortalityRisk Management and Healthcare Policy, Volume 13, 833-845. https://doi.org/10.2147/RMHP.S263095

Wang, Z., Zheutlin, A., Kao, Y.-H., Ayers, K., Gross, S., Kovatch, P., Nirenberg, S., Charney, A., Nadkarni, G., De Freitas, J. K., O'Reilly, P., Just, A., Horowitz, C., Martin, G., Branch, A., Glicksberg, B. S., Charney, D., Reich, D., Oh, W. K., ... Li, L. (2020). Hospitalised COVID-19 patients of the Mount Sinai Health System: A retrospective observational study using the electronic medical records. BMJ Open, 10(10), e040441. https://doi.org/10.1136/bmjopen-2020-040441

Ward, H., Atchison, C. J., Whitaker, M., Ainslie, K. E. C., Elliott, J., Okell, L. C., Redd, R., Ashby, D., Donnelly, C. A., Barclay, W., Darzi, A., Cooke, G., Riley, S., \& Elliott, P. (2020). Antibody prevalence for SARS-CoV-2 in England following first peak of the pandemic: REACT2 study in 100,000 adults. medRxiv, 2020.08.12.20173690. https://doi.org/10.1101/2020.08.12.20173690

Weerahandi, H., Hochman, K. A., Simon, E., Blaum, C., Chodosh, J., Duan, E., Garry, K., Kahan, T., Karmen-Tuohy, S., Karpel, H., Mendoza, F., Prete, A. M., Quintana, L., Rutishauser, J., Martinez, L. S., Shah, K., Sharma, S., Simon, E., Stirniman, A., \& Horwitz, L. (2020). Post-discharge health status and symptoms in patients with severe COVID-19. medRxiv, 2020.08.11.20172742. https://doi.org/10.1101/2020.08.11.20172742 
Wei, W., Ortwine, J. K., Mang, N. S., Joseph, C., Hall, B. C., \& Prokesch, B. C. (2020). Limited Role for Antibiotics in COVID-19: Scarce Evidence of Bacterial CoinfectionmedRxiv, 2020.06.16.20133181. https://doi.org/10.1101/2020.06.16.20133181

Williamson, E. J., Walker, A. J., Bhaskaran, K., Bacon, S., Bates, C., Morton, C. E., Curtis, H. J., Mehrkar, A., Evans, D., Inglesby, P., Cockburn, J., McDonald, H. I., MacKenna, B., Tomlinson, L., Douglas, I. J., Rentsch, C. T., Mathur, R., Wong, A. Y. S., Grieve, R., ... Goldacre, B. (2020). Factors associated with COVID-19-related death using OpenSAFELY. Nature, 584(7821), 430-436. https://doi.org/10.1038/s41586-020-2521-4

Woolcott, O. O., \& Castilla-Bancayán, J. P. (2021a). The effect of age on the association between diabetes and mortality in adult patients with COVID-19 in MexicoScientific Reports, 11(1), 8386. https://doi.org/10.1038/s41598-021-88014-z

Woolcott, O. O., \& Castilla-Bancayán, J. P. (2021b). The effect of age on the association between diabetes and mortality in adult patients with COVID-19 in MexicoScientific Reports, $11(1), 8386$. https://doi.org/10.1038/s41598-021-88014-z

Woolford, S. J., D’Angelo, S., Curtis, E. M., Parsons, C. M., Ward, K. A., Dennison, E. M., Patel, H. P., Cooper, C., \& Harvey, N. C. (2020). COVID-19 and associations with frailty and multimorbidity: A prospective analysis of UK Biobank participants. Aging Clinical and Experimental Research, 32(9), 1897-1905. https://doi.org/10.1007/s40520-020-01653-6

Wu, M. A., Fossali, T., Pandolfi, L., Carsana, L., Ottolina, D., Frangipane, V., Rech, R., Tosoni, A., Agarossi, A., Cogliati, C., Meloni, F., Marchini, B., Nebuloni, M., Catena, E., \& Colombo, R. (2020). COVID-19: The key role of pulmonary capillary leakage. An observational cohort study. medRxiv, 2020.05.17.20104877. https://doi.org/10.1101/2020.05.17.20104877

Wu, X., Liu, X., Zhou, Y., Yu, H., Li, R., Zhan, Q., Ni, F., Fang, S., Lu, Y., Ding, X., Liu, H., Ewing, R. M., Jones, M. G., Hu, Y., Nie, H., \& Wang, Y. (2021). 3-month, 6-month, 9-month, and 12-month respiratory outcomes in patients following COVID-19-related hospitalisation: A prospective study. The Lancet Respiratory Medicine, 9(7), 747-754. https://doi.org/10.1016/S2213-2600(21)00174-0

Xie, Y., Chen, S., Wang, X., Li, B., Zhang, T., He, X., Sun, N., Wang, L., Zeng, H., \& Shen, Y. (2020). Early Diagnosis and Clinical Significance of Acute Cardiac Injury - Under the Iceberg: A Retrospective Cohort Study of 619 Non-critically III Hospitalized COVID-19 Pneumonia Patients. medRxiv, 2020.07.06.20147256. https://doi.org/10.1101/2020.07.06.20147256

Xu, H., Hou, K., Xu, H., Li, Z., Chen, H., Zhang, N., Xu, R., Fu, H., Sun, R., Wen, L., Xie, L., Liu, H., Zhang, K., Selvanayagam, J. B., Fu, C., Zhao, S., Yang, Z., Yang, M., \& Guo, Y. (2020). Acute Myocardial Injury of Patients with Coronavirus Disease 2019. medRxiv, 2020.03.05.20031591. https://doi.org/10.1101/2020.03.05.20031591

Yadaw, A. S., Li, Y., Bose, S., lyengar, R., Bunyavanich, S., \& Pandey, G. (2020). Clinical features of COVID-19 mortality: Development and validation of a clinical prediction model.The Lancet Digital Health, 2(10), e516-e525. https://doi.org/10.1016/S2589-7500(20)30217-X

Yan, H., Valdes, A. M., Vijay, A., Wang, S., Liang, L., Yang, S., Wang, H., Tan, X., Du, J., Jin, S., Huang, K., Jiang, F., Zhang, S., Zheng, N., Hu, Y., Cai, T., \& Aithal, G. P. (2020). Role of Drugs used for chronic disease management on Susceptibility and Severity of COVID-19: A Large Case-Control Study. medRxiv, 2020.04.24.20077875. https://doi.org/10.1101/2020.04.24.20077875

Yang, X., Yu, Y., Xu, J., Shu, H., Xia, J., Liu, H., Wu, Y., Zhang, L., Yu, Z., Fang, M., Yu, T., Wang, Y., Pan, S., Zou, X., Yuan, S., \& Shang, Y. (2020). Clinical course and outcomes of critically ill patients with SARS-CoV-2 pneumonia in Wuhan, China: A single-centered, retrospective, observational study. The Lancet Respiratory Medicine, 8(5), 475-481. https://doi.org/10.1016/S22132600(20)30079-5

Yanover, C., Mizrahi, B., Kalkstein, N., Marcus, K., Akiva, P., Barer, Y., Shalev, V., \& Chodick, G. (2020). What factors increase the risk of complications in SARS-CoV-2 positive patients? A cohort study in a nationwide Israeli health organization. medRxiv, 2020.05.07.20091652. https://doi.org/10.1101/2020.05.07.20091652

Yao, K., Hasegawa, S., Tagashira, Y., Takamatsu, A., Uenoyama, Y., Shimizu, K., Honda, H., \& Tokyo Metropolitan Tama Medical Center COVID-19 treatment team. (2021). Experience of 101 patients with coronavirus infectious disease 2019 (COVID-19) at a tertiary care center in Japan. J Infect Chemother, 27, 413-417. https://doi.org/10.1016/i.jiac.2020.11.024

Yao, Q., Wang, P., Wang, X., Qie, G., \& Chu, Y. (n.d.).A retrospective study of risk factors for severe acute respiratory syndrome coronavirus 2 infections in hospitalized adult patients https://doi.org/10.20452/pamw.15312

Yin, R., Yang, Z., Wei, Y., Li, Y., Chen, H., Liu, Z., Zhao, B., Ma, D., Dan, M., Zhang, Y., Liu, X., Leng, H., \& Xiang, D. (2020). Clinical characteristics of 106 patients with neurological diseases and co-morbid coronavirus disease 2019: A retrospective study. medRxiv, 2020.04.29.20085415. https://doi.org/10.1101/2020.04.29.20085415

Yoo, E., Percha, B., Tomlinson, M., Razuk, V., Pan, S., Basist, M., Tandon, P., Wang, J. G., Gao, C., Bose, S., \& Gidwani, U. K. (2020). Development and calibration of a simple mortality risk score for hospitalized COVID-19 adults. medRxiv, 2020.08.31.20185363. https://doi.org/10.1101/2020.08.31.20185363

Yozgat, A., Kasapoğlu, B., Can, G., Tanoğlu, A., Sakin, Y. S., Yalçin, K. S., Gürler, M., Kaplan, M., Kaban, M. G., Kirsoy, M., Kara, U., \& Kekilli, M. (2021). Long-Term Proton Pump Inhibitor Use is a Risk Factor for Mortality in Patients Hospitalized for COVID-19. Turkish Journal of Medical Sciences https://doi.org/10.3906/sag-2103-80

Yu, T., Cai, S., Zheng, Z., Cai, X., Liu, Y., Yin, S., Peng, J., \& Xu, X. (2020). Association Between Clinical Manifestations and Prognosis in Patients with COVID-19.Clinical Therapeutics, 42(6), 964972. https://doi.org/10.1016/i.clinthera.2020.04.009

Zacharioudakis, I. M., Prasad, P. J., Zervou, F. N., Basu, A., Inglima, K., Weisenberg, S. A., \& Aguero-Rosenfeld, M. E. (2020). Association of SARS-CoV-2 Genomic Load with COVID-19 Patient Outcomes. medRxiv, 2020.07.02.20145151. https://doi.org/10.1101/2020.07.02.20145151

Zell, J., Klein, J., Lucas, C., Slade, M., Liu, J., Iwasaki, A., Wisnewski, A. V., \& Redlich, C. A. (2021)Associations of SARS-CoV-2 serum IgG with occupation and demographics of military personnel [Preprint]. Occupational; Environmental Health. https://doi.org/10.1101/2021.04.21.21255881

Zeng, H., Zhang, T., He, X., Du, Y., Tong, Y., Zhang, W., \& Shen, Y. (2020). Impact of Chronic Comorbidities on Progression and Prognosis in Patients with CoVID-19: A Retrospective Cohort Study in 1031 Hospitalized Cases in Wuhan, China. medRxiv, 2020.06.14.20125997. https://doi.org/10.1101/2020.06.14.20125997

Zhan, T., Liu, M., Tang, Y., Han, Z., Cheng, X., Deng, J., Chen, X., Tian, X., \& Huang, X. (2020). Retrospective analysis of clinical characteristics of 405 patients with COVID-19.Journal of International Medical Research, 48(8), 030006052094903. https://doi.org/10.1177/0300060520949039 
Zhan, Z., Yang, X., Du, H., Zhang, C., Song, Y., Ran, X., Zhang, A., \& Yang, M. (2020). Early Improvement of Acute Respiratory Distress Syndrome in Patients with COVID-19: Insights from the Data of ICU Patients in Chongqing, China. medRxiv, 2020.07.15.20154047. https://doi.org/10.1101/2020.07.15.20154047

Zhang, Jin-jin, Cao, Y., Tan, G., Dong, X., Wang, B., Lin, J., Yan, Y., Liu, G., Akdis, M., Akdis, C. A., \& Gao, Y. (2020). Clinical, radiological, and laboratory characteristics and risk factors for severity and mortality of 289 hospitalized COVID-19 patients. Allergy, all.14496. https://doi.org/10.1111/all.14496

Zhang, Jin-jin, Dong, X., Cao, Y., Yuan, Y., Yang, Y., Yan, Y., Akdis, C. A., \& Gao, Y. (2020). Clinical characteristics of 140 patients infected with SARS-CoV-2 in Wuhan, ChinaAllergy, 75(7), 17301741. https://doi.org/10.1111/all.14238

Zhang, Q., Wang, Z., Lv, Y., Zhao, J., Dang, Q., Xu, D., Zhao, D., Liu, H., Wang, Z., Zhao, X., Xu, Z., \& Zhang, X. (2021). Clinical features and prognostic factors of patients with COVID-19 in Henan Province, China. Hum Cell, NA. https://doi.org/10.1007/s13577-021-00499-y

Zhang, Xiaoli, Cai, H., Hu, J., Lian, J., Gu, J., Zhang, S., Ye, C., Lu, Y., Jin, C., Yu, G., Jia, H., Zhang, Y., Sheng, J., Li, L., \& Yang, Y. (2020). Epidemiological, clinical characteristics of cases of SARS-CoV-2 infection with abnormal imaging findings. International Journal of Infectious Diseases, 94, 81-87. https://doi.org/10.1016/j.jijid.2020.03.040

Zhang, Xiaomeng, Li, X., Sun, Z., He, Y., Xu, W., Campbell, H., Dunlop, M. G., Timofeeva, M., \& Theodoratou, E. (2020). Physical activity and COVID-19: An observational and Mendelian randomisation study. Journal of Global Health, 10(2), 020514. https://doi.org/10.7189/jogh-10-020514

Zhang, Y., Yang, H., Li, S., Li, W.-D., Wang, J., \& Wang, Y. (2021). Association analysis framework of genetic and exposure risks for COVID-19 in middle-aged and elderly adultsMech Ageing Dev, 194. https://doi.org/10.1016/j.mad.2021.111433

Zhao, Z., Chen, A., Hou, W., Graham, J. M., Li, H., Richman, P. S., Thode, H. C., Singer, A. J., \& Duong, T. Q. (2020). Prediction model and risk scores of ICU admission and mortality in COVID-19. PLOS ONE, 15(7), e0236618. https://doi.org/10.1371/journal.pone.0236618

Zheng, K. I., Gao, F., Wang, X.-B., Sun, Q.-F., Pan, K.-H., Wang, T.-Y., Ma, H.-L., Chen, Y.-P., Liu, W.-Y., George, J., \& Zheng, M.-H. (2020). Letter to the Editor: Obesity as a risk factor for greater severity of COVID-19 in patients with metabolic associated fatty liver disease. Metabolism, 108, 154244. https://doi.org/10.1016/j.metabol.2020.154244

Zheng, Yamei, Gao, Y., Wu, B., Huang, L., Chen, Y., \& Cai, X. (2021). Characteristics and outcomes of patients with COVID-19 in Hainan, South ChinaMedicine, 100(11), e24771. https://doi.org/10.1097/MD.0000000000024771

Zheng, Yi, Xiong, C., Liu, Y., Qian, X., Tang, Y., Liu, L., Leung, E. L.-H., \& Wang, M. (2020). Epidemiological and clinical characteristics analysis of COVID-19 in the surrounding areas of Wuhan, Hubei Province in 2020. Pharmacological Research, 157, 104821. https://doi.org/10.1016/j.phrs.2020.104821

Zhong, R., Chen, L., Zhang, Q., Li, B., Qiu, Y., Wang, W., Tan, D., \& Zou, Y. (2020). Which Factors, Smoking, Drinking Alcohol, Betel Quid Chewing, or Underlying Diseases, Are More Likely to Influence the Severity of COVID-19? Front Physiol, 11. https://doi.org/10.3389/fphys.2020.623498

Zhou, F., Yu, T., Du, R., Fan, G., Liu, Y., Liu, Z., Xiang, J., Wang, Y., Song, B., Gu, X., Guan, L., Wei, Y., Li, H., Wu, X., Xu, J., Tu, S., Zhang, Y., Chen, H., \& Cao, B. (2020). Clinical course and risk factors for mortality of adult inpatients with COVID-19 in Wuhan, China: A retrospective cohort study. The Lancet, 395(10229), 1054-1062. https://doi.org/10.1016/S0140-6736(20)30566-3

Zhou, J., Ma, Y., Liu, Y., Xiang, Y., Tao, C., Yu, H., \& Huang, J. (2021). A Correlation Analysis between the Nutritional Status and Prognosis of COVID-19 PatientsJ Nutr Health Aging, 25, 84-93. https://doi.org/10.1007/s12603-020-1457-6

Zhou, K., Sun, Y., Li, L., Zang, Z., Wang, J., Li, J., Liang, J., Zhang, F., Zhang, Q., Ge, W., Chen, H., Sun, X., Yue, L., Wu, X., Shen, B., Xu, J., Zhu, H., Chen, S., Yang, H., ... Guo, T. (2020). Eleven Routine Clinical Features Predict COVID-19 Severity. medRxiv, 2020.07.28.20163022. https://doi.org/10.1101/2020.07.28.20163022

Zhou, Wei, Qin, X., Hu, X., Lu, Y., \& Pan, J. (2020). Prognosis models for severe and critical COVID-19 based on the Charlson and Elixhauser comorbidity indices International Journal of Medical Sciences, 17(15), 2257-2263. https://doi.org/10.7150/ijms.50007

Zhou, Wenqian, Song, L., Wang, X., Xu, Z., Wang, S., Wang, J., Xu, H., Zheng, Y., \& Wang, Y. (2020). Cardiac injury prediction and lymphocyte immunity and inflammation analysis in hospitalized patients with coronavirus disease 2019 (COVID-19). International Journal of Cardiology, S016752732034002X. https://doi.org/10.1016/j.jicard.2020.10.049

Zhou, Yaya, He, X., Zhang, J., Xue, Y. e., Liang, M., Yang, B., Ma, W., Zhou, Q., Chen, L., \& Wang, X. (2020). Prolonged SARS-CoV-2 Viral Shedding in Patients with COVID-19 was Associated with Delayed Initiation of Arbidol Treatment: A retrospective cohort study. medRxiv, 2020.06.09.20076646. https://doi.org/10.1101/2020.06.09.20076646

Zhou, Yiwu, He, Y., Yang, H., Yu, H., Wang, T., Chen, Z., Yao, R., \& Liang, Z. (2020). Exploiting an early warning Nomogram for predicting the risk of ICU admission in patients with COVID-19: A multi-center study in China. Scandinavian Journal of Trauma, Resuscitation and Emergency Medicine, 28(1), 106. https://doi.org/10.1186/s13049-020-00795-w

Zhu, S., Gao, Q., Yang, L., Yang, Y., Xia, W., Cai, X., Hui, Y., Zhu, D., Zhang, Y., Zhang, G., Wu, S., Wang, Y., Zhou, Z., Liu, H., Zhang, C., Zhang, B., Yang, J., Feng, M., Ni, Z., ... Reinhardt, J. D. (2020). Prevalence and risk factors of disability and anxiety in a retrospective cohort of 432 survivors of Coronavirus Disease-2019 (Covid-19) from China. PloS One, 15. https://doi.org/10.1371/journal.pone.0243883

Ziehr, D. R., Alladina, J., Petri, C. R., Maley, J. H., Moskowitz, A., Medoff, B. D., Hibbert, K. A., Thompson, B. T., \& Hardin, C. C. (2020). Respiratory Pathophysiology of Mechanically Ventilated Patients with COVID-19: A Cohort Study. American Journal of Respiratory and Critical Care Medicine, 201(12), 1560-1564. https://doi.org/10.1164/rccm.202004-1163LE

Zinellu, A., Arru, F., De Vito, A., Sassu, A., Valdes, G., Scano, V., Zinellu, E., Perra, R., Madeddu, G., Carru, C., Pirina, P., Mangoni, A. A., Babudieri, S., \& Fois, A. G. (2021). The De Ritis ratio as prognostic biomarker of in-hospital mortality in COVID-19 patients. European Journal of Clinical Investigation, 51(1). https://doi.org/10.1111/eci.13427

Zobairy, H., Shamsoddin, E., Rasouli, M. A., Khodlan, N. V., Moradi, G., Zareie, B., Teymori, S., Asadi, J., Sofi-Mahmudi, A., \& Sedaghat, A. R. (2020). Association of olfactory dysfunction with hospitalization for COVID-19: A multicenter study in Kurdistan. medRxiv, 2020.07.26.20158550. https://doi.org/10.1101/2020.07.26.20158550

Zuo, Y., Estes, S. K., Gandhi, A. A., Yalavarthi, S., Ali, R. A., Shi, H., Sule, G., Gockman, K., Madison, J. A., Zuo, M., Woodard, W., Lezak, S. P., Lugogo, N. L., Kanthi, Y., \& Knight, J. S. (2020), 
Prothrombotic antiphospholipid antibodies in COVID-19. medRxiv, 2020.06.15.20131607. https://doi.org/10.1101/2020.06.15.20131607

Zuo, Y., Warnock, M., Harbaugh, A., Yalavarthi, S., Gockman, K., Zuo, M., Madison, J. A., Knight, J. S., Kanthi, Y., \& Lawrence, D. A. (2020).Plasma tissue plasminogen activator and plasminogen activator inhibitor-1 in hospitalized COVID-19 patients [Preprint]. Infectious Diseases (except HIV/AIDS).https://doi.org/10.1101/2020.08.29.20184358

Zuo, Y., Yalavarthi, S., Shi, H., Gockman, K., Zuo, M., Madison, J. A., Blair, C. N., Weber, A., Barnes, B. J., Egeblad, M., Woods, R. J., Kanthi, Y., \& Knight, J. S. (2020). Neutrophil extracellular traps in COVID-19. JCI Insight. https://doi.org/10.1172/jci.insight.138999 